\title{
Sensing with colors
}

$\Delta$
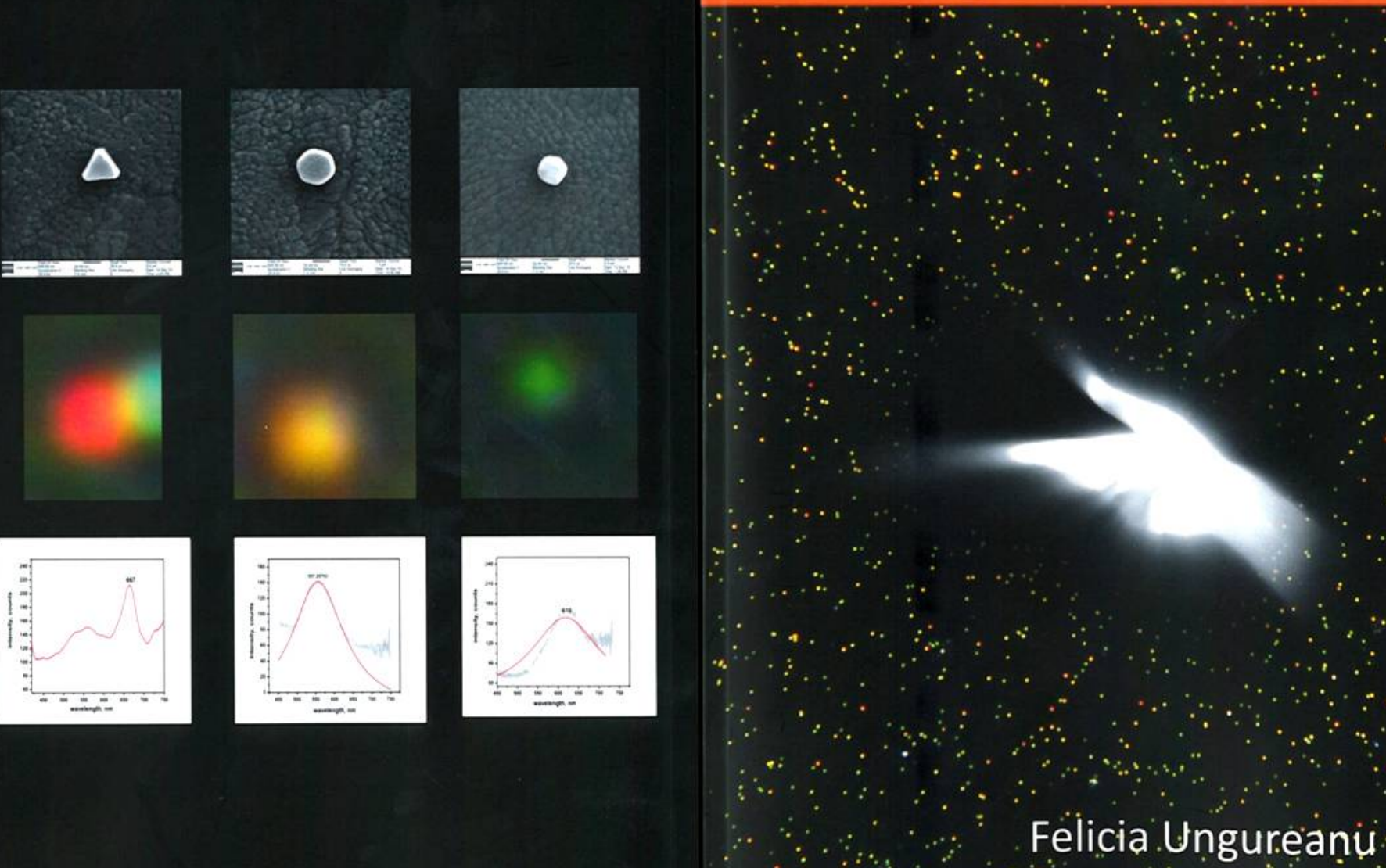

Fêlicia Ungureanụ 


\section{Sensing with colors}

by

Felicia Ungureanu 


\section{Members of the dissertation committee:}

Prof. Dr. G. van der Steenhoven University of Twente (chairman)

Prof. Dr. V. Subramaniam University of Twente (thesis advisor)

Dr. R.P.H. Kooyman University of Twente (assistant-advisor)

Dr. E.S. Kooij University of Twente, The Netherlands

Prof. Dr. J. Eijkel University of Twente, The Netherlands

Prof.Dr. J. Herek University of Twente, The Netherlands

Prof. Dr. I. T. Young University of Delft, The Netherlands

The work described in this thesis was performed at the Nanobiophysics (NBP) group, MIRA Institute for biomedical technology and technical medicine, Faculty of Science and Technology, University of Twente, PO Box 217, 7500 AE Enschede, The Netherlands.

This research has been financially supported Microned SMACT 2F workpackage

Cover Design: Felicia Ungureanu

Printed by: Wöhrmann Print Service B.V., Zutphen, The Netherlands.

ISBN : 978-90-365-3398-0

DOI nummer : $10.3990 / 1.9789036533980$

Officiële URL: http://dx.doi.org/10.3990/1.9789036533980

E-mail: fungureanu@telfort.nl

Copyright (C) Felicia Ungureanu, 2012

All rights reserved. No part of the material protected by this copyright notice may be reproduced or utilized in any form or by any means, electronic or mechanical, including photo copying, recording or by any future information storage and retrieval system, without prior permission from the author. 


\title{
Sensing with colors
}

\author{
DISSERTATION
}

to obtain

the degree of doctor at the University of Twente, on the authority of the rector magnificus, Prof.dr. H. Brinksma, on account of the decision of the graduation committee, to be publicly defended on Thursday the $6^{\text {th }}$ of September 2012 at 16:45

by

\section{Felicia Ungureanu}

Born on the $16^{\text {th }}$ of July, 1980

In Sulina, Romania 
This dissertation has been approved by:

Promotor: Prof. Dr. V. Subramaniam

Assistant promotor: Dr. R.P.H. Kooyman 
To my dearest:

Gabriel \& Ana \& Cosmin 



\section{Table of Content:}

Table of Content: $\quad$ i

Table of figures: $\quad v$

Acronym List 1

$\begin{array}{ll}\text { Chapter } 1 & 5\end{array}$

Strategies for ultra-sensitive detection of biomolecules: A review

1. Introduction 6

2. Optical detection at surfaces 9

2.1. SPR based biosensors 9

2.2. Waveguide biosensors 11

2.2.1 Resonance Mirror 11

2.2.2 Interferometric biosensors 12

$\begin{array}{ll}2.2 .3 \text { Fiberoptics biosensors } & 14\end{array}$

3. Magnotech 15

4. Nanoparticle based sensing strategies 17

4.1. Aggregation-based biosensing strategies 18

4.2. Biosensors based on the LSPR monitoring of individual nanoparticles 20

5. Conclusions 23

6. Outline of the thesis 23

$\begin{array}{ll}\text { 7. References } & 25\end{array}$

$\begin{array}{ll}\text { Chapter } 2 & 35\end{array}$

Au nanoparticles as sensing platforms

1. Introduction 36 
2. Localized Surface Plasmon Resonance 37

2.1. Quasi-static approximation

2.2. Discrete Dipole Approximation (DDA) method 40

3. Gold nanoparticles as sensing elements 41

4. Single nanoparticle sensing 43

5. Coupled nanoparticle sensing 48

5.1. Quasi-static approach 48

5.2. Numerical results 52

6. Conclusions 58

7. References 60

\section{Chapter 3}

Sensing with colors 65

1. Introduction 67

2. Experimental section 68

2.1. Detection principle 68

2.2.1 Dark field microscopy

2.2.2 Total Internal Reflection $\quad 70$

2.2. Optical setup 71

2.3. Materials 73

2.4. HIM experiments 73

$\begin{array}{ll}\text { 2.5. Data analysis } & 74\end{array}$

3. Results and discussion $\quad 78$

3.1. Colour camera as detector for spectral shifts 80

3.2. Calibration of the camera 85

3.3. Protein adsorption assay $\quad 91$

3.4. Quantification 94

3.5. Protein immunoassay 95

3.6. Concentration dependence 98 
4. Comparison between two detection methods: colour camera and two monochrome $\begin{array}{lr}\text { camera with filters } & 100\end{array}$

$\begin{array}{ll}\text { 5. Conclusions } & 105\end{array}$

$\begin{array}{ll}\text { 6. Appendix } & 106\end{array}$

$\begin{array}{ll}\text { 7. References } & 109\end{array}$

$\begin{array}{ll}\text { Chapter } 4 & 113\end{array}$

Detection of individual binding events in a direct immunoassay 113

$\begin{array}{ll}\text { 1. Introduction } & 114\end{array}$

2. Materials and methods 116

2.1. Calculations 116

2.2. Experimental section 118

2.2.1 Preparation of gold nanoparticle (AuNP) conjugates 119

$\begin{array}{ll}2.2 .2 \text { DF experiment } & 119\end{array}$

$\begin{array}{ll}2.2 .3 \text { SEM experiments } & 121\end{array}$

$\begin{array}{lr}\text { 2.2.4 DF images analysis } & 122\end{array}$

3. Results and discussions 124

3.1. DF results 124

3.2. SEM results 126

3.3. Experimental determination of the surface coverage, $\Gamma \quad 128$

$\begin{array}{ll}\text { 4. Conclusions } & 130\end{array}$

$\begin{array}{ll}\text { 5. References } & 131\end{array}$

\section{Chapter 5}

Performance of colorimetric darkfield microscopy in an amplified sandwich immunoassay 135

1. Introduction 136

2. Materials and methods 138

2.1. Assay design: choice of the nanoparticle pair 139 
2.2. Bioconjugation of gold nanoparticles

2.3. Sensor preparation

2.4. Optical detection of the immunoassay 142

2.5. SEM imaging 144

2.6. Data analysis 145

3. Results 146

3.1. Validation of the activity of the conjugated nanoparticles 146

3.2. DF results: detection of individual binding events in a sandwich assay 148

3.3. Statistics on the $\mathrm{r} / \mathrm{g}$ change- sample and control 151

3.4. SEM results 152

3.5. LOD of the system 154

4. Conclusions 157

5. References 160

\section{Chapter 6}

Conclusions 165

1. References: 171

Samenvatting 173

Acknowledgements 175

List of publications 177 


\section{Table of figures:}

Figure 1-1 Schematic representation of a biosensing device. The binding of a specific molecular species (analyte) from the complex matrix to the recognition layer (receptor molecules) will lead to a non-electrical signal. The transduction module transforms this signal into an electrical signal, which is processed and sent to a display.

Figure 1-2 Artistic representation of the SPR method (Kretschmann configuration). (a) The light hitting a hemispherical prism at total internal reflectance induces an evanescent field on both sides of the metal film. Binding events on the sensing layer lead to changes in the local refractive index resulting in an angle shift. (b) Reflectance curve showing a dip in the reflected light at resonance conditions. When binding events occur on the sensing layer the dip shifts accordingly. (c) A typical SPR sensogram. When analytes bind to the surface the resonance angle changes until it reaches an end value dictated by the equilibrium condition, as expressed by eq. 1-2. When complete dissociation occurs, the resonance angle shifts back towards the baseline.

Figure 1-3 Illustration of the working principle of an RM biosensor. A polarized focused beam is sent, under total internal reflection conditions, to the transducer surface. The light couples in the waveguide layer via the evanescent wave. The resonance conditions change when the local refractive index on the sensing area changes, leading to a change in the angle of incidence [33]. RM biosensors have been used for over a decade [34,35] and are available on the market[36].

Figure 1-4 Schematic representation of some interferometric biosensors: (a) Mach Zehnder Interferometer sensor [38], (b) Young Interferometer biosensor [39]

Figure 1-5 Schematic representation of several structures of fiber optics sensors. (a) FBG sensor[53]; (b) Fabry-Perot sensor [33]

Figure 1-6(a) The actuation process of the magnetic particles inside the cartridge during the immunoassay. A- The functionalized magnetic particles react with the target molecules and are carried through capillary flow towards the active area; B- A magnetic field attracts all the magnetic nanoparticles to the active zone, where only particles with bound target molecules will be locked on the surface; C- A second magnetic field, oriented in the opposite direction separates the nonbound magnetic particles from the active area. (b) The optical detection of bound magnetic particles by frustrated total internal reflectance (FTIR). Here the intensity of the reflected beam is decreased 
when magnetic particles adhere to the active area, due to scattering and absorption effects. The decrease is proportional with the amount of particles bound to the surface.

Figure 1-7 Schematic representation of an aggregation assay. (a) Functionalized particles exposed to (b) analyte molecules will lead to immuno-binding/hybridization resulting in a network of conjugated nanoparticles linked to each other by the analyte molecules. The colour of the solution changes due to aggregation of the conjugates.

Figure 1-8 Colorimetric detection of DNA hybridization. DNA functionalized nanoparticles are hybridized in solution with a DNA target molecule. The sample is then spotted on an illuminated glass slide and the scattered light is visualized [97].

Figure 1-9 Schematic representation of a nanoparticle - based sensing approach. The surface is chemically modified (a) to bind covalently/by adsorption the functionalized nanoparticles (b). The ready-to-use sensor is then introduced in a transducing system (e.g. DF microscope, TIR) and the signal (e.g. scattering spectrum, colour) of individual nanoparticles is measured. In the beginning of the assay, the nanoparticle is characterized by a certain local refractive index determined by the molecules of the surrounding medium (e.g. buffer, water). When a binding event occurs, the local refractive index around the nanoparticles changes as the analyte molecule replaces one or more molecules of the surrounding medium. As a result, the optical properties of the nanoparticle change and a shifted signal is measured.

Figure 2-1 Schematic representation of a spherical particle, of radius $\mathrm{R}$ embedded in a surrounding medium, $\varepsilon_{\mathrm{m}}$, placed in a constant electric field, $\mathrm{E}_{0}$

Figure 2-2 (a) Schematic representation of the LSPR effect, showing the displacement of the electronic cloud in respect to the positive nuclei (b) Typical scattering spectrum of an individual 80 $\mathrm{nm} \mathrm{Au}$ particle immobilized on glass. The grey curve represents the raw spectra while the fitting is represented by the red curve.

Figure 2-3 A darkfield image $\left(270 \mathrm{x} 165 \mu \mathrm{m}^{2}\right)$ of individual $80 \mathrm{~nm}$ Au nanoparticles immobilized on glass surface. The variations in color are given by the different spectral properties of the individual nanoparticles.

Figure 2-4 Schematic representation of the sensing principle using gold nanoparticle as sensing element. (a) Non-amplified assay where the target interacts with the receptor molecules immobilized on the surface of the gold nanoparticle. (b) Amplified direct assay; the target 
molecules, linked on the surface of the secondary particles react with the receptor molecules on the immobilized nanoparticle bringing the two nanoparticles in close proximity.

Figure 2-5 (a) A schematic representation of the layered model used in our simulations. The Au nanoparticle of radius $\mathrm{R}$, covered by a shell of protein of thickness $\mathrm{d}$, is embedded in water, with a refractive index, $\mathrm{n}_{\mathrm{w}}=1.33$. (b) The results of the extension of the field for various particle sizes.

Figure 2-6 The spectral response of a $60 \mathrm{~nm}$ Au particle, embedded in water, to an increasing number of adsorbed $6 \mathrm{~nm}$ diameter biomolecules.

Figure 2-7 (a) The distribution of the Au nanoparticles with a certain occupation rate for $C_{x}=$ $1.7 * 10^{-8} \mathrm{M}$. (b) The theoretical concentration dependence

Figure 2-8 Schematic representation of the behavior of two closely located particles placed in an electric field perpendicular to the system axis

Figure 2-9 Schematic representation of the particle system in respect to the polarization of light: (a) un-polarized, (b) parallel with the system axis and (c) perpendicular. The distance $d$ is defined as the surface to surface inter-particle distance. The light propagation is along the $\mathrm{X}$-axis.

Figure 2-10 The numerical result in the case of two-particle system for different polarizations. (a) The scattering spectra for the $\mathrm{Au}^{80}-\mathrm{Au}^{40}$ particle system at different inter-particle distances; (b) The expected wavelength shifts for different polarizations states of the incident light

Figure 2-11 (a) The dependence of the plasmon peak on the size of the secondary particle for a $10 \mathrm{~nm}$ (square) , $20 \mathrm{~nm}$ (circles) and 100nm inter-particle distance. (b) The theoretical plasmon peak shifts for different coupled nanoparticle systems obtained for various inter-particle distances. The obtained results are for a particle pair in un-polarized light.

Figure 2-12 (a) An illustration representing the position of the added particles in respect to the 80 $\mathrm{nm}$ Au particle (b) The expected wavelength shift range when the position of the secondary particle is varied.

Figure 2-13 (a) Drawing of the simulated system in un-polarized light. (b) The expected spectral shift in an $\mathrm{Au}^{80}-\mathrm{Au}^{40}$ system at $20 \mathrm{~nm}$ apart when multiple binding events are expected. The system was considered in un-polarized light. The purpose of the red line is to guide the eye. 
Figure 2-14 The theoretical limits of detection for an $\mathrm{Au}^{80}-\mathrm{Au}^{40}$ pair in an assay where the affinity constant of the analyte (a) or the number of available particles (b) is changed.

Figure 3-1 a) Schematic representation of the DF principle [17] ; b) polymer grid under DF illumination.

Figure 3-2 a) The schematic representation of the TIR principle. The light travelling from the initial medium, with refractive index $\mathrm{n}_{1}\left(\mathrm{n}_{1}>\mathrm{n}_{2}\right)$, at a small angle in respect to the normal to the surface (red lines), will partially be refracted in medium $\mathrm{n}_{2}$ and partially reflected. When the incident angle is larger than the critical angle the light is totally reflected in the medium $n_{1}$. b) Typical image of $\mathrm{Au}$ NPs imaged using a TIR setup. The red hue is a chromatic aberration resulted from small misalignments and mismatches in the experimental setup.

Figure 3-3 The experimental setup

Figure 3-4 Screenshots of the analysis program. (a) the digital mask applied on the DF image showing the selection criteria. Here a very narrow range of particle sizes was selected (only $60 \mathrm{~nm}$ Au nanoparticles). (b)The nanoparticles selected for analysis after the digital mask was applied; (c) The selected nanoparticles, showing binding, on the DF image. The role of the false colours, which show the localization of the selected nanoparticles, is to guide the eye of the observer.

Figure 3-5 (a) A typical DF image of $60 \mathrm{~nm}$ Au particles immobilized on a glass surface (1.4 $* 10^{10}$ particles $/ \mathrm{ml}$ and incubation time 5 minutes) (b) SEM image of $60 \mathrm{~nm}$ Au particles immobilized on glass with a larger incubation time(20 minutes).

Figure 3-6 (a) The colour distribution of $60 \mathrm{~nm}$ particles from a DF image with an FOV $=230 \times 170$ $\mu \mathrm{m}^{2}$; (b)The size distribution of Au nanoparticles determined from SEM images for a total of100 nanoparticles

Figure 3-7 (a) Coloured solutions with $\mathrm{pH}$ indicators suggesting they have different $\mathrm{pH}$ 's; (b) The spectra of a number of $\mathrm{pH}$ solutions.

Figure 3-8 (a) The image of a pH solution in bright-field (BF) conditions; (b) The r/g dependence on the illumination conditions for three $\mathrm{pH}$ solutions.

Figure 3-9 (a) The absorption spectra for a series of solutions of a certain $\mathrm{pH}$; (b) The r/g dependence on the absorption level of the $\mathrm{pH}$ solution. 
Figure 3-10 The stability of the r/g value of several gold nanoparticles.

Figure 3-11 The absorption spectra of three solutions with $1 \mathrm{~nm}$ shift of the maximum

Figure 3-12 The simulated and experimental spectra of an individual (a) $60 \mathrm{~nm}$ and (b) $80 \mathrm{~nm} \mathrm{Au}$ particle in water

Figure 3-13 (a) A characteristic signal during refractive index experiment of a $60 \mathrm{~nm} \mathrm{Au}$ nanoparticle; (b) the corresponding spectral information of the same nanoparticle.

Figure 3-14 The sensitivity curves for $60 \mathrm{~nm}$ Au particle determined theoretically (circles), for a single nanoparticle immobilized on surface (triangles) and for a distribution of particles in bulk solution (squares).

Figure 3-15 The calibration curves of the colour camera for (a) $60 \mathrm{~nm}$ Au particle and (b) $80 \mathrm{~nm}$ Au particle

Figure 3-16 (a) HIM and (b) the corresponding DF image of the same nanoparticles $\left(\sim 20 \mu \mathrm{m}^{2}\right)$

Figure 3-18 (a) Correlation between the position of the PP and radius of the particles obtained from the spectroscopic and HIM data. (b) Size calibration of the colour camera

Figure 3-19 Schematic representation of the protein assay. The surface chip is incubated for 45 minutes with thiolated anti-goat antibody $(\mathrm{AgSH})$ and then washed with MQ water.

Figure 3-20 The schematic representation of the flow-cell.

Figure 3-21 Two characteristic signals of two individual nanoparticles.

Figure 3-22 Typical signals from two individual nanoparticles in the control experiment

Figure 3-23 The quantified curves of two measured binding curves

Figure 3-24 Illustration of the experiment design. The conjugated nanoparticles were immobilized on glass surface (a) and the target molecules were incubated for 120 minutes (b). Immediately afterwards, the sensor surface was washed with PBS buffer (c). 
$\overline{\text { Figure 3-25 Two signals from two individual nanoparticles showing immunobinding (sample) }}$

Figure 3-26 Two signals from individual nanoparticles with no binding event (control)

Figure 3-27 Selected nanoparticles with a colour change larger than the threshold in a (a) sample and (b) control. On average, we have 2000 analyzed particles in each FOV. We used a concentration of $800 \mathrm{nM}$ of goat $\mathrm{IgG}$ for the sample and $800 \mathrm{nM}$ of HRP

Figure 3-28 The response of multiple individual nanoparticles after the incubation with various concentrations of analyte (squares) or control (circles). The response was normalized for the comparison of the experimental data with the Monte Carlo simulation binding model.

Figure 3-29 The relative spectral response of the CCD chip (ICX285AL) when a (a) colour filter (QEmax $\sim 58 \%$ )is present or (b) absent (QEmax $\sim 65 \%$ )

Figure 3-30 the spectral response of the selected filters in the $480-600 \mathrm{~nm}$ region.

Figure 3-31 The calculated spectral response of the (a) colour camera and (b) two filters when the spectrum of a single nanoparticle is measured.

Figure 3-32 (a) The shifting of the spectral maximum of an individual gold nanoparticle from 525 to $575 \mathrm{~nm}$ in steps of $1 \mathrm{~nm}$. (b) The response of the filters and the Red and Green channel on the selected spectral range.

Figure 3-33 The noise levels of the CCD chip

Figure 3-34 (a) The response of the two detection systems to spectral shifts in the 525-575nm spectral range. (b) The minimum detectable wavelength shifts of the two detection systems (the ripple effect on the data is given by the simulated spectrum of the nanoparticle).

Figure 4-1 Plasmon resonance peak shifts of an $80 \mathrm{~nm}$ Au particle, p1, coupled to a second particle, $\mathrm{p} 2$, as a function of the diameter of p2 for $10 \mathrm{~nm}$ (squares), $20 \mathrm{~nm}$ (spheres) and $100 \mathrm{~nm}$ (triangles) inter-particle distances, $\mathrm{d}$. The inset shows a schematic drawing to determine the parameter $\mathrm{d}, \mathrm{p} 1$, $\mathrm{p} 2$, as well as the direction of the incident light, S, with regard to the coordinate system.

Figure 4-2 Schematic representation of the binding assay conducted here: rabbit anti-goat antibody functionalized $80 \mathrm{~nm}$ Au nanoparticles $\left(\alpha g o a t-\mathrm{Au}^{80}\right.$ ) immobilized on a BSA coated glass substrate 
binding goat IgG functionalized $40 \mathrm{~nm} \mathrm{Au}$ nanoparticles (goat $\mathrm{IgG}-\mathrm{Au}^{40}$ ) during incubation from solution. In the reference experiment goat $\operatorname{IgG}$ has been replaced by non-binding horseradish peroxidase (HRP).

Figure 4-3 (a) Typical darkfield image of immobilized $80 \mathrm{~nm}$ diameter AuNP-conjugates of a specific field of view (FOV). (b) Some particles that show evidence of color change: left column shows the particles at the beginning while right column shows the particles at the end of the experiment.

Figure 4-4 a) The wavelength and refractive index calibration of the colour camera on a individual $60 \mathrm{~nm}$ AuNp.b)The scattering spectra of the same particle in $n=1.33$ (black) and $n=1.42$ (red).

Figure 4-5 Time traces of $\mathrm{r} / \mathrm{g}$ of 4 individual particles from the immuno assay ( $\alpha$ goat-Au ${ }^{80}-$ goat $\left.\operatorname{Ig} G-A^{40}\right)$. The washing steps are indicated by a white background while the incubation period with goat $\mathrm{IgG}-\mathrm{Au}^{40}$ is indicated by a yellow background.

Figure 4-6 Time traces of $r / g$ of 4 individual particles from the reference $\left(\alpha g o a t-A u^{80}-H R P-A u^{40}\right)$. Washing steps are indicated by regions with white background, while incubation with $\mathrm{HRP}-\mathrm{Au}^{40}$ is indicated by a region with yellow background.

Figure 4-7 SEM images of the sample (a) and reference immunoassay (b). In both images, the 80 $\mathrm{nm}$ particles with at least one $40 \mathrm{~nm}$ particle binding to them are tagged with white circles. For two of those particles a zoom is shown as inset.

Figure 4-8 DF images of sample (left) and reference (right) after using the subtraction program, indicating only those particles that show a significant $\mathrm{r} / \mathrm{g}$ change.

Figure 4-9 The time-dependent immuno-binding of goat IgG- $\mathrm{Au}^{40}$ particles to $\alpha$ goat- $\mathrm{Au}^{80}$ particles in the DF. Integration of each binding event of all analyzed particles in time.

Figure 5-1 Artistic representation of the sandwich assay performed in this study: The 'developer' $\left(\alpha \mathrm{HSA}-\mathrm{Au}^{40}\right)$ will bind to the target molecules (HSA); this is bound to the receptor molecules $(\alpha \mathrm{HSA})$ found on the immobilized nanoparticle.

Figure 5-2 Schematic representation of the conjugation protocol of Au nanoparticles with protein molecules

Figure 5-3 The flow assay-schematic representation. For details, see text. 
Figure 5-4 DF image of $\alpha \mathrm{HSA}-\mathrm{Au}^{80}$ immobilized on glass substrate (a) after the target molecules were incubated $\left(\alpha \mathrm{HSA}-\mathrm{Au}^{80}-\mathrm{HSA}\right)$ and $(\mathrm{b})$ at the end of the immuno - assay experiment $(\alpha \mathrm{HSA}$ $\left.\mathrm{Au}^{80}-\mathrm{HSA}-\alpha \mathrm{HSA}-\mathrm{Au}^{40}\right)$. The FOV of the image is $270 \mathrm{x} 165 \mu \mathrm{m}^{2}$

Figure 5-5 The absorption spectra of the conjugates (in bulk) used in the sandwich assay.

Figure 5-6 The normalized absorption spectra of the (a) $\alpha \mathrm{HSA}-\mathrm{Au}^{80}$ and (b) $\alpha \mathrm{HSA}-\mathrm{Au}^{40}$ conjugates. A wavelength shift is expected when the partner molecule is added in solution. The control experiments show no shifts when a non-interacting protein is added into the conjugates solution.

Figure 5-7 (a) The change in the signal in time for two individual $80 \mathrm{~nm}$ Au particles (b) DF image selection depicting the particle at the beginning and at the end of the experiment

Figure 5-8 (a) The change of the signal in time of two individual nanoparticles in the control experiment. (b) The corresponding DF crops of the presented particles atthe beginning and at the end of the experiment.

Figure 5-9 Comparison between the colour change distribution between the sample and the control.

Figure 5-10 SEM images of the sample (a) and control immunoassay (b). The $80 \mathrm{~nm}$ particles with at least one $40 \mathrm{~nm}$ particle binding to them are tagged with black circles. The inset in the sample image shows a zoom of the $80 \mathrm{~nm}$ functionalized particle with a few bound $40 \mathrm{~nm}$ conjugated particles.

Figure 5-11 The images of the selected particles after using the selection algorithm in the (a) sample and (b) control experiment

Figure 5-12 The immunoreaction response as a function of the HSA concentration with the developer incubation time $(\tau)$ as a parameter.

Figure 5-13 The concentration dependence of the response based upon the fraction of Au nanoparticles that changed colour after the incubation of the developer. 


\section{Acronym List}

agoat - anti-goat antibody

agoat - $\mathrm{Au}^{40,60,80}-40,60,80 \mathrm{~nm}$ Au nanoparticles functionalized with anti-goat antibody

$\mathrm{HSA}$ - anti Human Serum Albumine antibody

$\mathrm{Ab}-$ Antibody

AOTF - Acusto - Optics Tunable Filters

AuNp - Au Nanoparticles

AFM - Atomic Force Microscopy

APTES - 3-Aminopropyltriethoxysilane

BF - Bright Field

BSA - Bovine Serum Albumine

CCD - Charge - Coupled Device

DDA- Discrete Dipole Approximation

DDSCAT - Software used for theoretical simulation of optical properties of nanoparticles using DDA method

DF- Dark Field

DNA - Deoxyribonucleic acid

EDC - N-(3-Dimethylaminopropyl)-N'-ethylcarbodiimide

ELISA - Enzime Linked ImmunoSorbent Assay

EtOH - Ethanol

FBG- Fiber Bragg Gratings 
FOV - Field Of View

FTIR- Frustrated Total Internal Reflectance

Goat $\operatorname{IgG}-\mathrm{Au}^{40}$ - goat $\operatorname{IgG}$ functionalized $40 \mathrm{~nm}$ Au nanoparticles

GNP - Gold NanoParticle

HIM - Helium Ion Microscopy

HRP - horseradish peroxidise

HSA - Human Serum Albumine

IgG - Immunoglobulin G

ITO- Indium Tin Oxidase

LCTF - Liquid Crystal Tunable Filters

LOD - Limit of Detection

LPG- Long Period Gratings

LSPR- Localized Surface Plasmon Resonance

MC - Monte Carlo

MMP - Multiple Multipole Method

MQ- MilliQ water

MUA - mercapto undecanoic acid

MZI - Mach Zehnder Interferometer

$\mathrm{N}_{2}-$ Nitrogen gas

NHS - N-hydroxysuccinimide

ODN - oligodeoxynucleotides

PBS - phosphate buffered saline

PSF- Point Spread Function

PP- Plasmon Peak

RGB - Red Green Blue 
RG - Red Green

R/G - Red over Green ratio

RM- Resonance Mirror

RPM - Rotations Per Minute

SEM - Scanning Electron Microscopy

SNR - Signal To Noise Ratio

SPR- Surface Plasmon Resonance

TEM - Transmission Electron Microscopy

TIR- Total Internal Reflectance

UV-VIS - UltraViolet - Visible regime

YI- Young Interferometer 


\section{Chapter 1}

\section{Strategies for ultra-sensitive detection of biomolecules: A review}

Abstract: This chapter reviews the state of the art in the development of strategies for ultra-sensitive detection of biomolecules with a focus on optical detection technologies. The principles of various sensing platforms will be described. The performances of these platforms will be evaluated and compared in terms of sensitivity and limits of detection. An overview of the work described in this thesis will be given. 


\section{Introduction}

The development of sensing platforms able to detect the presence of low levels of chemical or biological species is essential for fields ranging from medical diagnostics, safety and food industry to environmental protection.

As early as in 1957, Leland C. Clark launched the concept of the biosensor [1]. He developed an "enzyme electrode" to measure the glucose concentration using the glucose oxidase enzyme. Ever since, a wide range of biosensors has emerged.

Biosensors have been defined in many different ways. An adequate definition of a biosensor is "an analytical device that incorporates a biologically active material in intimate contact with an appropriate transduction element to detect the concentration or activity of chemical species in any type of sample" [2] .

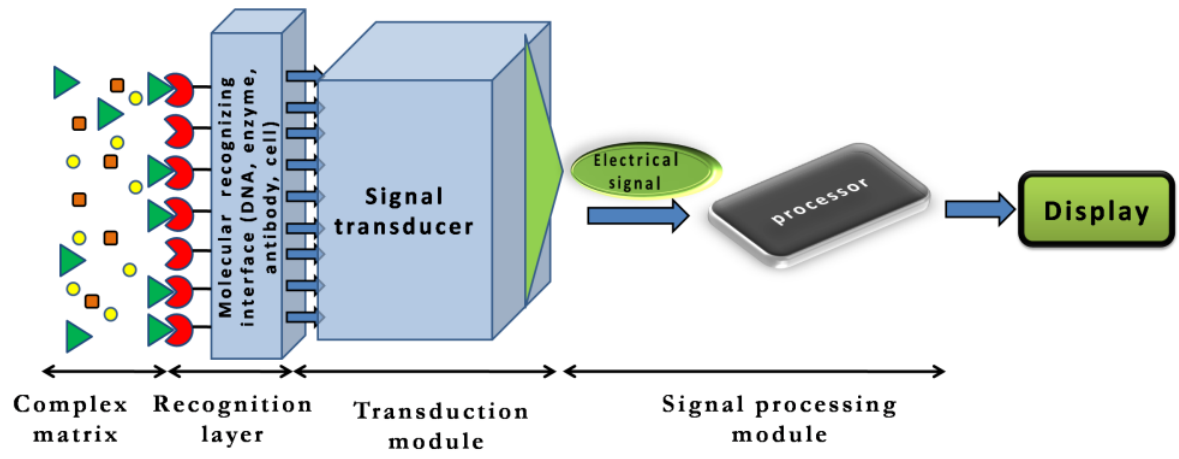

Figure 1-1 Schematic representation of a biosensing device. The binding of a specific molecular species (analyte) from the complex matrix to the recognition layer (receptor molecules) will lead to a non-electrical signal. The transduction module transforms this signal into an electrical signal, which is processed and sent to a display.

The general format of a biosensor is illustrated in Figure 1-1. An important part of the biosensor is the recognition layer; its role is to convert the biological reaction into a physico-chemical response, such as, e.g., a color change, or a change in electrical conductivity. The elementary constituents of this layer are the receptor molecules that react to specific molecules (analyte). The most important characteristics of these receptor molecules are their affinity and specificity towards the analyte. Receptor molecules can be 
$\overline{\text { all kinds of biological molecules (e.g., hormones, enzymes, DNA }{ }^{1} \text { ). An important class of }}$ receptors is antibodies: their advantage is that they can be generated against almost any molecule with a molecular mass $>\sim 400$ Dalton. In addition, they are highly specific and they bind strongly to the analyte. Their major disadvantages are their poor long-term stability and the costs associated with their development and production.

The transducer module has the role of converting the non-electrical (physicochemical) signal generated by the receptor layer into an electrical signal. Depending on the converted physical parameter, the transducer can be thermoelectric[3], piezo-electric[4-6], electro-chemical [7-9], or optical. In this chapter, we will focus our discussion on optical biosensors.

A useful classification of optical biosensors is to divide them based on the presence of a tagging agent. Hence, we have label-free and amplified methods. In labelfree methods, a direct measurement of the biological interaction is carried out. Here, the biological interaction causes a physical change (e.g. changes in electrical or optical properties) in the transducer. The big advantage of label-free methods is that they directly measure an intrinsic parameter associated with the binding process. The downside of these methods can be their low sensitivity. In amplified methods, labels are used to enhance the signal, improving the overall sensitivity. Typical labels used are fluorescent labels [10], enzymes [11, 12], and radioactive labels [13]. Apart from the fact that an additional step has to be implemented, the main disadvantages of these labels are photobleaching, quenching, and/or limited radiative time. New materials without these disadvantages are gaining popularity: quantum dots [14-16] and metal nanoparticles [1719].

Key requirements in the development of a sensor are:

(1) high specificity towards the analyte (the molecule of interest);

(2) the ability to detect very low concentrations;

\footnotetext{
${ }^{1}$ Abbreviations are explained in the Acronym List on page 1 of this thesis ${ }^{2}$ Note that, generally $\varepsilon$ and $\mathrm{n}$ are related by $\varepsilon=\mathrm{n}^{2}$

3Part of this chapter was published as "The use of a colour camera for quantitative detection of protein binding nanoparticles", Ungureanu, F., Halamek, J., Verdoold, R., Kooyman, R.P.H., Proc. SPIE 7192,
} 
(3) robust and compact;

(4) low sample volumes;

(5) fast response to enable real-time analysis;

(6) low cost.

In the development stage of a sensing methodology, a factor to be considered is the need for and complexity of pre-processing of the sample. By making possible the use of raw samples or reducing the pre-processing step, the cost-effectiveness of the sensing strategy can be increased.

The performance of a sensor system can be evaluated using the following parameters: the sensitivity (S) and limit of detection (LOD). The sensitivity can be translated as a measure of the magnitude of the sensor's response due to the receptoranalyte (e.g. antibody-antigen) binding process [20]. In this thesis, we refer to the limit of detection as the minimum detectable concentration. Generally, the magnitude of the sensor's response is determined by two separate factors:

- $\quad$ the efficiency of the chemical sensing step, denoted by $S_{\text {chem. This factor is a }}$ combination between the affinity of the receptor towards the analyte, receptor density in the receptor layer, and the resulting change in physical properties (e.g. optical, thermal, mechanical) of the receptor layer as a result of binding,

- the efficiency of transducing the changed physical property into a measurable signal, denoted by $\mathrm{S}_{\text {trans. }}$.

In the following, we will limit our discussion to the situation where antibodies form the receptor layer. The immunoreaction between the antigen $A$ and its corresponding antibody is an affinity reaction. The binding of molecule $\mathrm{A}$ is described by the relation:

$$
A b+A \stackrel{K_{a}}{\Leftrightarrow} A b A
$$

where $K_{a}$ is the affinity constant describing the affinity of the antibody $A b$ towards molecule A. Typical values of $K_{a}$ for immunoreactions range between $10^{6}-10^{11} \mathrm{M}^{-1}$. 
$\overline{\text { Ideally, an antibody should show no affinity towards other molecules than molecule A, }}$ thus $K_{x}=0\left(K_{x^{-}}\right.$the affinity constant of the antibody $A b$ towards molecule $\left.X\right)$. Given a concentration $[\mathrm{A}]$ of the analyte, the fraction of bound receptor molecules $\Gamma$ is given by:

$$
\Gamma=\frac{K_{a}[A]}{1+K_{a}[A]}
$$

One is interested to measure low concentrations of analyte molecules. Thus given a certain sensitivity of the transducer, $S_{\text {trans }}$, the limit of detection (LOD) is dictated by the number of available receptor sites and the affinity constant. The two sensitivities that define the sensor performance are interconnected: $S_{\text {chem }}$ relates the concentration of the antigen to the fraction of bound receptors, $\Gamma$, and $S_{\text {trans }}$ denotes the response of the transducer to a changing $\Gamma$.

In the following, we will focus our discussion on optical transducer technologies available for surface-based sensing systems (section 2) and on nanoparticle-based approaches (section 4).

\section{Optical detection at surfaces}

Of particular interest for surface-based optical sensor systems is the behaviour of light at an interface of two media with different refractive indices. If radiation passes such an interface, it can undergo reflection, refraction or total internal reflectance. Depending on the type of interrogation we can have, surface plasmon resonance (SPR) based biosensors, or waveguide biosensors (e.g. interferometric biosensors or fiber biosensors). Here we will discuss the optical principles of some of these platforms and present a review of their performance. All these platforms are based on the detection of a change in the refractive index at the interface due to analyte-receptor interaction.

\subsection{SPR based biosensors}

Surface Plasmon Resonance (SPR) based biosensors are one of the most successful tools used in the study of biomolecular interactions. Ever since Liedberg et al. 
[21] published the proof of principle of the method in 1983, it has undergone a rapid development and nowadays many different commercial applications exist on the market [22-24]. The principle, development and applications of SPR have been presented in many excellent reviews [25-27]. Here, we will discuss shortly the physical principles of SPR.

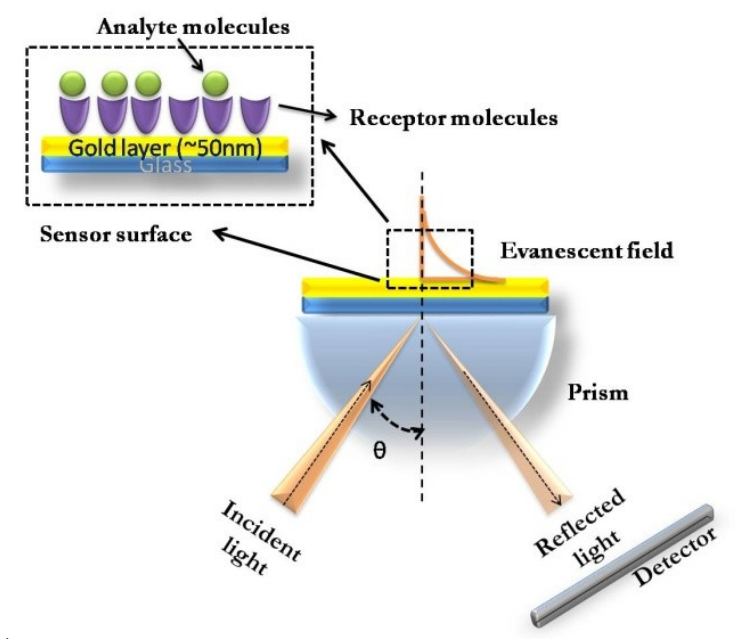

(a)

(b)

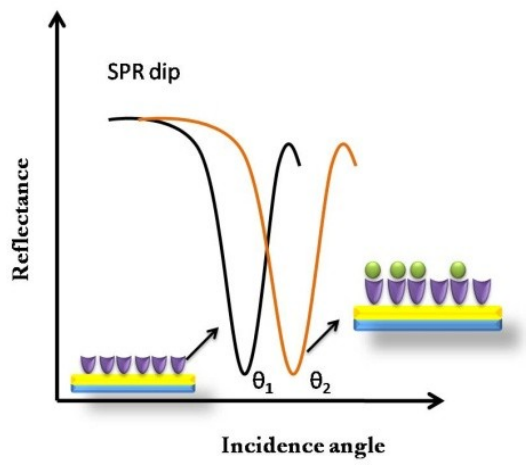

(c)

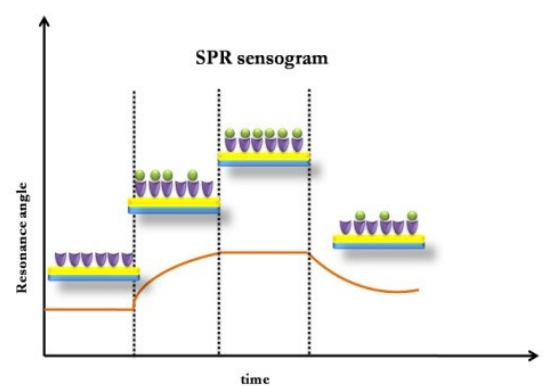

Figure 1-2 Artistic representation of the SPR method (Kretschmann configuration). (a) The light hitting a hemispherical prism at total internal reflectance induces an evanescent field on both sides of the metal film. Binding events on the sensing layer lead to changes in the local refractive index resulting in an angle shift. (b) Reflectance curve showing a dip in the reflected light at resonance conditions. When binding events occur on the sensing layer the dip shifts accordingly. (c) A typical SPR sensogram. When analytes bind to the surface the resonance angle changes until it reaches an end value dictated by the equilibrium condition, as expressed by eq. 1-2. When complete dissociation occurs, the resonance angle shifts back towards the baseline. 
The SPR effect occurs when p-polarized light, reaching the boundary of a dielectric substrate coated with a thin layer $(\sim 60 \mathrm{~nm})$ of a metal (gold or silver), is totally internally reflected. At resonance conditions, the photons hitting the metallic layer induce a resonant oscillation wave of the free electrons. As a result, an evanescent wave propagates parallel to the interface, with a penetration depth dependent on the wavelength of the incident light. At a specific angle of the incident light beam, where the resonance condition occurs, the reflected beam is strongly attenuated. This angle is dependent on the wavelength of the incoming light as well as the refractive indices of the dielectric medium and of the external medium close to the interface. Biomolecules, binding to the surface change the refractive index profile near the interface, leading to a different resonance angle. The angle shift is proportional with the amount of target bound on the surface. The detection principle is depicted in Figure 1-2. The binding of target molecules with a refractive index (e.g. proteins $(n=1.48)$, DNA $(n=1.66)$ ) higher than the refractive index of the solvent is easily detected with the SPR approach in a label-free manner. In addition, because of the confinement of the evanescent field near the surface of the sensor, the SPR signal is only moderately influenced by the refractive index of the bulk solution.

An important virtue of the SPR method is that it can easily be adapted for simultaneous detection of multiple analytes [28]. With such an extension, SPR allows in principle the parallel monitoring of thousands of biomolecular interactions.

SPR technology can detect biomolecules with a LOD as low as 10-9M (unamplified) - 10-12M (amplified)for proteins[29,30] and 10-12M (un-amplified)- 10-15M (amplified)for DNA detection [31,32].

\subsection{Waveguide biosensors}

\subsubsection{Resonance Mirror}

The Resonance Mirror (RM) technique is another interesting method used to detect changes in the local refractive index on the sensing surface.

The principle of RM is depicted in Figure 1-3. Here, a sandwich-like pattern of the transducer is made, consisting of a high index substrate prism $(n=1.72)$, a thin $(\sim 500$ $\mathrm{nm})$ low index spacer $(\mathrm{n}=1.52)$ and a very thin $(\sim 80 \mathrm{~nm})$ waveguiding layer $(\mathrm{n}=2.0)$. The 
$\overline{\text { last layer acts both as a waveguide as well as sensing layer. When a monochromatic p- }}$ polarized light is sent to the transducer above the critical angle, this is coupled into the waveguiding layer via the evanescent wave created in the spacer. This arises when the propagation constants in the substrate and the waveguide match. The matching occurs at a very sharply defined angle, named the resonance angle. Upon binding of target molecules on the sensing layer the local refractive index changes, which modifies the resonance conditions. As a result, the resonance angle changes. The angular shift is proportional with the amount of biomolecules bound to the sensing surface.

When compared with conventional SPR technologies, the transducer used in RM approach allows a better light-matter interaction. As a result, the resonance angles are much sharper. Hence, one should expect better sensitivity of the method. Indeed, based on the available data, the sensitivity is slightly improved compared with SPR.

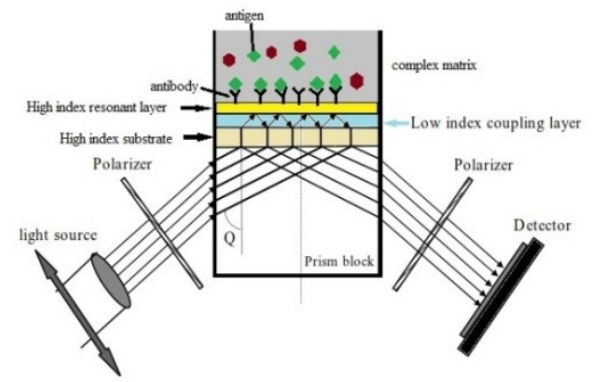

Figure 1-3 Illustration of the working principle of an RM biosensor. A polarized focused beam is sent, under total internal reflection conditions, to the transducer surface. The light couples in the waveguide layer via the evanescent wave. The resonance conditions change when the local refractive index on the sensing area changes, leading to a change in the angle of incidence [33]. RM biosensors have been used for over a decade $[34,35]$ and are available on the market [36].

\subsubsection{Interferometric biosensors}

The proof of principle of a Mach Zehnder Interferometer (MZI) (Figure 1-4a) for biosensing purposes was demonstrated by Heideman et al. [37]. Here, a coherent, polarized laser is employed. The light beam is sent to a $\mathrm{Y}$ junction of a waveguide 
structure and transmitted into two different arms: a sensing arm and a reference arm. The two branches recombine to produce an interference pattern and the signal is measured using a photodetector. When the refractive index of the medium in the sensing arm (due to analyte binding) changes, this leads to an optical phase shift, changing the light intensity detected by the photodetector.

(a)

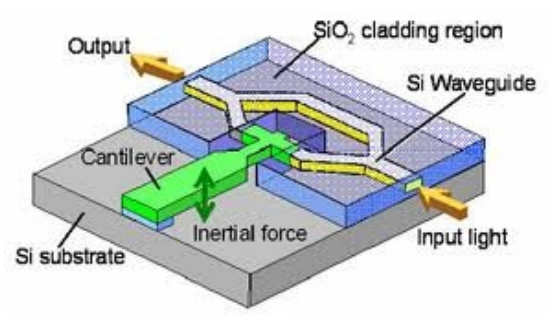

(b)

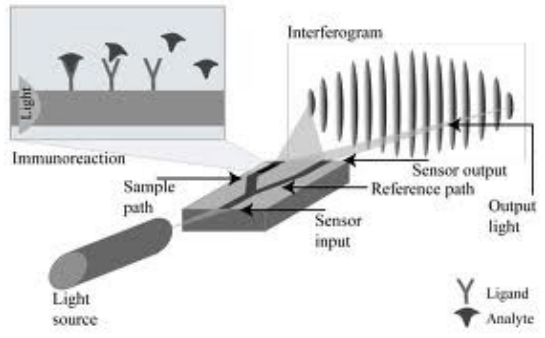

Figure 1-4 Schematic representation of some interferometric biosensors: (a) Mach Zehnder Interferometer sensor [38], (b) Young Interferometer biosensor [39]

The LOD determined by Heideman et al. for such a device was as low as $50 \mathrm{pM}$ for hCG molecules [37]. With a number of engineering improvements in the work done by Heideman and Lambeck the sensitivity of the method was improved [40].

A very similar biosensing platform is the Young interferometer (YI) as shown in Figure 1-4b. In this case, instead of recombining the arms at the output, the light output of the two arms forms an interference pattern on the detection screen. The first demonstration of a YI sensing device was shown by Brandenburg and Henninger [41], showing a LOD comparable with the maximum obtained in the case of the MZI biosensors. Several improvements in the design, proposed by Ymeti et al. [42, 43], allowed the possibility of multiplexing. The success of this method materialized in some commercial devices [44]. For example, the AnaLight from Fairfield Scientific was used to demonstrate the analysis of the binding interaction of biotin/streptavidin [45] and the detection of small molecules such as argininamide [46]. 


\subsubsection{Fiberoptics biosensors}

Traditionally fiber optics technology is used in the telecommunication field. They become attractive for biosensing owing to their small size, flexibility and efficient signal delivery $[47,48]$.

Most fiber optics biosensors rely on the total internal reflectance (TIR) concept. Here, the light transits the fiber by repeated reflectance on the cladding - core interface without losses. Different approaches have been developed to use in a maximized manner the resulting evanescent wave. Fiber Bragg gratings (FBG), long period gratings (LPG), and Fabry- Perot cavity based sensors are just a few examples of the available platforms.

FBG's (Figure 1-5a) are the most popular of all fiber optics sensing devices for refractive index detection [49]. A sensing surface is prepared by illuminating a decladded fiber with two focused intersecting laser beams that write a refractive index perturbation onto the fiber core, having a periodicity $(\Lambda)$ in the same order of magnitude as the wavelength of the light sources. The created structure reflects only a specific wavelength, called the Bragg wavelength $\left(\lambda_{\mathrm{B}}\right)$, according to the following relation: $\lambda_{\mathrm{B}}=\mathrm{n}_{\text {eff }} * \Lambda$, where $\mathrm{n}_{\text {eff }}$ is the effective refractive index experienced by the fiber core. Here, monitoring of $\lambda_{B}$ is used to detect changes in the refractive index due to binding events on the sensing surface.

To maximize the sensing capabilities several strategies have been used, including surface grating on a side of the fiber [50] or chemical etching of the fiber [51,52]. The last enhancement approach allowed the detection of $0.1 \mu \mathrm{M}$ of DNA target molecules with 20 base-pairs. Although this LOD is the least impressive compared to the above-mentioned technologies, it provides the first demonstration of the proof of principle of this sensing approach.

(a)

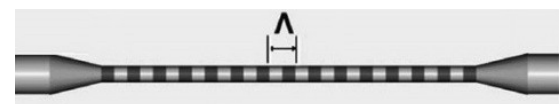

FBG sensor

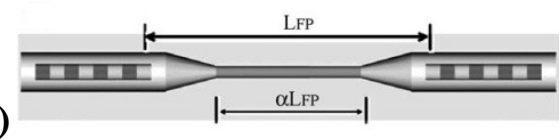

Figure 1-5 Schematic representation of several structures of fiber optics sensors. (a) FBG sensor[53]; (b) Fabry-Perot sensor [33] 
LPG's (Figure 1-5b) are an improved version of the FBG's by allowing a longer periodicity of the grating. Due to the larger grating pitch, they are easier to manufacture. Here too the chemical etching is used as an enhancement tactic for upgrading the sensitivity [54]. The earliest demonstration of the proof of principle in biomolecular detection was in 2000 by DeLissa et al. [52] for the detection of antibodies, where goat anti-human IgG antibody molecules were attached to the surface of the fiber and were used to detect human IgG in aqueous solution. The LOD determined was down to 30 nM. Later in 2007, Chen et al. used the etched LPG for the detection of haemoglobin [55, $56]$ being able to detect as low as 5*10-3\% of the haemoglobin in water. Fabry-Perot fiber sensors (Figure 1-5b) are usually a combination of two FBG's with a short piece of hollow fiber in between [57] or a tapered fiber [58,59]. For this type of device, an LOD of $76 \mu \mathrm{M}$ was determined for the detection of 26 base-pairs oligonucleotides [60].

Fiber optics sensors are attractive as they are cost-effective, compatible with standard optical fibers, simple design and small. However, their current performances in biochemical detection are far behind SPR or interferometric sensing platforms.

\section{Magnotech}

A new optical biosensor platform, based on magnetic nanoparticles, was developed at Philips Research, Netherlands [61], able to detect very low concentrations of analytes from raw samples.

The technology is based on magnetic nanoparticles detected optically in a stationary sample fluid. The system has two parts: (1) a disposable cartridge and (2) a handheld analyzer. The disposable cartridge is preloaded with functionalized magnetic particles, with a large field - induced magnetic moment, that are released in the solution once a drop of blood/ fluid has filled the cartridge. These particles will bind the target molecules from the blood. By introducing a magnetic field, the magnetic particles are attracted to the active area, where a second layer of receptor molecules (specific against the target molecules) is immobilized. This allows the specific binding of those magnetic particles that have at least one analyte molecule bound to their surface. Then a second 
magnetic field is introduced, in the opposite direction, leaving only the bound particles on the active surface. The rest of the nanoparticles are brought back in solution. The assay procedure is depicted in Figure 1-6a.

The final stage is the optical detection of the bound nanoparticles. This is realized using frustrated total internal reflectance (FTIR) (Figure 1-6b). A focused monochromatic beam illuminates the sensor surface under total internal reflectance conditions. When the sensor surface is empty, the light is totally reflected and collected by a photodetector. However, when magnetic particles are immobilized on the sensing area, due to specific binding, a part of the light reaching the sensing surface is absorbed and scattered by them. Thus, the reflected light collected by the photodetector is decreased. This change in the amount of the reflected light is proportional to the amount of bound magnetic particles.

(a)

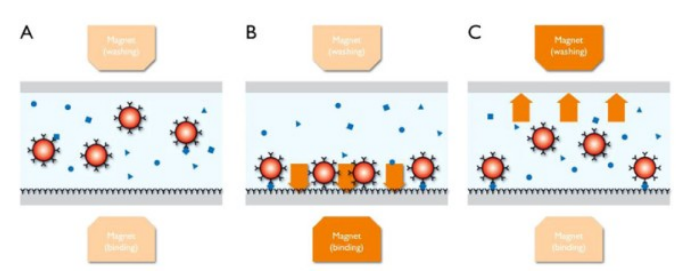

(b)

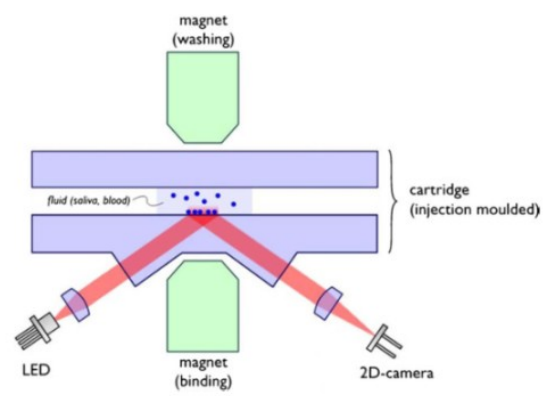

Figure 1-6 (a) The actuation process of the magnetic particles inside the cartridge during the immunoassay. A- The functionalized magnetic particles react with the target molecules and are carried through capillary flow towards the active area; B- A magnetic field attracts all the magnetic nanoparticles to the active zone, where only particles with bound target molecules will be locked on the surface; C- A second magnetic field, oriented in the opposite direction separates the nonbound magnetic particles from the active area. (b) The optical detection of bound magnetic particles by frustrated total internal reflectance (FTIR). Here the intensity of the reflected beam is decreased 
when magnetic particles adhere to the active area, due to scattering and absorption effects. The decrease is proportional with the amount of particles bound to the surface.

The system has been demonstrated in several assays such as the detection of cardiac troponin [62], parathyroid hormone [63] and in several drug abuse testing assays [64]. Although the results are preliminary, the concept presented by Philips Research show the possibility of fulfilling most of the sensor requirements like ease of use, low sample volume, and rapid detection.

\section{Nanoparticle based sensing strategies}

A disadvantage in label-free methods is the low sensitivity in the detection of small molecules due to the low refractive index change detected. One possible solution for this problem can be the use of an amplification step by using labels such as metal nanoparticles [65-67].

However, nanoparticles can also be used as sensor platforms [68-70]. Metallic nanoparticles are interesting for biosensing applications as they have the property of changing their optical properties in response to modifications of the characteristics of the local environment. This is possible due to the optical phenomenon of localized surface plasmon resonance (LSPR) [71]. This occurs when the nanoparticle interacts with the incident light at a specific wavelength, called resonance conditions. In these conditions, a coherent oscillation of the conduction electrons occurs leading to an enhancement of the scattering and absorption cross-sections [72]. For example, at resonance conditions, the light scattered by a $60 \mathrm{~nm} \mathrm{Au}$ particle is equivalent to the light emitted by $10^{5}$ fluorophores [65].

The optical properties of the nanoparticles, particularly the resonant wavelength, depend on their internal properties (size, shape, composition) and on the refractive index of the environment [73]. This dependence of the optical properties of the nanoparticle on the refractive index of the environment is the basis for refractive index sensing. This has been experimentally demonstrated in several papers [67, 73-75]. In addition, the small 
confinement of the LSPR field makes individual gold nanoparticles ideal candidates for the detection of small molecules.

There are several interrogation methods for the optical properties of gold nanoparticles: the monitoring of the extinction spectrum by means of a spectrometer (usually applied for aggregation assays) or the monitoring of the scattering spectrum of immobilized nanoparticles using Dark Field (DF) microscopy or Total Internal Reflectance. Scattering spectroscopy has the advantage of being able to provide the relevant optical information of individual nanoparticles.

In this sub-section, we will provide a short review on the detection methods for biosensing applications. First, we discuss aggregation assays and their performance, followed by a discussion of the assays where individual nanoparticles are interrogated and of the detection platforms employed.

\subsection{Aggregation-based biosensing strategies}

Here, metal nanoparticles are functionalized with receptor molecules, and by exposing them to analyte molecules, they will aggregate as shown in Figure 1-7.

The aggregation can be measured using an UV-VIS spectrometer as previously done by Yguerabide et al. [66]. The result can also be easily observed with the naked eye as the aggregation results in a visible colour change of the solution. The method employed was simple, fast and reliable needing only a spectrometer and little training. However, it requires high volumes of analyte and the LOD is just below the nanomolar range (0.1-0.2 $\mathrm{nM}$ for thrombin in plasma) $[76,77]$. 


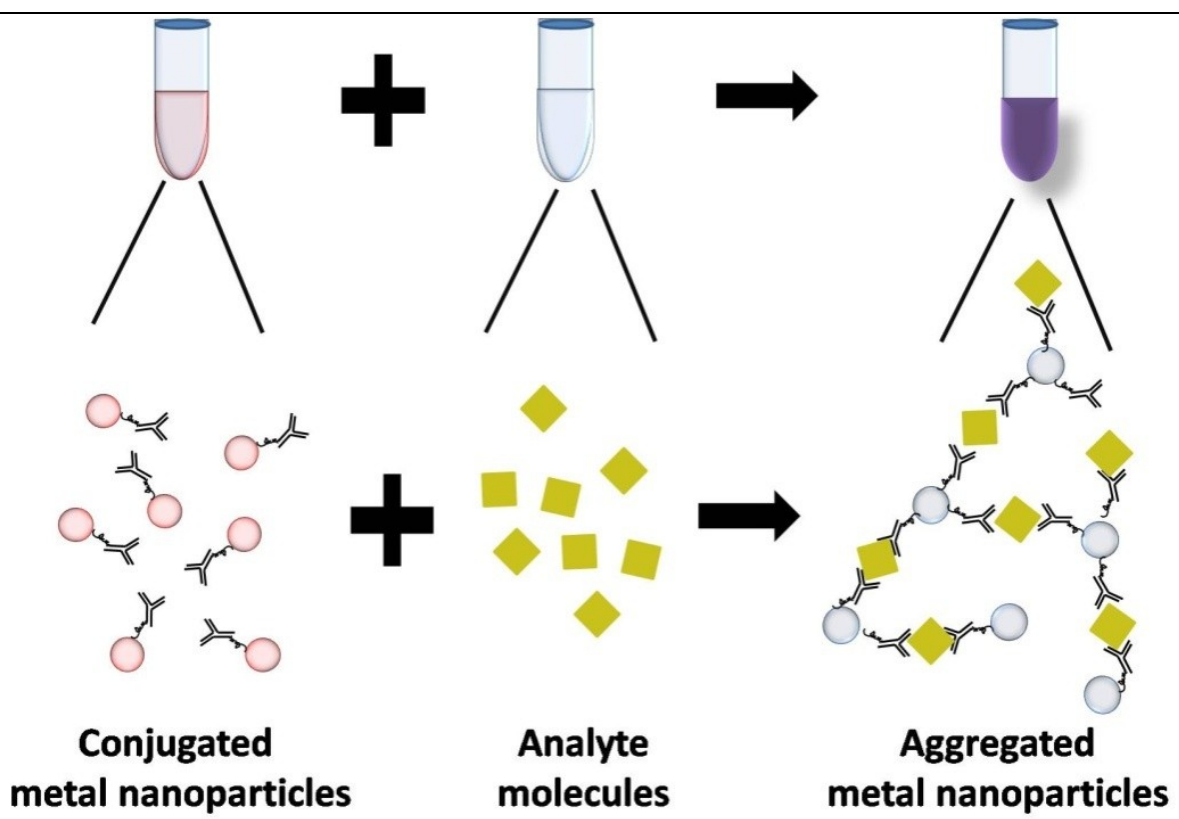

Figure 1-7 Schematic representation of an aggregation assay. (a) Functionalized particles exposed to (b) analyte molecules will lead to immuno-binding/hybridization resulting in a network of conjugated nanoparticles linked to each other by the analyte molecules. The colour of the solution changes due to aggregation of the conjugates.

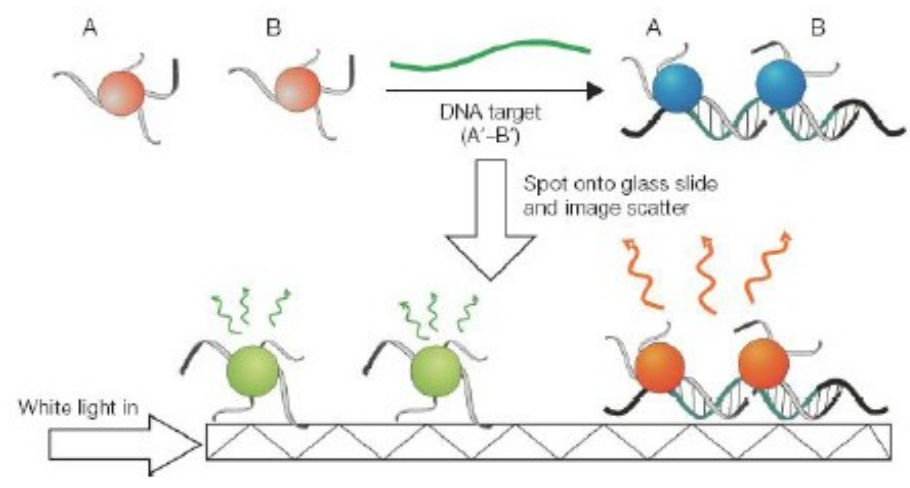

Figure 1-8 Colorimetric detection of DNA hybridization. DNA functionalized nanoparticles are hybridized in solution with a DNA target molecule. The sample is then spotted on an illuminated glass slide and the scattered light is visualized [97].

Another approach is to move the assay onto a substrate. First, the reaction is done in solution, and then the measurement of the signal is done on a surface. For 
$\overline{\text { example, Storhoff et al. were able to detect as low as } 20 \text { pM of a DNA strand by spotting }}$ the hybridized solution on an illuminated waveguide glass [79]. The detection principle is based on measuring the light scattered by the aggregated nanoparticles spotted on the surface, as seen in Figure 1-8. The results presented in this work showed an increase in sensitivity by four orders of magnitude when compared with classical aggregation methods.

Another type of particle aggregation assay exploits the intense coupling effect when the distance between two linked nanoparticles is changed upon a binding event [79]. In this assay, the inter-particle distance between two nanoparticles linked by a singlestranded DNA can be varied by hybridization with target molecules of different lengths. The inter-particle distance can be experimentally determined by analysing the colour of the resulting dimers by measuring the extinction spectrum [80].

The perfect example of the commercial application of the optical properties of nanoparticles is the pregnancy test. Here, the presence of the pregnancy biomarker, human chorionic gonadotrophin (hCG) hormone is detected in urine. This hormone binds specifically to mouse anti-hCG. This is both conjugated on a gold nanoparticle that scatters red light and immobilized on the test lane. The hormone, when present in the tested urine, binds to the conjugated particles. Through capillary forces is brought to the testing area, where it attaches to the immobilized antibodies changing the color to red [81].

These aggregation assays are widely used and can be employed in complex biological media without prior sample preparation. However, in all these cases, single binding events cannot be observed.

\subsection{Biosensors based on the LSPR monitoring of individual nanoparticles}

As previously mentioned the scattering of individual nanoparticles immobilized on a surface can be easily observed in a darkfield or TIR setup. Gunnarson et al. proposed a simple single molecule read-out system using labeled vesicles modified with DNA 
$\overline{\text { molecules. In their experiments, a glass slide covered with a certain number of receptor }}$ molecules was exposed to various concentrations of analytes. The vesicles functionalized with the 'receptor' molecule act as labels and bind only where analyte molecules are present, and each bound particle corresponds to one binding event. In principle by counting the bound particles, and with previous knowledge of the receptor density present on the glass slide, a concentration can be determined. The detection limit using this approach is in the $\mathrm{fM}$ range [82]. The disadvantage of this approach is the unknown number of receptor sites.

As an alternative, individual gold nanoparticles are immobilized on a surface via chemical linkers or prepared by nanolithography. The nanoparticles are then functionalized with a biological molecule, with the role of recognition site for analyte molecules. The immediate advantage of this approach is that, because nanoparticles are visible in a DF setup, the receptor sites can be easily estimated from the available nanoparticles. The obtained sensor is then exposed to the analyte molecule and when binding events occur, a change in the optical properties of the nanoparticles will be recorded. Figure 1-9 depicts such a sensing approach.

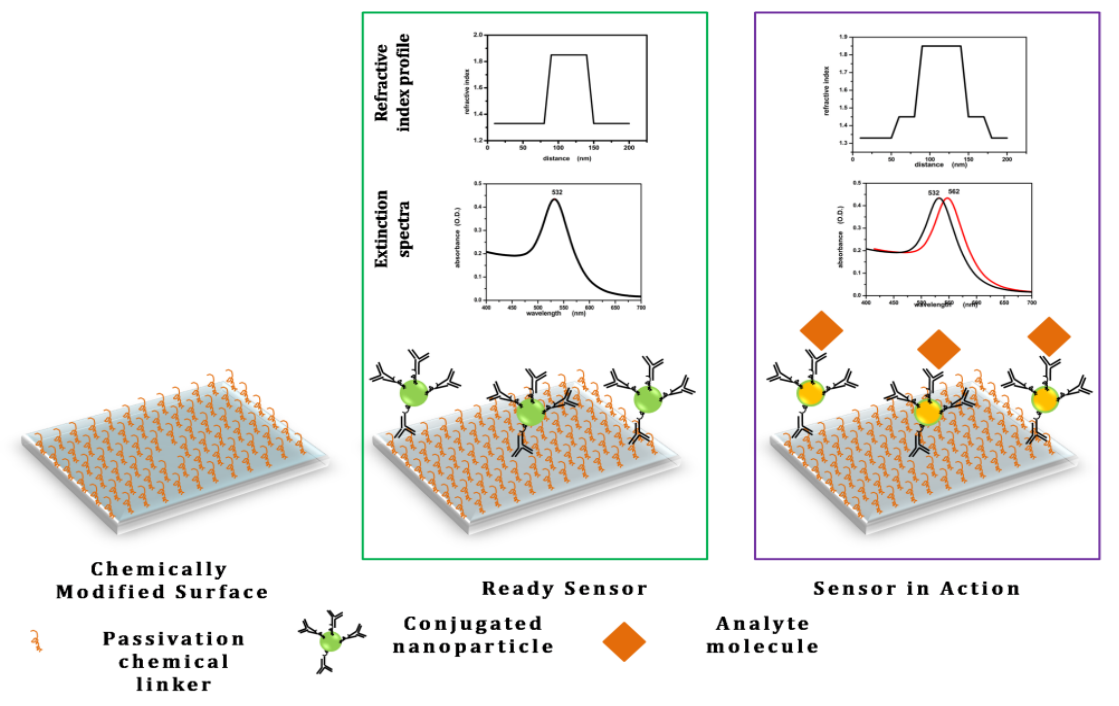

Figure 1-9 Schematic representation of a nanoparticle - based sensing approach. The surface is chemically modified (a) to bind covalently/by adsorption the functionalized nanoparticles (b). The 
ready-to-use sensor is then introduced in a transducing system (e.g. DF microscope, TIR) and the signal (e.g. scattering spectrum, colour) of individual nanoparticles is measured. In the beginning of the assay, the nanoparticle is characterized by a certain local refractive index determined by the molecules of the surrounding medium (e.g. buffer, water). When a binding event occurs, the local refractive index around the nanoparticles changes as the analyte molecule replaces one or more molecules of the surrounding medium. As a result, the optical properties of the nanoparticle change and a shifted signal is measured.

The first test of this type of assay was performed on a biotin - streptavidin model couple. Here, the immobilized gold nanoparticle was functionalized via a chemical linker with biotin molecules. The binding of the streptavidin molecules was monitored by measuring the intensity of the scattered light by using a flatbed scanner [83], or the spectral changes measured with a UV-VIS spectrometer [84]. The minimum LOD that could be achieved using this method was $0.094 \mathrm{nM}$ in PBS and $19 \mathrm{nM}$ in serum [102]. The major disadvantage is that the behaviour of individual nanoparticles is lost as the measured signal is an average of the signals of all nanoparticles.

Measurements of the scattering spectra of individual nanoparticles were reported for the first time by the group of Van Duyne [85], [86], [87]. In a TIR setup, they detected the binding of anti-biotin molecules to the biotin-functionalized individual Ag particle. They monitored changes in the scattering spectrum of individual nanoparticles and were able to reach an LOD of $0.7 \mathrm{nM}$ [88]. By optimisation of the characteristics of the nanoparticles an impressive zeptomole detection limit was reached [86].

Single binding events can be detected if amplification is used. By bringing in close proximity two particles, their electromagnetic coupling will lead to an enhanced red shifting of the LSPR peak. In their work, Sannomiya et al. [89] showed evidence that single binding events can be observed and that the LSPR peak can be monitored using a simple spectrometer. However, spectrometers do not allow the simultaneous monitoring of many individual nanoparticles. In order to determine low concentrations, a detection method should be capable of measuring very low receptor coverage, which is only possible when a large number of receptor sites can be monitored $\left(\sim 10^{4}-10^{5}\right)$. Therefore, a high level of parallelization is important. By using special filters such as liquid crystal 
tunable filters) in a TIR or DF setup, one could measure the spectra of several up to hundred particles in one field of view [90], [91]. Although they provide reliable information, the parallelization capabilities of such a method are very limited. In addition, the implementation costs of such a filter are relatively high.

\section{Conclusions}

Research in the optical sensing area has allowed the development of new devices and improvements in the classical methods. The main efforts were put into obtaining improved sensitivity of the sensing device, by increasing the transducer sensitivity. However, research is carried out also in improving the chemical/biological activity of the receptor sites, as well as system integration. All these could result in the emerging of new biosensing technologies with upgraded characteristics.

\section{Outline of the thesis}

In this thesis, we introduce a new optical method based on gold nanoparticles as individual sensing platforms for the detection of low concentrations of analytes (DNA or proteins). Here we provide the proof of principle of the methodology in the detection of immunoreactions and determine its limit of detection. We show that with a simple colour camera we are able to detect simultaneously immunobinding on thousands of individual gold nanoparticles [92], by measuring the change in the colour of many individual nanoparticles [93]. If an amplification step is used the LOD of such a system can be boosted even more [94].

In order to get the best sensing strategies an understanding of the physical background is needed. Chapter 2 presents a review of the physical aspects of LSPR and continues with a theoretical discussion on the sensing strategies employing individual gold nanoparticles as sensing platforms. The concept of parallel detection of multiple individually addressable nanoparticles is introduced and a theoretical LOD is determined. 
Chapter 3 introduces a new detection method of binding events using a colour camera in a DF setup. The feasibility of this approach was tested in an adsorption assay and an immunoassay and the LOD of the method for this sensing strategy was experimentally measured. In addition, two different detection approaches are tested and their performances compared.

In Chapter 4, we introduce a new sensing strategy using gold nanoparticle probes in microfluidic cells in colorimetric darkfield microscopy enabling the simultaneous sensing of hundreds of binding events of individual particles simultaneously. Here, single binding events can be observed and the results were confirmed by independent methods like Scanning Electron Microscopy.

After demonstrating the proof of principle of our detection method in an unamplified protein assay and direct immunoassay, in Chapter 5 we test our setup in an amplified protein assay and determine the performances of the setup by measuring experimentally the LOD of the system.

In Chapter 6, we review the main achievements presented in this thesis, and we present several future recommendations for this line of research. 


\section{References}

[1] L. Frances and T. Chris, eds., Optical biosensors- Today and Tomorrow, Elsevier B.V., 2008.

[2] H.M. Abraham, E. Albone, G.P. Berrie, U. Bilitewski, H. Breer, K. Cammann, S.-M. Chang, A. D’Amico, B. Danielsson, and C. Di Natale, Handbook of Biosensors and Electronic Noses Medicine, Food and the Environment, CRC Press, 1997.

[3] G. Urban, H. Kamper, A. Jachimowicz, F. Kohl, H. Kuttner, F. Olcaytug, P. Goiser, F. Pittner, T. Schalkhammer, and E. Mann-Buxbaum, "The construction of microcalorimetric biosensors by use of high resolution thin-film thermistors," Biosensors and Bioelectronics, vol. 6, 1991, pp. $275-280$.

[4] M. Pohanka and P. Skládal, "Piezoelectric Immunosensor for Francisella tularensis Detection Using Immunoglobulin M in a Limiting Dilution," Analytical Letters, vol. 38, Feb. 2005, pp. 411-422.

[5] M. Pohanka, P. Skládal, and O. Pavliš, "Label-Free Piezoelectric Immunosensor for Rapid Assay of Escherichia coli," Journal of Immunoassay and Immunochemistry, vol. 29, Dec. 2007, pp. 70-79.

[6] H. Chen, J.-H. Jiang, Y.-F. Li, T. Deng, G.-L. Shen, and R.-Q. Yu, “A novel piezoelectric immunoagglutination assay technique with antibody-modified liposome," Biosensors and Bioelectronics, vol. 22, Jan. 2007, pp. 993-999.

[7] A.L. Ghindilis, P. Atanasov, M. Wilkins, and E. Wilkins, "Immunosensors: electrochemical sensing and other engineering approaches," Biosensors and Bioelectronics, vol. 13, Jan. 1998, pp. 113-131.

[8] B.S. Ferguson, S.F. Buchsbaum, J.S. Swensen, K. Hsieh, X. Lou, and H.T. Soh, "Integrated Microfluidic Electrochemical DNA Sensor," Analytical Chemistry, vol. 81, Jul. 2009, pp. 6503-6508.

[9] Y. Zhang, Y. Wang, H. Wang, J.-H. Jiang, G.-L. Shen, R.-Q. Yu, and J. Li, "Electrochemical DNA Biosensor Based on the Proximity-Dependent Surface Hybridization Assay," Analytical Chemistry, vol. 81, Jan. 2009, pp. 1982-1987. 
[10] M. De, S. Rana, H. Akpinar, O.R. Miranda, R.R. Arvizo, U.H.F. Bunz, and V.M. Rotello, "Sensing of proteins in human serum using conjugates of nanoparticles and green fluorescent protein," Nat Chem, vol. 1, Sep. 2009, pp. 461-465.

[11] B. Yin, R.M. Whyatt, F.P. Perera, M.C. Randall, T.B. Cooper, and R.M. Santella, "Determination of 8-hydroxydeoxyguanosine by an immunoaffinity chromatography-monoclonal antibody-based ELISA,” Free Radical Biology and Medicine, vol. 18, Jun. 1995, pp. 1023-1032.

[12] M. Vandermeeren, M. Mercken, E. Vanmechelen, J. Six, A. Van de Voorde, J.-J. Martin, and P. Cras, "Detection of Proteins in Normal and Alzheimer's Disease Cerebrospinal Fluid with a Sensitive Sandwich Enzyme-Linked Immunosorbent Assay," Journal of Neurochemistry, vol. 61, 1993, pp. 1828-1834.

[13] A. Dagerlind, K. Friberg, A.J. Bean, and T. Hökfelt, "Sensitive mRNA detection using unfixed tissue: combined radioactive and non-radioactive in situ hybridization histochemistry," Histochemistry and Cell Biology, vol. 98, pp. 39-49.

[14] X. Michalet, F.F. Pinaud, L.A. Bentolila, J.M. Tsay, S. Doose, J.J. Li, G. Sundaresan, A.M. Wu, S.S. Gambhir, and S. Weiss, "Quantum Dots for Live Cells, in Vivo Imaging, and Diagnostics," Science, vol. 307, 2005, pp. 538-544.

[15] R. Gill, M. Zayats, and I. Willner, "Semiconductor Quantum Dots for Bioanalysis," Angewandte Chemie International Edition, vol. 47, 2008, pp. 7602-7625.

[16] X. Wu, H. Liu, J. Liu, K.N. Haley, J.A. Treadway, J.P. Larson, N. Ge, F. Peale, and M.P. Bruchez, "Immunofluorescent labeling of cancer marker Her2 and other cellular targets with semiconductor quantum dots," Nat Biotech, vol. 21, Jan. 2003, pp. 41-46.

[17] H. Liao, C.L. Nehl, and J.H. Hafner, "Biomedical applications of plasmon resonant metal nanoparticles," Nanomedicine, vol. 1, Aug. 2006, pp. 201-208.

[18] X.X. Han, Y. Kitahama, T. Itoh, C.X. Wang, B. Zhao, and Y. Ozaki, "Protein-Mediated Sandwich Strategy for Surface-Enhanced Raman Scattering: Application to Versatile Protein Detection," Analytical Chemistry, vol. 81, Apr. 2009, pp. 3350-3355.

[19] E.V. Suprun, A.L. Shilovskaya, A.V. Lisitsa, T.V. Bulko, V.V. Shumyantseva, and A.I. Archakov, "Electrochemical Immunosensor Based on Metal Nanoparticles for Cardiac Myoglobin Detection in Human Blood Plasma," Electroanalysis, vol. 23, 2011, pp. 1051-1057. 
[20] K.B. Mogensen, J. El-Ali, A. Wolff, and J.P. Kutter, "Integration of Polymer Waveguides for Optical Detection in Microfabricated Chemical Analysis Systems," Appl. Opt., vol. 42, Jul. 2003, pp. 4072-4079.

[21] B. Liedberg, C. Nylander, and I. Lunstrom, "Surface plasmon resonance for gas detection and biosensing," Sensors and Actuators, vol. 4, 1983, pp. 299-304.

[22] "http://www.biacore.com/lifesciences/products/systems_overview/index.html."

[23] "http://www.ibis-spr.nl/."

[24] "http://www.sensata.com/sensors/spreeta-analytical-sensor-lights.htm."

[25] J. Homola, S.S. Yee, and G. Gauglitz, "Surface plasmon resonance sensors: review," Sensors and Actuators B: Chemical, vol. 54, Jan. 1999, pp. 3-15.

[26] J. Homola, "Present and future of surface plasmon resonance biosensors," Analytical and Bioanalytical Chemistry, vol. 377, pp. 528-539.

[27] J. Homola, "Surface Plasmon Resonance Sensors for Detection of Chemical and Biological Species," Chemical Reviews, vol. 108, 2008, pp. 462-493.

[28] J. Dostalek, H. Vaisocherova, and J. Homola, "Multichannel surface plasmon resonance biosensor with wavelength division multiplexing," Sensors and Actuators B: Chemical, vol. 108, 2005, pp. $758-764$.

[29] H.J. Lee, D. Nedelkov, and R.M. Corn, "Surface Plasmon Resonance Imaging Measurements of Antibody Arrays for the Multiplexed Detection of Low Molecular Weight Protein Biomarkers," Analytical Chemistry, vol. 78, Aug. 2006, pp. 6504-6510.

[30] Y. Li, H.J. Lee, and R.M. Corn, "Detection of Protein Biomarkers Using RNA Aptamer Microarrays and Enzymatically Amplified Surface Plasmon Resonance Imaging," Analytical Chemistry, vol. 79, 2007, pp. 1082-1088.

[31] X. Yao, X. Li, F. Toledo, C. Zurita-Lopez, M. Gutova, J. Momand, and F. Zhou, "Subattomole oligonucleotide and p53 cDNA determinations via a high-resolution surface plasmon resonance combined with oligonucleotide-capped gold nanoparticle signal amplification," Analytical Biochemistry, vol. 354, 2006, pp. 220-228. 
[32] F. Song, F. Zhou, J. Wang, N. Tao, J. Lin, R.L. Vellanoweth, Y. Morquecho, and J. Wheeler Laidman, "Detection of oligonucleotide hybridization at femtomolar level and sequence-specific gene analysis of the Arabidopsis thaliana leaf extract with an ultrasensitive surface plasmon resonance spectrometer," Nucleic Acids Research, vol. 30, 2002, p. e72.

[33] X. Fan, I.M. White, S.I. Shopova, H. Zhu, J.D. Suter, and Y. Sun, "Sensitive optical biosensors for unlabeled targets: A review," Analytica Chimica Acta, vol. 620, 2008, pp. 8-26.

[34] R. Cush, J.M. Cronin, W.J. Stewart, C.H. Maule, J. Molloy, and N.J. Goddard, "The resonant mirror: a novel optical biosensor for direct sensing of biomolecular interactions Part I: Principle of operation and associated instrumentation," Biosensors and Bioelectronics, vol. 8, 1993, pp. 347-354.

[35] P.E. Buckle, R.J. Davies, T. Kinning, D. Yeung, P.R. Edwards, D. Pollard-Knight, and C.R. Lowe, "The resonant mirror: a novel optical sensor for direct sensing of biomolecular interactions part II: applications,” Biosensors and Bioelectronics, vol. 8, 1993, pp. 355-363.

[36] "www.neosensors.com."

[37] R.G. Heideman, R.P.H. Kooyman, and J. Greve, "Performance of a highly sensitive optical waveguide Mach-Zehnder interferometer immunosensor," Sensors and Actuators B: Chemical, vol. 10, Feb. 1993, pp. 209-217.

[38] M. Suzuki, G. Kawai, H. Izumi, S. Aoyagi, and S. Yokoyama, "Design and Simulation of Inertial Force Sensor Using Mach-Zehnder Inter- ferometer with Optical Waveguides Made of Crystal Silicon," IEEJ Transaction SM, vol. 129, 2009, pp. 301-306.

[39] "http://www.photonics4life.eu/index.php/Consortium/P4L-DB/All-items/IntegratedYoung-interferometer-sensors-for-biosensing/\%28version $\% 29 / 2 . "$

[40] R.G. Heideman and P.V. Lambeck, "Remote opto-chemical sensing with extreme sensitivity: design, fabrication and performance of a pigtailed integrated optical phase-modulated Mach-Zehnder interferometer system," Sensors and Actuators B: Chemical, vol. 61, 1999, pp. 100-127.

[41] A. Brandenburg and R. Henninger, "Integrated optical Young interferometer," Appl. Opt., vol. 33, Sep. 1994, pp. 5941-5947. 
[42] A. Ymeti, J.S. Kanger, J. Greve, G.A.J. Besselink, P.V. Lambeck, R. Wijn, and R.G. Heideman, "Integration of microfluidics with a four-channel integrated optical Young interferometer immunosensor," Biosensors and Bioelectronics, vol. 20, 2005, pp. 1417-1421.

[43] R.R. Wijn, R.G. Heideman, V. Subramaniam, and J.S. Kanger, with A. Ymeti, J. Greve, P.V. Lambeck, and T. Wink, van Hövell and Beumer, "Fast, Ultrasensitive Virus Detection Using a Young Interferometer Sensor,” Nano Letters, vol. 7, 2007, pp. 394-397.

[44] "http://www.farfield-scientific.com."

[45] G.H. Cross, A.A. Reeves, S. Brand, J.F. Popplewell, L.L. Peel, M.J. Swann, and N.J. Freeman, "A new quantitative optical biosensor for protein characterisation," Biosensors and Bioelectronics, vol. 19, 2003, pp. 383-390.

[46] V. Özalp, "Dual-polarization interferometry for quantification of small molecules using aptamers," Analytical and Bioanalytical Chemistry, vol. 402, 2012, pp. 799-804 LA - English.

[47] E. Udd, “An overview of fiber-optic sensors," Review of Scientific Instruments, vol. 66, 1995, pp. 4015-4030.

[48] A. Brecht and G. Gauglitz, "Recent developments in optical transducers for chemical or biochemical applications," Sensors and Actuators B: Chemical, vol. 38, 1997, pp. 1-7.

[49] O.S. Wolfbeis, "Fiber-Optic Chemical Sensors and Biosensors," Analytical Chemistry, vol. 76, 2004, pp. 3269-3284.

[50] A. Boltasseva, S.I. Bozhevolnyi, T. Nikolajsen, and K. Leosson, "Compact Bragg Gratings for Long-Range Surface Plasmon Polaritons," J. Lightwave Technol., vol. 24, Feb. 2006, p. 912.

[51] A.N. Chryssis, S.S. Saini, S.M. Lee, H. Yi, W.E. Bentley, and M. Dagenais, "Detecting hybridization of DNA by highly sensitive evanescent field etched core fiber Bragg grating sensors," IEEE Journal of Selected Topics in Quantum Electronics, vol. 11, 2005, pp. 864-872.

[52] M.P. DeLisa, Z. Zhang, M. Shiloach, S. Pilevar, C.C. Davis, J.S. Sirkis, and W.E. Bentley, "Evanescent Wave Long-Period Fiber Bragg Grating as an Immobilized Antibody Biosensor," Analytical Chemistry, vol. 72, 2000, pp. 2895-2900. 
[53] W. Liang, Y. Huang, Y. Xu, R.K. Lee, and A. Yariv, "Highly sensitive fiber Bragg grating refractive index sensors," Applied Physics Letters, vol. 86, 2005.

[54] L. Rindorf, J.B. Jensen, M. Dufva, L.H. Pedersen, P.E. Høiby, and O. Bang, "Photonic crystal fiber long-period gratings for biochemical sensing," Opt. Express, vol. 14, Sep. 2006, pp. 8224-8231.

[55] X. Chen, K. Zhou, L. Zhang, and I. Bennion, "Dual-peak long-period fiber gratings with enhanced refractive index sensitivity by finely tailored mode dispersion that uses the light cladding etching technique," Appl. Opt., vol. 46, Feb. 2007, pp. 451-455.

[56] X. Chen, L. Zhang, K. Zhou, E. Davies, K. Sugden, I. Bennion, M. Hughes, and A. Hine, "Real-time detection of DNA interactions with long-period fiber-grating-based biosensor," Opt. Lett., vol. 32, Sep. 2007, pp. 2541-2543.

[57] R. Ballerstadt and J.S. Schultz, "A Fluorescence Affinity Hollow Fiber Sensor for Continuous Transdermal Glucose Monitoring," Analytical Chemistry, vol. 72, 2000, pp. 4185-4192.

[58] K. Rijal, A. Leung, P.M. Shankar, and R. Mutharasan, "Detection of pathogen Escherichia coli O157:H7 AT 70 cells/mL using antibody-immobilized biconical tapered fiber sensors," Biosensors and Bioelectronics, vol. 21, 2005, pp. 871-880.

[59] Z.L. Ran, Y.J. Rao, W.J. Liu, X. Liao, and K.S. Chiang, "Laser-micromachined FabryPerot optical fiber tip sensor for high-resolution temperature-independent measurement of refractive index," Opt. Express, vol. 16, Feb. 2008, pp. 2252-2263.

[60] X. Wang, K.L. Cooper, A. Wang, J. Xu, Z. Wang, Y. Zhang, and Z. Tu, "Label-free DNA sequence detection using oligonucleotide functionalized optical fiber," Applied Physics Letters, vol. 89, 2006.

[61]“http://www.newscenter.philips.com/main/standard/news/backgrounders/2010/20100107_ magnetic_biosensor.wpd.”

[62] D.M. Bruls, T.H. Evers, J.A.H. Kahlman, P.J.W. van Lankvelt, M. Ovsyanko, E.G.M. Pelssers, J.J.H.B. Schleipen, F.K. de Theije, C.A. Verschuren, T. van der Wijk, J.B.A. van Zon, W.U. Dittmer, A.H.J. Immink, J.H. Nieuwenhuis, and M.W.J. Prins, "Rapid integrated biosensor for multiplexed immunoassays based on actuated magnetic nanoparticles," Lab on a Chip, 2009, p. 3504-3510. 
[63] V. Jarrige, J.H. Nieuwenhuis, J.P.H.F. van Son, M.F.W.C. Martens, and J.L.M. Vissers, “A fast intraoperative PTH point-of-care assay on the Philips handheld magnotech system," Langenbecks Arch Surg, 2011, p. 337-343.

[64] W.U. Dittmer, P. de Kievit, M.W.J. Prins, J.L.M. Vissers, M.E.C. Mersch, and M.F.W.C. Martens, "Sensitive and rapid immunoassay for parathyroid hormone using magnetic particle labels and magnetic actuation," Journal of Immunological Methods, 2008, pp. 40-46.

[65] J. Yguerabide and E.E. Yguerabide, "Light-Scattering Submicroscopic Particles as Highly Fluorescent Analogs and Their Use as Tracer Labels in Clinical and Biological Applications: I. Theory," Analytical Biochemistry, vol. 262, Sep. 1998, pp. 137-156.

[66] J. Yguerabide and E.E. Yguerabide, "Light-Scattering Submicroscopic Particles as Highly Fluorescent Analogs and Their Use as Tracer Labels in Clinical and Biological Applications: II. Experimental Characterization," Analytical Biochemistry, vol. 262, Sep. 1998, pp. 157-176.

[67] I.O. Sosa, C. Noguez, and R.G. Barrera, "Optical Properties of Metal Nanoparticles with Arbitrary Shapes," The Journal of Physical Chemistry B, vol. 107, Jun. 2003, pp. 6269-6275.

[68] A.W. Wark, H.J. Lee, A.J. Qavi, and R.M. Corn, "Nanoparticle-Enhanced Diffraction Gratings for Ultrasensitive Surface Plasmon Biosensing," Analytical Chemistry, vol. 79, 2007, pp. 6697-6701.

[69] S. Fang, H.J. Lee, A.W. Wark, and R.M. Corn, “Attomole Microarray Detection of MicroRNAs by Nanoparticle-Amplified SPR Imaging Measurements of Surface Polyadenylation Reactions," Journal of the American Chemical Society, vol. 128, Oct. 2006, pp. 14044-14046.

[70] J.A. Hansen, R. Mukhopadhyay, J.Ø. Hansen, and K.V. Gothelf, "Femtomolar Electrochemical Detection of DNA Targets Using Metal Sulfide Nanoparticles," Journal of the American Chemical Society, vol. 128, 2006, pp. 3860-3861.

[71] L. Kelly, E. Coronado, L. Zhao, and G. Schatz, "The Optical Properties of Metal Nanoparticles: The Influence of Size, Shape, and Dielectric Environment," The Journal of Physical Chemistry B, vol. 107, Jan. 2003, pp. 668-677.

[72] H.C. van de Hulst, Light Scattering by small particles, New York: 1981. 
[73] L.A. Trachuk, A.G. Melnikov, and N.G. Khlebtsov, "Dependence of the optical properties of metal nanoparticles on the external dielectric medium: effects of the particle size, shape, and structure," SPIE Proceedings, vol. 5829, 2005, pp. 127-137.

[74] G. Raschke, S. Kowarik, T. Franzl, C. Sönnichsen, T.A. Klar, J. Feldmann, A. Nichtl, and K. Kürzinger, "Biomolecular Recognition Based on Single Gold Nanoparticle Light Scattering," Nano Letters, vol. 3, May. 2003, pp. 935-938.

[75] C. Noguez, "Surface Plasmons on Metal Nanoparticles: The Influence of Shape and Physical Environment," The Journal of Physical Chemistry C, vol. 111, Feb. 2007, pp. 3806-3819.

[76] X. Xie, W. Xu, T. Li, and X. Liu, "Colorimetric Detection of HIV-1 Ribonuclease H Activity by Gold Nanoparticles," Small, vol. 7, 2011, pp. 1393-1396.

[77] C.-K. Chen, C.-C. Huang, and H.-T. Chang, "Label-free colorimetric detection of picomolar thrombin in blood plasma using a gold nanoparticle-based assay," Biosensors and Bioelectronics, vol. 25, 2010, pp. 1922-1927.

[78] J.J. Storhoff, A.D. Lucas, V. Garimella, Y.P. Bao, and U.R. Muller, "Homogeneous detection of unamplified genomic DNA sequences based on colorimetric scatter of gold nanoparticle probes," Nat Biotech, vol. 22, Jul. 2004, pp. 883-887.

[79] C. Sonnichsen, B.M. Reinhard, J. Liphardt, and A.P. Alivisatos, “A molecular ruler based on plasmon coupling of single gold and silver nanoparticles," Nat Biotech, vol. 23, Jun. 2005, pp. 741-745.

[80] S.J. Hurst, M.S. Han, A.K.R. Lytton-Jean, and C.A. Mirkin, "Screening the Sequence Selectivity of DNA-Binding Molecules Using a Gold Nanoparticle-Based Colorimetric Approach,” Analytical Chemistry, vol. 79, 2007, pp. 7201-7205.

[81] S. Assadollahi, C. Reininger, R. Palkovits, P. Pointl, and T. Schalkhammer, "From Lateral Flow Devices to a Novel Nano-Color Microfluidic Assay," Sensors, vol. 9, 2009, pp. 6084-6100.

[82] A. Gunnarsson, P. Jonsson, R. Marie, J.O. Tegenfeldt, and F. Hook, "Single-Molecule Detection and Mismatch Discrimination of Unlabeled DNA Targets," Nano Letters, vol. 8, 2008, pp. 183-188. 
[83] N. Nath and A. Chilkoti, "Label-Free Biosensing by Surface Plasmon Resonance of Nanoparticles on Glass: Optimization of Nanoparticle Size," Analytical Chemistry, vol. 76, 2004, pp. $5370-5378$.

[84] S.M. Marinakos, S. Chen, and A. Chilkoti, "Plasmonic Detection of a Model Analyte in Serum by a Gold Nanorod Sensor," Analytical Chemistry, vol. 79, 2007, pp. 5278-5283.

[85] A.J. Haes and R.P. Van Duyne, "A Nanoscale Optical Biosensor: Sensitivity and Selectivity of an Approach Based on the Localized Surface Plasmon Resonance Spectroscopy of Triangular Silver Nanoparticles," Journal of the American Chemical Society, vol. 124, Aug. 2002, pp. 10596-10604.

[86] A.D. McFarland and R.P. Van Duyne, "Single Silver Nanoparticles as Real-Time Optical Sensors with Zeptomole Sensitivity,” Nano Letters, vol. 3, Jul. 2003, pp. 1057-1062.

[87] M.D. Malinsky, K.L. Kelly, G.C. Schatz, and R.P. Van Duyne, "Chain Length Dependence and Sensing Capabilities of the Localized Surface Plasmon Resonance of Silver Nanoparticles Chemically Modified with Alkanethiol Self-Assembled Monolayers," Journal of the American Chemical Society, vol. 123, Jan. 2001, pp. 1471-1482.

[88] J.C. Riboh, A.J. Haes, A.D. McFarland, C. Ranjit Yonzon, and R.P. Van Duyne, “A Nanoscale Optical Biosensor: Real-Time Immunoassay in Physiological Buffer Enabled by Improved Nanoparticle Adhesion," The Journal of Physical Chemistry B, vol. 107, Jan. 2003, pp. 17721780 .

[89] T. Sannomiya, C. Hafner, and J. Voros, "In situ Sensing of Single Binding Events by Localized Surface Plasmon Resonance,” Nano Letters, vol. 8, Sep. 2008, pp. 3450-3455.

[90] C.L. Baciu, J. Becker, A. Janshoff, and C. Sönnichsen, "Protein-Membrane Interaction Probed by Single Plasmonic Nanoparticles,” Nano Letters, vol. 8, May. 2008, pp. 1724-1728.

[91] S. Chen, M. Svedendahl, R.P.V. Duyne, and M. Kall, "Plasmon-Enhanced Colorimetric ELISA with Single Molecule Sensitivity,” Nano Letters, vol. 11, Mar. 2011, pp. 1826-1830.

[92] F. Ungureanu, J. Halamek, R. Verdoold, and R.P.H. Kooyman, "The use of a colour camera for quantitative detection of protein binding nanoparticles," Proc. SPIE 7192, vol. 71920O, 2009. 
[93] F. Ungureanu, D. Wasserberg, N. Yang, R. Verdoold, and R.P.H. Kooyman, "Immunosensing by colorimetric darkfield microscopy of individual gold nanoparticle-conjugates," Sensors and Actuators B: Chemical, vol. 150, Oct. 2010, pp. 529-536.

[94] R. Verdoold, R. Gill, F. Ungureanu, and R.P.H. Kooyman, "Femtomolar DNA detection by parallel colorimetric darkfield microscopy of functionalized gold nanoparticles," Biosensors and Bioelectronics, 2011. 


\section{Chapter 2}

\section{Au nanoparticles as sensing platforms}

Abstract: This chapter reviews the fundamental aspects of the Localized Surface Plasmon Resonance (LSPR) phenomenon and the relationship between the spectral properties of the metal nanoparticles on the local environment. We describe the possible sensing strategies employing gold nanoparticles as individual sensing elements. In addition, we discuss the distance dependence of the enhancement of the electric field and its effects on the wavelength shifts. In this chapter, we introduce the concept of parallel detection of multiple individually addressed nanoparticles to determine concentrations orders of magnitude below the inverse affinity constant. 


\section{Introduction}

Metal nanoparticles can be considered as potential candidates for refractive index sensors, similar to the thin metal layers used in the well-known planar SPR method. The physical background of such an application is the dependence of the optical extinction (absorption and scattering) on the refractive index of the environment. This has been demonstrated experimentally by monitoring the shift of the extinction maximum as a function of the refractive index of the surrounding medium[1-3]. For example, for silver particles, shifts up to $20 \mathrm{~nm}$ for a change of 0.1 in the refractive index were experimentally demonstrated [2]. Similarly, the adsorption of molecules to the nanoparticle surface should lead to a change in the refractive index around the nanoparticle, resulting in a spectral shift. First experimental data were obtained for a silver nanoparticle [4]. The authors observed a maximum spectral shift of $40 \mathrm{~nm}$ after the nanoparticle was fully covered with a layer of molecules of low molecular mass.

Furthermore, optical interaction effects are larger for plasmonic particles than those of the most commonly used optical reporters [5,6], making possible the optical detection and characterization of individual particles using standard instrumentation [7-10]. The low absolute detection limits together with the possibility of single particle detection hold the promise of extreme sensitivity [8].

As early as in 1850 Michael Faraday started scientific work on the properties of colloidal gold. In his work [11], he advanced for the first time the idea that the color of the nanoparticles is the result of the interaction of light with the tiny beads. Only a few decades later, in 1908 [12], Gustav Mie gave a full quantitative description of the scattering behavior of spherical nanoparticles, albeit without a discussion on the possibility of a collective resonant oscillation of conduction electrons, which we nowadays call surface plasmons.

This chapter focuses on determining the characteristics of Au nanoparticles as sensing platforms. We start with a discussion on the principles of Localized Surface Plasmon Resonance (LSPR). Next, applications of the LSPR effect involving gold 
$\overline{\text { nanoparticles in biosensing are detailed. In the same section, different sensing approaches }}$ will be discussed. In addition, we determine the characteristics (e.g. expected spectral shifts, sensitivity, LOD) of such sensing approaches using numerical methods.

\section{Localized Surface Plasmon Resonance}

\subsection{Quasi-static approximation}

The factors that govern the plasmon excitation for spherical particles can be identified using the simplest theoretical model, the quasi-static approximation. Although this model is not quantitatively accurate, it is a useful tool in providing a good understanding of the underlying physics of the LSPR effect.

The quasi-static approximation considers the interaction of an electromagnetic wave with a spherical particle much smaller than the wavelength of light. In these conditions, the electric field can be considered constant over the particle and the interaction is governed by electrostatics rather than electrodynamics.

We will start by considering the interaction of an electromagnetic wave, $\boldsymbol{E}_{\boldsymbol{0}}$ with a spherical nanoparticle of radius $\boldsymbol{R}$ of dielectric constant $\varepsilon$, embedded in a dielectric medium, $\boldsymbol{\varepsilon}_{\boldsymbol{m}}$, as represented in Figure $2-1$. For many practical cases, $\boldsymbol{\varepsilon}_{\boldsymbol{m}}$ is taken as a real, positive number. The $\boldsymbol{k}$ vector, considered along the $\mathrm{X}$-axis of the coordinate system, gives the propagation direction of the light.

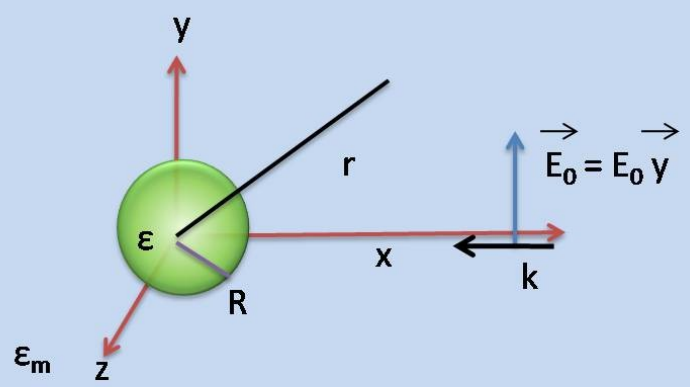

Figure 2-1 Schematic representation of a spherical particle, of radius $\mathrm{R}$ embedded in a surrounding medium, $\varepsilon_{\mathrm{m}}$, placed in a constant electric field, $\mathrm{E}_{0}$ 
A standard result from electrostatic theory is that in a dielectric sphere placed in an electric field a dipole is induced with dipole moment, $\mathrm{p}=\alpha \times \mathrm{E}$. The polarizability $\alpha$ can be determined by solving Laplace's equation for this situation as:

$$
\alpha=4 \pi \varepsilon_{0} \frac{\varepsilon-\varepsilon_{m}}{\varepsilon+2 \varepsilon_{m}} R^{3}
$$

From this equation, we can observe that the polarizability at $\varepsilon=-2 \varepsilon_{m}$ has a singularity, resulting in a very large induced dipole and thus a largely enhanced local electric field. High values for $\alpha$ can be obtained if materials with $\varepsilon<0$ are used. It turns out that for a limited spectral region this requirement is met for compounds with free conduction electrons, such as metals. For the noble metals Au and Ag, this region falls within the visible domain. Then, the condition $\varepsilon=-2 \varepsilon_{m}$ can be interpreted as a situation where the conduction electrons of the particle undergo a coherent oscillation with large amplitude, as illustrated in Figure 2-2a. This phenomenon is denoted as Local Surface Plasmon Resonance (LSPR). Figure 2-2b depicts the scattering spectrum of an individual $80 \mathrm{~nm} \mathrm{Au}$ nanoparticle. The maximum of the scattering efficiency is reached at the wavelength where $\varepsilon=-2 \varepsilon_{\mathrm{m}}$. This position is denoted as the Plasmon Peak (PP).

(a)
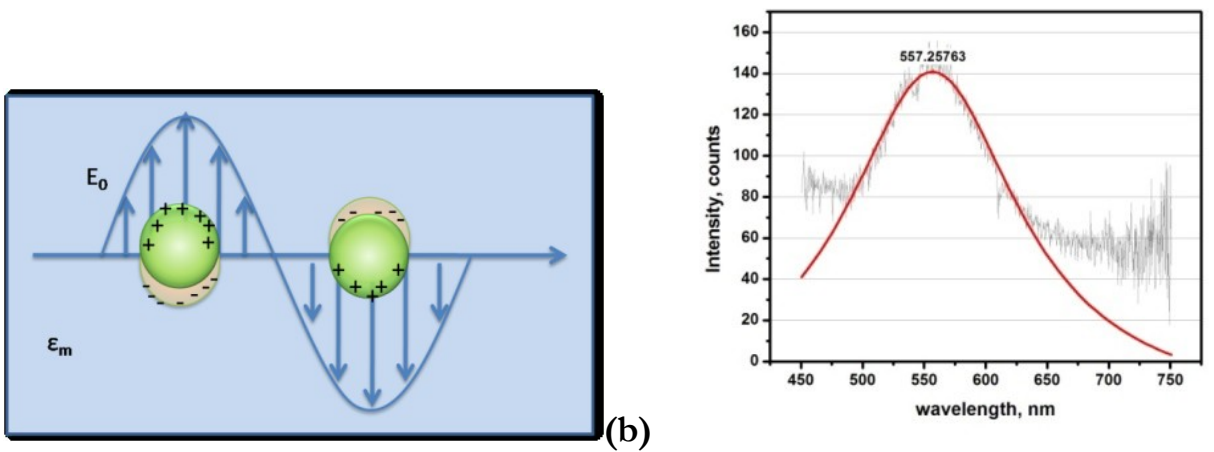

Figure 2-2 (a) Schematic representation of the LSPR effect, showing the displacement of the electronic cloud in respect to the positive nuclei (b) Typical scattering spectrum of an individual 80 $\mathrm{nm}$ Au particle immobilized on glass. The grey curve represents the raw spectra while the fitting is represented by the red curve. 
From fundamental scattering theory, we know [13] that the cross-sections for light scattering and absorption are given by:

$$
C_{a b s}=k \operatorname{Im}(\alpha){ }_{\&} C_{s c a}=\frac{k^{4}}{6 \pi \varepsilon_{0}}|\alpha|^{2}
$$

From these equations, we can conclude that a large polarizability results in a large scattering cross-section. For $\mathrm{Au}$ and $\mathrm{Ag}$ nanoparticles even single particles can be seen with the naked eye provided they are irradiated with a wavelength where $\varepsilon=-2 \varepsilon_{m}$. For example, Figure 2-3 illustrates a darkfield (DF) image where the scattering of individual Au nanoparticles can be visualized. The colour and the intensity of each spot are given by the spectral properties of the visualized nanoparticles.

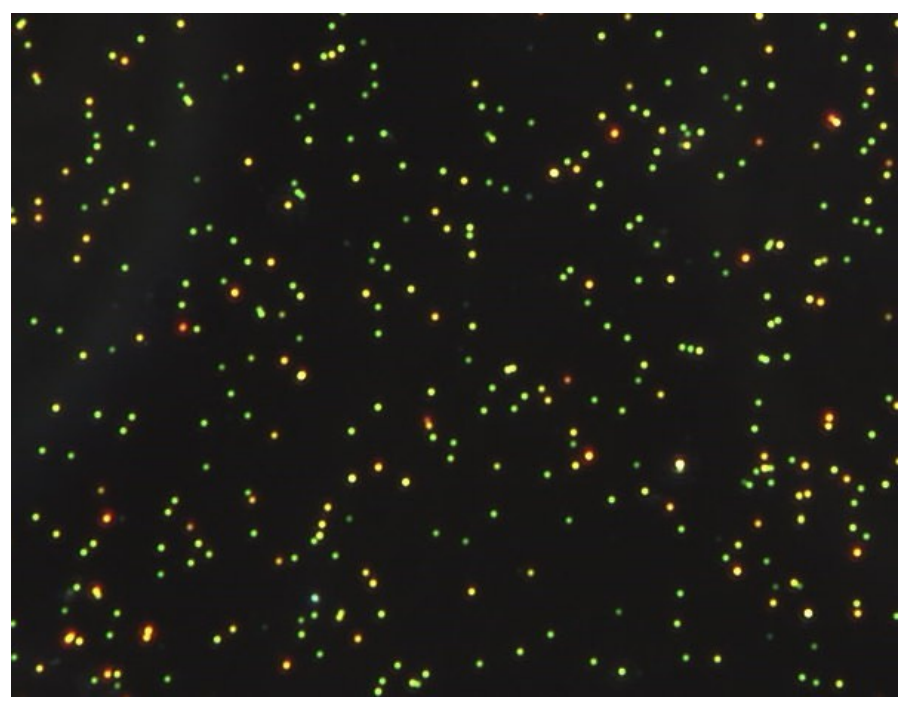

Figure 2-3 A darkfield image $\left(270 \times 165 \mu \mathrm{m}^{2}\right)$ of individual $80 \mathrm{~nm}$ Au nanoparticles immobilized on glass surface. The variations in color are given by the different spectral properties of the individual nanoparticles.

The optical properties of spherical particles much smaller than the incident wavelength can be described using the quasi-static approximation model. The increase in size negatively affects the capacity of light to polarize the nanoparticle homogeneously. This will lead to retardation effects expressed by an observable red shift of the plasmonic peak and a broadening of the plasmon band [14]. Although analytical methods are 
preferable, the introduction of numerical methods allows a higher flexibility in modelling a large variety of scenarios (e.g. different illumination conditions, peculiar shapes or sizes of nanoparticles). In the next section, a short description of the numerical method used in this work will be given.

\subsection{Discrete Dipole Approximation (DDA) method}

For the calculation of the optical properties of small aggregates of nanoparticles and different geometries, Discrete Dipole Approximation (DDA) or TMatrix algorithms are widely used [15-22]. Others prefer a more semi-empirical method like the multiple multipole method MMP, claiming a better accuracy and shorter computational times [23, 24].

Each method has its advantages and disadvantages; however, for isolated particles of arbitrary shape the DDA algorithm has proven to be a powerful tool.

In this technique, the nanoparticle is divided in a cubic lattice of polarizable elements. The major assumption of this method is that all the constituent elements of the geometry behave as dipoles, and these elements relate only through dipole-dipole interactions. Here, the structure is composed of $\mathrm{N}$ point dipoles, each with polarizability $\alpha_{i}$ centred at position $r_{i}$ with respect to a reference system. The polarization in each element induced by the local electric field $\boldsymbol{E}_{l o c}$ is:

$$
P_{i}=\propto_{i} E_{l o c}\left(r_{i}\right)
$$

with $\alpha_{i}$ the polarizability of element i. The local field of each dipole element is the sum of the incident electric field and the contribution of all other dipoles.

$$
E_{l o c}\left(r_{i}\right)=E_{i n c, j}+E_{d i p, j}=E_{0} \exp \left(i k r_{j}\right)-\sum_{j \neq i} A_{i j} \cdot P_{j}
$$

where $\mathrm{E}_{0}$ is the amplitude and $\mathrm{k}$, the wave vector of the incident wave and $\mathrm{A}$ represents the interaction matrix. The expression for the extinction cross-section is given by:

$$
\mathrm{C}_{\mathrm{ext}}=\frac{4 \pi \mathrm{k}}{\left|\mathrm{E}_{0}\right|^{2}} \sum_{\mathrm{j}=1}^{\mathrm{N}} \mathrm{E}_{\mathrm{loc}, \mathrm{j}}^{*} \cdot \mathrm{P}_{\mathrm{j}}
$$


The dielectric constants of both the metal and the surroundings are contained in the expression of the polarizabilities, $\alpha_{\mathrm{i}}$. The explicit formula was developed by Draine and Goodman [25], such that the dipole lattice of an infinite metal reproduces the continuum dielectric response of the solid to the electromagnetic radiation. Although not exact, the theory developed by them gives results within 10\% accuracy in the localization of the plasmon peak for particles of arbitrary shape and composition [20].

\section{Gold nanoparticles as sensing elements}

As seen from equation 2.1 any change (local or global) in the refractive index of the medium in which the nanoparticles are immersed will alter the resonance conditions leading to a shift in the position of the PP. By extension, the coating of the nanoparticle with a dielectric shell (e.g. proteins, DNA) should give rise to an observable change in their optical properties. This implies that metal nanoparticles can be used as refractive index sensors. For practical reasons we will limit our discussion to applications of $\mathrm{Au}$ nanoparticles as sensors.

For Au nanoparticles to be considered as sensing elements, several criteria have to be fulfilled. First, on top of the nanoparticle, a layer of receptor molecules (e.g. antibody, DNA molecule), able to bind specifically to the 'target' molecules of interest, is required. When target molecules are added to the system, they will bind to the receptor molecules of the nanoparticles. The binding of analytes to the receptors will replace a certain volume of water. The refractive index difference between the water molecules and analyte causes an alteration in the local refractive index around the nanoparticle. This change will lead to an adjustment in the resonance conditions of the nanoparticle resulting in a shift of the PP.

In addition, the ability to detect small changes in the extinction spectrum due to molecular interactions ensures the successful utilization of Au nanoparticles (GNPs) as sensing elements. Furthermore, functionalization strategies are necessary for the proper performance of biomolecules and implicitly a working GNP sensor. The immobilization 
of the nanoparticles on a transparent surface gives the additional advantage to detect single GNPs.

With the appropriate experimental arrangements for detection, the interaction between the receptor molecules immobilized on the GNP and the target molecules can be investigated. Binding reactions can be studied in a number of ways using individual GNPs as sensors; two of them are schematically depicted in Figure 2-4.

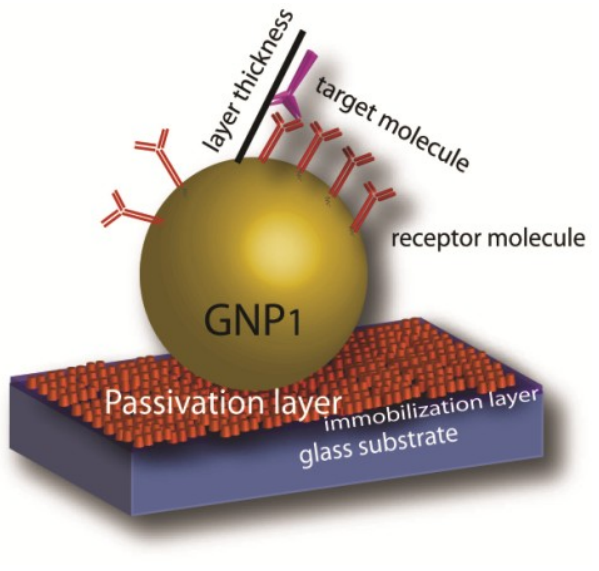

(a)

Figure 2-4 Schematic representation of the sensing principle using gold nanoparticle as sensing element. (a) Non-amplified assay where the target interacts with the receptor molecules immobilized on the surface of the gold nanoparticle. (b) Amplified direct assay; the target molecules, linked on the surface of the secondary particles react with the receptor molecules on the immobilized nanoparticle bringing the two nanoparticles in close proximity.

One detection approach is to monitor the position of the PP of individual GNPs when the receptor molecules, attached on the particle's surface, interact with the target molecules in solution (Figure 2-4a). The binding events produce a change in the local refractive index around the nanoparticle resulting in a noticeable spectral shift. Eventually, the response slows to reach a new equilibrium value. If sufficient target molecules are added in solution, the final position of the PP can be associated with a full coverage of the nanoparticle. By parallelization, that is, the simultaneous measurement of the signal of a collection of individual functionalized nanoparticles, a good sensitivity can be obtained 
due to the high number of available receptor molecules. Yet, not all receptors are necessarily available for interaction due to inactivity or in correct orientation leading to steric hindrance. Therefore, this method has limitations that affect the overall sensitivity of the method.

Another detection approach is to use an amplification step (Figure 2-4b). Here, a second functionalized nanoparticle is used. The binding reaction between the partner molecules brings the two particles in close proximity inducing a strong PP shift for each interaction. As we will see later in this chapter, the detection sensitivity of this method should be higher than the non-amplified approach.

Altogether, these arguments propose gold nanoparticles as viable candidates for sensing platforms. Next in this chapter, we will discuss the properties required for the gold nanoparticles to be used as sensing elements, in two sensing approaches: single particle sensing and coupled particle sensing.

\section{Single nanoparticle sensing}

In this section, we will investigate the optical properties of gold nanoparticles using DDA software and find the attributes of individual GNPs to achieve optimum sensing abilities. First, we describe the optical properties of gold nanoparticles covered by a layer of biological molecules.

For the study of a GNP covered by a layer of biomolecules, the generated geometry is a two layered sphere embedded in a dielectric medium, $\boldsymbol{\varepsilon}_{\mathrm{w}}$, as schematically represented in Figure 2-5a. The nanoparticle with dielectric function $\varepsilon(\omega)$ with radius $\boldsymbol{R}$ is surrounded by a biomolecular shell of thickness $\boldsymbol{d}$ and refractive index $\boldsymbol{n}_{p}{ }^{2}$ For the simulations, the dielectric function of the nanoparticle was adopted from Johnson and Christy [26]. The available data in literature show that, for proteins, a refractive index ranging from 1.4- 1.7 can be used [27]. Here, the refractive index of the shell was taken as $\mathbf{n}_{\mathrm{p}}=1.5$. The system was embedded in water, with refractive index of 1.33 . For such a ${ }^{2}$ Note that, generally $\varepsilon$ and $\mathrm{n}$ are related by $\varepsilon=\mathrm{n}^{2}$ 
system, the localization of the plasmon peak was calculated as a function of several variables.

As we are interested in monitoring the binding of biomolecules and biomolecular pairs with layer thicknesses up to $20 \mathrm{~nm}$ it is important that the induced dipole field is of sizable amplitude in that range. The extension of this field was determined by monitoring the shift in the position of the plasmon peak (PP) as a function of the shell thickness for different particle sizes. The shell represents the biomolecular layer(s) bound to the nanoparticle, as schematically represented in Figure 2-5a. The practical importance of the obtained results (Figure 2-5b) is that it gives an impression of the dynamic range. This is defined as the range in $\mathrm{nm}$ where the nanoparticle can still feel changes in the refractive index of the local medium. Depending on the size of the nanoparticles, the dynamic range varies (Figure 2-5b); smaller particles have a more confined field than larger particles, making them usable for the detection of low molecular mass molecules. For shell diameters ranging from 6 to $20 \mathrm{~nm}$, depicted as a yellow region in Figure 2-5b, 60 and 80 $\mathrm{nm} \mathrm{Au}$ particles are preferred. Owing to these small dynamic ranges $(\sim$ tens of nanometers) Au nanoparticles are less sensitive to external factors (e.g. temperature) than conventional SPR systems, which have larger confinement regions ( $\sim$ hundreds of nanometers) [28].

(a)
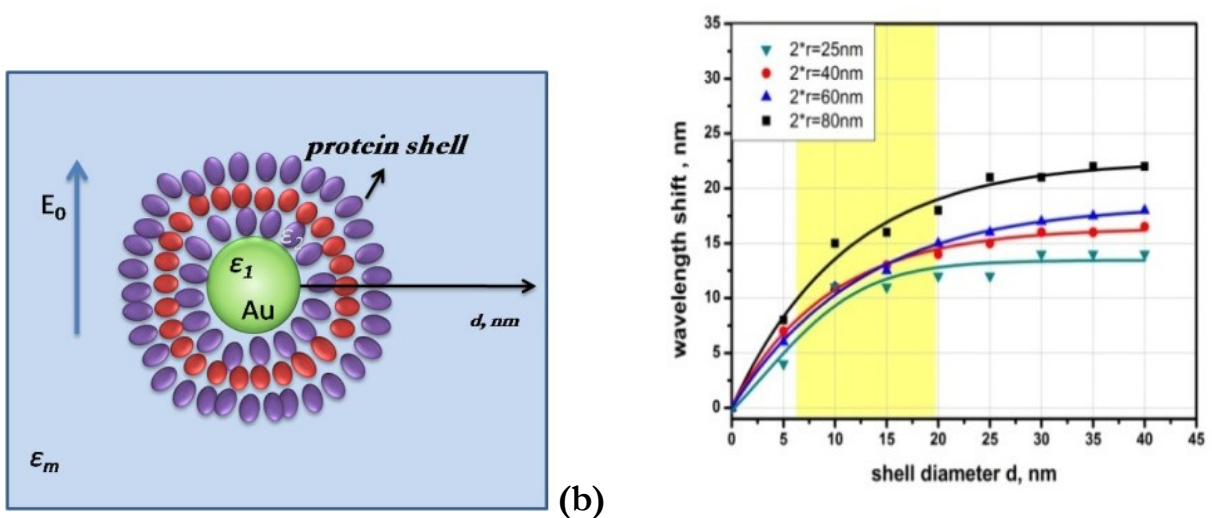

Figure 2-5 (a) A schematic representation of the layered model used in our simulations. The Au nanoparticle of radius $\mathrm{R}$, covered by a shell of protein of thickness $\mathrm{d}$, is embedded in water, with a refractive index, $\mathrm{n}_{\mathrm{w}}=1.33$. (b) The results of the extension of the field for various particle sizes. 
A critical parameter for the visualization of the nanoparticles is the scattering efficiency of the nanoparticle, which is proportional with $\mathrm{R}^{6}$ of the particle (cf. equation 2.2). From this perspective, larger particles are preferable.

Summarizing, the optimum characteristics of a nanoparticle as a sensing element are:

- Large scattering efficiency; this points toward the use of relatively large nanoparticles .

- Limited dynamic range (the distance range where the nanoparticles still feels changes in the local refractive index) of molecular interactions $(5-30 \mathrm{~nm})$.

Therefore, a good compromise is using gold particles of 60 or $80 \mathrm{~nm}$ in diameter.

A particular case of interest is monitoring the adsorption of a monolayer of biomolecules to the surface of a single nanoparticle. Let us consider a nanoparticle of radius $\boldsymbol{R}$, which is covered with an imaginary layer of water, with refractive index, $\boldsymbol{n}_{\boldsymbol{w}}$. The thickness of this layer is taken to have the diameter of the biomolecule under consideration. When a molecule, with refractive index $\boldsymbol{n}_{p}$, adsorbs to the surface of the nanoparticle it replaces an equal volume of water. The resulting effective local refractive

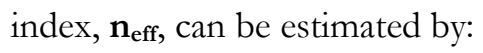

$$
n_{e f f}=n_{w}+\frac{N_{p}}{N_{t}}\left(n_{p}-n_{w}\right)
$$

Where $\boldsymbol{N}_{p}$ represents the number of biomolecules that are adsorbed on the nanoparticle's surface and $\boldsymbol{N}_{t}$ is the total number of biomolecules that can be accommodated on the particle. The total number of available binding sites, $\boldsymbol{N}_{t}$, can be estimated by calculating the surface area of the nanoparticle and comparing this with the projected area of one biomolecule. For example, on the surface of a $60 \mathrm{~nm}$ GNP we can accommodate a maximum of 400 molecules, each of6 nm diameter.

The results illustrated in Figure 2-6 show that the adsorption of a full layer of biomolecules, with $6 \mathrm{~nm}$ in diameter, on the surface of the nanoparticle $(60 \mathrm{~nm})$ would give a $7 \mathrm{~nm}$ shift, which can be easily recorded by a spectrometer capable of measuring spectra of individual nanoparticles. For a standard spectrometer, which is able to detect 1 
$\overline{\mathrm{nm} \text { shift, we expect that the adsorption of } \sim 50 \text { biomolecules ( } 6 \mathrm{~nm} \text { diameter }) \text { per particle }}$ can be detected. If low mass molecules are adsorbed on the surface of the nanoparticle, the detection limit deteriorates in view of the thinner layer formed by smaller molecules.

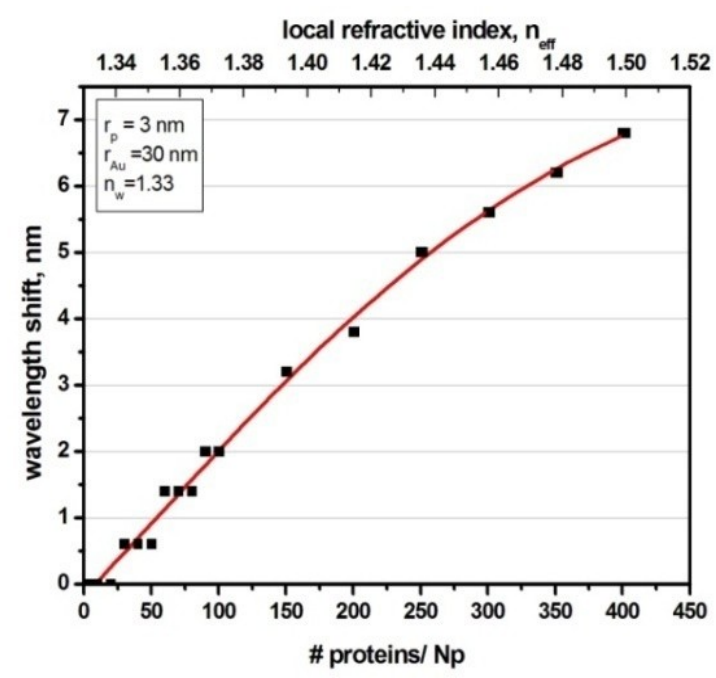

Figure 2-6 The spectral response of a $60 \mathrm{~nm}$ Au particle, embedded in water, to an increasing number of adsorbed $6 \mathrm{~nm}$ diameter biomolecules.

Although these absolute theoretical detection limits seem quite impressive it should be noted that in many practical sensor applications the main parameter of interest is the minimum concentration that can be detected. This implies that in this type of applications, nanoparticles will only be useful if a large number of identically prepared particles can be simultaneously and individually detected.

In order to make an estimate of the limit of detection (LOD) of such a sensing approach, with multiple individual nanoparticles, we developed a Monte Carlo simulation adapted to our approach.

Let us assume a concentration $\boldsymbol{C}_{\boldsymbol{x}}$ of analyte $\boldsymbol{X}$, characterized by an affinity constant $\boldsymbol{K}_{\mathbf{x}}$. Assuming that the binding process of that analyte shows a Langmuir behavior, we can estimate the net receptor coverage, $\Gamma$, being [29]:

$$
\Gamma=\frac{K_{x} C_{x}}{1+K_{x} C_{x}}=\frac{N_{\text {bound }}}{N_{t}}
$$


Next, in our Monte Carlo procedure the analyte molecules are randomly placed over all the available binding sites. This process is repeated until $\Gamma$, dictated by the reaction characteristics, is reached. The result of this program gives the distribution of particles with a certain covered fraction. From this distribution, the fraction of $\mathrm{Au}$ nanoparticles that have bound the minimum detectable binding sites is determined, and from this, the fraction of bound particles is calculated.

As an example, we assume the following:

- $3000 \mathrm{Au}$ nanoparticles with $60 \mathrm{~nm}$ diameter, each with 400 available binding sites;

- The presence in solution of an analyte with a binding affinity $K_{x}=10^{7} \mathrm{M}^{-1}(\mathrm{a}$ typical value for protein immuno-bindings) [30];

- 50 binding events is the determined minimum detection limit for a $60 \mathrm{~nm} \mathrm{Au}$ particle (Figure 2-6).

For this system, we applied our Monte Carlo procedure and from this, we calculated the number of $\mathrm{Au}$ nanoparticles that have bound the minimum detectable binding sites and determined the binding fraction for different analyte concentrations. Figure $2-7 \mathrm{a}$ shows an example of the nanoparticle distribution for $C_{x}=2 * 10^{-7} \mathrm{M}$. The yellow region represents the selection of those particles, $\boldsymbol{N}_{\text {bound }}$, that have at least 50 occupied binding sites.

(a)

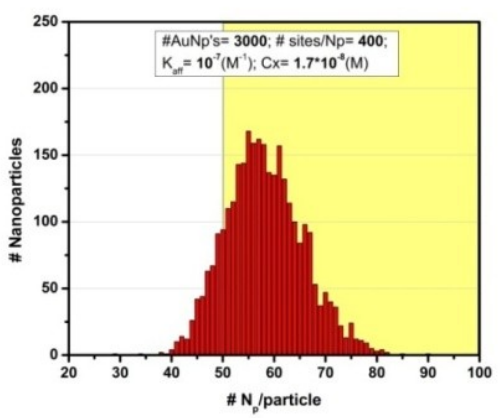

Figure 2-7 (a) The distribution of the Au nanoparticles with a certain occupation rate for $C_{x}=$ $1.7 * 10^{-8} \mathrm{M}$. (b) The theoretical concentration dependence

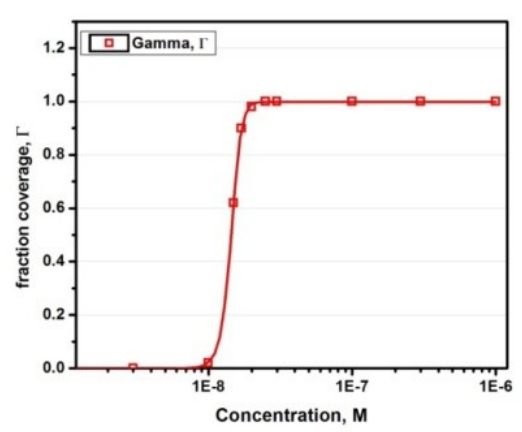

(b) 
The results depicted in Figure 2-7b indicate that for such a system the LOD is situated in the range of tens of nanomolar, similar or even worse compared to that of conventional SPR sensor systems [31]. This limit can be somewhat lower if smaller particles are used or larger biomolecules. However, by using an amplification step, which enables the detection of single binding events, the detection limit can be improved by several orders of magnitude and this approach is discussed in the following section.

\section{Coupled nanoparticle sensing}

One strategy to lower the limits of detection is to use an amplification approach. One way to accomplish this is to bring one functionalized particle in close proximity to a second functionalized particle through the specific interaction of the partner molecules. In the next section, we will explain the reasons why this leads to a strong change in the resonance conditions.

\subsection{Quasi-static approach}

In order to get physical insight into the amplification process, we will give an analytical description of the phenomena using the quasi-static approximation.

Consider two gold nanospheres with radii $\boldsymbol{R}_{\boldsymbol{1}}$ and $\boldsymbol{R}_{\boldsymbol{Z}}$ and dielectric permittivity $\boldsymbol{\varepsilon}$ $1(\omega), \varepsilon_{2}(\omega)$, respectively, embedded in a medium with dielectric permittivity $\varepsilon_{m}$, as represented in Figure 2-8. The distance $\boldsymbol{d}$ separates the two particles (surface to surface) from each other. Let us consider that the external electric field, $\boldsymbol{E}_{\boldsymbol{0}}$, applied to the system is perpendicular to the axis connecting the centres of the two nanoparticles. The local electric field of one particle is influenced by the induced dipole moment of the second particle and vice versa. 


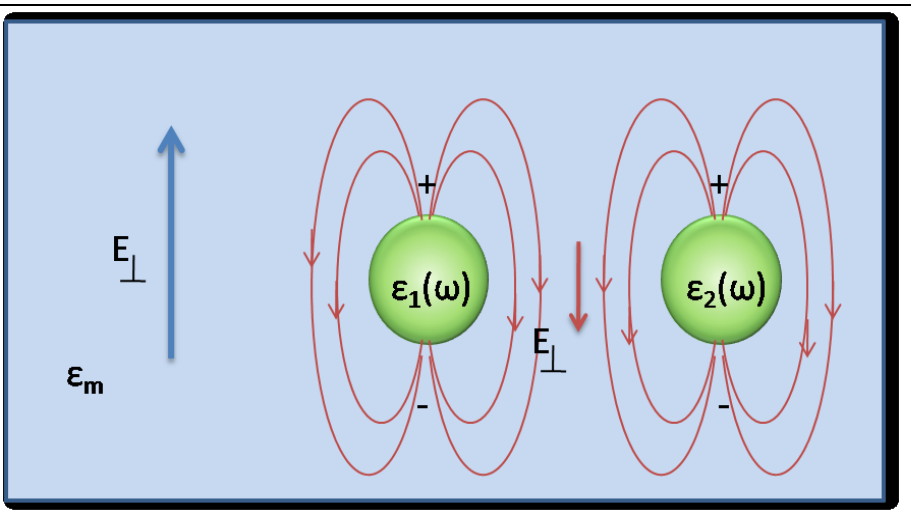

Figure 2-8 Schematic representation of the behavior of two closely located particles placed in an electric field perpendicular to the system axis

Thus, the local electric field acting on each particle is the sum of the external electrical field and the induced local electric field as follows:

$$
\begin{aligned}
& E_{\perp, 1}^{l o c}=E_{0 \perp}-\frac{p_{2}}{4 \pi \varepsilon_{0} \varepsilon_{m} d^{3}} \\
& E_{\perp, 2}^{l o c}=E_{0 \perp}-\frac{p_{1}}{4 \pi \varepsilon_{0} \varepsilon_{m} d^{3}}
\end{aligned}
$$

In order to find the effective polarizability associated with the system, we substitute the expression for the dipole moments in the above equations:

$$
p_{1}=\alpha_{1} E_{\perp, 1}^{l o c} \& p_{2}=\alpha_{2} E_{\perp, 2}^{l o c}
$$

Thus, we get the expression of the local electric field of the first nanoparticle:

$$
E_{\perp, 1}^{l o c}=\frac{1-\frac{\alpha_{2}}{4 \pi \varepsilon_{0} \varepsilon_{m} d^{3}}}{1-\frac{\alpha_{1}}{4 \pi \varepsilon_{0} \varepsilon_{m} d^{3}}} E_{\perp, 2}^{l o c}
$$

By substituting Eq 2.11 into equations 2.8 and 2.9, we get the effective polarizability of the first particle: 


$$
\alpha_{\perp, 2}^{e f f}=\alpha_{2} \frac{1-\frac{\alpha_{1}}{4 \pi \varepsilon_{0} \varepsilon_{m} d^{3}}}{1-\frac{\alpha_{1} \alpha_{2}}{\left(4 \pi \varepsilon_{0} \varepsilon_{m} d^{3}\right)^{2}}} \& \alpha_{\perp, 1}^{e f f}=\alpha_{1} \frac{1-\frac{\alpha_{2}}{4 \pi \varepsilon_{0} \varepsilon_{m} d^{3}}}{1-\frac{\alpha_{1} \alpha_{2}}{\left(4 \pi \varepsilon_{0} \varepsilon_{m} d^{3}\right)^{2}}}
$$

The overall effective polarizability of the entire system is:

$$
\alpha_{\perp}^{e f f}=\alpha_{\perp, 1}^{e f f}+\alpha_{\perp, 2}^{e f f}=\frac{\left(\alpha_{1}+\alpha_{2}\right)-\frac{\alpha_{1} \alpha_{2}}{2 \pi \varepsilon_{0} \varepsilon_{m} d^{3}}}{1-\frac{\alpha_{1} \alpha_{2}}{\left(4 \pi \varepsilon_{0} \varepsilon_{m} d^{3}\right)^{2}}}
$$

In the case of a parallel external electric field, the local electric fields are:

$$
\begin{aligned}
& E_{\|, 1}^{l o c}=E_{0 \|}+\frac{p_{2}}{4 \pi \varepsilon_{0} \varepsilon_{m} d^{3}} \\
& E_{\|, 2}^{l o c}=E_{0 \|}+\frac{p_{1}}{4 \pi \varepsilon_{0} \varepsilon_{m} d^{3}}
\end{aligned}
$$

In the same manner, we make the substitution of the dipole moments in the expressions of the electric fields:

$$
E_{\|, 1}^{l o c}=\frac{1+\frac{\alpha_{2}}{4 \pi \varepsilon_{0} \varepsilon_{m} d^{3}}}{1+\frac{\alpha_{1}}{4 \pi \varepsilon_{0} \varepsilon_{m} d^{3}}} E_{\|, 2}^{l o c}
$$

resulting in the effective polarizabilities for each particle:

$$
\alpha_{\|, 2}^{e f f}=\alpha_{2} \frac{1+\frac{\alpha_{1}}{4 \pi \varepsilon_{0} \varepsilon_{m} d^{3}}}{1-\frac{\alpha_{1} \alpha_{2}}{\left(4 \pi \varepsilon_{0} \varepsilon_{m} d^{3}\right)^{2}}} \& \alpha_{\|, 1}^{e f f}=\alpha_{1} \frac{1+\frac{\alpha_{2}}{4 \pi \varepsilon_{0} \varepsilon_{m} d^{3}}}{1-\frac{\alpha_{1} \alpha_{2}}{\left(4 \pi \varepsilon_{0} \varepsilon_{m} d^{3}\right)^{2}}}
$$

The effective polarizability of the two particles, is given by:

$$
\alpha_{\|}^{e f f}=\alpha_{\|, 1}^{e f f}+\alpha_{\|, 2}^{e f f}=\frac{\left(\alpha_{1}+\alpha_{2}\right)+\frac{\alpha_{1} \alpha_{2}}{2 \pi \varepsilon_{0} \varepsilon_{m} d^{3}}}{1-\frac{\alpha_{1} \alpha_{2}}{\left(4 \pi \varepsilon_{0} \varepsilon_{m} d^{3}\right)^{2}}}
$$


If the two particles are located far enough from each other, they will not interact and the effective polarizability of each particle is:

$$
\alpha_{\|}^{e f f}=\alpha_{1}=\alpha_{2}
$$

For practical reasons we assume both particles to have the same dielectric function. To understand what happens with the particle system we study it at resonance. In this condition, the polarizability of the system is a maximum, thus the denominator $1-$ $\frac{\alpha_{1} \alpha_{2}}{\left(4 \pi \varepsilon_{0} \varepsilon_{m} d^{3}\right)^{2}} \rightarrow 0$.By replacing in this relation the expressions of the polarizability, we get the following relation:

$$
\left(4 \pi \varepsilon_{0}\right)^{2}\left(\frac{\varepsilon-\varepsilon_{m}}{\varepsilon+2 \varepsilon_{m}}\right)^{2} R_{1}^{3} R_{2}^{3}= \pm\left(4 \pi \varepsilon_{0} \varepsilon_{m}\right)^{2}\left(d^{2}\right)^{3}
$$

Thus:

$$
\left(\varepsilon-\varepsilon_{\mathrm{m}}\right)= \pm\left(\varepsilon+2 \varepsilon_{\mathrm{m}}\right) \varepsilon_{\mathrm{m}}\left(\frac{d^{2}}{R_{1} R_{2}}\right)^{3 / 2}
$$

From this expression, we find that for the perpendicular polarization the resonance condition is:

$$
\varepsilon_{\perp}=\frac{1-2 \varepsilon_{m}\left(\frac{d^{2}}{R_{1} R_{2}}\right)^{3 / 2}}{1+\varepsilon_{m}\left(\frac{d^{2}}{R_{1} R_{2}}\right)^{3 / 2}} \varepsilon_{m}
$$

For the parallel polarization the resonance condition is:

$$
\varepsilon_{\|}=\frac{1+2 \varepsilon_{m}\left(\frac{d^{2}}{R_{1} R_{2}}\right)^{3 / 2}}{1-\varepsilon_{m}\left(\frac{d^{2}}{R_{1} R_{2}}\right)^{3 / 2}} \varepsilon_{m}
$$


Equations $2.22 \& 2.23$ show that, for the two polarization states, the dielectric function of the nanoparticle system evolves in opposite directions. Implicitly, a red or a blue shift in the PP position will result. If the wavelength dependence of the dielectric function is known, the corresponding PP position can be predicted.

\subsection{Numerical results}

As already mentioned, the quasi-static approach provides a good physical insight into the LSPR optical phenomena and its effects. However, for a more accurate quantitative description of the optical properties of coupled $\mathrm{Au}$ nanoparticle systems, relevant to us, we have to rely on numerical methods. To this end, we have used the DDA algorithm. The input parameters that define the studied system are the shape, the effective radius, the dielectric function of the material, and the refractive index of the embedding medium. The geometry is defined by the effective radius, representing the radius of the equivalent sphere having the volume equal with the volume of the object. For the dielectric function of the material, the tabulated values from Johnson and Christy [26] were used. The entire system was embedded in water with a refractive index of 1.33. For the simulations, we considered a particle $\boldsymbol{p}_{\boldsymbol{1}}$, with radius $\boldsymbol{R}_{1}$ and dielectric permittivity $\boldsymbol{\varepsilon}_{1}(\boldsymbol{\omega})$ in the centre of the coordinate system. The second particle, with radius $\boldsymbol{R}_{2}$ and dielectric permittivity $\varepsilon_{2}(\omega)$, is placed at a certain distance $\boldsymbol{d}$ (surface to surface) from the first particle. We have simulated the system in unpolarized light and in polarized light (perpendicular and parallel) as depicted in Figure 2-9.

For the situation where the light is un-polarized (Figure 2-9a), we perform the following actions:

- The $\mathrm{k}$ vector is along the $\mathrm{X}$-axis;

- The electric field is directed along the Y axis;

- The axis of the particle pair system is rotated around the $\mathrm{k}$ vector $\left(360^{\circ}\right.$ with a $3.6^{0}$ angle resolution) and a rotational averaging is performed. 

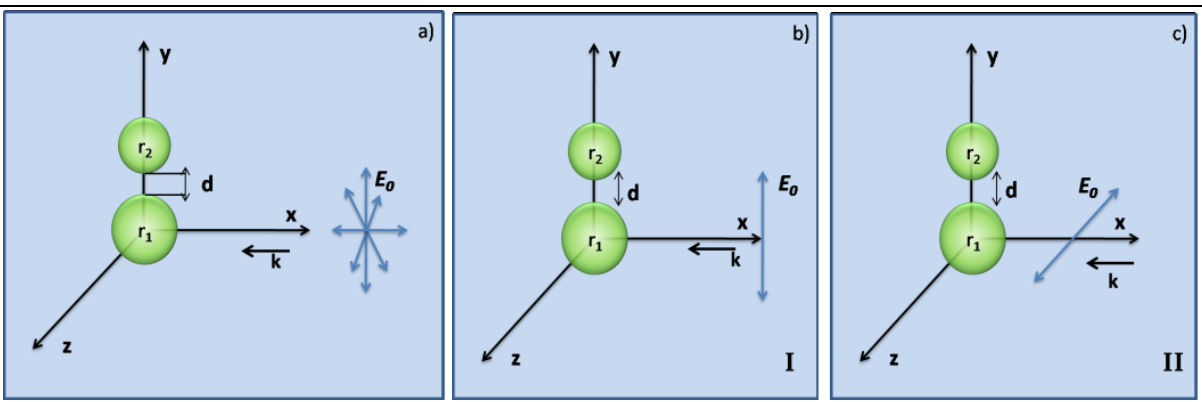

Figure 2-9 Schematic representation of the particle system in respect to the polarization of light: (a) un-polarized, (b) parallel with the system axis and (c) perpendicular. The distance $d$ is defined as the surface-to-surface inter-particle distance. The light propagation is along the $\mathrm{X}$-axis.

(a)

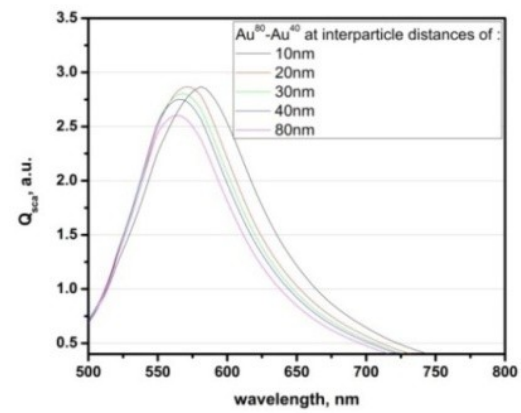

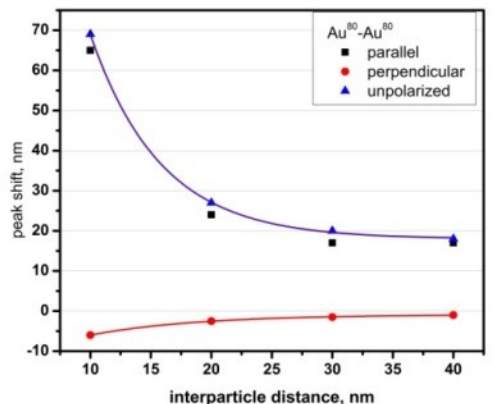

(b)

Figure 2-10 The numerical result in the case of two-particle system for different polarizations. (a) The scattering spectra for the $\mathrm{Au}^{80}-\mathrm{Au}^{40}$ particle system at different inter-particle distances; (b) The expected wavelength shifts for different polarizations states of the incident light

First, we investigated the dependence of the PP position on the interparticle distance. As previously mentioned, we intend to visualize the particles in a dark field setup for experimental validation of the model; thus, in our simulations we considered $80 \mathrm{~nm} \mathrm{Au}$ particles as the primary particle and a $40 \mathrm{~nm}$ Au particle as the added particle. The resulting simulated spectra are shown in Figure 2-10a. By decreasing the inter-particle distance, we observe a strong red shift of the position of the PP. This effect is due to the strong dipolar interaction that occurs between the two nanoparticles. Figure $2-10 \mathrm{~b}$ shows the numerical results for the $\mathrm{Au}^{80}-\mathrm{Au}^{40}$ pair in different polarization states. For un-polarized and parallel polarization, we observe a strong red shift of the 
$\overline{\text { plasmon peak at shorter distances. For the perpendicular polarization, a moderate blue }}$ shift is observed. These results are in good agreement with the available experimental results from literature [32-34].

Another point of interest is to find the optimal characteristics of the particle pair for sensing purposes. To this end, the PP shift was calculated for different pairs of particles and several inter-particle distances. We search to find the optimum pair that gives the highest peak shift at 10-30 nm inter-particle distances (the size of the molecules involved in the binding event). For the calculations, we considered an $80 \mathrm{~nm}$ particle in the centre of the coordinate system, $\boldsymbol{p}_{1}$, and one secondary particle $\boldsymbol{p}_{\boldsymbol{2}}$ placed at distance $\boldsymbol{d}$ (surface to surface) from the first particle in un-polarized light.

First, the distance $\boldsymbol{d}$ was fixed, and we varied the size of the secondary particle and calculated the peak shift for these conditions (Figure 2-11a). These simulations show a stronger red shift of the plasmonic peak with increasing size of the secondary particles. Pairs with the second particle's diameter smaller than $40 \mathrm{~nm}$ show relatively small PP shifts $(<5 \mathrm{~nm})$, and we decided to discard these combinations in further calculations.

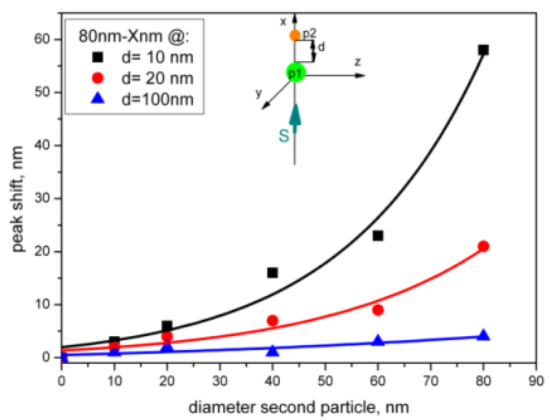

(a)

(b)

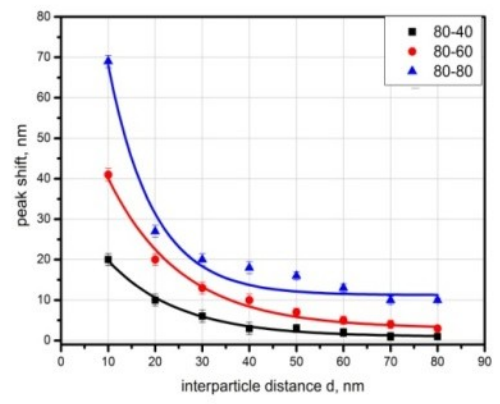

Figure 2-11 (a) The dependence of the plasmon peak on the size of the secondary particle for a $10 \mathrm{~nm}$ (square), $20 \mathrm{~nm}$ (circles) and 100nm inter-particle distance. (b) The theoretical plasmon peak shifts for different coupled nanoparticle systems obtained for various inter-particle distances. The obtained results are for a particle pair in un-polarized light.

Subsequently, we monitored the PP shift of the pair for a range of interparticle distances, for three sets $\mathrm{Au}^{80}-\mathrm{Au}^{40}, \mathrm{Au}^{80}-\mathrm{Au}^{60}$ and $\mathrm{Au}^{80}-\mathrm{Au}^{80}$ (Figure 2-11b). These 
$\overline{\text { simulations demonstrate that, in the } 10-30 \mathrm{~nm} \text { range, all three pair sets show strong red }}$ shifts of the PP upon one binding event. In a DF setup, the scattering intensity of the added particles should be low (cf. Chapter 4). Thus, the $\mathrm{Au}^{80}-\mathrm{Au}^{40}$ pair is a good compromise; for inter-particle distances between 10-30 nm we expect reasonable large PP shifts $(>5 \mathrm{~nm})$ per binding event and when the $\mathrm{Au}^{40}$ particles are introduced in a DF setup, we expect a low additional scattering intensity.

When we simulated the PP shifts for the $\mathrm{Au}^{80}-\mathrm{Au}^{40}$ pair, we considered one specific case: the particle-pair axis was set in a perpendicular or parallel orientation to the surface plane and the light was considered un-polarized. In real situations, however, the secondary functionalized particles sit where there are available binding places. Thus, the immediate question is what is the range of peak shifts to be expected for this case?

It is well known that the PP shift depends on many parameters: light polarization, particle sizes, inter-particle distance, the position of the secondary particle and number of secondary particles. Here, the pair system is studied in un-polarized light. It is well known that solutions of nanoparticles have a certain degree of size polydispersivity [35]. For simplicity, we assume this parameter to be constant. The interparticle distance is dictated by the size of the biomolecules involved in the assay. However, these molecules have a certain degree of flexibility, which leads to a change in the distance between the nanoparticles. We observed no PP shifts if the inter-particle distance was changed with $\pm 5 \mathrm{~nm}$ (data not shown). Thus, we can afford a molecular diameter change of $\pm 5 \mathrm{~nm}$. In view of these results, the inter-particle distance can be taken as constant. We used a fixed propagation direction of light along the $\mathrm{X}$-axis.

The remaining free variable is the position of the secondary particle. For these calculations, we limit ourselves to the $\mathrm{Au}^{80}-\mathrm{Au}^{40}$ particle pair for reasons given above. The position of the secondary particle was changed at different angle values in the XY plane (15 degrees resolution, $\left(0-90^{\circ}\right)$ ), as shown in Figure 2-12a, keeping the inter-particle distance constant at $20 \mathrm{~nm}$. Figure $2-12 \mathrm{~b}$ shows the expected peak shift range for different positions of the secondary particle. The theoretical results depicted in Figure 2$12 \mathrm{~b}$ show that for various positions of the secondary particle we expect peak shifts between 5 and $18 \mathrm{~nm}$. 


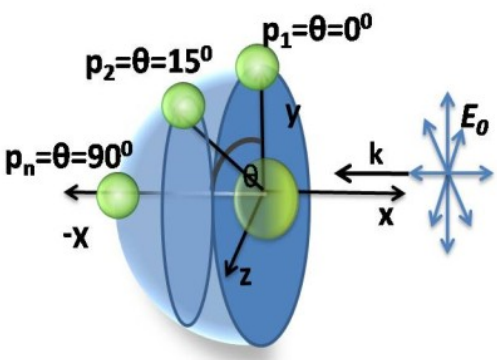

(a)

Figure 2-12 (a) An illustration representing the position of the added particles in respect to the 80 $\mathrm{nm}$ Au particle (b) The expected wavelength shift range when the position of the secondary particle is varied.

Another important practical aspect is to find out what number of added $\mathrm{Au}^{40}$ particles lead to saturation of the PP shifts. It is obvious that one $\mathrm{Au}^{80}$ can accommodate several $\mathrm{Au}^{40}$ particles. To get an impression on the overall PP shift after adding a number of $\mathrm{Au}^{40}$ particles, we monitored in a simulation the PP shift of the resulted aggregate after adding, one by one, $40 \mathrm{~nm}$ particles.

(a)

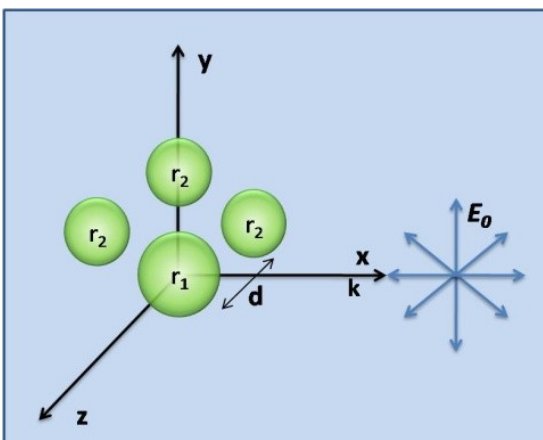

(b)

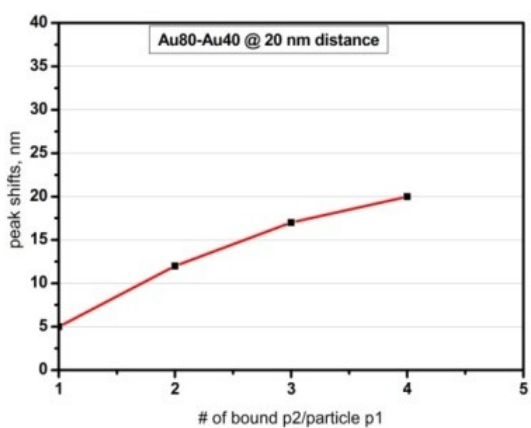

(b) The expected spectral shift in an $\mathrm{Au}^{80}-\mathrm{Au}^{40}$ system at $20 \mathrm{~nm}$ apart when multiple binding events are expected. The system was considered in un-polarized light. The purpose of the red line is to guide the eye.

Figure 2-13a depicts one of the many possible configurations and the results shown in Figure 2-13b are meant as a typical example. By examining the data presented in 
Figure 2-13b, we observe that starting with the addition of the third particle, the increase in the peak shifts becomes smaller suggesting the start of a saturation plateau.

We discussed the optical properties of the coupled nanoparticles and the factors that affect the enhancement of the peak shifts. We observed that the smallest peak shift at one binding event for an $\mathrm{Au}^{80}-\mathrm{Au}^{40}$ pair is $5 \mathrm{~nm}$, which can be easily detected with our experimental setup (details in Chapter 4).

As in the single nanoparticle sensing approach, we are interested in the limits of detection. We apply the same Monte Carlo algorithm, which was previously described in section 3. In this case we count all the particles of which at least one binding site was occupied and determine the bound fraction for different analyte concentrations.

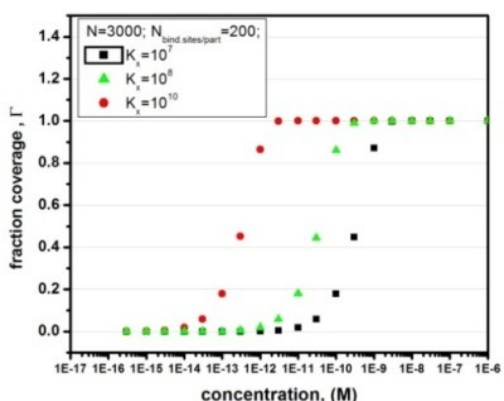

(a)

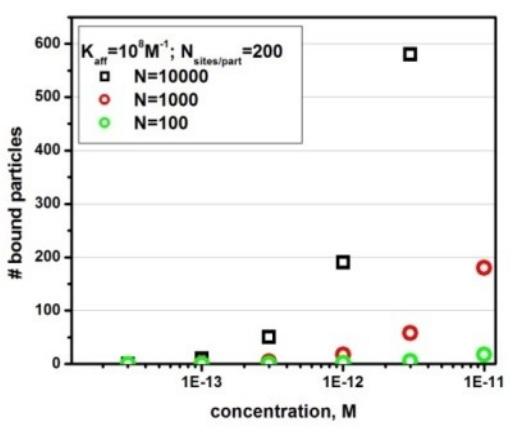

(b)

Figure 2-14 The theoretical limits of detection for an $\mathrm{Au}^{80}-\mathrm{Au}^{40}$ pair in an assay where the affinity constant of the analyte (a) or the number of available particles (b) is changed.

We presume that 3000 of $80 \mathrm{~nm}$ Au particles, each with 200 available binding sites, can be detected simultaneously. In addition, for a typical protein we assume a binding affinity $\mathrm{K}_{\mathrm{x}}=10^{7} \mathrm{M}^{-1}$. The target, with concentration $\boldsymbol{C}_{\boldsymbol{x}}$, is added. Until here, the process is the same as in the single nanoparticle sensing approach. In order to detect single binding events, we need a second set of particles, $\mathrm{Au}^{40}$ each with 75 available receptor sites, which will interact with the bound analytes. Based on geometrical considerations we found out that on a $40 \mathrm{~nm}$ Au nanoparticle could accommodate 100 molecules, with $6 \mathrm{~nm}$ in diameter. The reaction takes place until a new equilibrium state is reached. By maintaining a fixed concentration of the secondary particles $\left(10^{-10} \mathrm{M}\right)$, the 
covered fraction in this second binding process will be constant for the entire target concentration range. In addition, the fact that one $\mathrm{Au}^{40}$ particle contains many receptors introduces multivalency, resulting in a largely increased affinity constant for this second binding process. This implies that the calculated $\Gamma$ corresponds to the first binding process. The simulated results for this case are displayed on Figure 2-14a (black squares).

According to our Monte Carlo result illustrated in Figure 2-14a (black squares) we observe that the limit of detection, defined as the minimum detectable concentration for the system under consideration, lies in the pico-molar range $\left(10^{-12} \mathrm{M}\right)$. The limits of detection are lower for molecules with higher affinity constants, reaching the femto-molar range $\left(10^{-15} \mathrm{M}\right)$ (red circles).

Since this procedure is based on a statistical process, the accuracy of the results depends on the sampling rate. We simulated a protein binding $\left(\boldsymbol{K}_{\boldsymbol{x}}=10^{7} \mathrm{M}^{-1}\right)$ while changing the number of available particles from 10000 to 1000 and 100. The results depicted in Figure 2-14b demonstrate that by increasing the number of available binding sites, lower concentrations can be detected.

\section{Conclusions}

In this chapter, we reviewed the concept of localized surface plasmon resonance in metallic nanoparticles. We described analytically, using the quasi-static approach, the optical properties of single nanoparticles, and by using numerical methods like DDA, we were able to quantitatively model the optical properties of the Au nanoparticles. In addition, we have introduced the concept of using individual gold nanoparticles for sensing applications and determined their optimal characteristics for sensing. We have established the limits of detection in the case of an un-amplified assay and we concluded that an amplification step is needed in order to boost the detection limits.

For the amplified assay, we have observed that the plasmon peak shift is dependent on a number of parameters like size, shape and number of the added particles as well as on the inter-particle distance, the polarization of light and the position of the added particle on the immobilized one. Although the range of obtained peak shifts was 
quite large, the minimum expected peak shift at one binding event would be sufficient to be detected by any commercial fiber based spectrometer.

In this chapter, we have introduced also the concept of parallel detection of multiple individually addressed nanoparticles to determine concentrations orders of magnitude below the inverse $\boldsymbol{K}_{\boldsymbol{x}}$. In addition, using a Monte Carlo procedure we were able to establish theoretically the limits of detection of such a sensing approach, both for unamplified (nano-molar) and amplified assay (pico-molar).

The validity of the theoretical findings will be experimentally investigated in the next chapters. 


\section{References}

[1] T. Okamoto, I. Yamaguchi, and T. Kobayashi, "Local plasmon sensor with gold colloid monolayers deposited upon glass substrates," Opt. Lett., vol. 25, Mar. 2000, pp. 372-374.

[2] C.L. Haynes and R.P. Van Duyne, "Nanosphere Lithography: A Versatile Nanofabrication Tool for Studies of Size-Dependent Nanoparticle Optics," The Journal of Physical Chemistry B, vol. 105, May. 2001, pp. 5599-5611.

[3] J.J. Mock, D.R. Smith, and S. Schultz, "Local Refractive Index Dependence of Plasmon Resonance Spectra from Individual Nanoparticles," Nano Letters, vol. 3, Mar. 2003, pp. 485-491.

[4] A.D. McFarland and R.P. Van Duyne, "Single Silver Nanoparticles as Real-Time Optical Sensors with Zeptomole Sensitivity," Nano Letters, vol. 3, Jul. 2003, pp. 1057-1062.

[5] J. Yguerabide and E.E. Yguerabide, "Light-Scattering Submicroscopic Particles as Highly Fluorescent Analogs and Their Use as Tracer Labels in Clinical and Biological Applications: I. Theory," Analytical Biochemistry, vol. 262, Sep. 1998, pp. 137-156.

[6] J. Yguerabide and E.E. Yguerabide, "Light-Scattering Submicroscopic Particles as Highly Fluorescent Analogs and Their Use as Tracer Labels in Clinical and Biological Applications: II. Experimental Characterization," Analytical Biochemistry, vol. 262, Sep. 1998, pp. 157-176.

[7] N. Nath and A. Chilkoti, "A Colorimetric Gold Nanoparticle Sensor To Interrogate Biomolecular Interactions in Real Time on a Surface," Analytical Chemistry, vol. 74, Dec. 2001, pp. 504-509.

[8] G. Raschke, S. Kowarik, T. Franzl, C. Sönnichsen, T.A. Klar, J. Feldmann, A. Nichtl, and K. Kürzinger, "Biomolecular Recognition Based on Single Gold Nanoparticle Light Scattering," Nano Letters, vol. 3, May. 2003, pp. 935-938.

[9] M.D. Malinsky, K.L. Kelly, G.C. Schatz, and R.P. Van Duyne, "Chain Length Dependence and Sensing Capabilities of the Localized Surface Plasmon Resonance of Silver Nanoparticles Chemically Modified with Alkanethiol Self-Assembled Monolayers," Journal of the American Chemical Society, vol. 123, Jan. 2001, pp. 1471-1482. 
[10] A.J. Haes, W.P. Hall, L. Chang, W.L. Klein, and R.P. Van Duyne, "A Localized Surface Plasmon Resonance Biosensor: First Steps toward an Assay for Alzheimer's Disease," Nano Letters, vol. 4, May. 2004, pp. 1029-1034.

[11] M. Faraday, “The Bakerian Lecture: Experimental Relations of Gold (and Other Metals) to Light," Philosophical Transactions of the Royal Society of London, vol. 147, 1857, pp. 145-181.

[12] G. Mie, "Beiträge zur Optik trüber Medien, speziell kolloidaler Metallösungen," Ann Phys, vol. 330, 1908, pp. 377-445.

[13] H.C. van de Hulst, Light Scattering by small particles, New York: 1981.

[14] L. Kelly, E. Coronado, L. Zhao, and G. Schatz, "The Optical Properties of Metal Nanoparticles: The Influence of Size, Shape, and Dielectric Environment," The Journal of Physical Chemistry B, vol. 107, Jan. 2003, pp. 668-677.

[15] I.O. Sosa, C. Noguez, and R.G. Barrera, "Optical Properties of Metal Nanoparticles with Arbitrary Shapes," The Journal of Physical Chemistry B, vol. 107, Jun. 2003, pp. 6269-6275.

[16] P.K. Jain, K.S. Lee, I.H. El-Sayed, and M.A. El-Sayed, "Calculated Absorption and Scattering Properties of Gold Nanoparticles of Different Size, Shape, and Composition: Applications in Biological Imaging and Biomedicine," The Journal of Physical Chemistry B, vol. 110, Mar. 2006, pp. 7238-7248.

[17] E. Hao, S. Li, R.C. Bailey, S. Zou, G.C. Schatz, and J.T. Hupp, "Optical Properties of Metal Nanoshells," The Journal of Physical Chemistry B, vol. 108, Jan. 2004, pp. 1224-1229.

[18] C. Noguez, "Surface Plasmons on Metal Nanoparticles: The Influence of Shape and Physical Environment," The Journal of Physical Chemistry C, vol. 111, Feb. 2007, pp. 3806-3819.

[19] A.A. Lazarides, K. Lance Kelly, T.R. Jensen, and G.C. Schatz, "Optical properties of metal nanoparticles and nanoparticle aggregates important in biosensors," Journal of Molecular Structure: THEOCHEM, vol. 529, Sep. 2000, pp. 59-63.

[20] M.A. Yurkin, D. de Kanter, and A.G. Hoekstra, "Accuracy of the discrete dipole approximation for simulation of optical properties of gold nanoparticles," Journal of Nanophotonics, vol. 4, 2010.

[21] C. Ungureanu, R.G. Rayavarapu, S. Manohar, and T.G. van Leeuwven, "Discrete dipole approximation simulations of gold nanorod optical properties: Choice of input parameters and comparison with experiment," Journal of Applied Physics, vol. 105, 2009, pp. 102032 - 102032-7. 
[22] L.A. Trachuk, A.G. Melnikov, and N.G. Khlebtsov, "Dependence of the optical properties of metal nanoparticles on the external dielectric medium: effects of the particle size, shape, and structure," SPIE Proceedings, vol. 5829, 2005, pp. 127-137.

[23] L.K. Zhao, K. Lance, and G.C. Schatz, "The Extinction Spectra of Silver Nanoparticle Arrays: Influence of Array Structure on Plasmon Resonance Wavelength and Width†," Journal of Physical Chemistry B, vol. 107, 2003, pp. 7343-7350.

[24] T. Sannomiya, C. Hafner, and J. Voros, "In situ Sensing of Single Binding Events by Localized Surface Plasmon Resonance,” Nano Letters, vol. 8, Sep. 2008, pp. 3450-3455.

[25] P.J. Draine, B.T., \& Flatau, "Discrete dipole approximation for scattering calculations," J. Opt. Soc. Am. A, vol. 11, 1994, pp. 1491-1499.

[26] P.B.J.Johnson and R.W. Christy, "Optical Constants of the Noble Metals," Physics Review Letters, vol. 6, 1972, pp. 4370-4379.

[27] J. Voros, "The Density and Refractive Index of Adsorbing Protein Layers," Biophysical journal, vol. 87, Jul. 2004, pp. 553-561.

[28] R.B.M. Schasfoort and A. Tudos, eds., Handbook of Surface Plasmon Resonance, RSC Publishing, 2008.

[29] I. Langmuir, "The constitution and fundamental properties of solids and liquids.II Liquids," Journal of the American Chemical Society, vol. 39, Sep. 1917, pp. 1848-1906.

[30] M.-E. Aubin-Tam and K. Hamad-Schiffer, "Structure and function of nanoparticleprotein conjugates," Biomedical Materials, vol. 3, 2008, p. 17.

[31] C.-Y. Yang, E. Brooks, Y. Li, P. Denny, C.-M. Ho, F. Qi, W. Shi, L. Wolinsky, B. Wu, D.T.W. Wong, and C.D. Montemagno, "Detection of picomolar levels of interleukin-8 in human saliva by SPR," Lab on a Chip, vol. 5, 2005, pp. 1017-1023.

[32] W. Rechberger, A. Hohenau, A. Leitner, J.R. Krenn, B. Lamprecht, and F.R. Aussenegg, “Optical properties of two interacting gold nanoparticles," Optics Communications, vol. 220, 2003, pp. 137-141.

[33] A.M. Funston, C. Novo, T.J. Davis, and P. Mulvaney, "Plasmon Coupling of Gold Nanorods at Short Distances and in Different Geometries," Nano Letters, vol. 9, Mar. 2009, pp. 1651-1658. 
[34] P.K. Jain, S. Eustis, and M.A. El-Sayed, "Plasmon Coupling in Nanorod Assemblies: Optical Absorption, Discrete Dipole Approximation Simulation, and Exciton-Coupling Model," The Journal of Physical Chemistry B, vol. 110, Aug. 2006, pp. 18243-18253.

[35] V. Bogatyrev, L. Dykman, B. Khlebtsov, and N. Khlebtsov, "Measurement of mean size and evaluation of polydispersity of gold nanoparticles from spectra of optical absorption and scattering," Optics and Spectroscopy, vol. 96, pp. 128-135. 


\section{Chapter 3}

\section{Sensing with colors}

$\boldsymbol{A b s t r a c t}^{3}$ : As discussed in the previous chapter, metal nanoparticles possess the property of changing their optical properties as a function of both internal characteristics (size, shape, dielectric function) and refractive index of the local environment. A special class of applications in the field of biosensing uses the dependence of the nanoparticle's plasmonic peak localization on the local refractive index change. The response of this type of sensors is usually monitored by the change of the extinction spectrum of an ensemble of nanoparticles where analytes interact with functionalized nanoparticles in solution or immobilized at an interface; detection is done with a spectrophotometer. This type of sensors has a limited sensitivity. This limitation can be overcome by using single nanoparticle based biosensors. This type of sensors measures the changes of the scatter spectrum of a collection of individually addressable functionalized nanoparticles in the presence of analytes.

\footnotetext{
3Part of this chapter was published as "The use of a colour camera for quantitative detection of protein binding nanoparticles", Ungureanu, F., Halamek, J., Verdoold, R., Kooyman, R.P.H., Proc. SPIE 7192, 71920 O (2009); doi:10.1117/12.806522
} 
Here we report on a new detection method of binding events of analytes to functionalized gold nanoparticles using a standard colour camera attached to a darkfield microscopy setup. This setup is capable of parallel detection of the spectral shifts of thousands of antibody-functionalized gold spheres as a result of binding events of protein analyte molecules. This setup can be the basis for multiplexing and quantification.

We demonstrate the feasibility of this approach by monitoring spectral changes of individual nanoparticles upon protein adsorption, and upon immuno-binding. We also discuss the performance of a colour camera relative to that of two monochrome cameras. 


\section{Introduction}

Often, the design of biosensing devices involves the use of optical reporters, such as colorimetric, fluorometric or chemiluminescent, either as direct or indirect labels [1-3]. A common measuring approach is to obtain the cumulative signal of an ensemble of labels present in the region of interest. In such a system the detection of (nearly-) single molecule binding events is essentially impossible. This circumstance is one of the reasons why the ultimate sensitivity of such a 'macro-device' will be limited. An alternative to this approach is the detection of individual binding events using fluorescent labels. Yet, the low quantum yield, blinking, and the irreversible photo-destruction of the fluorescent molecules make single binding events difficult to detect, even if expensive equipment is used.

A new class of materials, noble metal nanoparticles, potentially overcomes these limitations. When such a particle is placed in an electromagnetic field (in the visible range) with a certain well-defined wavelength, a localized surface plasmon (a collective oscillation of the metal's free electrons) can be excited. In these conditions the scattering crosssection for a single $60 \mathrm{~nm}$ Au particle irradiated at a wavelength of $540 \mathrm{~nm}$ is around 2*10${ }^{20} \mathrm{~m}^{2}$ [4], while the scattering cross-section of a fluorescein molecule is $10^{5}$ smaller. This allows the visualization of single nanoparticles in e.g. a darkfield microscope, using relatively simple detection equipment.

The wavelength at which a surface plasmon can be excited in the nanoparticle is highly dependent on its intrinsic properties (composition, size, shape) as well as on the refractive index of the immediate environment [5-8]. It is this last property that makes nanoparticles interesting for biosensing. Okamoto et al [9] were the first to show the feasibility of a gold nanoparticles covered glass substrate as a stand-alone sensing platform for the monitoring of molecular binding in real time.

Another important advantage of single nanoparticle sensor systems is the possibility of wavelength multiplexing. Unique sensing platforms can be fabricated by 
controlling the size, shape and chemical modification; each type can be distinguished from the other ones, based on their unique wavelength dependent scatter maxima $[10,11]$.

The most common method of characterizing binding events on individual particles is resonant Rayleigh scattering spectroscopy [12-14]. For example, the method used by Sannomiya et al [15] for detecting individual binding events on single nanoparticles involves the use of a regular spectrometer attached to a microscope and scanning electron microscopy (SEM) for visual verification. Baciu et al [16], for the detection of streptavidin binding to membrane-coated particles, used, instead of a spectrometer, an electronically addressable liquid crystal device, which gives them the advantage of simultaneously collecting the signal from several nanoparticles.

However, in view of the large number of nanoparticles present on the surface, which can undergo binding events, this detection method cannot be considered for quantification purposes.

In this chapter, we introduce an alternate detection method by employing a colour camera in a quantitative way. Its main advantages are (1) parallel detection of hundreds to thousands of nanoparticles; (2) the possibility of wavelength multiplexing.

\section{Experimental section}

\subsection{Detection principle}

The small dimensions of nanoparticles require specialized microscopic methods for their visualization. Current methods allow the study of the morphology of nanoparticles (Scanning Electron Microscopy (SEM), Atomic Force Microscopy (AFM), Transmission Electron Microscopy (TEM)) and of their optical properties (UV-VIS spectroscopy, dark-field microscopy (DF), total internal reflection microscopy (TIR)). Here we restrict our study to the monitoring of changes in the optical properties of individual gold nanoparticles. For this, we used DF and TIR microscopy. 


\subsubsection{Dark field microscopy}

Created in 1903 by August Kohler, darkfield (DF) microscopy is probably the most inexpensive and most efficient way to visualize small features of specimens. This technique uses an illumination procedure that enhances the contrast by collecting only the light scattered by objects situated in the sample plane. In these conditions, the background appears black in contrast to the bright objects. The technique is schematically depicted in Figure 3-1a.

Here, the central part of the light entering the microscope is blocked by a stop disk, which leaves only an outer ring of rays to enter the system. The light collected by the condenser lens is focused towards the sample plane. At this point, a part of the incoming light is scattered by the objects present and the rest of the light is directly transmitted. The angular acceptance of the objective lens is chosen such that it only collects the scattered light. Thus, in the resulting image objects that scatter appear bright on a black background, as observed in Figure 3-1b. Darkfield microscopy, due to its nature, can be considered an artefact-free method, as it allows only the visualization of scattering objects.

(a)

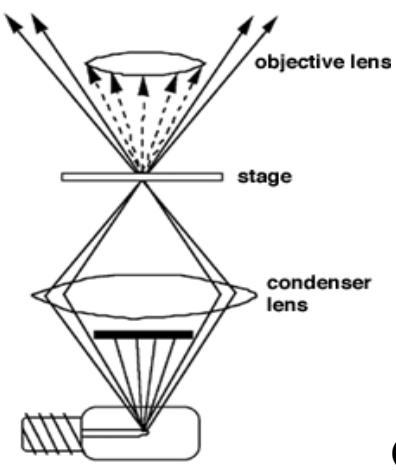

(b)

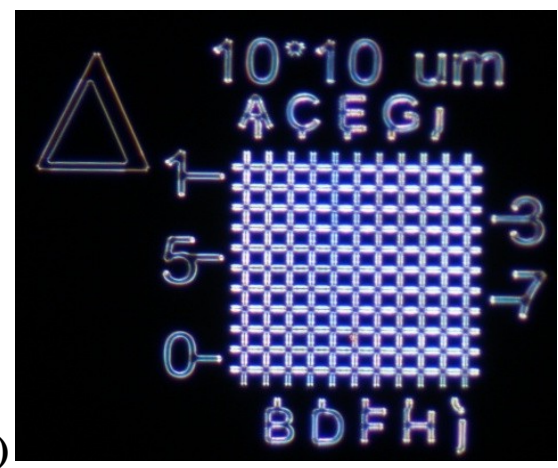

Figure 3-1 a) Schematic representation of the DF principle [17] ; b) polymer grid under DF illumination.

The main limitation of this technique is the low light level used; this can be overcome by strong illumination of the sample. 
An alternative to this method, without light loss, would be to use a setup based on the principle of total internal reflection.

\subsubsection{Total Internal Reflection}

Total Internal Reflection (TIR) is an optical phenomenon that occurs when the light is incident on the boundary of two media with different refractive indices (the refractive index of the second medium is smaller than the one of the first medium) at an angle larger than the critical angle, with respect to the normal to the surface. The critical angle is defined as the angle of incidence at which the refracted light travels along the boundary.

In normal conditions, when the light hits the interface between two media, part of the beam is refracted at the boundary and part is reflected back in the first medium, as described by Fresnel's equations. When the light travels from a medium with a higher to one with a lower refractive index, a critical angle can be defined, above which the light will undergo total internal reflection. This situation is represented schematically in Figure 3-2 a.

(a)

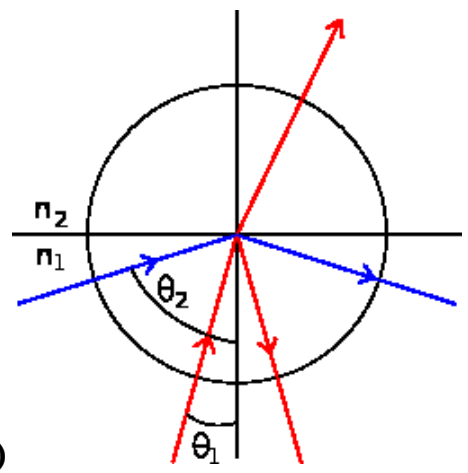

(b)

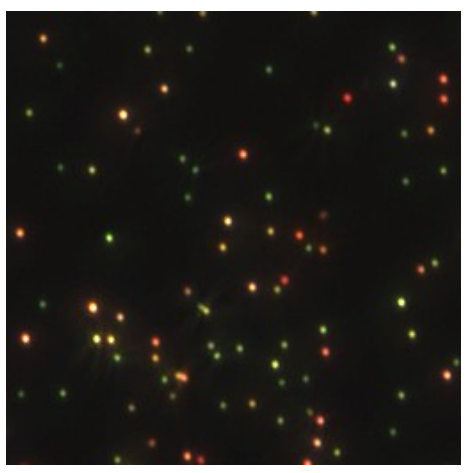

Figure 3-2 a) The schematic representation of the TIR principle. The light travelling from the initial medium, with refractive index $\mathrm{n}_{1}\left(\mathrm{n}_{1}>\mathrm{n}_{2}\right)$, at a small angle in respect to the normal to the surface (red lines), will partially be refracted in medium $\mathrm{n}_{2}$ and partially reflected. When the incident angle is larger than the critical angle the light is totally reflected in the medium $\mathrm{n}_{1} . \mathrm{b}$ ) Typical image of Au NPs imaged using a TIR setup. The red hue is a chromatic aberration resulted from small misalignments and mismatches in the experimental setup. 
The critical angle can be obtained from rearranging Snell's law [18]:

$$
\theta_{c}=\arcsin \frac{n_{2}}{n_{1}}
$$

An important side effect of the occurrence of total internal reflection is the generation of an evanescent wave that propagates along the interface between the two media. When a third medium, with a higher refractive index $n_{3}$, is added on top of the medium $\mathrm{n}_{2}$, with a dimension within the penetration depth of the evanescent wave, a coupling of the evanescent with the new medium will occur allowing energy transfer from the second to the third medium. This is what we call frustrated total internal reflection (FTIR).

The benefits of using this method for the visualization of gold nanoparticles refer to the amount of light available for illumination of gold nanoparticles (here, all the available light is employed compared with darkfield illumination where only a small percentage of the available light is utilized) as well as for the possibility of controlling the polarization of light. Figure 3-2b shows an example of an area with multiple individual 80 $\mathrm{nm}$ Au nanoparticles imaged using a TIR setup.

\subsection{Optical setup}

To collect the scattered light from individual gold nanoparticles (images and spectra), we used an inverted Olympus GX71 microscope in a darkfield (DF) reflection configuration in combination with a fibre spectrophotometer (Ocean Optics QE65000, $1024 \times 64$ pixels). 


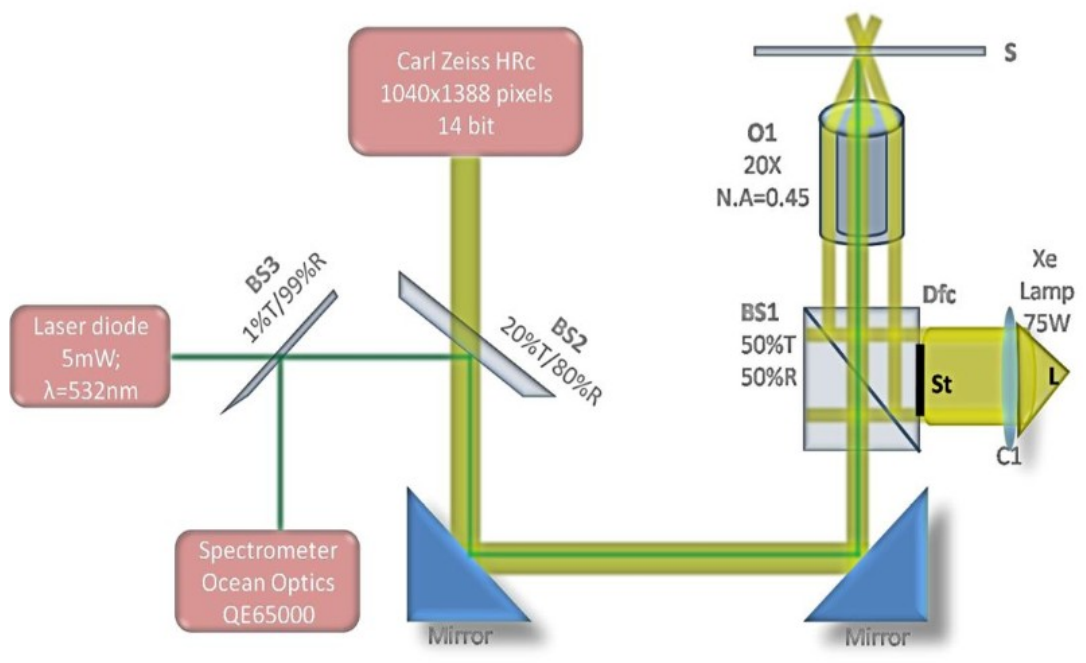

Figure 3-3 The experimental setup

The sample (S) was illuminated through beamsplitter BS1 with a Xe lamp (L) (75 W) as presented in Figure 3-3.The light is collimated by a lens (C1) and reaches the DF filter cube (DFc). This acts as a darkfield condenser. Here, the central part of the light is blocked by the central stop $(\mathrm{St})$ of the DFc aperture. The rest of the light is then transmitted, through a beamsplitter (BS1) at a $45^{\circ}$ angle into the BF/DF objective (O1) focussing on the sample. The scattered light deflected by the sample is collected back by the same objective (MPLFLN20xBD/N.A=0.45) and send to the colour camera (Carl Zeiss HRc, 1040x1388 pixels, 14 bits colour depth). A 40x magnification is obtained using a combination of the 20x objective described above with a $2 x$ build-in lens. This lens was not corrected for chromatic aberration therefore the effect is visible in all images. Chromatic aberration can pose serious problems in determining the center of mass (CM) of moving diffraction limited beads. However, in this manuscript, we imaged stationary nanoparticles and the accuracy in determining the CM is not so strict. When a color camera is employed to image nanoparticles, the CM of each nanoparticle can be determined accurately by analysing each color channel individually and on each channel, the $\mathrm{CM}$ is calculated. The $\mathrm{CM}$ of the nanoparticle is then the average of the CM from each color channel. 
For the wavelength calibration of the colour camera we used the image of a tightly focused spot produced by a diode laser (HeNe) $(5 \mathrm{~mW}, \lambda=532 \mathrm{~nm})$ to select individual nanoparticles for spectral analysis. Here, the scattered light deflected by the sample is divided in two: $80 \%$ of the light is sent through a $50 \mu \mathrm{m}$ fibre to the spectrometer (Ocean optics; QE65000) and 20\% is forwarded to the colour camera.

The time required to obtain a single nanoparticle spectrum with sufficient SNR was $50 \mathrm{~s}$, whereas the exposure time to obtain an image with the camera was 750 $\mathrm{ms}($ Spectrometer Off) or $4 \mathrm{~s}($ Spectrometer On).

\subsection{Materials}

As a substrate for the sensor chip, we used BK7 cover-slips (26mm x 76mm, 0.17 mm thick) purchased from Menzel Glaser, Germany [19] . The gold nanoparticle colloidal solutions (60 and $80 \mathrm{~nm}$ diameter) were purchased from BBInternational (Cardiff, UK) [20] and used as purchased without any further purification. In our experiments we used rabbit anti-goat polyclonal antibody (Sigma Aldrich, G4018), donkey anti-goat polyclonal antibody(Sigma Aldrich, G6638), goat IgG antibody (Sigma Aldrich, B3640) or horseradish peroxidase (HRP) (VI, Sigma Aldrich, P6782), provided by Sigma Aldrich. All chemicals and proteins were used as purchased, without further purification. Water used for the preparation of buffers, solutions and for washing was exclusively of milliQ quality.

Chemical preparation protocols are described in the appendix of this chapter.

\subsection{HIM experiments}

The morphology (size and shape characterization only) of gold nanoparticles was determined using Helium Ion Microscopy (HIM) [21]. This technology has several advantages over classical Scanning Electron Microscopy (SEM). Because of the very bright source and high momentum of the helium ions, the quality of the obtained images is significantly better than those obtained with conventional methods, which use photons or electrons as emitting source. Compared with conventional SEM, the images obtained with HIM technology show more detail and have a higher contrast because here the beam 
of helium ions interacts with the sample and the excitation volume is low. The damage inflicted on the sample is relatively low compared with other ion beams because of the low mass of the helium ions. This method offers high quality images with a high amount of valuable information from topographic features to electrical properties of the sample.

\subsection{Data analysis}

A Matlab code was developed to analyse the images obtained with our current setup. The parameter $\mathrm{r} / \mathrm{g}$, defined as the ratio between the pixel value of the red channel and that of the green channel, was used as a measure of the colour change of individual nanoparticles. We experimentally demonstrated the invariance of the $\mathrm{r} / \mathrm{g}$ ratio of a nanoparticle to luminosity changes; additionally, it shows the highest sensitivity to spectral shifts as compared with other parameters (e.g. Blue/ Green, Blue/Red) (see section Results and Discussion).

This Matlab code allowed us to track the $\mathrm{r} / \mathrm{g}$ ratio in time for all nanoparticles of interest present in our field of view (FOV) from a series of images and to quantify how many of them show a colour change. The procedure for determining the $\mathrm{r} / \mathrm{g}$ consisted of several steps. The images were processed to a minimum in order to keep as much information as possible.

The Image toolbox represents the colors from the image into RGB values. For analysis purposes, the color image was decomposed into individual color channels: red, green and blue.

First, all raw images must be background corrected.

For this correction, several steps were done. For this function, we used an adapted version of the statistical model developed by David Millan [22]. Pixels with values that vary independently with normal distribution were considered background pixels. To emulate the normal experimental conditions, an empty flowcell (no nanoparticles in the FOV), with a running solution of PBS, was considered. After an image was recorded, the average pixel values $\mathrm{A}(\mathrm{x}, \mathrm{y})$ and its standard deviation $\sigma(\mathrm{x}, \mathrm{y})$ were calculated. In order to be considered signal, pixels must meet the requirement: $|A(x, y)-B(x, y)| \geq z \cdot \sigma(x, y)$ 
where $\mathrm{B}(\mathrm{x}, \mathrm{y})$ is the pixel value and $\mathrm{z}$ is a constant, set by us at 3. However, sometimes structures, other than nanoparticles (e.g. dust particles) are present in the FOV and for their removal, a tophat filter from the Matlab image toolbox was used. This filter is an "opening" procedure followed by a subtraction of the result from the initial image. This filter uses a single structuring element (SE) returned by the function strel which eliminates all features smaller than the dimension of the SE. We used a discoidal SE with a radius of 15 pixels. This takes into account the diameter of the nanoparticle's spot and a neighbouring area. The diameter of the nanoparticle is represented over 9-10 pixels and a 5 pixel bordering was considered.

Then, an image preceding any change in the system is selected and is defined as reference (usually is the first image taken before adding any modification in the system).

Secondly, a digital mask was applied to this image, to locate and extract the particles of interest. The following parameters were considered for this: the roundness, the area and the $\mathrm{r} / \mathrm{g}$ ratio.

The roundness defines the circularity of the object under consideration and is described by the eccentricity (ecc $=$ the ratio between the minor and the major axis length). The values are between 0 and 1; 0 for a line profile and 1 for circular shape). This parameter is used to exclude adjacent non-touching particles that cannot be optically resolved. A circular profile of the scattering is attributed to single nanoparticles while the elongated profiles correspond to two or more close, non-touching particles. A certain deviation from circularity is accepted due to inherent imperfections (e.g. Chromatic aberrations) in the optical setup. Typical values of circularity range between 0.8 and 1 . In order to exclude particles with sizes outside the range of interest, as well as other types of particles (impurities e.g. dust), the area of the scattering profile of the nanoparticles in the FOV is measured. The area is given by the actual number of pixels delimiting the nanoparticle. This increases with the size of the particle. Typical values, for $60 \mathrm{~nm}$ Au particles, go between 20 and 30 pixels.

The two parameters described above are supplementary to the $\mathrm{r} / \mathrm{g}$ ratio parameter, which is the most important parameter of all. 
This parameter is based upon the chromaticity coordinates (normalized colours), defined as:

$$
r=\frac{R}{R+G+B} ; g=\frac{G}{R+G+B} ; b=1-r-g
$$

where $\mathrm{r}$ - redness, g- greenness and b- blueness [23]. These parameters are known to be independent to illumination conditions, as demonstrated by Ailisto and Piironen (1987) [24]. We demonstrated (further in this chapter) that also the $\mathrm{r} / \mathrm{g}$ ratio is independent on the illumination conditions. When the spectral distribution changes, thus a spectral change occurs the $\mathrm{r}, \mathrm{g}$ and $\mathrm{b}$ parameters react accordingly [25]. The $\mathrm{r} / \mathrm{g}$ ratio has been also used in application such as the detection of bronchial dysplasia and carcinoma in situ [26]. Nevertheless, to the best of my knowledge, until know there is no publication in which the $\mathrm{r} / \mathrm{g}$ ratio has been used to quantify spectral shifts in nanoparticles.

This parameter is related to the spectral information of the particle (the typical values related to the size of the nanoparticle are given in Figure 3-18 of this chapter). For example, to select a population of $60 \mathrm{~nm}$ particles $(\mathrm{r} / \mathrm{g}=0.55)$, the selection thresholds are set between 0.5 and 0.6.

In a uniform sample (particles with the same size and shape and uniformly distributed over the FOV), more than $95 \%$ of the particles are selected. For the selected particles, we have calculated their $\mathrm{r} / \mathrm{g}$ from consecutive images and the results were stored in an output file.

To quantify the number of particles that show a colour change we use a subtraction algorithm. Here, two images are needed: the reference image and the image at the end of the experiment. The $\mathrm{r} / \mathrm{g}$ of the selected nanoparticles from the final image is subtracted from the initial values. Then a threshold $(\mathrm{t})$ is defined $(\Delta \mathrm{r} / \mathrm{g}>\mathrm{t})$, which corresponds to a certain wavelength shift (determined from calibration data and correlated with theoretical data). The value of the threshold is dependent on the type of immunoassay performed. In a non-amplified assay, a small spectral shift upon binding is expected, the threshold is lower than in the case of amplified assay, where larger spectral shift are measured. The threshold is set to account the minimum expected (theoretically determined) change upon binding. For the non-amplified assay, the $\mathrm{t}$ was set to 0.02 
$\overline{\text { (corresponding to } 1 \mathrm{~nm} \text { shift) and for the amplified assay t was } 0.1 \text { (corresponding to } 5}$ $\mathrm{nm}$ shift). We count only those particles that show a change larger than that limit. The disadvantage of the subtraction algorithm is that no information is given regarding the nanoparticle's state during the experiment. Therefore, in the extended software full information of the $\mathrm{r} / \mathrm{g}$ evolution in time is given (example of a signal is presented in figure 3-21). All data presented in this thesis were analysed using the full version of the software. Figure 3-4b shows screenshots from the analysis program.

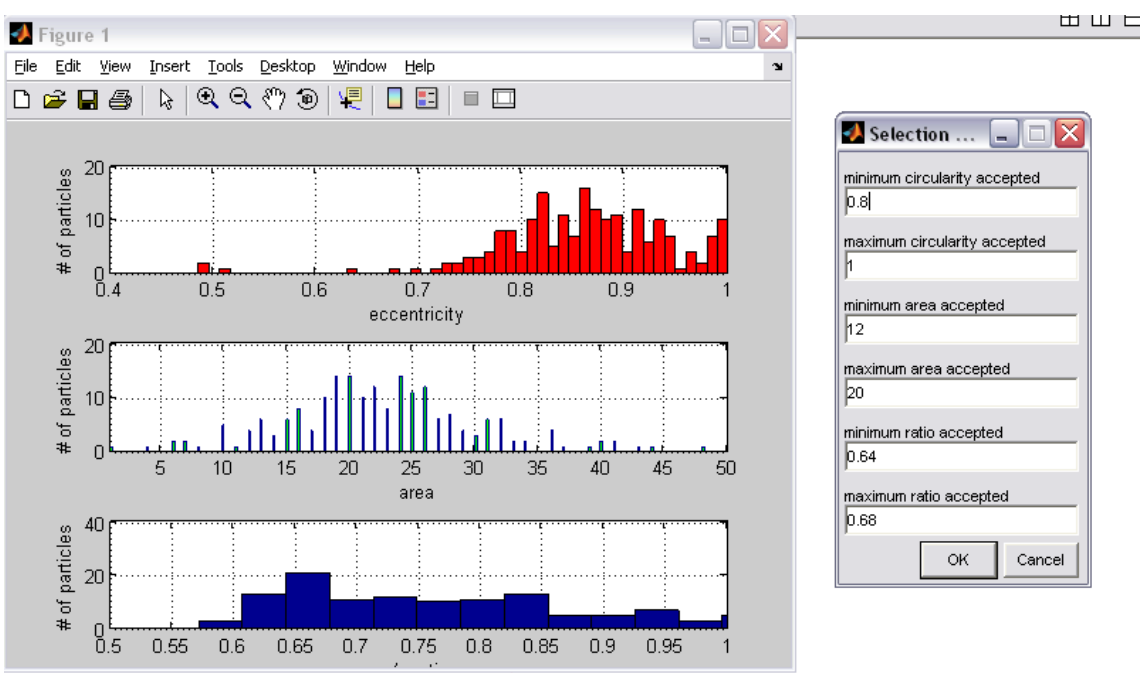

(a)

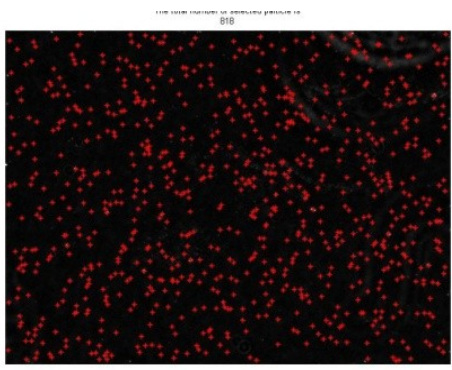

(b)

Figure 3-4 Screenshots of the analysis program. (a) the digital mask applied on the DF image showing the selection criteria. Here a very narrow range of particle sizes was selected (only $60 \mathrm{~nm}$ Au nanoparticles). (b)The nanoparticles selected for analysis after the digital mask was applied; (c) The selected nanoparticles, showing binding, on the DF image. The role of the false colours, which show the localization of the selected nanoparticles, is to guide the eye of the observer. 
The data obtained from the image analysis and the spectra of single nanoparticles were analysed using Origin software. Using the procedures explained above, we performed the following series of experiments:

- Selection of the $\mathrm{r} / \mathrm{g}$ parameter based on the following requirements: high sensitivity to spectral shifts, stability in time, invariance to luminosity conditions;

- Calibration of the colour camera for the detection of wavelengths shifts due to molecular interaction;

- Proof of principle of using the colour camera as a detector for a kinetic process (protein adsorption assay);

- Conducting an immunoassay and the estimation of the sensitivity, in terms of concentrations;

- Comparison, in terms of Signal to Noise Ratio (SNR) and sensitivity to spectral shifts, between two different detection approaches that allow parallel detection of multiple individual nanoparticles.

\section{Results and discussion}

Nanoparticles used in this manuscript, are diffraction limited objects $(60-100 \mathrm{~nm})$, and can be considered as point objects. The image of these objects consists of diffraction pattern, which can be observed as a central spot with one or more diffraction rings around it. In literature, they are known as Airy rings [27]. The dimension of the spot is known as the point spread function (PSF) and is related to the wavelength of the illumination $(\lambda)$ and the numerical aperture $(\mathrm{NA})$ of the objective $(\mathrm{NA}=0.45)$ according to the following equation: $\lambda / \mathrm{NA}$. In our setup using the settings described in the section for optical setup, the PSF for the nanoparticles (60 respectively $80 \mathrm{~nm}$ Au particles) are PSFAu60nm $=1.22 \mu \mathrm{m}$

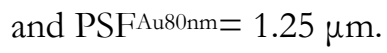


In most of our experiments, the images of immobilized Au nanoparticles were analyzed. Figure 3-5a shows a typical DF image of $60 \mathrm{~nm}$ Au particles immobilized on a glass surface. In the field of view $\left(F O V=230 \times 170 \mu \mathrm{m}^{2}\right)$ the average distance between two individual Au nanoparticles is estimated at $10 \mu \mathrm{m}$.

Figure 3-5b shows a SEM image of $60 \mathrm{~nm}$ particles immobilized on a glass surface with a longer incubation time.

(a)

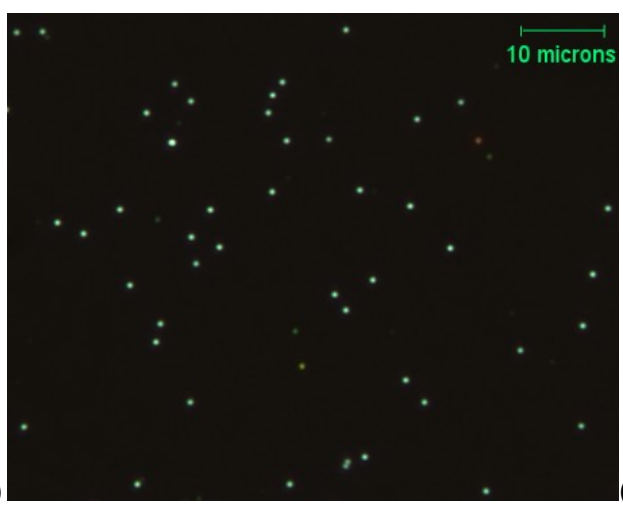

(b)

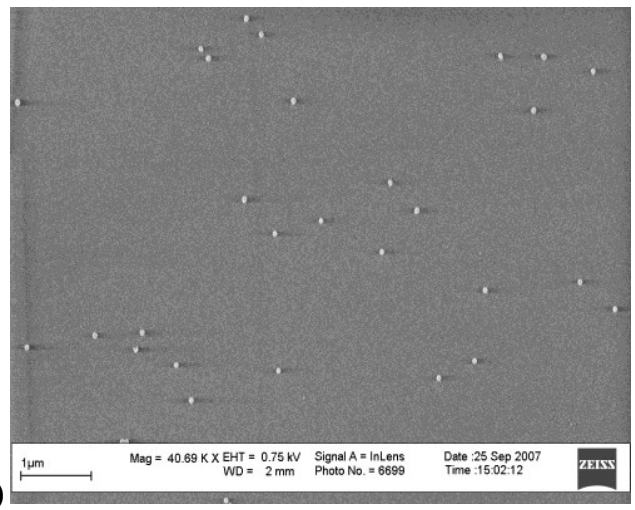

Figure 3-5 (a) A typical DF image of $60 \mathrm{~nm}$ Au particles immobilized on a glass surface (1.4 $* 10^{10}$ particles $/ \mathrm{ml}$ and incubation time 5 minutes) (b) SEM image of $60 \mathrm{~nm}$ Au particles immobilized on glass with a larger incubation time (20 minutes).

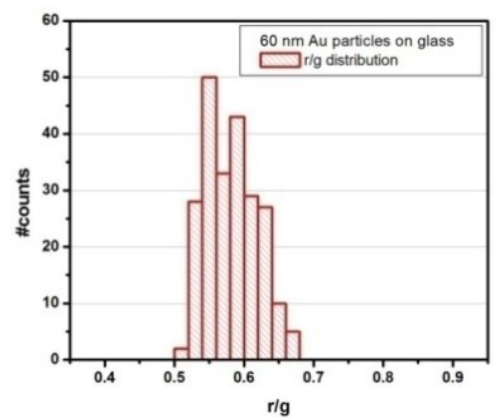

(a) (b)

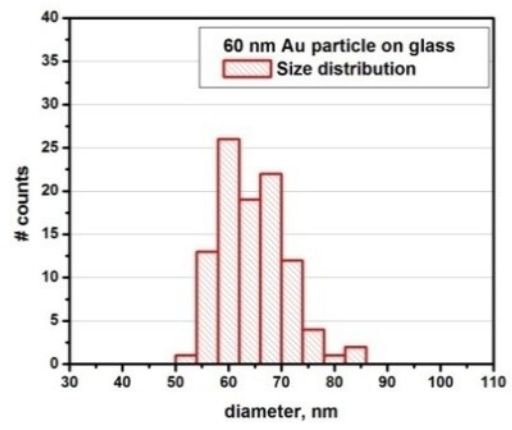

Figure 3-6 (a) The colour distribution of $60 \mathrm{~nm}$ particles from a DF image with an FOV= 230x170 $\mu \mathrm{m}^{2}$; (b) The size distribution of Au nanoparticles determined from SEM images for a total of100 nanoparticles 
Figure 3-6a illustrates the colour distribution ( $\mathrm{r} / \mathrm{g}$ distribution) of the nanoparticles inside the sample.

The narrow spreading of colour (corresponding to $\Delta \lambda_{\text {FWHM }} \sim 5 \mathrm{~nm}$, see Section 3.2.1.) suggests that nanoparticles inside the FOV have similar sizes and shapes. Figure 3$6 \mathrm{~b}$ depicts the distribution in sizes of an ensemble of SEM pictures totalizing 100 individual nanoparticles. It can be observed that the particles can be found as single entities and not in clusters. This information, correlated with a low polydispersity in colours observed from figure 3-5a, suggests that the colour response from DF images corresponds to individual nanoparticles.

\subsection{Colour camera as detector for spectral shifts}

Currently, the detection of spectral changes of individual nanoparticles, due to binding of biomolecules, implies the use of a device capable of spectral measuring. This can be either a regular spectrophotometer, adapted for single particle detection [28], or involves the use of special filters [16]. Although very accurate in measuring the spectral information, these devices are limited in acquiring the spectra of a large number of nanoparticles.

This disadvantage can be overcome by using a colour camera, with true color capabilities, as it can detect the spectral information (retained in their colour) of all individual nanoparticles present in the FOV simultaneously. Typically, for true color measurements, a 3 CCD chip imaging system is employed to measure individually the tree spectral ranges (red, green and blue). Although precise, these systems are very expensive. A viable alternative is to use the Axiocam HRc color camera, which provides true color information via the colour co-site sampling acquisition technique [29]. In this approach, the Bayer filter is physically moved with one pixel in 4 directions (up-down, right-left) to obtain for each pixel R, G, B data. By doing so, each image point contains red, green and blue measured data. Unlike common single CCD imaging systems, where interpolation methods are used to recreate the colour information, for the AxioCam HRc camera this 
method is no longer necessary. Here, the 4 images are added to produce a true color image, where the color information is measured and not guessed.

As we are interested to measure spectral changes of individual nanoparticles, due to molecular interactions, a feature that shows high sensitivity to spectral changes is needed. In addition, this parameter has to fulfil the following requirements:

- Stability to different luminosity conditions;

- Stability in time.

We performed a series of experiments to evaluate some features that can be extracted from images. We monitored the behaviour of ratios between different channels (Red/Green, Blue/Green and Blue/Red) for these conditions. For these experiments, transparent solutions with different $\mathrm{pH}$ were made. Next, an amount of $\mathrm{pH}$ indicator (SIGMA 36828) was added; this led to a change in the colour of the solution according to its $\mathrm{pH}$ (Figure 3-7a). The solutions used in our experiments were selected based on the following criteria:

- their spectra should resemble as much as possible those of the Au nanoparticles used in our experiments (Figure 3-7b);

- the extinction level has to be the same in each solution;

(a)

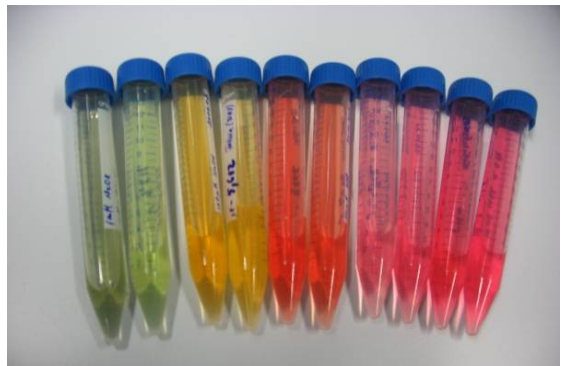

(b)

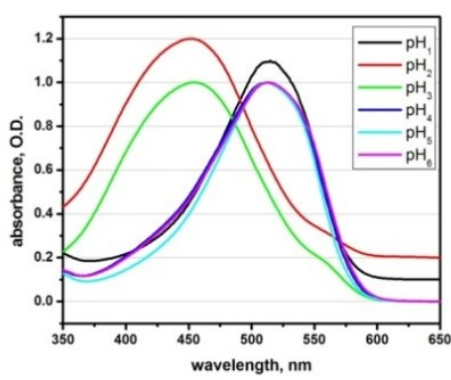

wavelength, $\mathrm{nm}$

Figure 3-7 (a) Coloured solutions with $\mathrm{pH}$ indicators suggesting they have different $\mathrm{pH}$ 's; (b) The spectra of a number of $\mathrm{pH}$ solutions.

The next step was to investigate the sensitivity to colour change of the features extracted from the images of the $\mathrm{pH}$ solutions. From the available $\mathrm{pH}$ solutions, we 
selected two showing a wavelength shift of $3.5 \mathrm{~nm}$ in the extinction spectrum. Then the ratios $\mathrm{r} / \mathrm{g}$ (red over green), b/g (blue over green) and b/r (blue over red) were examined for the entire FOV. The results presented in Table 3-1 show that the $\mathrm{r} / \mathrm{g}$ ratio has the best sensitivity to spectral changes compared to the other ratios. Therefore, we decided to consider only the $\mathrm{r} / \mathrm{g}$ ratio as parameter.

\begin{tabular}{|c|c|c|c|}
\hline$\Delta \lambda, \mathrm{nm}$ & $\boldsymbol{\Delta} \mathrm{r} / \mathrm{g}$ & $\boldsymbol{\Delta} \mathrm{b} / \mathrm{g}$ & $\Delta \mathrm{b} / \mathbf{r}$ \\
\hline 3.5 & 0.07 & 0.02 & 0.05 \\
\hline
\end{tabular}

Table 3-1. The sensitivity to spectral changes of $\mathrm{r} / \mathrm{g}, \mathrm{b} / \mathrm{g}$ and $\mathrm{b} / \mathrm{r}$ parameters.

The stability to illumination conditions was checked. We decreased the amount of light in the system by placing various neutral density filters and then imaged different $\mathrm{pH}$ solutions. Figure 3-8a shows a typical image of one solution in one illumination state. Figure $3-8 \mathrm{~b}$ illustrates the $\mathrm{r} / \mathrm{g}$ dependence on the illumination conditions for three different $\mathrm{pH}$ solutions. Here, a value $\mathrm{r} / \mathrm{g}=0.40 \pm 0.001$ for $\mathrm{pH}_{1}, \mathrm{r} / \mathrm{g}=0.294 \pm 0.004$ for $\mathrm{pH}_{2}$ and $\mathrm{r} / \mathrm{g}=0.32 \pm 0.005$ for $\mathrm{pH}_{3}$ were obtained. The standard deviation is two orders of magnitude lower than the mean value. These results show that the $\mathrm{r} / \mathrm{g}$ parameter is invariant to illumination conditions. Particularly, the standard deviation is very low for the $\mathrm{pH}_{1}$ solution whose spectrum corresponds relatively closely to that of an Au nanoparticle.

(a)

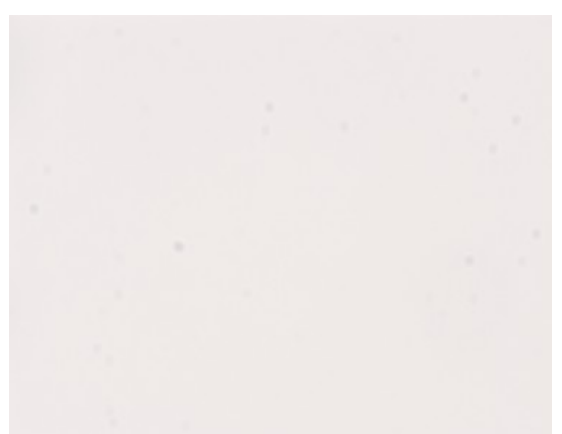

(b)

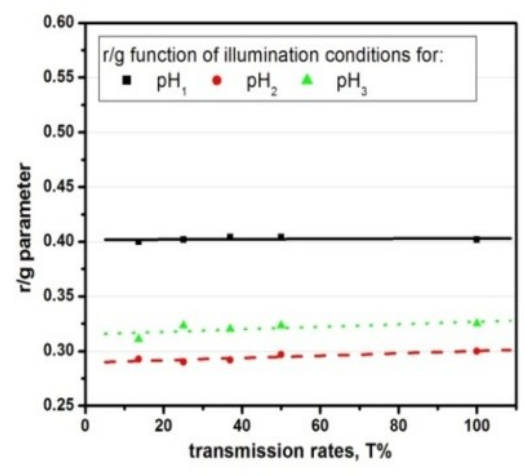

Figure 3-8 (a) The image of a pH solution in bright-field (BF) conditions; (b) The $\mathrm{r} / \mathrm{g}$ dependence on the illumination conditions for three $\mathrm{pH}$ solutions. 
Subsequently, we examined the invariance of the $\mathrm{r} / \mathrm{g}$ value to the extinction level of the $\mathrm{pH}$ solution, while the rest of the parameters were kept constant (e.g. illumination conditions). A series of solutions were prepared with a certain $\mathrm{pH}$ and different extinction levels as illustrated in Figure 3-9a. As in the previous case the response of the $\mathrm{r} / \mathrm{g}$ ratio does not depend on the extinction level of the coloured solution as shown in Figure 3-9b.

(a)

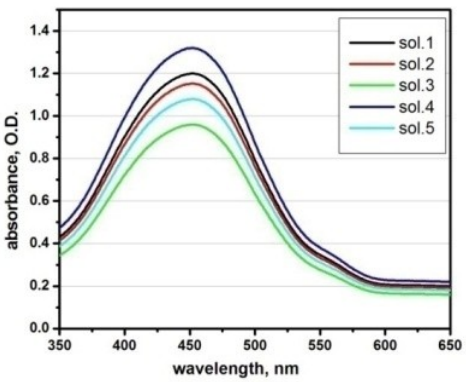

Figure 3-9 (a) The absorption spectra for a series of solutions of a certain $\mathrm{pH}$; (b) The $\mathrm{r} / \mathrm{g}$ dependence on the absorption level of the $\mathrm{pH}$ solution.

Another good indicator of the validity of the chosen parameter is the stability in time. We flowed $(\mathrm{v}=14 \mu \mathrm{l} / \mathrm{min})$ a water solution over a slide with immobilized $80 \mathrm{~nm}$ au particles, placed under DF conditions, and for half an hour we monitored the $\mathrm{r} / \mathrm{g}$ value of the nanoparticles from the FOV. The image was actively kept in focus by the motorized stage using a custom-made Labview program. The results illustrated in Figure 3-10 show a low variance of the $\mathrm{r} / \mathrm{g}$ value within the standard deviation $(0.01)$, as expected.

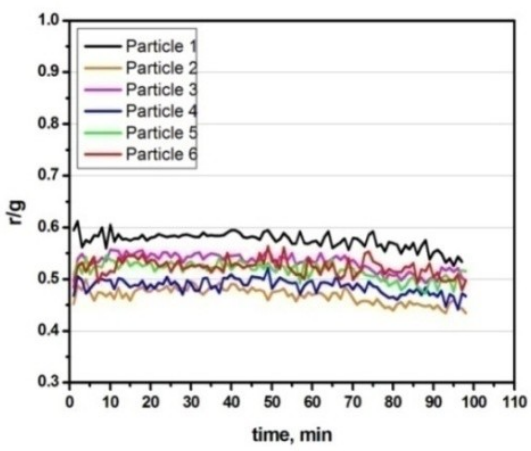

Figure 3-10 The stability of the $\mathrm{r} / \mathrm{g}$ value of several gold nanoparticles. 
Lastly, we performed a series of experiments to assess the minimum spectral shifts that the colour camera can measure. From all available $\mathrm{pH}$ solutions, we selected those showing a $1 \mathrm{~nm}$ shift in the extinction spectrum (Figure 3-11). Then the solutions were imaged and the $\mathrm{r} / \mathrm{g}$ ratio was calculated.

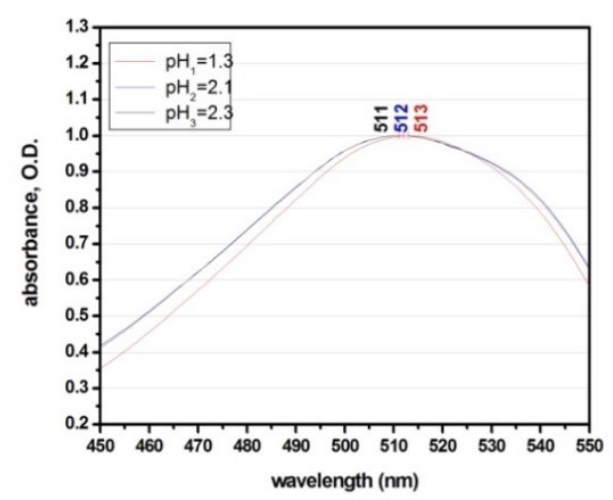

Figure 3-11 The absorption spectra of three solutions with $1 \mathrm{~nm}$ shift of the maximum

\begin{tabular}{|l|l|l|l|l|}
\hline Sample & $\lambda_{\max }(\mathrm{nm})$ & $\mathbf{R}$ (D.N.) & G (D.N.) & $\mathbf{r} / \mathbf{g}$ \\
\hline 1. & 511 & $14937 \pm 122$ & $13787 \pm 118$ & $1.08 \pm 0.01$ \\
\hline 2. & 512 & $15013 \pm 123$ & $13430 \pm 116$ & $1.12 \pm 0.01$ \\
\hline 3. & 513 & $15001 \pm 120$ & $13139 \pm 110$ & $1.14 \pm 0.01$ \\
\hline
\end{tabular}

Table 3-2.pH solutions with $1 \mathrm{~nm}$ shift. (D.N. denotes digital numbers)

The results of this experiment, as shown in table 3-2, demonstrate that a colour camera can detect at least $1 \mathrm{~nm}$ spectral shift. We did not determine if this is the limiting spectral sensitivity of our colour camera, as we encountered some difficulties in preparing two solutions that have a mutual spectral shift of less than $1 \mathrm{~nm}$.

The results of these experiments show clearly the feasibility of the $\mathrm{r} / \mathrm{g}$ ratio as a feature to monitor spectral shifts. The next step is to calibrate the camera to detect wavelength shifts due to molecular interactions. 


\subsection{Calibration of the camera}

\subsubsection{Wavelength versus refractive index calibration}

Two sets of samples with 60 or $80 \mathrm{~nm}$ Au particles immobilized on glass were placed into custom-made flow-cells and used for calibration. The initial embedding medium is water. The experiments were done using the optical setup described in section 2.1 .

Firstly, we determined the spectra, using the fibre spectrometer described in section 2.1, of 10 individual particles from each set and the results were compared to theory (DDSCAT simulations, cf. Chapter 2). Figure 3-12 shows typical spectra acquired with the present setup. From the comparison with simulated spectra of the same particles, we observe a difference of a few nanometers in the position of the plasmonic peak (PP). One reason for the difference could be that the nanoparticles used in the experiments have a difference in morphology from those used in the simulation.

(a)

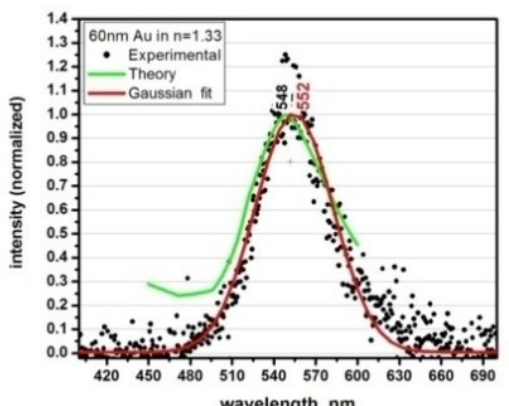

wavelength, $\mathrm{nm}$

(b)

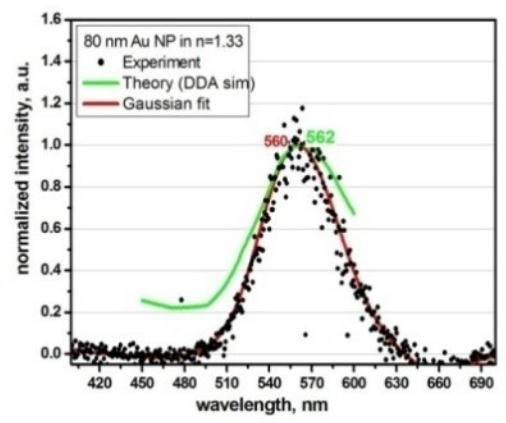

Figure 3-12 The simulated and experimental spectra of an individual (a) $60 \mathrm{~nm}$ and (b) $80 \mathrm{~nm} \mathrm{Au}$ particle in water

For the wavelength versus refractive index calibration of the colour camera, we carried out the following experiment. The sensor surface was subjected to changes in the refractive index by adding glucose solutions of various concentrations (Table 3-3), of which the refractive indices were determined using an Abbe refractometer. Each refractive index step was followed by washing with MQ water. The experiment was performed in a flow regime ( $v=14 \mu \mathrm{l} / \mathrm{min})$. 


\begin{tabular}{|c|c|c|c|c|c|}
\hline Glucose, $\%$ & $\mathbf{1 0}$ & $\mathbf{2 0}$ & $\mathbf{3 0}$ & $\mathbf{4 0}$ & $\mathbf{5 0}$ \\
\hline $\mathbf{n}$ & 1.346 & 1.362 & 1.377 & 1.392 & 1.415 \\
\hline $\begin{array}{c}\text { Time track, } \\
\text { Min }\end{array}$ & $11-20$ & $31-40$ & $51-60$ & $71-80$ & $91-95$ \\
\hline
\end{tabular}

Table 3-3 The timetable of the refractive index calibration experiment.

Figure 3-13a illustrates a typical signal of an individual gold nanoparticle, measuring the colour change, during the refractive index experiment. The yellow areas in the graph indicate the incubation steps of glucose solutions. Step-like increases can be observed each time a higher refractive index solution is added. During the washing steps, the $\mathrm{r} / \mathrm{g}$ value returns to the original levels. At higher refractive index changes, the $\mathrm{r} / \mathrm{g}$ base level is increased suggesting that not all the glucose around the nanoparticles has been washed away.

(a)

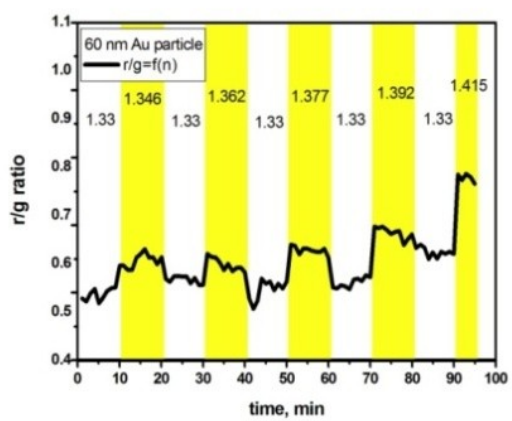

Figure 3-13 (a) A characteristic signal during refractive index experiment of a $60 \mathrm{~nm} \mathrm{Au}$ nanoparticle; (b) the corresponding spectral information of the same nanoparticle.

For the wavelength calibration, we monitored the scattering spectra of 10 individual nanoparticles during the refractive index change experiment. An example of the spectral information (raw spectra) of a $60 \mathrm{~nm}$ Au particle during the refractive index experiment is depicted in Figure 3-13b, showing a $10 \mathrm{~nm}$ shift at a 0.085 refractive index change. 
Two types of information were obtained from these experiments: (1) calibration curves for the colour camera for the two sets of samples and (2) the sensitivity curves for the $60 \mathrm{~nm}$ Au particles. Figure 3-14 shows both the experimental and simulated sensitivity curves.

By comparing the theoretical data, obtained in a uniform environment, and the experimental data for the nanoparticle immobilized on glass, we can conclude that the presence of the substrate has a limited effect on the sensitivity behaviour of the nanoparticles. The somewhat higher sensitivity slope in the bulk solution could be the averaged result of the size distribution of the nanoparticles present in the solution. This value suggests that a larger amount of bigger particles is present in solution, shifting the sensitivity to refractive index to higher values.

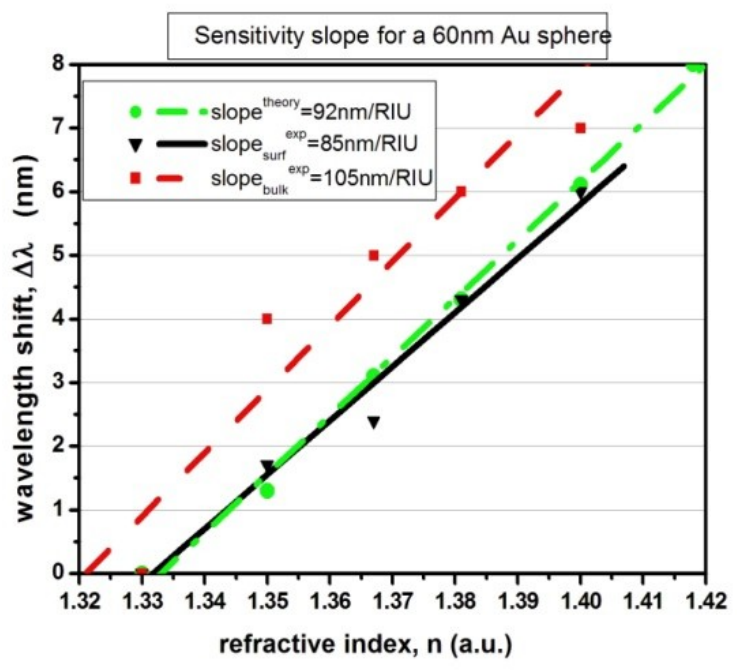

Figure 3-14 The sensitivity curves for $60 \mathrm{~nm}$ Au particle determined theoretically (circles), for a single nanoparticle immobilized on surface (triangles) and for a distribution of particles in bulk solution (squares).

The accuracy in determining the refractive index of the embedding media was $\Delta \mathrm{n}=5^{*} 10^{-3}$. The average of 10 separate spectral or $\mathrm{r} / \mathrm{g}$ measurements was used for further data processing. 


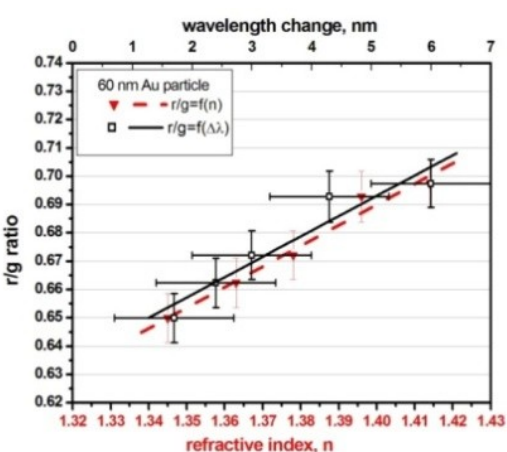

(a)

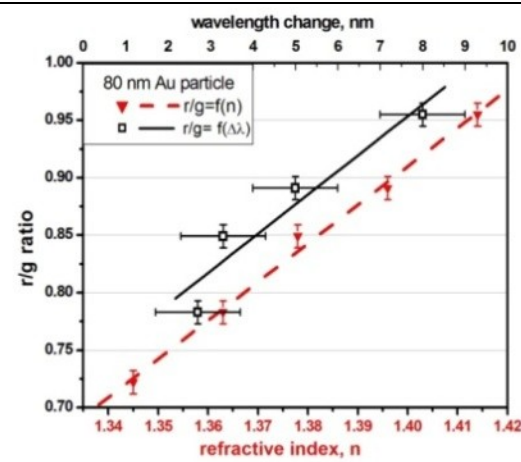

(b)

Figure 3-15 The calibration curves of the colour camera for (a) $60 \mathrm{~nm}$ Au particle and (b) $80 \mathrm{~nm}$ Au particle

The calibration results for the colour camera are shown in Figure 3-15 for the two sets of particles. For the refractive index calibration, we determined that the minimum refractive index change that can be detected with 60 and $80 \mathrm{~nm}$ Au particles is $10^{-2}$ and $10^{-2}$, respectively. With respect to the wavelength calibration curves for the two sets of nanoparticles, we conclude that the limit of resolution is $\sim 1 \mathrm{~nm}$. According to the calibration data, we can conclude that:

- $\quad 0.02 \mathrm{r} / \mathrm{g}$ change corresponds to $1 \mathrm{~nm}$ wavelength shift;

- $0.33 \mathrm{r} / \mathrm{g}$ change corresponds to 0.1 refractive index change;

- The noise levels in determining the $\mathrm{r} / \mathrm{g}$ values are $0.005-0.01$;

\subsubsection{Size calibration}

For the size calibration, $80 \mathrm{~nm}$ bare particles were immobilized on a glass slide with a layer of indium tin oxide (ITO), a conductive material needed for HIM/SEM imaging. First, an area was selected and 60 nanoparticles were imaged with HIM, Figure 316a. The same areas were found back in DF image (Figure 3-16b) and the scattering spectra of individual nanoparticles, using the fibre spectrometer, described in section 2.1, were monitored. The particles were imaged in air. Figure 3-17 shows the HIM/DF/spectroscopy data for several nanoparticles. In this figure, we show a comparison between the morphological and spectroscopic data for the same particle. 

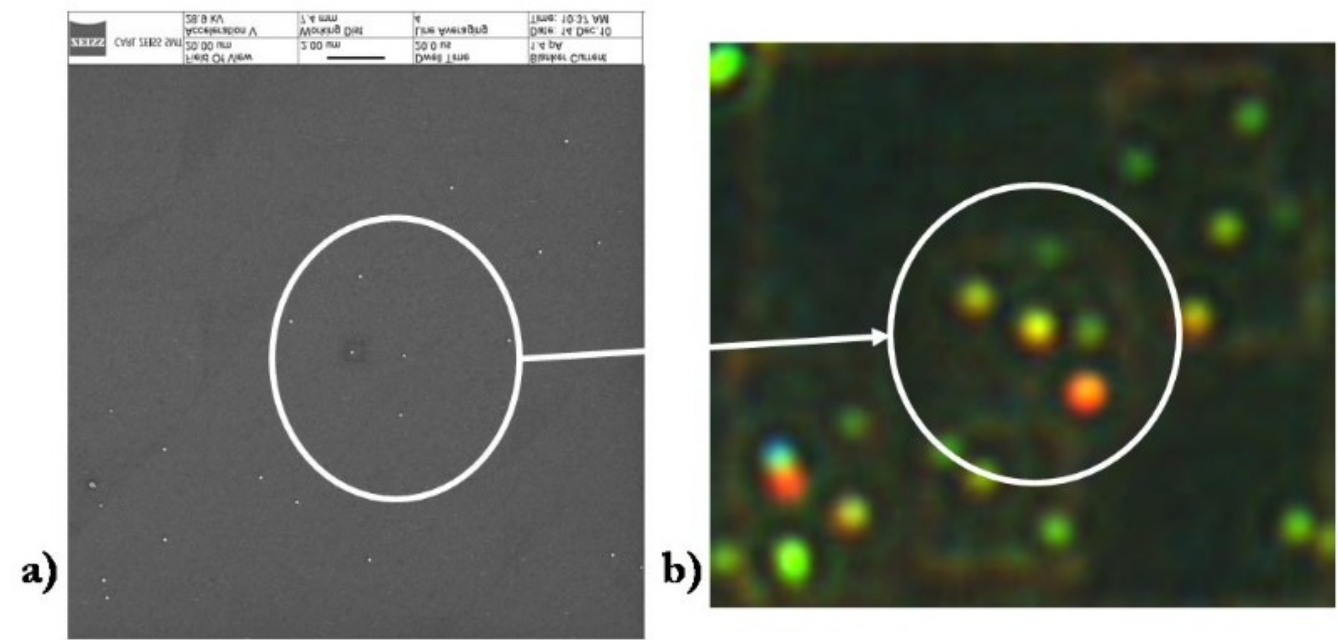

Figure 3-16 (a) HIM and (b) the corresponding DF image of the same nanoparticles $\left(\sim 20 \mu \mathrm{m}^{2}\right)$

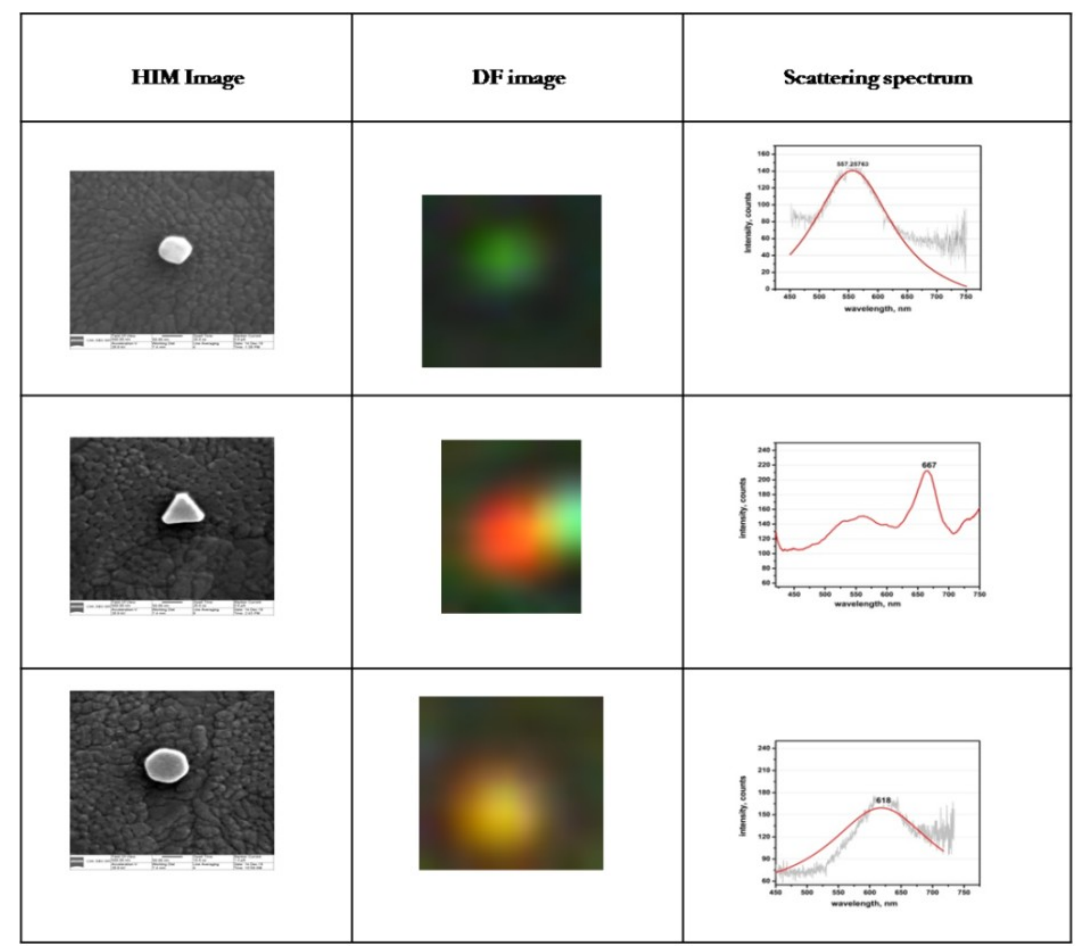

Figure 3-17 The HIM/ spectroscopic and DF information of several nanoparticles 
Two sets of information can be obtained from Figure 3-18a: (1) an experimental correlation between the localization of the PP and the size of the nanoparticle and (2) the comparison with theoretical data and a size calibration curve of the colour camera.

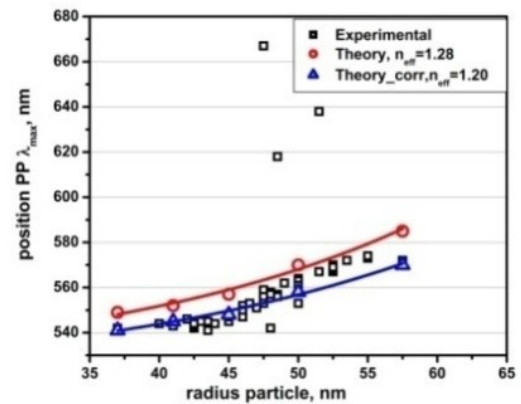

(a)

Figure 3-18 (a) Correlation between the position of the PP and radius of the particles obtained from the spectroscopic and HIM data. (b) Size calibration of the colour camera

Some differences can be observed when comparing the experimental results ( $\mathrm{Au}$ nanoparticles on ITO slide) with theoretical results (Au nanoparticles in homogenous medium, $\mathrm{n}=1.28)$ ). This value, $\mathrm{n}=1.28$, was obtained by averaging the refractive index of the air $(n=1)$ and the averaged refractive index of the substrate on which the particles are placed. The substrate was composed from APTES $(n=1.46)$ [30], ITO glass $(n=1.775$, $\mathrm{k}=0.012$ )[31]. By adjusting the refractive index of the environment (using the formula 2.7 presented in Chapter 2, Section 3) a better matching between the experimental and theoretical results is observed. Here, a weighted averaging was performed. This correction is justified as the nanoparticles are embedded in a complex medium. The averaging was done considering that only one third of the volume of the nanoparticles is influenced by the close proximity to the substrate. The rest of the particle is immersed in the surrounding medium (air, in this particular case). Although not exact, this single approximation is enough to match the experimental with the theoretical results (triangles). The shape of the nanoparticles has an important role in determining the localization of the PP; the presence of the three outliers on our graph emphasizes this argument.

The size calibration of the colour camera is depicted in figure 3-18b. One curve represents the dependence of the PP position on the size of the nanoparticle (black 
squares) and the other is a representation of the dependence of the $\mathrm{r} / \mathrm{g}$ ratio on the size of the nanoparticle. The correlation between the two curves implies that we can estimate the size of the particles from the value of the $\mathrm{r} / \mathrm{g}$ parameter (provided that the experimental conditions are similar).

Based on these results, we can conclude that a colour camera is able to resolve two particles with $5 \mathrm{~nm}$ difference in radius.

The size discrimination opens the possibility of parallel detection of multiple molecular species using a colour camera. Here, nanoparticles with different sizes, each pre-coated with a specific protein molecule/ DNA fragment can be detected and analyzed in parallel.

A simple protein adsorption assay was designed to test that a colour camera can be utilized for the monitoring of molecular interaction processes. This hypothesis is tested in the following section.

\subsection{Protein adsorption assay}

Here we track the $\mathrm{r} / \mathrm{g}$ of multiple individual nanoparticles in time when protein molecules adsorb on their surface. The schematic representation of the experiment is pictured in Figure 3-19.
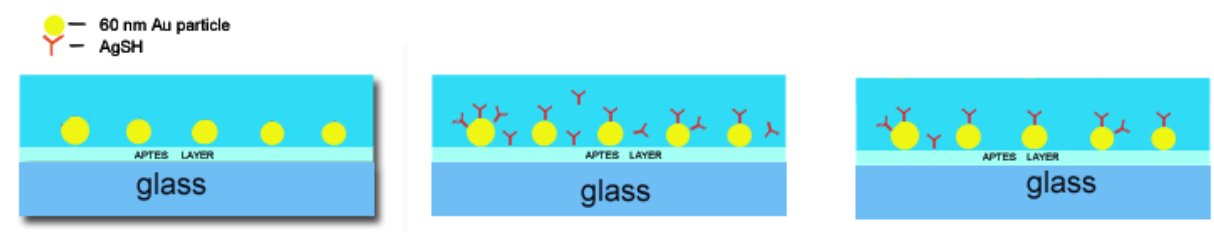

Figure 3-19 Schematic representation of the protein assay. The surface chip is incubated for 45 minutes with thiolated anti-goat antibody $(\mathrm{AgSH})$ and then washed with MQ water.

A flow-cell, schematically represented in Figure 3-20, containing a sensing surface with immobilized $60 \mathrm{~nm}$ Au particles was used for the protein assay. In such a closed system, no contamination from the experimental area can be introduced. The flow-cell is divided in three channels $(\mathrm{LxWxH}=68 \times 4 \times 0.1 \mathrm{~mm})$ of which two were used: one for 
the control, where no antibody is added and one for the adsorption experiment. The control experiment was carried out immediately after, as we were limited in monitoring only one channel at a time. The images were acquired at 1-minute intervals.

The flow assay was conducted at a flow rate of $4.2 \mu \mathrm{l} / \mathrm{min}$ as follows:

- 5 minutes of washing the surface with MQ water

- Incubation for 50 minutes with a solution containing thiolated $\alpha$-goat ( $\alpha$-goat$\mathrm{SH})$ for the sample and MQ water for the reference

- The channels were washed for 15 minutes with MQ water

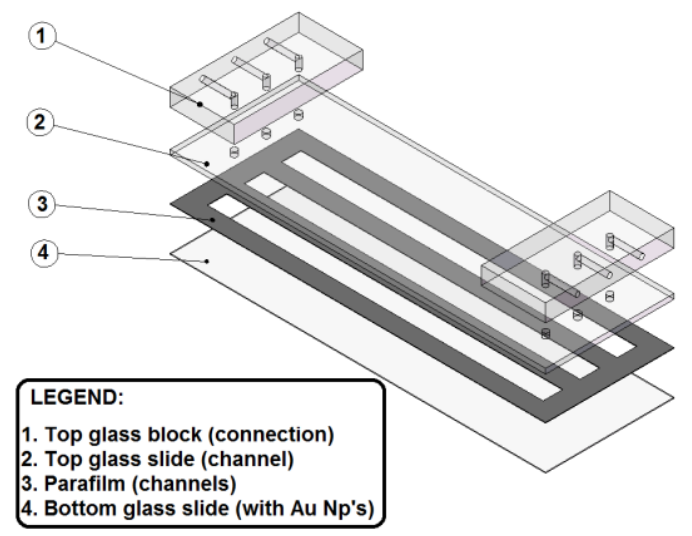

Figure 3-20 The schematic representation of the flow-cell.

Typical signals for individual nanoparticles are shown in Figures 3-21. They represent only a small part of the total amount of available data. Particles, from the sample chamber, with no significant signal change were also present. A possible explanation for this could be that the binding sites of those nanoparticles were blocked before the start of the experiment. 


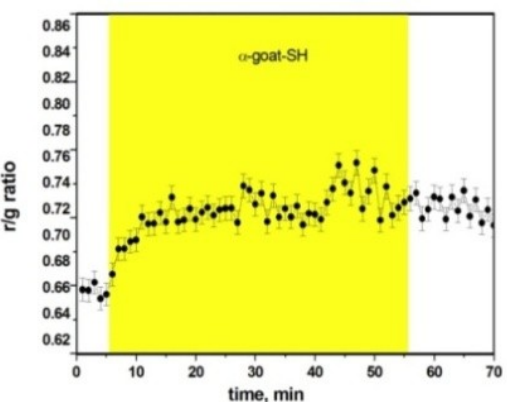

(a)

Figure 3-21 Two characteristic signals of two individual nanoparticles.

By combining the results from the control, Figure 3-22 and the assay experiments, Figure 3-21, we can conclude that the increase of the signal can only originate from the protein binding to the particle's binding sites.

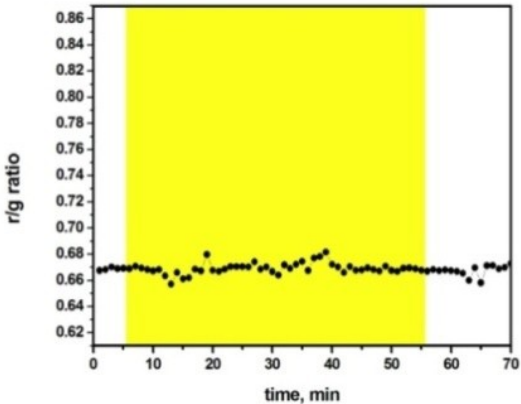

(a)

Figure 3-22 Typical signals from two individual nanoparticles in the control experiment

The signal from figure 3-21a has the profile of a characteristic binding curve of a protein. In the time intervals, 28-30 minutes and 43-52 minutes, two larger signal increases were observed, which disappear after a while. A possible explanation for this could be that impurities from the external environment entered in the protein solution and immobilized near the nanoparticle; at a random time, they re-entered the flow and were washed away. In this signal, no visible dissociation process is observed in the washing step.

Figure 3-21b shows a different type of behavior. The evolution of the signal until $\mathrm{t}=30 \mathrm{~min}$ shows a binding curve with a slower rate than the previously discussed signal. 
$\overline{\text { Starting at } \mathrm{t}=31 \mathrm{~min} \text {, the development of a second curve suggests that a second layer of }}$ protein was formed which, however, appears to be removed in the washing step starting at $\mathrm{t}=56 \mathrm{~min}$.

\subsection{Quantification}

For the quantification of the occupied binding sites of the particle, we combined the experimental data with the calibration and theoretical curves presented in Chapter 2, Figure 2-6. According to these data, the full coverage of the surface of a $60 \mathrm{~nm}$ Au particle with protein molecules ( $6 \mathrm{~nm}$ in diameter) would lead to a maximum of $6 \mathrm{~nm}$ wavelength shift. Here, from the wavelength calibration data of the $\mathrm{r} / \mathrm{g}$ ratio, we can observe a $4 \mathrm{~nm}$ shift at saturation level.

(a)

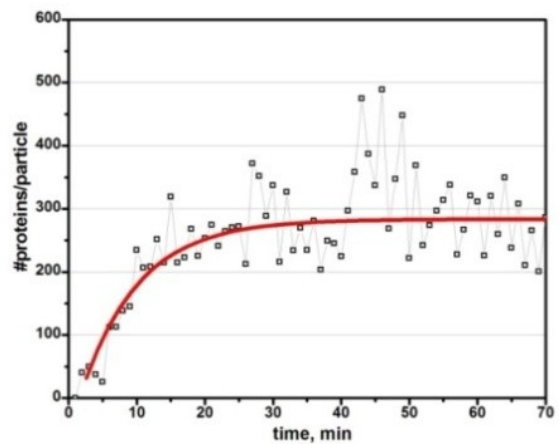

Figure 3-23 The quantified curves of two measured binding curves

From geometrical consideration, 400 proteins (spheres with $6 \mathrm{~nm}$ in diameter) can accommodate on one $60 \mathrm{~nm}$ Au particles. However, due to the immobilization of the nanoparticle on a substrate, part of the area is unavailable for binding which corresponds to about 100 binding sites (each site is considered to have the projected area of one protein molecule). If we subtract this number from the total available sites we conclude that only around 300 protein molecules can physically be accommodated on one immobilized $60 \mathrm{~nm}$ Au particle. This number compares well with the value obtained experimentally (Figure 3-23). While Figure 3-23a shows a clear saturation level at $\sim 300$ molecules, Figure 3-23b shows the formation of a second binding curve on top of the 300 
molecules level. This emphasizes the observation that for the formation of a complete layer of protein on the $60 \mathrm{~nm}$ Au particles not more than $\sim 300$ molecules are necessary.

In the next section, the specificity of an immuno-binding is investigated.

\subsection{Protein immunoassay}

Here, the specific binding of target molecules to the receptor molecules, present on the surface of individual nanoparticles was investigated. A set of experiments was designed to detect protein immunoassays using the colour camera as a detector.

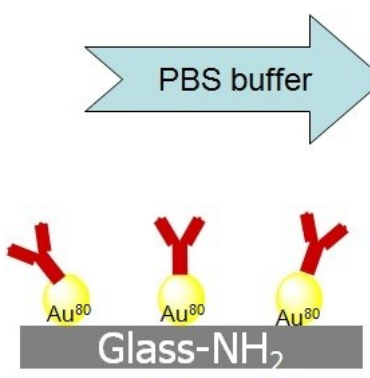

(a)

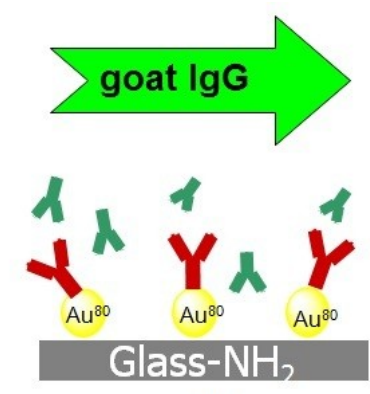

(b)

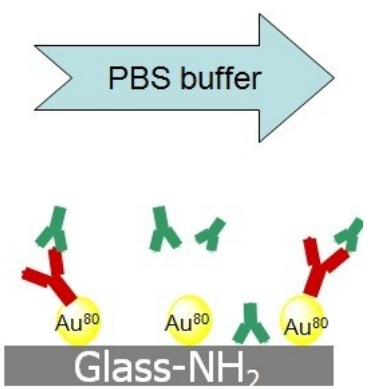

(c)

\section{Ygoat 1 goat IgG}

Figure 3-24 Illustration of the experiment design. The conjugated nanoparticles were immobilized on glass surface (a) and the target molecules were incubated for 120 minutes (b). Immediately afterwards, the sensor surface was washed with PBS buffer (c).

In this experiment on the sensing surface conjugated nanoparticles were immobilized using the protocol described in the Appendix of this chapter. We used two channels of the flowcell, described in section 3.3 of this chapter. Two sets of binding assays were conducted (Figure3-24):

- as the sample: the binding of goat $\operatorname{IgG}$ (goat $\operatorname{IgG}$ ) to rabbit anti-goat antibody functionalized $80 \mathrm{~nm} \mathrm{Au}$ particles $\left(\alpha g o a t-\mathrm{Au}^{80}\right)$ and

- $\quad$ as the control: the lack of binding of Horseradish Peroxidase (HRP) to $\alpha g o a t-\mathrm{Au}^{80}$ 
The images were acquired at 1-minute intervals (in total 180 images) for each channel. The following steps were taken in the flow experiments:

- 30 minutes washing with phosphate buffered saline (PBS) (flow rate $=14 \mu \mathrm{l} / \mathrm{min}$ );

- Incubation with a solution containing goat $\operatorname{IgG}\left(10^{-7} \mathrm{M}\right)$ for the sample and HRP(10${ }^{7} \mathrm{M}$ ) for the reference, respectively) for 120 minutes (flow rate $=2 \mu \mathrm{l} / \mathrm{min}$ );

- 30 minutes washing with PBS (flow rate $=14 \mu \mathrm{l} / \mathrm{min}$ ).

The results of these experiments, sample and control, are shown in Figure 3-25, respectively 3-26.

(a)

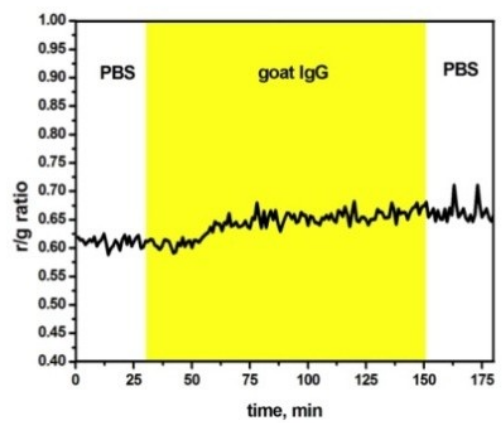

Figure 3-25 Two signals from two individual nanoparticles showing immunobinding (sample)

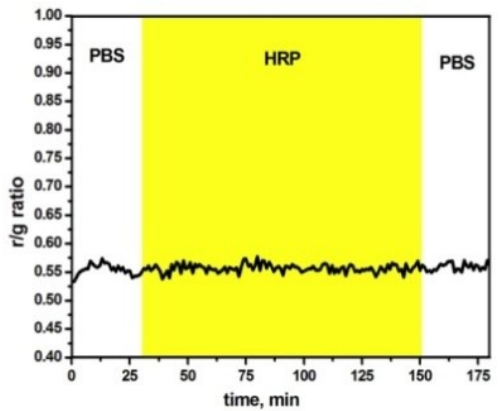

(a)

(b)

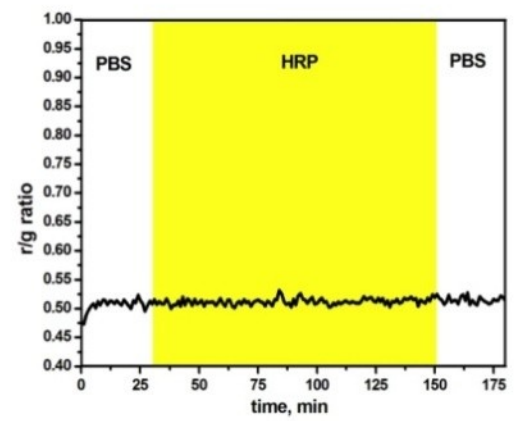

Figure 3-26 Two signals from individual nanoparticles with no binding event (control)

The signals presented in Figure 3-25 have the characteristic profile of a binding process. Several peaks are present in both signals, which can be the effect of some 
impurities passing the FOV. The maximum measured $\mathrm{r} / \mathrm{g}$ change was 0.07 , corresponding to a maximum $3.5 \mathrm{~nm}$ shift.

The signals depicted in Figure 3-26 show no change in the colour of the analyzed nanoparticles, being an evidence of no binding event occurring. The signal variation during the entire experiment was within the normal noise levels $( \pm 0.01)$.

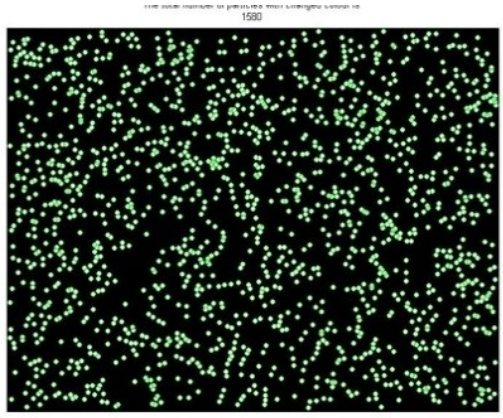

(a)

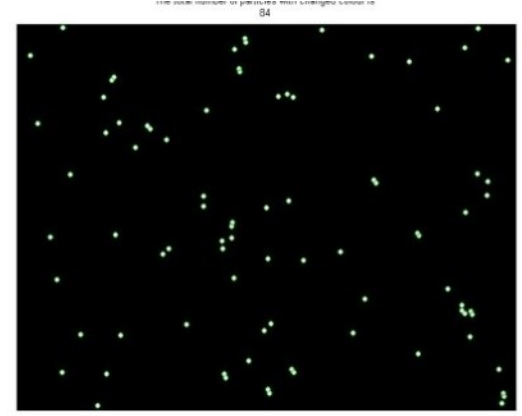

(b)

Figure 3-27 Selected nanoparticles with a colour change larger than the threshold in a (a) sample and (b) control. On average, we have 2000 analyzed particles in each FOV. We used a concentration of $800 \mathrm{nM}$ of goat IgG for the sample and $800 \mathrm{nM}$ of HRP

Figure 3-27 depicts the amounts of particles that show a colour change in the sample (a) and the reference (b). In both cases, 2000 particles were analyzed. While in the chamber where the specific target was added, the number of particles that show spectral shifts is very high (1580), in the reference only 84 out of 1980 particles show the same effect. The high difference in the amount for the selected particles can be considered as a good indication for the specificity of the binding reaction.

The results from the sample combined with the results obtained in control experiments prove that immune-bindings were detected using the colour camera as detection system.

As we are interested to detect low concentrations of target molecules, the limit of detection (LOD), in terms of concentration, of the detection system will be investigated in the next section. 


\subsection{Concentration dependence}

For the determination of the sensitivity of the detection system for this assay, the functionalized nanoparticles were incubated with different concentrations of target molecules and we counted the amount of particles that have changed colour at each target concentration.

Four flow-cells (as described in section 3.3) were used for a total of12 channels. Eight were incubated with a different concentration of target and three channels were incubated (various concentrations) with molecules which should not bind to the functionalized particles (Table 3-4). The sensing surface of each flowcell was incubated with functionalized gold nanoparticles $\left(\alpha\right.$-goat $\mathrm{Au}^{80}$ ) using the immobilization protocol described in the Appendix. For the reproducibility of the data, at each target concentration, three different sensing areas from each channel were measured.

Before the introduction of the target molecules in the channel, a DF image was acquired. After the incubation of the target molecules, the flowcells were washed and a second DF image was acquired. For data analysis, the subtraction algorithm was used. After all particles have been localized, a background correction of the two images was performed. The particles of interest were selected, and for those particles, the final $\mathrm{r} / \mathrm{g}$ is subtracted from the initial. Then limits are set $(0.015<\Delta \mathrm{r} / \mathrm{g}<0.1)$ and only those particles are counted that experienced a change of the $\mathrm{r} / \mathrm{g}$ within those limits.

\begin{tabular}{|l|l|l|l|l|l|l|l|l|}
\hline Concentration & & & & & & & & \\
Goat IgG (M) & $10^{-6}$ & $8 * 10^{-7}$ & $4 * 10^{-7}$ & $1 * 10^{-7}$ & $8 * 10^{-8}$ & $4 * 10^{-8}$ & $1 * 10^{-8}$ & $1 * 10^{-9}$ \\
\hline Concentration & & & & & & & & \\
\hline
\end{tabular}

Table 3-4The concentrations of target molecules used in the protein immunoassay

The experiment was conducted as follows: 
- The sensing surfaces were washed with PBS buffer for 30 minutes (flow rate=14 $\mu \mathrm{l} / \mathrm{min})$

- The target was incubated for 1 hour in a flow regime (flow rate $=14 \mu \mathrm{l} / \mathrm{min}$ )

- The excess was washed for 10 minutes with PBS buffer (flow rate=14 $\mu \mathrm{l} / \mathrm{min}$ )

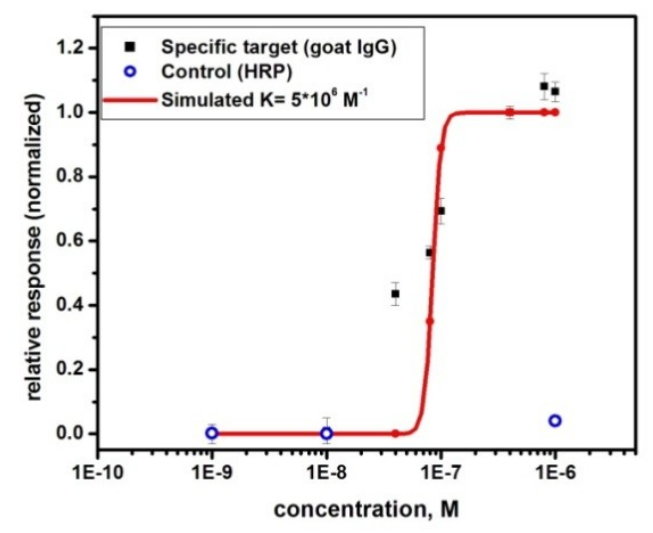

Figure 3-28 The response of multiple individual nanoparticles after the incubation with various concentrations of analyte (squares) or control (circles). The response was normalized for the comparison of the experimental data with the Monte Carlo simulation binding model.

The results of these experiments are presented in Figure 3-28. Apart from one outlier, present at $4 * 10^{-8} \mathrm{M}$, the response to the binding of specific target shows saturation at high concentrations and was followed by a steep descent and low response at low concentrations. A rapid decrease of the number of the nanoparticles that changed colour was observed around $4 * 10^{-8} \mathrm{M}$. When a non-specific target was incubated, the response was low, even at high concentration suggesting that the binding between $\alpha$-goat and goat $\mathrm{IgG}$ molecules is highly specific. The low inflection point determines the LOD of the system. For this type of assay, we estimate the LOD at around $40 \mathrm{nM}$.

The Monte Carlo model, explained in detail in Chapter 2, was used to estimate the affinity constant $\mathrm{K}$ of the binding of the $\alpha$-goat and goat IgG. From the comparison between the experimental and theoretical data, we conclude that $\mathrm{K}$ is around $5^{*} 10^{6} \mathrm{M}^{-1}$, which is a reasonable value for protein binding assays. 


\section{Comparison between two detection methods: colour camera and two monochrome camera with filters}

Until now, we have characterized and proved the proof of principle using colour camera as a detector for immunoassays. Here we have determined the characteristics that make the colour camera suitable for binding detections: sensitivity to spectral changes. A pertinent question would be to find out if this detection approach has the best sensitivity to spectral shifts.

This section is dedicated to a comparison of the signal-to-noise ratio (SNR) and the spectral shift sensitivity between the above-mentioned detection method and an alternative detection scheme.

The current setup uses a colour camera that measures the spectral changes of one nanoparticle by monitoring the ratio between the red and the green channel. Until now, we have determined that the colour CCD camera, which has a fixed RGB filter built in, is able to detect one nm spectral shifts. In order to increase the response of the camera to smaller spectral shifts one could use custom colour filters with a steeper response.

(a)

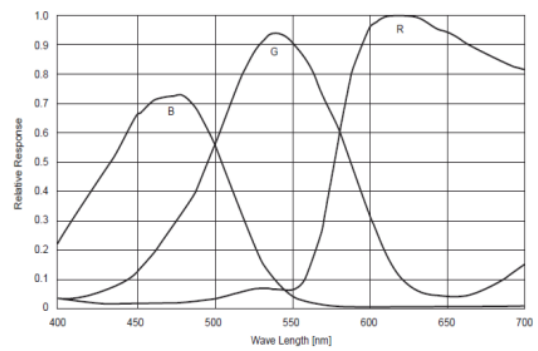

(b)

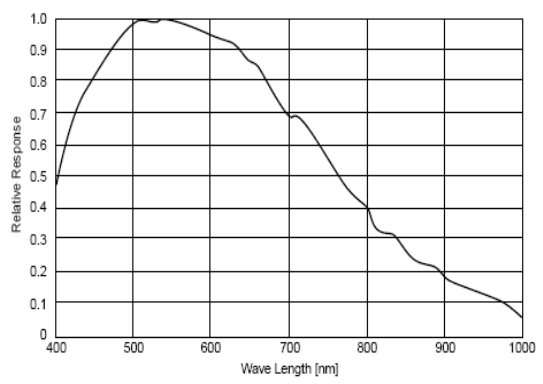

Figure 3-29 The relative spectral response of the CCD chip (ICX285AL) when a (a) colour filter (QEmax $\sim 58 \%$ ) is present or (b) absent (QEmax $\sim 65 \%$ )

Figure 3-29 shows the relative response of the same CCD chip; the left side has fixed colour filters and the right has no filters added. In addition, we mention that the inclusion of filters reduces the maximum quantum yield from $65 \%$ to $58 \%$. 
In order to use the same analysis principle, of measuring the colour change as a response to spectral shifts, in the new detection approach, we use a 50\% $\mathrm{T} / 50 \% \mathrm{R}$ beamsplitter to send the image from the sample to two identical monochrome cameras, each with a specific colour filter. Camera 1 receives information only from the red region of the spectrum while camera 2 receives its information from the green region. The two resulting images are overlapped into one RG coloured image and the red and the green channel are analyzed. Using the custom filters in front of two monochrome cameras, we expect:

- $\quad$ Large tunability of the $\mathrm{R} / \mathrm{G}$ response;

- High dynamic range;

- Spatial true colour imaging without predefinite colour patterning;

- An improved SNR due to the larger sensitivity of the monochrome camera;

All these aspects should result in a better sensitivity to spectral shifts (decrease in the minimum detectable spectral shift). A series of band pass filters were purchased for the 520-560 nm spectral range. Figure 3-30 illustrates the spectral response of several filters for the 480-60 0nm spectral range.

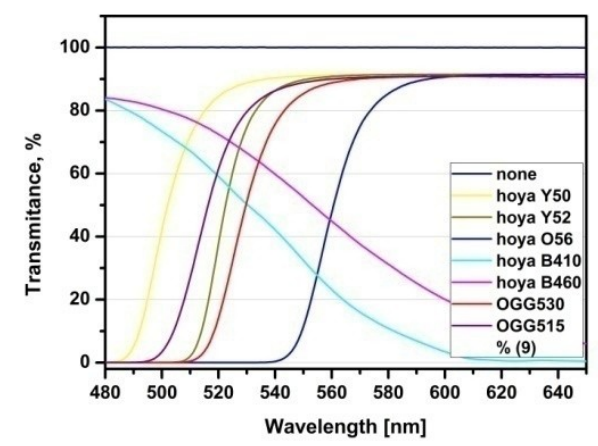

Figure 3-30 the spectral response of the selected filters in the $480-600 \mathrm{~nm}$ region.

A series of experiments were designed to measure the response to a spectral change of both detection methods. As a model for the monochrome camera, we selected the Basler camera (A102f).First, the spectrum of a gold nanoparticle was measured using the fibre spectrometer. The spectral response of each channel, respectively filters, can be calculated back by convoluting the spectral response of the channel with the measured 
spectrum of the Au nanoparticle. The spectral response of each sensor, respectively channel, is given by the quantum efficiency over the covered spectral range. . Figure 3-30 depicts the calculated responses of the red/ green channel (a) and two selected filters (b). The calculated spectral response $(r(\lambda))$ is given by:

$$
r(\lambda)=\sum_{k=525}^{575} p(\lambda-\kappa) f(\lambda)
$$

Where $p(\lambda)$ is the measured spectrum of the nanoparticle and $f(\lambda)$ is the measured transmission of the color filter.

The calculated transmission efficiencies of the Red and Green channels are $17.5 \%$ and 39.1\%respectively. For the monochrome camera B410 filter $\mathrm{T}=15.8 \%$ and $\mathrm{O} 54$ filter $\mathrm{T}=44 \%$.

a)

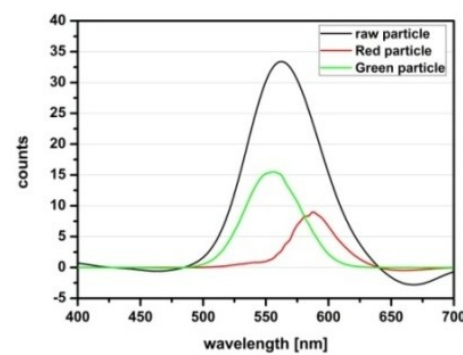

Figure 3-31 The calculated spectral response of the (a) colour camera and (b) two filters when the spectrum of a single nanoparticle is measured.

Secondly, we simulated a spectral shift of one $\mathrm{nm}$ in the 525-575nm (Figure 332a) spectral range and measured the response on each channel, respectively the selected filters. The results are depicted in Figure 3-32b. Some of the filters show a steeper response (O54, O56, B410, B460) while others show a flattening of the response.

The green channel of the colour camera shows a flat signal at lower wavelengths (525-540 nm) suggesting that at that range the response to spectral shifts, in that region, falls on the red channel. In the same region, the red channel has a low counting rate, which adds more noise into the signal. For the best sensitivity a steep linear response to 
wavelength changes and high counting rates are needed; thus from the custom filters B460 and $\mathrm{O} 54$ show promise of a better sensitivity.

(a)

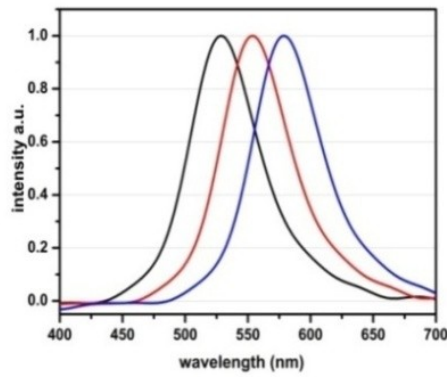

(b)

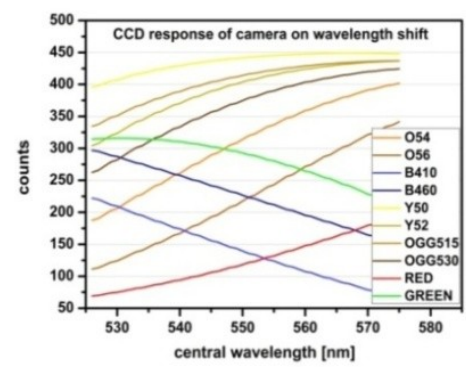

Figure 3-32 (a) The shifting of the spectral maximum of an individual gold nanoparticle from 525 to $575 \mathrm{~nm}$ in steps of $1 \mathrm{~nm}$. (b) The response of the filters and the Red and Green channel on the selected spectral range.

Next, we calculate the signal to noise ratio (SNR) for the selected filters and for the red and green channels of the colour camera. For the best response, the sensors should be illuminated at $80 \%$ of their full well capacity. For the colour camera, only one channel can fulfil this requirement, as the exposure times on each channel cannot be independently set. On the other hand, on the two monochrome +added filters setup, the exposure times are different such that each sensor delivers the maximum response. To evaluate the noise levels of the Basler CCD chip, the following formula is used:

$$
S N R=\frac{\eta \mu_{p}}{\sqrt{\eta \mu_{p}+\left({\sigma_{r}}^{2}+{\sigma_{f}}^{2}+\sigma_{o}{ }^{2}\right)+S_{g}{ }^{2} \cdot \eta \mu_{p}{ }^{2}}}
$$

where $\eta \mu_{p}=18,000 \mathrm{e}$ is the full well capacity of the sensor, $\sigma_{r}{ }^{2}=9 \mathrm{e}$, random noise, $\sigma_{o}{ }^{2}$ $=2.5 \mathrm{e}$ is the offset noise and $S_{g}=0.7 \%$ is the Spatial gain noise. Figure 3-33depicts the noise levels of the CCD used that show that the temporal noise is shot noise limited. In practice, the spatial noise was limited to a SNR of 97. 


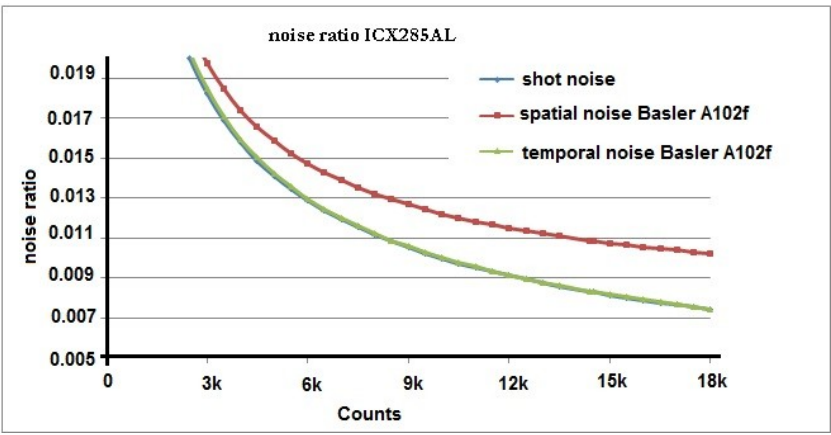

Figure 3-33 The noise levels of the CCD chip

The uncertainty in calculating the $\mathrm{R} / \mathrm{G}$ ratio, respectively $\mathrm{O} 54 / \mathrm{B} 460$ ratio is given by the accuracy in measuring the intensity levels. This was estimated to $2.19 \%$ for the colour camera and $1.54 \%$ for the custom filter setup.

(a)

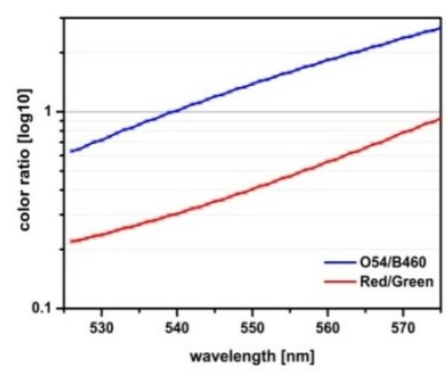

(b)

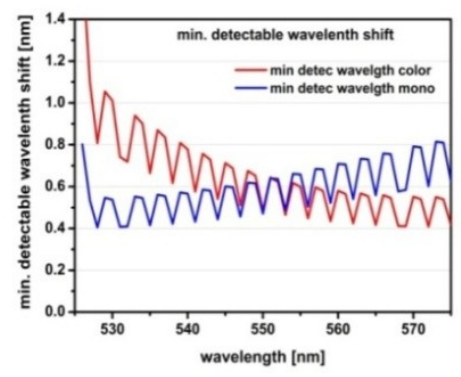

Figure 3-34 (a) The response of the two detection systems to spectral shifts in the $525-575 \mathrm{~nm}$ spectral range. (b) The minimum detectable wavelength shifts of the two detection systems (the ripple effect on the data is given by the simulated spectrum of the nanoparticle).

We calculated the responses of the colour camera $R / G$ and $O 54 / B 460$ ratios over the 525-575 nm spectral range taking into accounts the noise levels (Figure 3-34a). The results show that the two systems behave quite similarly as they show the same slope. The O54/ B460 response shows a small curvature, which is the result of the variance in the count rates (given by the different exposure times). The noise levels are also comparable.

In determining the minimum detectable wavelength shift, we calculated the ratio between the change in ratio at wavelength $\lambda$, divided over the error produced by noise at $\lambda$ 
$\overline{\left(\frac{d R / G}{d \lambda}\right) \text {. The results are depicted in Figure 3-34b. In the region } 525-535 \mathrm{~nm} \text {, the colour }}$ camera shows lower spectral shift sensitivity, as a direct result of the low response change of the green channel and low signal of the red channel. The sensitivity increases at larger wavelengths, being able to detect $0.5 \mathrm{~nm}$ shifts. The particles used in our experiments $(60$ $\mathrm{nm}$ or $80 \mathrm{~nm}$ Au particles) have PP at wavelengths higher than $540 \mathrm{~nm}$, which is exactly where the colour camera has the maximum sensitivity. In contrast, the other detection system is more accurate in the $525-535 \mathrm{~nm}$ spectral range and has a tendency in decreasing its accuracy for larger wavelengths. This could suggest that smaller particles can be suitable for this approach. However, smaller particles have a smaller scattering cross section that makes them more difficult to visualize.

In conclusion, using custom filters over the present colour camera is not as advantageous as expected. The shape and the width of the spectrum of individual gold nanoparticle can be the reason for this limitation.

For this type of applications, the results of this study shows that the colour camera approach has net advantages over the other detection approach.

\section{Conclusions}

In summary, this work shows the feasibility of using the colour camera for the measuring of a kinetic process and its quantification.

By proving its feasibility, the use of a colour camera shows a clear advantage over the current spectroscopic detection methods, as it can perform a parallel measurement of the optical properties of all nanoparticles present in the FOV. Additionally, we proved that a concentration as low as $40 \mathrm{nM}$ can be measured. However, the sensitivity of our method using the current type of assay is not sufficient for many practical sensor applications. To this end, the introduction of an alternative type of assay, based upon the wavelength shift of approaching nanoparticles, as discussed in Chapter 2, seems more adequate [15],[32], [33]. In addition, the concept of the detection method presented in this chapter provides an outlook to its use in wavelength multiplexing sensing. 


\section{Appendix}

\section{- Antibody modification}

As thiols exhibit a high affinity to a gold surface, this group was conjugated to the anti-goat antibody. To this end a $100 \mu \mathrm{l} \mathrm{Ab}$ solution $\left(6.7 \cdot 10^{-10} \mathrm{M}\right)$ was mixed with $100 \mu \mathrm{l}$ 2-iminothiolane solution $\left(7.3 \cdot 10^{-7} \mathrm{M}\right)$ in $700 \mu \mathrm{l}$ PBS buffer $\mathrm{pH} 8.7$ and was incubated for 120 min under shaking at $4^{\circ} \mathrm{C}$ and at $250 \mathrm{RPM}$. The excess of unbound 2-iminothiolane was removed by ultra-filtration. The immunoreactivity of this conjugate towards the antibiotin protein turned out to remain intact, as was apparent from a series of immunoprecipitation experiments (data not shown).

\section{- $\quad$ Preparation of gold nanoparticle conjugates}

$10 \% \mathrm{v} / \mathrm{v}$ of 2,5-dioxopyrrolidin-1-yl 26-mercapto-3,6,9,12,15-pentaoxahexacosyl carbonate (prochimia, TH013) in acetonitrile (p.a., Merck), sonicated for 2 min and the freshly prepared solution was added to citrate capped AuNP colloids (80 nm diameter, $2 * 10^{-14} \mathrm{M}$ ) to yield a final concentration of $0.5 \% \mathrm{v} / \mathrm{v}$. The mixture was left to react for 10 min $\left(20 \mathrm{rpm}, 21^{\circ} \mathrm{C}\right)$. Immediately afterwards it was mixed with $5 \mu \mathrm{M}$ solutions of rabbit antigoat polyclonal antibody ( $\alpha$-goat Ab) (Sigma Aldrich, G4018), goat IgG antibody (Sigma Aldrich, B3640) or horse radish peroxidise (HRP) (VI, Sigma Aldrich, P6782) in 50 $\mathrm{mM}$ borate buffer ( $\mathrm{pH}$ 9). The obtained molar ratios for each protein were 1:50, 1:500 and 1:5000 AuNP: protein while keeping the absolute sample volume constant. The reaction mixtures were incubated for $48-72 \mathrm{~h}\left(20 \mathrm{rpm}, 4^{\circ} \mathrm{C}\right)$. The resulted conjugates were washed three times by centrifuging ( $40 \mathrm{~nm}$ particles: $9300 \mathrm{xg}$, RT and $80 \mathrm{~nm}$ particles $2300 \mathrm{xg}, \mathrm{RT}$ ), decanting and resuspending in $5 \mathrm{mM}$ borate buffer ( $\mathrm{pH} 9$ ) in the first 2 steps and in phosphate buffered saline (PBS) in the last step. 


\section{- Surface modification}

Prior to any modification of the glass slides a cleaning procedure was used (Piranha solution $\left(1 \mathrm{H}_{2} \mathrm{O}_{2}: 3 \mathrm{H}_{2} \mathrm{SO}_{4}(>96 \%)\right.$ ) at $95{ }^{\circ} \mathrm{C}$ for $15 \mathrm{~min}$ ). Immediately after cleaning, the slides were washed with MilliQ water (MQ) under sonication for 5 minutes. Then the glass slides were dipped in a solution of 10\% 3-Aminopropyl Triethoxysilane (APTES) for 10 minutes and then 5 times washed with MQ water and sonicated each time for 1 minute. Through this procedure, we ensure that the excess of APTES is removed and the layer of the coating is uniformly distributed over the glass surface. Subsequently, the surfaces were baked for 2 hours in an oven at $100^{\circ} \mathrm{C}$. This procedure removed all water residues from the surface.

\section{- Surface modification with bare gold nanoparticles}

After baking, the slides were dipped in a gold solution of $1.4 \cdot 10^{9}$ part $/ \mathrm{ml}$ for 5 minutes, resulting in nanoparticle-covered substrate, where the particles had an average distance of $\sim 10 \mu \mathrm{m}$. The interaction of the nanoparticles and the APTES layer acts through the APTES amino groups and is of electrostatic nature. Although it is a weak interaction, it is sufficient to keep the nanoparticles on the surface both in high flow rates and the high voltages required for Scanning Electron Microscopy (SEM) investigations.

- Surface modification with functionalized gold nanoparticles

A different immobilization approach is utilized when functionalized gold nanoparticles are employed. This method ensures a better immobilization of the conjugates particles on the surface (covalent binding is used in this immobilization procedure). The course of action consists of two steps: surface passivation (surface coverage with a chemical that does not allow the non-specific interaction of target molecules to the surface) and the covalent binding of the functionalized nanoparticles to the resulted surface. First, the APTES surface is exposed to a mixture of NHS/EDC for 10 minutes in order to activate the amino groups of the APTES layer. The surface is then rinsed abundantly with MQ water and immediately after a solution of bovine serum albumin $(B S A)\left(10^{-7} \mathrm{M}\right)$ is added. The BSA was left to react with the exposed amino 
groups of the APTES for 3 hours. We expect a fully covered surface as excess of BSA was used. The passivated surface was again exposed to the same NHS/EDC mixture for another 10 minutes to activate the amino groups of the BSA. The surface was washed plentifully with MQ water. The functionalized nanoparticles $\left(\alpha\right.$-goat- $\left.A u^{80}, 10^{-10} \mathrm{M}\right)$ are incubated for 10 minutes and the excess was washed away with borate buffer. 


\section{References}

[1] S. Schultz, D.R. Smith, J.J. Mock, and D.A. Schultz, "Single-target molecule detection with nonbleaching multicolor optical immunolabels ," Proceedings of the National Academy of Sciences , vol. 97 , Feb. 2000, pp. 996-1001.

[2] N. Nath and A. Chilkoti, "A Colorimetric Gold Nanoparticle Sensor To Interrogate Biomolecular Interactions in Real Time on a Surface," Analytical Chemistry, vol. 74, Dec. 2001, pp. 504-509.

[3] N. Nath and A. Chilkoti, "Label Free Colorimetric Biosensing Using Nanoparticles," Journal of Fluorescence, vol. 14, 2004, pp. 377-389.

[4] J. Yguerabide and E.E. Yguerabide, "Light-Scattering Submicroscopic Particles as Highly Fluorescent Analogs and Their Use as Tracer Labels in Clinical and Biological Applications: I. Theory," Analytical Biochemistry, vol. 262, Sep. 1998, pp. 137-156.

[5] W. Fritzsche and T.A. Taton, "Metal nanoparticles as labels for heterogeneous, chip-based DNA detection," Nanotechnology, vol. 14, 2003, p. R63.

[6] L.A. Trachuk, A.G. Melnikov, and N.G. Khlebtsov, "Dependence of the optical properties of metal nanoparticles on the external dielectric medium: effects of the particle size, shape, and structure," SPIE Proceedings, vol. 5829, 2005, pp. 127-137.

[7] G. Raschke, S. Kowarik, T. Franzl, C. Sönnichsen, T.A. Klar, J. Feldmann, A. Nichtl, and K. Kürzinger, "Biomolecular Recognition Based on Single Gold Nanoparticle Light Scattering," Nano Letters, vol. 3, May. 2003, pp. 935-938.

[8] C. Noguez, "Surface Plasmons on Metal Nanoparticles: The Influence of Shape and Physical Environment," The Journal of Physical Chemistry C, vol. 111, Feb. 2007, pp. 38063819.

[9] T. Okamoto, I. Yamaguchi, and T. Kobayashi, "Local plasmon sensor with gold colloid monolayers deposited upon glass substrates," Opt. Lett., vol. 25, Mar. 2000, pp. 372-374.

[10] J.N. Anker, W.P. Hall, O. Lyandres, N.C. Shah, J. Zhao, and R.P. Van Duyne, "Biosensing with plasmonic nanosensors," Nat Mater, vol. 7, Jun. 2008, pp. 442-453.

[11] P.K. Jain, K.S. Lee, I.H. El-Sayed, and M.A. El-Sayed, "Calculated Absorption and Scattering Properties of Gold Nanoparticles of Different Size, Shape, and Composition: Applications in Biological Imaging and Biomedicine," The Journal of Physical Chemistry B, vol. 110, Mar. 2006, pp. 7238-7248. 
[12] C.R. Yonzon, D.A. Stuart, X. Zhang, A.D. McFarland, C.L. Haynes, and R.P. Van Duyne, "Towards advanced chemical and biological nanosensors--An overview," Talanta, vol. 67, Sep. 2005, pp. 438-448.

[13] A.J. Haes, L. Chang, W.L. Klein, and R.P. Van Duyne, "Detection of a Biomarker for Alzheimer's Disease from Synthetic and Clinical Samples Using a Nanoscale Optical Biosensor," Journal of the American Chemical Society, vol. 127, Jan. 2005, pp. 2264-2271.

[14] A.J. Haes and R.P. Van Duyne, "A Nanoscale Optical Biosensor: Sensitivity and Selectivity of an Approach Based on the Localized Surface Plasmon Resonance Spectroscopy of Triangular Silver Nanoparticles," Journal of the American Chemical Society, vol. 124, Aug. 2002, pp. 10596-10604.

[15] T. Sannomiya, C. Hafner, and J. Voros, "In situ Sensing of Single Binding Events by Localized Surface Plasmon Resonance,” Nano Letters, vol. 8, Sep. 2008, pp. 3450-3455.

[16] C.L. Baciu, J. Becker, A. Janshoff, and C. Sönnichsen, "Protein-Membrane Interaction Probed by Single Plasmonic Nanoparticles,” Nano Letters, vol. 8, May. 2008, pp. 1724-1728.

[17] "http://www.ruf.rice.edu/ bioslabs/methods/microscopy/dfield.html."

[18] K.B. Wolf and G. Krotzsch, "Geometry and dynamics in refracting systems," European Journal of Physics, vol. 16, 1995, p. 14.

[19] “Menzel Glaser coverslip, http://www.menzel.de/Cover_Slips.675.0.html?L=1.”

[20] "BBI gold, http://www.bbigold.com/Gold_Colloid_12.aspx."

[21] "http://www.smt.zeiss.com/orion."

[22] D. Millan, “http://blog.damiles.com/2009/03/the-basics-of-background-substraction/."

[23] H. Palus, "Representations of color images in different color spaces," The Colour Image Processing Handbook, 2000.

[24] H. Ailisto and T. Piironen, "Evaluation of color representation methods in a practical vision system," Scand. Conf. Image Analysis (SCLA), 1987.

[25] I. Andreadis and P. Tsalides, "Coloured object recognition using invariant spectral features," Journal of Intelligent \& Robotic Systems, vol. 13, pp. 93-106.

[26] P. Lee, R.M. van den Berg, S. Lam, A.F. Gazdar, K. Grunberg, A. McWilliams, J. LeRiche, P.E. Postmus, and T.G. Sutedja, "Color Fluorescence Ratio for Detection of Bronchial Dysplasia and Carcinoma In situ," Clinical Cancer Research, vol. 15, 2009, pp. 4700-4705.

[27] M.W. Davidson, "Concepts and formulas in microscopy: Resolution." 
[28] A.D. McFarland and R.P. Van Duyne, "Single Silver Nanoparticles as Real-Time Optical Sensors with Zeptomole Sensitivity," Nano Letters, vol. 3, Jul. 2003, pp. 1057-1062.

[29] A. Zimmermann, "Shifting colors," Imaging \& Microscopy, vol. 4, 2002, pp. 62-63.

[30] H. Ouyang, C.C. Striemer, and P.M. Fauchet, "Quantitative analysis of the sensitivity of porous silicon optical biosensors," Applied Physics Letters, vol. 88, Apr. 2006, pp. 163103 163108.

[31] "http://www.delta-technologies.com/Products.asp?C=13."

[32] W. Rechberger, A. Hohenau, A. Leitner, J.R. Krenn, B. Lamprecht, and F.R. Aussenegg, "Optical properties of two interacting gold nanoparticles," Optics Communications, vol. 220, 2003, pp. 137-141.

[33] P.K. Jain, S. Eustis, and M.A. El-Sayed, "Plasmon Coupling in Nanorod Assemblies: Optical Absorption, Discrete Dipole Approximation Simulation, and Exciton-Coupling Model," The Journal of Physical Chemistry B, vol. 110, Aug. 2006, pp. 18243-18253. 


\section{Chapter 4}

\section{Detection of individual binding events in a direct immunoassay}

$\boldsymbol{A b s t r a c t}^{4}$ : A new (generic) sensor platform is introduced using gold nanoparticle probes in microfluidic cells in colorimetric darkfield microscopy enabling the simultaneous sensing of hundreds of binding events of individual particles. The resulting timedependent colorimetric data showed that binding events of individual particles could be discerned for about $40 \%$ of the available binding sites while reference data showed less than $0.05 \%$ binding. Endpoint SEM images of the sample and reference binding couples corroborate our findings.

Keywords: gold nanoparticles, colorimetric dark field microscopy, immuno sensor, parallel detection, affinity constant

${ }^{4}$ This chapter has been published as "Immunosensing by Colorimetric Darkfield Microscopy of Individual Gold Nanoparticle-Conjugates" , Felicia Ungureanu, Dorothee Wasserberg, Remco Verdoold, Rob P.H. Kooyman, Sensors and Actuators B: Chemical (2010), Volume: 150, Issue: 2, pp 529-536 


\section{Introduction}

The majority of conventional optical biosensing approaches rely on the measurement of a signal originating from an ensemble of molecules. However, these methods either lack sufficient specificity and sensitivity (e.g. SPR) or are time consuming and require trained personnel (e.g. ELISA) $[1,2]$. A new biosensing approach using noble metal nanoparticles as sensors may overcome these limitations [3-7].

The advantage of using noble metal nanoparticles arises from their Localized Surface Plasmon Resonance (LSPR) modes that, when excited, strongly enhance the nanoparticle's absorption and scattering cross-sections. Thus, individual nanoparticles become "visible" in darkfield microscopy $[8,9]$. The resonance frequency of the LSPR modes depends on the nanoparticle's size, shape, composition and surrounding medium. Of particular interest for biosensing applications is the dependence of the plasmon peak on the local refractive index change of the surrounding medium [10]. The local refractive index is closely related to the number of molecules adsorbed on the surface of a nanoparticle. The potentially greatest advantage of using individual nanoparticles as a sensor platform over ensemble measuring techniques, like SPR [11], colorimetric methods [12-14] or ELISA is the possibility of detecting very small numbers of molecules [15-17]. However, even in this sensing strategy single molecule detection is hard to achieve. Especially when small molecules bind to the nanoparticle surface they cause a very low refractive index change making them hard to detect [18]. Thus, an amplification of the sensing system is required. For example, such amplification can be obtained using one immobilized noble metal nanoparticle conjugated with target-specific receptors and a second such particle labelled with the target molecules. The binding between target and receptor molecules will bring the two particles in close proximity to each other causing a very large effect on the plasmon resonance conditions. Previous studies regarding the influence of the inter-particle distance on the optical properties predict that under the proper conditions, right interparticle distance and size of the particles, even a single binding event can be easily detected [19-23]. However, in many sensor applications it is not an absolute number of particles that is of interest but a concentration of target 
4. Detection of individual binding events in a direct immunoassay

molecules in a solution. Using an equilibrium surface binding reaction (as for example described by the Langmuir isotherm) the fraction of occupied binding sites of the total number of binding sites needs to be determined. Thus, in order to detect very low concentrations $(<1 \mathrm{pM})$ extensive parallelized detection of at least $10^{4}$ individual nanoparticles is needed when assuming an affinity constant $\mathrm{K}_{\mathrm{A}}$ in the order of $10^{8} \mathrm{M}^{-1}$.

For plasmon based optical nanosensors a common detection strategy to determine the LSPR peak position is to measure the extinction/scattering spectra of individual nanoparticles. This can be done by either using a spectrometer that measures the spectrum of a single particle [24-26], or by using filters (like LCTF or a set of AOTF filters); the former allowing for the simultaneous collection of spectra of only a limited number of different particles [27, 28]. Though precise, these methods are time consuming, expensive and vast parallelization cannot be easily achieved.

To overcome these difficulties Gunnarsson et al. [38] have use specific ssDNA labelled vesicles on a substrate covered with complementary strands. This approach, however, has several disadvantages: the surface coverage (and thus, the quantification) is problematic and the inability to distinguish between specific and non-specific binding represents a non-negligible drawback.

Here, we propose an alternative detection method by using a colour camera to detect binding events associated with single nanoparticles for a large number of particles simultaneously in an immunoassay. With this system the change of the color of a nanoparticle indicates when a modification of the nanoparticle's "binding state" occurs. In addition, in the case of labelled assays the spectral resolution of the camera is large enough to observe binding associated with a single particle. This aspect is demonstrated with calculations and the calibration of the camera. For ease of representation we introduce the parameter $\mathrm{r} / \mathrm{g}$, defined as the ratio between the values of the intensity of the red and the green channels of the camera, which has been shown to be directly proportional to the spectral changes of a nanoparticle [29, 30]. Although the time resolution of the camera is relatively low, it is not essential for the monitoring of a binding process because typical time constants to obtain chemical equilibrium, for the system considered here, are in the orders of tens of minutes $[36,37]$. The immediate advantages of this method are: 
4. Detection of individual binding events in a direct immunoassay

- the possibility of parallel detection and monitoring in time of hundreds to thousands of individual nanoparticles; it follows that very low concentrations become detectable and simple quantification may become feasible.

- the possibility of wavelength multiplexing by using multiple types of gold nanoparticles that can be discriminated by their unique LSPR characteristics.

- the fact that the method is generic in so far as it can be used in any protein-protein binding reaction and that no dedicated instrument is required; a standard darkfield objective and a colour camera is all that is needed.

Thus, we here report the simultaneous detection of a large number of binding events associated with single nanoparticles in an immuno assay using the effect of coupling two particles to each other using a darkfield (DF) microscope for detection.

\section{Materials and methods}

\subsection{Calculations}

The binding of a single nanoparticle can be observed if for parameters such as size of the particles involved in the assay and inter-particle distance, the appropriate values are chosen.

For good visibility in our DF setup we chose gold nanoparticles (AuNPs) of 80 $\mathrm{nm}$ diameter, as the first of the nanoparticles.

To select the size of the second particle involved in the assay we conducted simulations using DDSCAT software by Draine and Flatau [31]. This program uses the discrete dipole approximation method to iteratively calculate the optical properties of nanoparticles. The particle under investigation is discretized into small dipoles and the electromagnetic field scattered by the particle is calculated from the interaction between all constituent dipoles and the incident electromagnetic field. In simulations the bulk dielectric function of gold was used [32] and water with a refractive index of 1.33 was 
4. Detection of individual binding events in a direct immunoassay

considered as surrounding medium. This software has been used successfully before for the study of optical properties of metallic nanoparticles [33, 34].

In particular, we considered one particle of $80 \mathrm{~nm}$ in diameter, $\mathrm{p} 1$, in the centre of the coordinate system and set out to determine the optimal characteristics, with regard to size and distance, of a second particle, p2, in order to maximize the optical response upon the particles' approach (Figure 4-1).

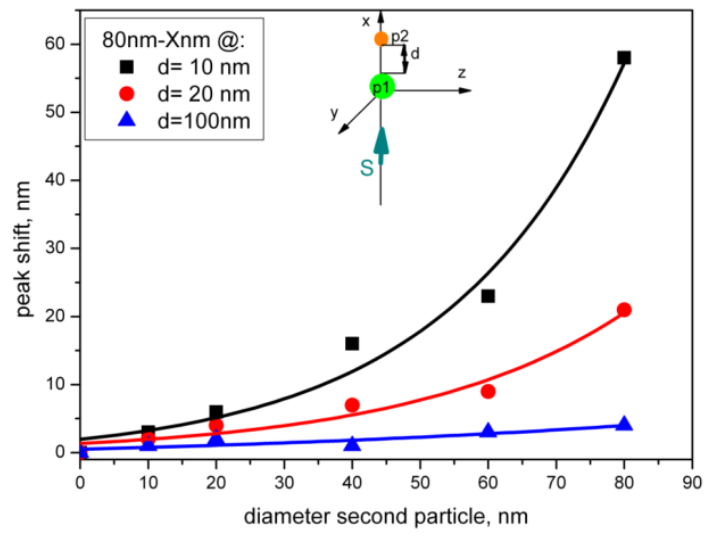

Figure 4-1 Plasmon resonance peak shifts of an $80 \mathrm{~nm}$ Au particle, p1, coupled to a second particle, p2, as a function of the diameter of p2 for $10 \mathrm{~nm}$ (squares), $20 \mathrm{~nm}$ (spheres) and $100 \mathrm{~nm}$ (triangles) inter-particle distances, d. The inset shows a schematic drawing to determine the parameter $\mathrm{d}, \mathrm{p} 1, \mathrm{p} 2$, as well as the direction of the incident light, $\mathrm{S}$, with regard to the coordinate system.

The results of the simulations, at a constant diameter of particle, p1, of $80 \mathrm{~nm}$ indicate that shifts become larger with larger particles, p2, and shorter inter-particle distance, $\mathrm{d}$. However, distance, $\mathrm{d}$, is dictated by the size of the proteins used in the assay. Here, anti-goat antibody ( $\alpha$ goatAb) and a goat $\operatorname{IgG}$ were used, each with an approximate average diameter of $10 \mathrm{~nm}$; the average interparticle distance is therefore estimated to be $20 \mathrm{~nm}$.

In a DF microscope, the use of secondary particles, p2, much smaller than the first type of particles, p1, is preferable as they are barely visible compared to the larger particles, p1, resulting in a low additional background signal. In addition, the size 
4. Detection of individual binding events in a direct immunoassay

difference between the two particles forming the complex is advantageous for other methods like Scanning Electron Microscopy (SEM) enabling us to distinguish the two types of nanoparticles. Thus, from our simulations and from the above-mentioned selection criteria we chose $40 \mathrm{~nm}$ Au particles as a good compromise between the spectral shift upon binding and induced background signal. From simulations only one peak shift (without any further changes of shape) of at least $5 \mathrm{~nm}$ is to be expected upon binding of a single $40 \mathrm{~nm}$ diameter $\mathrm{AuNP}\left(\mathrm{Au}^{40}\right)$ to an $80 \mathrm{~nm}$ diameter $\mathrm{AuNP}\left(\mathrm{Au}^{80}\right)$ at a distance (determined by the immuno couple) of an average of $20 \mathrm{~nm}$ which can easily be detected with our system [30]. It should be noted that the localization of the bound particle p2 on the surface of the particle p1 as well as the number of bound particles has also been shown to influence the resulting wavelength shift, which may result in a variety of differently sized shifts [25].

\subsection{Experimental section}

Two sets of binding assays were conducted (Figure 4-2): (1) as the sample: the binding of goat $\operatorname{IgG}$ functionalized $40 \mathrm{~nm}$ Au particles (goat IgG-Au ${ }^{40}$ ) to rabbit anti-goat antibody functionalized $80 \mathrm{~nm}$ Au particles $\left(\alpha g o a t-\mathrm{Au}^{80}\right)$ and (2) as the reference: the lack of binding of Horseradish Peroxidase functionalized $40 \mathrm{~nm}$ Au particles $\left(\mathrm{HRP}-\mathrm{Au}^{40}\right)$ to agoat- $\mathrm{Au}^{80}$.

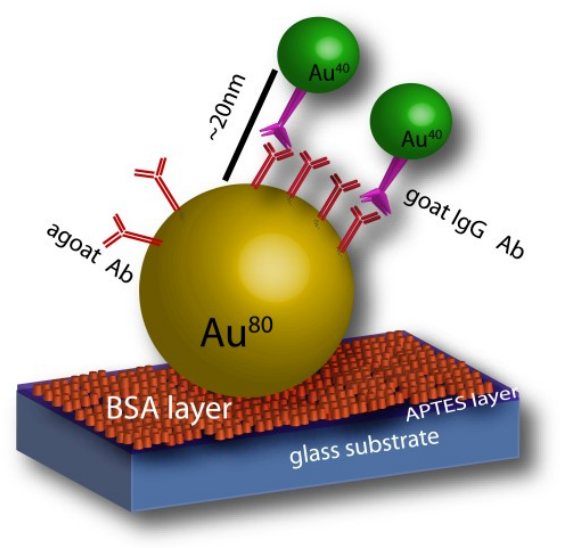

Figure 4-2 Schematic representation of the binding assay conducted here: rabbit anti-goat antibody functionalized $80 \mathrm{~nm}$ Au nanoparticles ( $\left(\right.$ goat $\left.-\mathrm{Au}^{80}\right)$ immobilized on a BSA coated glass substrate 
4. Detection of individual binding events in a direct immunoassay

binding goat IgG functionalized $40 \mathrm{~nm}$ Au nanoparticles (goat $\mathrm{IgG}-\mathrm{Au}^{40}$ ) during incubation from solution. In the reference experiment goat $\operatorname{IgG}$ has been replaced by non-binding horseradish peroxidase (HRP).

\subsubsection{Preparation of gold nanoparticle (AuNP) conjugates}

All chemicals and proteins were used as purchased, without further purification. Water used for the preparation of buffers, solutions and for washing was exclusively of milliQ quality.

$10 \% \mathrm{v} / \mathrm{v}$ of 2,5-dioxopyrrolidin-1-yl 26-mercapto-3,6,9,12,15-pentaoxahexacosyl carbonate (prochimia, TH013) in acetonitrile (p.a., Merck), sonicated for 2 min and the freshly prepared solution was added to citrate capped AuNP colloids (either $80 \mathrm{~nm}$ or 40 nm diameter, $2 * 10^{-14}$ and $2 * 10^{-13} \mathrm{M}$, respectively, BBI) to yield a final concentration of $0.5 \% \mathrm{v} / \mathrm{v}$. The mixture was left to react for $10 \mathrm{~min}\left(20 \mathrm{rpm}, 21^{\circ} \mathrm{C}\right)$ and immediately afterwards mixed with $5 \mu \mathrm{M}$ solutions of rabbit antigoat polyclonal antibody (Sigma Aldrich, G4018), goat IgG antibody (Sigma Aldrich, B3640) or horse radish peroxidase (VI, Sigma Aldrich, P6782) in $50 \mathrm{mM}$ borate buffer ( $\mathrm{pH}$ 9) yielding final molar ratios of 1:50, 1:500 and 1:5000 AuNP:protein for each protein while keeping the absolute sample volume constant. The reaction mixtures were incubated for a minimum of $48 \mathrm{~h}(20 \mathrm{rpm}$, $4^{\circ} \mathrm{C}$ ), then washed three times by centrifuging (40 nm particles: $9300 \mathrm{xg}$, RT and $80 \mathrm{~nm}$ particles 2300xg, RT), decanting and resuspending in $5 \mathrm{mM}$ borate buffer $(\mathrm{pH} 9)$ in the first 2 steps and in phosphate buffered saline (PBS) in the last step.

\subsubsection{DF experiment}

The $\alpha$ goat- $\mathrm{Au}^{80}$ particles were immobilized on BSA-coated glass slides and mounted on an in-house design microfluidic device. 
4. Detection of individual binding events in a direct immunoassay

Standard microscope glass slides (76 mm x $26 \mathrm{~mm}$ x $1.1 \mathrm{~mm}$, Carl Roth) were used as substrates for the immobilization of AuNP conjugates. Slides were activated by immersing them for $15 \mathrm{~min}$ in a freshly prepared 3:1 mixture of $\mathrm{H}_{2} \mathrm{SO}_{4}: \mathrm{H}_{2} \mathrm{O}_{2}$, subsequently immersed in water, rinsed thoroughly with water and used immediately for further surface modification. Slides were aminated by immersing them for $15 \mathrm{~min}$ in a $5 \% \mathrm{v} / \mathrm{v}$ solution of 3-amino propyl triethoxy silane (APTES) in EtOH (p.a., Merck). Slides were then rinsed thoroughly with water and sonicated thrice for 5 min using fresh water each time. Then, the slides were rinsed with abundant amounts of water and baked for $2 \mathrm{~h}$ at $120^{\circ} \mathrm{C}$. Slides were stored for short periods of time at $120^{\circ} \mathrm{C}$ or immersed in $\mathrm{EtOH}$ (p.a., Merck) at $4^{\circ} \mathrm{C}$ for longer periods of time.

Protein AuNP conjugates were immobilized on thus aminated slides by immersing the slides in the respective colloids: Generally, protein AuNP conjugates were diluted 10x prior to use and immobilization usually took 2-3 h. After immobilization slides were rinsed thoroughly with PBS.

The resulting AuNP-conjugate functionalized flowcell was mounted on our DF setup and an Alladin-1000 syringe pump was attached to the cell for carrying out the flow assay. An Olympus GX71 inverted microscope in reflection mode, with an MPLAN 20 x darkfield objective was used, in combination with a 75 Watt Xe lamp as a light source. The scattered light was collected by the same objective and transmitted to a colour camera (Carl Zeiss HRc , 14 bit). Images were acquired using an in-house Labview acquisition program, which allows for the automatic acquisition of multiple consecutive images and aligns the consecutive images of one experiment compensating for possible lateral shift during the course of a flow assay.

The flow assays were conducted as follows:

1. 30 minutes washing with phosphate buffered saline $(\mathrm{PBS})($ flow rate $=14 \mu \mathrm{l} / \mathrm{min})$

2. Incubation with a solution containing the $40 \mathrm{~nm}$ Au particles (goat $\mathrm{IgG}-\mathrm{Au}^{40}\left(10^{-10} \mathrm{M}\right.$ ) for the sample and HRP-Au${ }^{40}\left(10^{-10} \mathrm{M}\right)$ for the reference, respectively) for 4 hours (no flow)

3. 60 minutes washing with PBS (flow rate $=14 \mu \mathrm{l} / \mathrm{min}$ ) 
4. Detection of individual binding events in a direct immunoassay

(a)
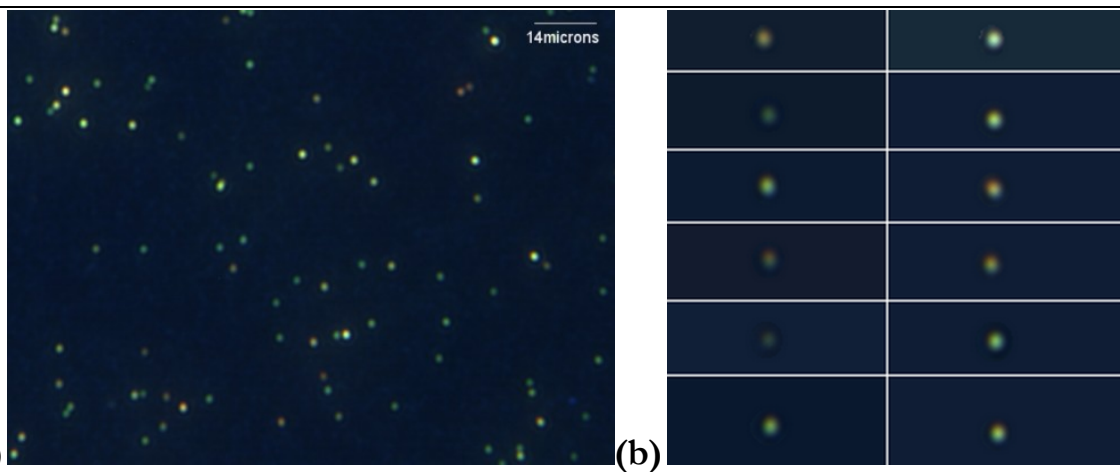

Figure 4-3 (a) Typical darkfield image of immobilized $80 \mathrm{~nm}$ diameter AuNP-conjugates of a specific field of view (FOV). (b) Some particles that show evidence of color change: left column shows the particles at the beginning while right column shows the particles at the end of the experiment.

During the study consecutive images of the nanoparticles within the field of view $\left(\mathrm{FOV}=270 \mathrm{x} 165 \mu \mathrm{m}^{2}\right.$ ) were recorded (one every minute) using the colour camera with an acquisition time of 2.5 seconds per image. Images were acquired using an in-house Labview acquisition program, which allows for the automatic acquisition of multiple consecutive images and aligns the consecutive images of one series compensating for possible lateral shift during the course of a flow assay. Figure 4-3a) shows a typical DF image while Figure 4-3b) shows some examples of particles that change colour during incubation.

\subsubsection{SEM experiments}

For scanning electron microscopy (SEM) experiments the $\alpha_{\text {goat-Au }}{ }^{80}$ particles were immobilized on an in-house manufactured gold-coated substrate.

Gold-coated glass slides (17 mm x $17 \mathrm{~mm}$ x $1.1 \mathrm{~mm}$, PGO, D263 T) were used as substrates for the immobilization of AuNPs and their conjugates for SEM measurements. Substrates were cleaned by consecutive washing steps using isopropanol, acetone, ethanol and water and dried in a flow of dry $\mathrm{N}_{2}$. Substrates were then incubated at $4{ }^{\circ} \mathrm{C}$ overnight in a humidity chamber using a $2 \mathrm{mM}$ solution of mercapto undecanoic acid (MUA, 450561 Sigma Aldrich) in a 1:1 mixture of EtOH (p.a., Merck) and water [39]. After 
4. Detection of individual binding events in a direct immunoassay

incubation the substrates were thoroughly washed with EtOH (p.a., Merck) and water and dried in a flow of dry $\mathrm{N}_{2}$. The surface was immediately covered with a freshly prepared 0.3 M solution of N-hydroxysuccinimide (NHS, 56480 Fluka) mixed with a 0.04 M solution of $N$-(3-Dimethylaminopropyl)-N'-ethylcarbodiimide (EDC, 03449 Aldrich) in water and left to incubate for $15 \mathrm{~min}$. The substrates were then briefly washed with water and PBS, immediately incubated with the appropriate protein-AuNP conjugates at $4^{\circ} \mathrm{C}$ for $2 \mathrm{~h}$ in a humidity chamber, washed with PBS and kept immersed in PBS until further use. For subsequent immuno binding experiments, substrates were immersed in a solution of the corresponding conjugate, incubated at $4^{\circ} \mathrm{Co} / \mathrm{n}$ in a humidity chamber and thoroughly washed with PBS. The substrates were washed thoroughly with water and dried in a flow of dry $\mathrm{N}_{2}$ prior to SEM measurements.

Subsequently, one half of the substrate was incubated in a solution of goat $\mathrm{IgG}$ $\mathrm{Au}^{40}$ particles for 12 hours while the other half of the substrate was incubated in a solution of HRP-Au ${ }^{40}$ particles also for 12 hours. After incubation, all substrates were thoroughly washed with PBS and then MQ water, dried with nitrogen and imaged using a Phillips XL30 scanning electron microscope.

\subsubsection{DF images analysis}

An in-house Matlab program was used to analyze the images. The main output of this program is the colour of all (individual) nanoparticles present in the field of view in time. The colour of the nanoparticle, which is directly dependent on the spectrum of the nanoparticle, is given by the $\mathrm{r} / \mathrm{g}$ ratio, defined as the ratio between the intensities of the red and the green channel of the camera. The spectral calibration of the $\mathrm{r} / \mathrm{g}$ parameter was done with a fiber spectrometer. The spectra of multiple individual nanoparticles were measured while flowing glucose solutions of different concentrations and thus, different refractive indices, through the flowcell, in which the particles were immobilized. The $\mathrm{r} / \mathrm{g}$ to refractive index and wavelength calibration is illustrated in Figure 4-4. The wavelength calibration curve, illustrated in Figure $4 \mathrm{a}$ ) shows that the change of $\mathrm{r} / \mathrm{g}$ is proportional to 
4. Detection of individual binding events in a direct immunoassay

the spectral changes of the AuNP [28]. According to the calibration, in the wavelength range around $550 \mathrm{~nm}$ a change of $0.1 \mathrm{r} / \mathrm{g}$ corresponds to a peak shift of $5 \mathrm{~nm}$ [30].

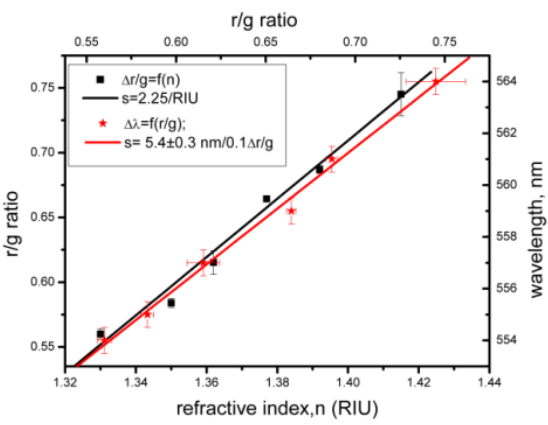

(a)

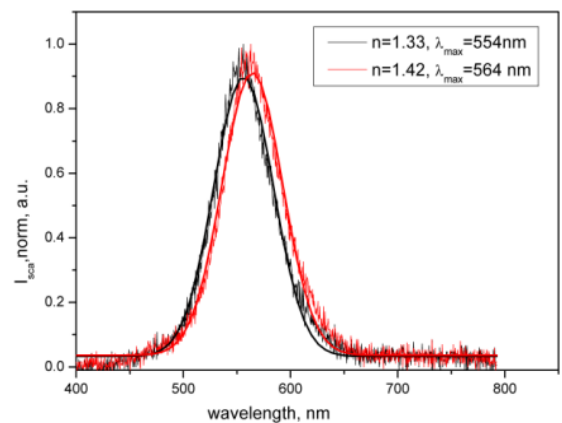

(b)

Figure 4-4 a) The wavelength and refractive index calibration of the colour camera on a individual $60 \mathrm{~nm}$ AuNp. b) The scattering spectra of the same particle in $n=1.33$ (black) and $n=1.42$ (red).

The procedure for the analysis consists of several steps. First, all particle positions are determined from a reference image. We defined the image preceding any change in the system as reference. Then a background subtraction is carried out for all images. A number of criteria are set (area, roundness and $\mathrm{r} / \mathrm{g}$ of the nanoparticles) to select the particles of interest and for the selected particles $\mathrm{r} / \mathrm{g}$ was monitored for each of the consecutive images. The output of the analysis program contains the position, intensities of the colour channels and the r/g of all selected particles in time. On the other hand, the "subtraction approach" provides a quick estimate of the total number of particles that had at least one other particle binding to it. For this procedure two images are needed: a reference image, taken at the beginning of the experiment and one image at the end of the experiment.

This procedure consists of the following steps. After all particles have been localized a background correction of the two images is performed. The same criteria as in the main program are used to select the particles of interest and for those particles the final $\mathrm{r} / \mathrm{g}$ is subtracted from the initial. Then limits are set $(0.1<\Delta \mathrm{r} / \mathrm{g}<0.5)$ and only those particles are counted that experienced a change of the $\mathrm{r} / \mathrm{g}$ within those limits. 


\section{Results and discussions}

\subsection{DF results}

Figure 4-5 shows the time traces of 4 different $\alpha$ goat- $\mathrm{Au}^{80}$ particles from the immunoassay (sample $\alpha$ goat- $\mathrm{Au}^{80}$ - goat $\mathrm{IgG}-\mathrm{Au}^{40}$ ) showing a change in colour (i.e. in $\mathrm{r} / \mathrm{g}$ ) during the goat $\mathrm{IgG}-\mathrm{Au}^{40}$ binding.

During the 30 minutes washing period, in the beginning of the experiment, the stability of the system was checked. The fluctuations of the system range between 0.01$0.02 \mathrm{r} / \mathrm{g}$, which is considered the noise level. The noise increases to 0.03-0.04 r/g during the goat IgG-Au $\mathrm{Au}^{40}$ incubation and decreases back to the starting level during washing. We suspect this increase during incubation is due to still freely diffusing particles in the solution during incubation that are temporarily approaching immobilized particles. The results show that for $41 \%$ of the analyzed particles $\left(\mathrm{N}_{\text {total }}=122\right)$ a stepwise increase of the $\mathrm{r} / \mathrm{g}$ occurs at different instances at times independent of each other. These discrete signal changes are much larger than the noise level during washing and remain stable for the entire rest of the experiment, even after washing. The discrete $\mathrm{r} / \mathrm{g}$ changes, with the calibration data in mind, correspond to at least $5 \mathrm{~nm}$ wavelength shifts, which are in good agreement with the calculations (Figure 1). Thus, we interpret the discrete increases of r/g as distinct binding events associated with a single (or few) particle(s), p2, to the immobilized particle, p1, in question.

As can be seen in Figure 4-5 different step sizes are observed. These differences were to be expected (see above) and can be the result of a number of factors like particle size, relative arrangement of the aggregate and interparticle distances also discussed thoroughly by Sannomiya et al. [25].

As (negative) control, we carried out the experiment in exactly the same way as before with the only change being the use of HRP-Au ${ }^{40}$ for incubation, which should not bind to $\alpha g o a t-\mathrm{Au}^{80}$. In Figure 4-6 traces of four individual particles are shown with a white background indicating washing with PBS and a yellow background indicating incubation with HRP-Au ${ }^{40}$. 


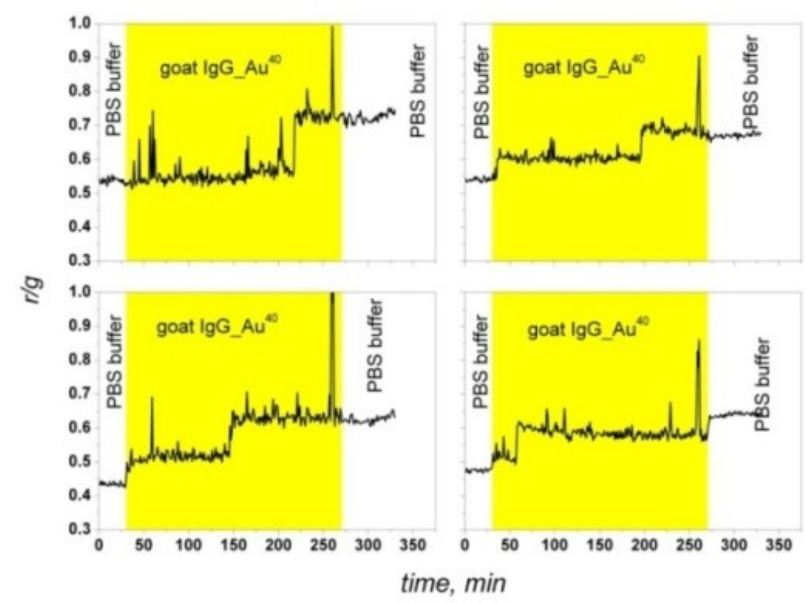

Figure 4-5 Time traces of $\mathrm{r} / \mathrm{g}$ of 4 individual particles from the immuno assay ( $\alpha$ goat- $\mathrm{Au}^{80}-$ goat $\left.\mathrm{Ig} G-\mathrm{Au}^{40}\right)$. The washing steps are indicated by a white background while the incubation period with goat $\mathrm{IgG}-\mathrm{Au}^{40}$ is indicated by a yellow background.

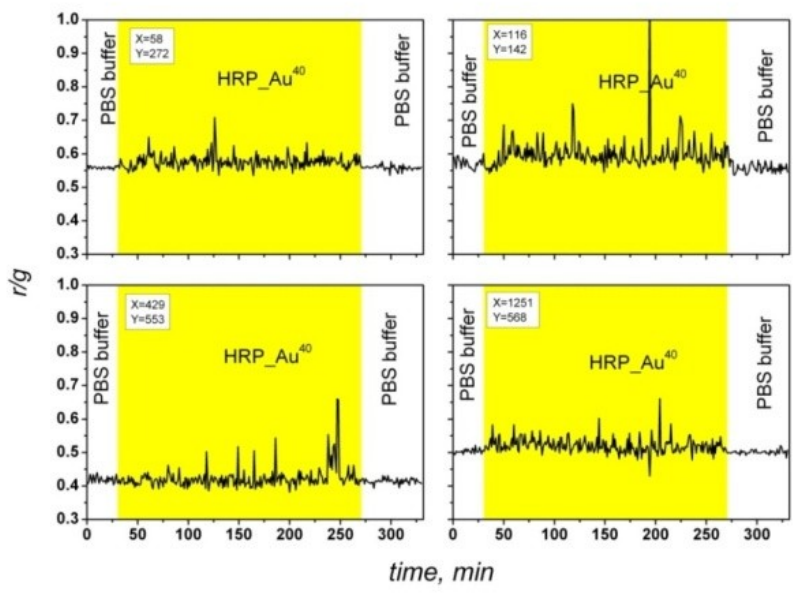

Figure 4-6 Time traces of $\mathrm{r} / \mathrm{g}$ of 4 individual particles from the reference ( $\alpha$ goat-Au $\mathrm{Au}^{80}-\mathrm{HRP}$ $\left.\mathrm{Au}^{40}\right)$. Washing steps are indicated by regions with white background, while incubation with HRP$\mathrm{Au}^{40}$ is indicated by a region with yellow background.

In general, a number of similarities were observed between the reference and sample signal traces: the noise level during washing was 0.01-0.02 r/g and increased to 
4. Detection of individual binding events in a direct immunoassay

0.03-0.05 r/g during the incubation of HRP-Au ${ }^{40}$. For 4\% of the analyzed particles $\left(\mathrm{N}^{\text {total }}\right.$ $=208$ ) a step-like increase was observed and only in half of those particles the signal was irreversible even after washing.

\subsection{SEM results}

Figure 4-7 shows typical SEM images of the sample (Figure 4-7a) and reference (Figure 4-7b). The $80 \mathrm{~nm}$ particles are clearly distinguishable from the $40 \mathrm{~nm}$ particles and bound particles can be easily recognized. In the case of the goat IgG-Au${ }^{40}$ (Figure 4-7a) more particles have been adsorbed non-specifically to the surface than in the case of HRP-Au ${ }^{40}$ (Figure 4-7b). This could possibly be due to a higher affinity of the goat IgG (as compared to that of HRP) toward the carboxylic acids groups covering the surface. The reference data show $980 \mathrm{~nm}-40 \mathrm{~nm}$ pairs out of 208 particles (in 15 images) while the sample shows 167 out of 259 (in 15 images) such pairs.

(a)
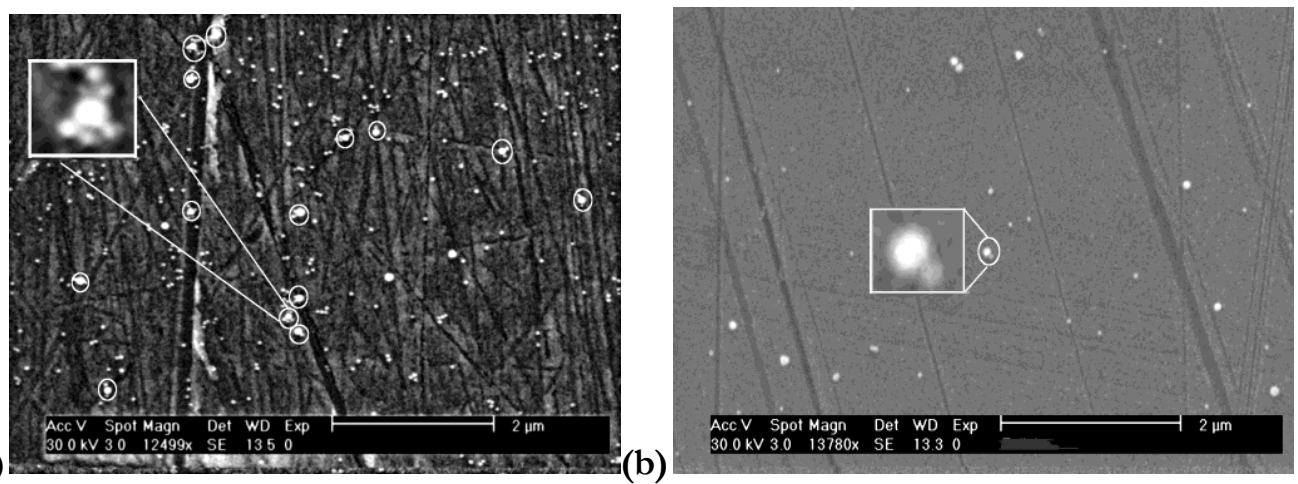

Figure 4-7 SEM images of the sample (a) and reference immunoassay (b). In both images, the 80 $\mathrm{nm}$ particles with at least one $40 \mathrm{~nm}$ particle binding to them are tagged with white circles. For two of those particles a zoom is shown as inset.

We determined the fraction of the $80 \mathrm{~nm}$ particles having $40 \mathrm{~nm}$ particles bound to them from the SEM images as 0.64 in the case of the sample and 0.04 in the case of the (negative) reference. 
These results were directly compared with the DF data where we determined the fraction of the $80 \mathrm{~nm}$ particles having at least one $40 \mathrm{~nm}$ particles bound to them using a subtraction program. Here, the last image of the DF experiment was subtracted from the initial image and only the particles that showed a change were counted, as shown in Figure 4-8.

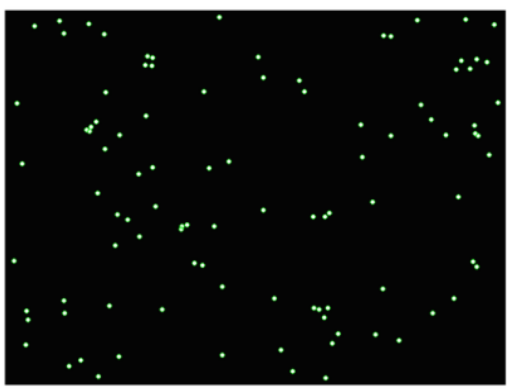

(a)

Figure 4-8 DF images of sample (left) and reference (right) after using the subtraction program, indicating only those particles that show a significant $\mathrm{r} / \mathrm{g}$ change.

To exclude false positives limits of $0.1<\Delta \mathrm{r} / \mathrm{g}<0.5$ were set. The lower limit was determined taking into account the minimum expected peak shift as obtained from the simulations and correlated with the calibration data. The higher limit was set to exclude the erroneous counting of particles covered by e.g. aggregates or dust particles, which we sometimes observed.

\begin{tabular}{c|cccc} 
& \multicolumn{2}{|c}{ DF } & \multicolumn{2}{c}{ SEM } \\
& Sample & reference & Sample & reference \\
\hline Bound & 50 & 4 & 167 & 9 \\
Unbound & 72 & 87 & 88 & 199 \\
Total & 122 & 91 & 259 & 208
\end{tabular}

Table 4-1 Comparison between DF and SEM data presenting the number of bound and unbound particles.

The results are shown in Table 4-1. Binding fractions obtained from SEM (0.64 for sample, respectively 0.04 for reference) data are in reasonable agreement with those 
4. Detection of individual binding events in a direct immunoassay

obtained from darkfield experimental data ( 0.41 for sample, respectively 0.04 for reference). The differences between the two sets of data could be due to the difference in incubation time: for the SEM experiments incubation was done for 12 hours for the DF experiment for only 4 hours.

\subsection{Experimental determination of the surface coverage, $\Gamma$}

From the DF experiments the immuno-binding may be analyzed in time. Integrating over all binding events of all analyzed particles as a function of time results in Figure 9, which represents a typical binding curve, as is routinely found in ensemble assays.

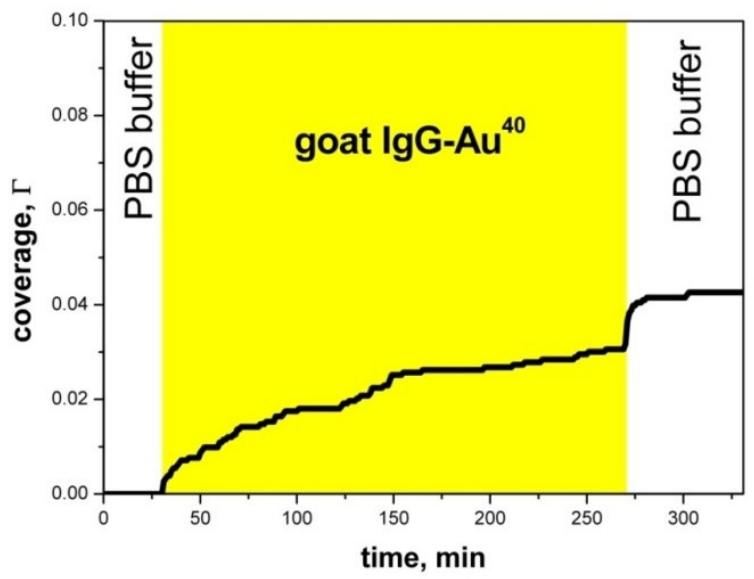

Figure 4-9 The time-dependent immuno-binding of goat IgG-Au ${ }^{40}$ particles to $\alpha$ goat- $\mathrm{Au}^{80}$ particles in the DF. Integration of each binding event of all analyzed particles in time.

In order to establish the viability of these results we compared the theoretically expected fraction of occupied binding sites on the surface, $\Gamma^{\text {theory }}$, with the experimentally determined $\Gamma$ experiment.

In the following we assumed the Langmuir binding isotherm to be valid. Then the following relation holds: 


$$
\Gamma=\frac{K_{A[A]}}{1+K_{A[A]}}
$$

where $\Gamma$ is the fraction of surface immobilized receptor molecules with a target molecule bound to them, $[\mathrm{A}]$ is the concentration of target molecules in solution and $\mathrm{K}_{\mathrm{A}}$ is the affinity constant of the surface reaction under consideration. For the immunoreaction considered here we have found that $\mathrm{K}_{\mathrm{A}} \approx 10^{8} \mathrm{M}^{-1}$, as determined using SPR (data not shown).

We estimated that a maximum of $18 \mathrm{Au}^{40}$ fit on the surface of an immobilized $\mathrm{Au}^{80}$ and that at close approach of an $\mathrm{Au}^{40}$ to an $\mathrm{Au}^{80}$ conjugate a number, $\mathrm{m}$, of about 10 antibodies attached to the $\mathrm{Au}^{40}$ could bind simultaneously to the $\mathrm{Au}^{80}$. This situation implies that multivalent binding occurs, where, due to a high local density of binding sites the affinity constant, $K_{A}$, increases significantly [35].For the situation of first approach of an $\mathrm{Au}^{40}$ conjugate to the surface covered with binding sites the affinity constant will be multiplied by a factor $\mathrm{m}$ resulting in a modified expression (4.2) for the fractional coverage.

$$
\Gamma=\frac{m K_{A[A]}}{1+m K_{A[A]}}
$$

The fractional coverage determined using expression 4.2 represents the lowest bound and will probably be exceeded in reality as desorption is expected to become very slow due to the highly multivalent nature of the binding. Thus, using equation4.2, considering a concentration of $\mathrm{Au}^{40}$ of $0.1 \mathrm{nM}$ (obtained from extinction measurements, results not shown) yields an estimate for the expected coverage, $\Gamma^{\text {theory }}$ of 0.09 .

The determination of $\Gamma$ experiment (again taking into account that the estimate of the upper limit of the number $\mathrm{Au}^{40}$ fitting on the accessible surface of an immobilized $\mathrm{Au}^{80}$ is 18) yields a $\Gamma^{\text {experiment }}=$ number of single nanoparticle binding events / (number immobilized particles $*$ number $\mathrm{Au}^{40}$ fitting on a $\left.\mathrm{Au}^{80}\right)=$ number of single nanoparticle binding events / total number available binding sites $=57 /(122 * 18)=0.03$, which is in reasonable agreement with the expected fractional coverage, $\Gamma^{\text {theory }}$ of 0.09 . 


\section{Conclusions}

In conclusion, we show here for the first time the parallel detection of hundreds of individual binding events of goat IgG-Au${ }^{40}$ particles to $\alpha$ goat- $\mathrm{Au}^{80}$ particles using a color camera. Binding events associated with a single nanoparticle were observed as steplike changes of colour expressed as an increase of an $\mathrm{r} / \mathrm{g}$ parameter. The step size, correlated with the calibration data, show good agreement with our DDSCAT calculation when considering binding events associated with a single nanoparticle. To corroborate these data SEM experiments have been carried out and are in good agreement with the DF data. Together with the good agreement of the experimentally confirmed fractional surface coverage with the theoretically expected value this confirms the validity of our findings. We believe that an extension of our approach, where the target protein molecule is sandwiched between two properly conjugated Au nanoparticles can lead to a novel immunosensing platform with single protein molecule detection capabilities. The sensing technique we have presented here combines single molecule sensitivity with parallel detection ability, multiplexing and general applicability to most specific binding reactions. Our approach has the possibility to become a standard detection method with superior sensitivity.

Acknowledgements: We would like to thank Prof. Dr. Mark McDermott, University of Alberta, Edmonton, Canada and NINT, Edmonton, Canada for his kind invitation to D.W. to work at his labs as well as his generous hospitality and the opportunity to utilize all the facilities there; Ing. Robert Molenaar University of Twente, Enschede, Netherlands for the technical support with the DF setup and Prof. Dr. Jurriaan Huskens, University of Twente, Enschede, Netherlands for enlightening discussions.

This work was financially supported by MicroNed (project nr. WP2F-FoAc) and the European Union (FP6 STREP Project Fluoromag (Proj. nr. 037465)). 


\section{References}

[1] V. Kanda, J.K. Kariuki, D.J. Harrison, M. T. McDermott, Label-Free Reading of MicroarrayBased Immunoassays with Surface Plasmon Resonance Imaging, Analytical Chemistry 76 (2004) 7257.

[2] C. Björkman, O. J. M. Holmdahl, A. Uggla, An indirect ensyme-linked immunoassay (ELISA) for demonstration of antibodies to Neospora caninum in serum and milk of cattle, Veterinary Parasitology 68 (1997) 251.

[3] C. Sönnichsen, S. Geier, N. E. Hecker, G. von Plessen, J. Feldmann, H. Ditlbacher, B. Lamprecht, J. R. Krenn, F. R. Aussenegg, V. Z. H. Chan, J. P. Spatz, M. Moller, Spectroscopy of single metallic nanoparticles using total internal reflection microscopy, Applied Physics Letters 77 (2000) , 2949.

[4] J. J. Storhoff, A. D. Lucas, V. Garimella, Y. P. Bao, U. R. Muller, Homogeneous detection of unamplified genomic DNA sequences based on colorimetric scatter of gold nanoparticle probes, Nature Biotechnology 22 (2004) 883.

[5] R. A. Reynolds, C. A. Mirkin, R. L. Letsinger, Homogeneous, Nanoparticle-Based Quantitative Colorimetric Detection of Oligonucleotides, Journal of the American Chemical Society 122 (2000) 3795.

[6] K. A. Willets, R. P. Van Duyne, Localized Surface Plasmon Resonance Spectroscopy and Sensing, Annual Review of Physical Chemistry 58 (2007) 267.

[7] N. Nath, A. Chilkoti, A Colorimetric Gold Nanoparticle Sensor To Interrogate Biomolecular Interactions in Real Time on a Surface, Analytical Chemistry 74, (2001), 504.

[8] P. K. Jain, K. S. Lee, I. H. El-Sayed, M. A. El-Sayed, Calculated Absorption and Scattering Properties of Gold Nanoparticles of Different Size, Shape, and Composition: Applications in Biological Imaging and Biomedicine, The Journal of Physical Chemistry B 110 (2006), 7238.

[9] K. L. Kelly, E. Coronado, L. L. Zhao, G. C. Schatz, The Optical Properties of Metal Nanoparticles: The Influence of Size, Shape, and Dielectric Environment, The Journal of Physical Chemistry B 107 (2002) 668.

[10] G. Raschke, S. Kowarik, T. Franzl, C. Sonnichsen, T. A. Klar, J. Feldmann, A. Nichtl, K. Kurzinger, Biomolecular Recognition Based on Single Gold Nanoparticle Light Scattering, NanoLetters 3 (2003) 935. 
4. Detection of individual binding events in a direct immunoassay

[11] K. E. Nelson, L. Gamble, L. S. Jung, M. S. Boeckl, E. Naeemi, S. L. Golledge, T. Sasaki, D. G. Castner, C. T. Campbell, P. S. Stayton, Surface Characterization of Mixed Self-Assembled Monolayers Designed for Streptavidin Immobilization, Langmuir 17 (2001) 2807.

[12] J. J.Storhoff, A. A. Lazarides, R. C. Mucic, C. A. Mirkin, R. L. Letsinger, G. C. Schatz, What Controls the Optical Properties of DNA-Linked Gold Nanoparticle Assemblies?, Journal of the American Chemical Society 122 (2000) 4640.

[13] R. Elghanian, J. J. Storhoff, R. C. Mucic, R. L. Letsinger, C. A. Mirkin, Selective Colorimetric Detection of Polynucleotides Based on the Distance-Dependent Optical Properties of Gold Nanoparticles,Science 277 (1997) 1078-1081.

[14] L. M. Demers, C.A. Mirkin, R. C. Mucic, R. A. Reynolds, R. L. Letsinger, R. Elghanian, G. Viswanadham,A Fluorescence-Based Method for Determining the Surface Coverage and Hybridization Efficiency of Thiol-Capped Oligonucleotides Bound to Gold Thin Films and Nanoparticles,Analytical Chemistry $72(2000) 5535$.

[15] A. D. McFarland, R. P. Van Duyne, Single Silver Nanoparticles as Real-Time Optical Sensors with Zeptomole Sensitivity, NanoLetters 3 (2003) 1057.

[16] S. M. Marinakos, S. Chen, A. Chilkoti, Plasmonic Detection of a Model Analyte in Serum by a Gold Nanorod Sensor, Analytical Chemistry 79 (2007) 5278.

[17] K. M. Mayer, S. Lee, H. Liao, B. C. Rostro, A. Fuentes, P. T. Scully, C. L. Nehl, J. H. Hafner, A Label-Free Immunoassay Based Upon Localized Surface Plasmon Resonance of Gold Nanorods, ACS Nano 2 (2008) 687.

[18] G. J. Nusz, S. M. Marinakos, A. C. Curry, A. Dahlin, F. Hook, A. Wax, A. Chilkoti, LabelFree Plasmonic Detection of Biomolecular Binding by a Single Gold Nanorod, Analytical Chemistry 80 (2008) 984.

[19] S. Link, M. A. El-Sayed, Size and Temperature Dependence of the Plasmon Absorption of Colloidal Gold Nanoparticles, The Journal of Physical Chemistry B 103 (1999) 4212.

[20] M. D. Malinsky, K.L. Kelly, G. C. Schatz, R.P. Van Duyne, Chain Length Dependence and Sensing Capabilities of the Localized Surface Plasmon Resonance of Silver Nanoparticles Chemically Modified with Alkanethiol Self-Assembled Monolayers, Journal of the American Chemical Society 123 (2001) 1471. 
4. Detection of individual binding events in a direct immunoassay

[21] W. Rechberger, A. Hohenau, A. Leitner, J. R. Krenn, B. Lamprecht, F. R. Aussenegg, Optical properties of two interacting gold nanoparticles, Optics Communications 220 (2003) 137.

[22] J. B. Lassiter, J. Aizpurua, L. I. Hernandez, D. W. Brandl, I. Romero, S. Lal, J. H. Hafner, P. Nordlander, N. J. Halas, Close Encounters between Two Nanoshells, NanoLetters 8 (2008) 1212.

[23] P. Olk, J. Renger, M. T.Wenzel, L. M. Eng,Distance Dependent Spectral Tuning of Two Coupled Metal Nanoparticles, NanoLetters 8 (2008) 1174.

[24] J. Zhao, A. Das, G. C. Schatz, S. G. Sligar, R. P.Van Duyne, Resonance Localized Surface Plasmon Spectroscopy: Sensing Substrate and Inbibitor Binding to Cytochrome P450,The Journal of Physical Chemistry C 112 (2008) 13084.

[25] T. Sannomiya, C. Hafner, J. Voros, In situ Sensing of Single Binding Events by Localized Surface Plasmon Resonance, NanoLetters 8 (2008) 3450.

[26] A. Haes, D. Stuart, S. Nie, R. Van Duyne, Using Solution-Phase Nanoparticles, Surface-Confined Nanoparticle Arrays and Single Nanoparticles as Biological Sensing Platforms, Journal of Fluorescence 14 (2004) 355.

[27] J. J. Mock, D. R. Smith, S. Schultz, Local Refractive Index Dependence of Plasmon Resonance Spectra from Individual Nanoparticles, NanoLetters 3 (2003) 485.

[28] C. L. Baciu, J. Becker, A. Janshoff, C. Sönnichsen, Protein Membrane Interaction Probed by Single Plasmonic Nanoparticles, NanoLetters 8 (2008) 1724.

[29] R. Verdoold, F. Ungureanu, D. Wasserberg, R. P. H. Kooyman, Gold nanoparticle assays: towards single molecule unamplified DNA detection, Advanced Environmental, Chemical, and Biological Sensing Technologies VI 7312 (2009) 73120N.

[30] F. Ungureanu, J. Halamek, R. Verdoold, R. P. H. Kooyman, The use of a colour camera for quantitative detection of protein-binding nanoparticles, Plasmonics in Biology and Medicine VI 7192 (2009) 719200 .

[31] B. T. Draine, P. J. Flatau, Discrete-dipole approximation for scattering calculations, Journal of the Optical Society of America A 11 (1994), 1491.

[32] P. B. Johnson, R. W. Christy, Optical Constants of the Noble Metals, Physical Review B 6 (1972) 4370. 
4. Detection of individual binding events in a direct immunoassay

[33] S. W. Prescott, P. Mulvaney, Gold nanorod extinction spectra, Journal of Applied Physics 99 (2006) 123504.

[34] C. Ungureanu, R. G. Rayavarapu, S. Manohar, T. G. van Leeuwen, Discrete dipole approximation simulations of gold nanorod optical properties: Choice of input parameters and comparison with experiment, Journal of Applied Physics 105 (2009) 102032.

[35] J. Huskens, A. Mulder,, T. Auletta, C. A. Nijhuis,, M. J. W. Ludden, D. N. Reinhoudt, A Model for Describing the Thermodynamics of Multivalent Host-Guest Interactions at Interfaces, Journal of the American Chemical Society126 (2004), 6784-6797

[36] A. R. de Boer, C. H. Hokke, A.M. Deelder, M. Wuhrer, Serum Antibody Screening by Surface Plasmon Resonance Using a Natural Glycan Microarray, Glycoconjucate Journal 25 (2008), 75-84

[37] J. B. Beusink , A. M.C. Lokate, G. A.J. Besselink ,G. J.M. Pruijn, R. B.M. Schasfoort, AngleScanning SPR Imaging for Detection of Biomolecular Interactions on Microarrays, Biosensors and Bioelectronics 23 (2008) 839-844

[38] A. Gunnarson, P. Jonnson, R. Marie, J. O. Tegenfeld, F. Hook, Single Molecule Detection and Mismatch Discrimination of Unlabelled DNA Targets, NanoLetters 8 (2008), pp.183-188

[39] Y. Teramura, Y. Arima, H. Iwata, Surface Plasmon resonance-based bighly sensitive immunosensing for brain natriuretic peptide using nanobeads for signal amplification, Analytical biochemistry 2006, 357, 208-15 


\section{Chapter 5}

\section{Performance of colorimetric darkfield microscopy in an amplified sandwich immunoassay}

Abstract: In previous chapters, we have established that colorimetric DF microscopy can be employed for the detection and quantification of biomolecular interactions in a label free format and have determined the LOD of the method. In addition, we demonstrated that when an amplification sensing strategy is used, single binding events could be observed. This was established in an amplified direct protein immunoassay. In this chapter, we describe a preliminary investigation of the detection of individual binding events in an amplified sandwich immunoassay. Finally, the LOD of our sensing approach in the amplified sandwich immunoassay is examined. 


\section{Introduction}

Present golden standards in sensitive detection are the ELISA test for proteins and the PCR approach for DNA detection. Both methods require expensive reagents, are time consuming and require trained personnel. Therefore, there is extensive research on new, non-complicated and inexpensive detection strategies [1,2]. An alternative in the sensitive detection of biomolecules is offered by the surface plasmon resonance (SPR) technique that is based on the monitoring of changes in the refractive index of the substrate due to molecular binding [3-6]. It offers a reasonably high sensitivity, label-free detection and extensive multiplexing capabilities. However, the detection of low molecular weight molecules is still challenging.

Another approach involves the use of various optical reporters [7,8]. Fluorescent labels are widely employed to detect the presence of target molecules, as they are available on a large scale and in sufficient variety [9]. However, they suffer from several drawbacks: (1) photobleaching, (2) low signal of a single reporter requiring more reporters at the same site for a reasonable detection and (3) quenching of the fluorescence when the fluorophores are too closely packed together. In addition, a complex dedicated system is necessary for sensitive detection. An alternative is to use gold or silver nanoparticles as tags, which do not have the inherent disadvantages of fluorophores [10]. The scattering intensity of one $60 \mathrm{~nm}$ Au nanoparticle is equivalent to $10^{5}$ fluorophores and is under DF conditions easily observable with the naked eye [11]. This is due to the optical phenomenon LSPR, explained in chapter 2 of this thesis. Colorimetric methods based on the aggregation of the nanoparticles due to specific binding are widely used [12]. Although it is a fast and reliable method and an LOD is obtained in the $\mathrm{nM}$ range, single binding events cannot be observed. An alternative is the use of individual nanoparticles as sensing platforms [13-15].

The detection in this type of biosensors is based on measuring changes, due to binding events, in the extinction spectra of individual Au nanoparticles [16-18] using a spectrophotometer. The Van Duyne group has demonstrated the potential of individual nanoparticles as sensing platforms by detecting the binding of a small number of 
5. Performance of colorimetric darkfield microscopy in an amplified sandwich immunoassay molecules on the surface of a silver nanoparticle [16]. Although impressive, in many sensing applications of utmost interest is the concentration of target molecules that can be detected. This can be achieved by massive parallelization of individual nanoparticles. When measuring the changes in the spectra of individual particles using a spectrometer, parallelization is difficult to obtain. Baciu et al. and more recently Chen et al.[19,20] succeeded to measure simultaneously the spectra of a limited number of particles by using LCTF filters; however this is not enough to measure very low concentrations. In addition, this method is time consuming, and expensive.

In chapters 3 and 4, we introduced the concept of using a colour camera to detect single binding events on multiple individual nanoparticles simultaneously [21,22]. Here we demonstrated the parallel capabilities of a colour camera in a protein assay. With this system, we were able to detect changes of the spectral properties of individual particles due to binding events by monitoring the changes of the colour of those particles. Additionally, we were able to monitor the kinetics of the binding processes of the biomolecule of interest. In the unamplified immunoassay, the binding of the target to the receptor molecules attached on the immobilized nanoparticles were detected as continuous changes in the signal while in amplified direct immunoassays each binding event was perceived as a step-like increase in the signal.

In this chapter, we use our sensing strategy for the detection of target molecules in a protein sandwich immunoassay (cf. fig. 5-1) using $80 \mathrm{~nm}$ gold particles immobilized on the surface, conjugated with receptor molecules, and $40 \mathrm{~nm}$ functionalized gold particles as labels. Additionally, we experimentally determined the sensitivity of our sensing strategy, in terms of concentration, in this type of application. 


\section{Materials and methods}
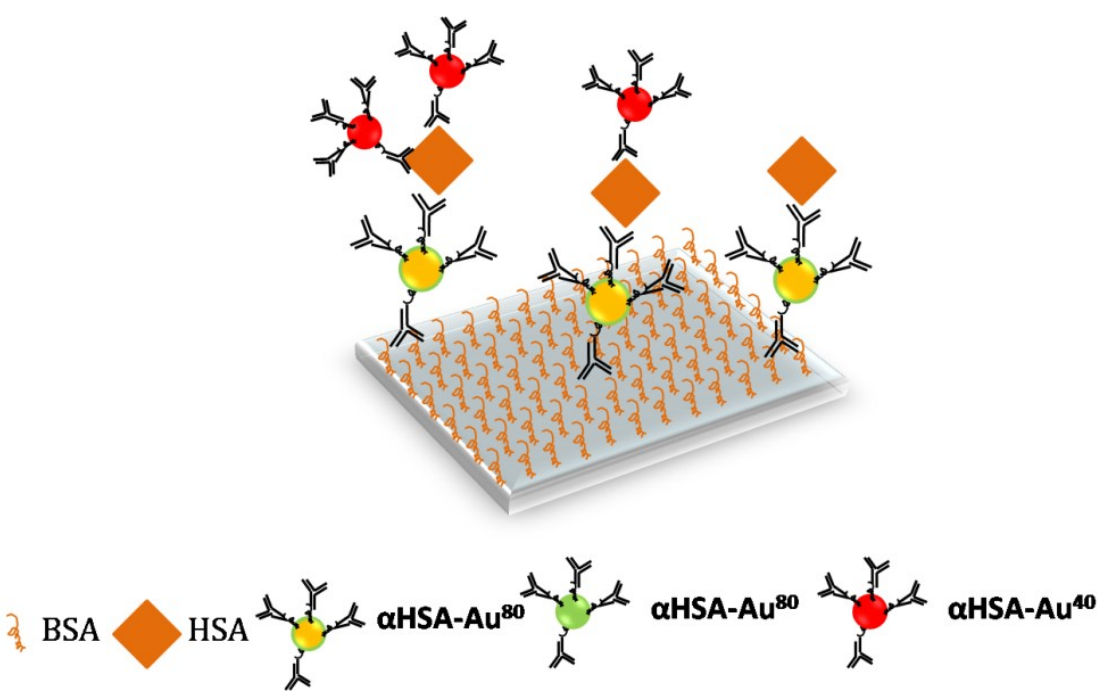

Figure 5-1 Artistic representation of the sandwich assay performed in this study: The 'developer' $\left(\alpha \mathrm{HSA}-\mathrm{Au}^{40}\right)$ will bind to the target molecules (HSA); this is bound to the receptor molecules $(\alpha \mathrm{HSA})$ found on the immobilized nanoparticle.

Here, we investigated the detection of individual binding events in an amplified sandwich immunoassay. This was done by performing two sets of assays:

(1) sample: The binding of the developer $\left(\alpha \mathrm{HSA}-\mathrm{Au}^{40}\right)$ to the target molecules (HSA)

and

(2) control: The lack of binding of the developer $\left(\alpha H S A-A u^{40}\right)$ when HRP molecules are used as target molecules.

For the experimental determination of the LOD, we used different concentrations of target molecules, labelled them, and subsequently evaluated the covered fraction for each concentration. 
5. Performance of colorimetric darkfield microscopy in an amplified sandwich immunoassay

\subsection{Assay design: choice of the nanoparticle pair}

We carried out a series of calculations to estimate the spectral shifts when two nanoparticles are in close proximity. These studies were conducted using the DDSCAT software, developed by Draine and Flatau [23]. This was successfully used in other studies of the optical properties of metallic nanoparticles [24, 25]. In short, this program uses the discrete dipole approximation method to iteratively calculate the optical properties of nanoparticles. The investigated particle is divided into $\mathrm{N}$ interacting dipoles and the electromagnetic field of the particle is calculated from their interaction with the incident electromagnetic field. In simulations the bulk dielectric function of gold was used [26] and water with a refractive index of 1.33 was considered as surrounding medium. Results of these simulations are illustrated in chapter 2, Section 5.2, Figure 2-11a.

The close proximity of two nanoparticles in a sandwich, due to the binding of the target molecules to the receptor molecules placed on the nanoparticles, leads to a shift in the resonance conditions resulting in an observable spectral shift. As illustrated in Figure 2-11a the size of this effect is influenced by the inter-particle distance and the dimensions of the involved particles [27-30]. Hence, in order to have large spectral shifts we need to have lower inter-particle distances and larger particles. However, the inter-particle distance is dictated by the size of the target and receptor molecules involved and cannot be changed. Therefore, the parameter that can be changed is the size of the particles involved in the assay. In order to minimize the LOD a large number of receptors on the particle's surface is preferred. This can be achieved if larger particles are used [31, 32]. However, the stability of larger conjugated particles is relatively low [33].

Additionally, the visualization of larger immobilized individual nanoparticles under DF microscopy is easier since the scattering cross-section of these particles is larger. The size of the developer (cf. fig. 5-1) is chosen such that it gives a large PP shift upon binding, and at the same time a low additional background signal during the incubation period (that is, the scattering cross - section of the developer must be low). In addition, in characterization methods like scanning electron microscopy (SEM) the occurrence of a 
5. Performance of colorimetric darkfield microscopy in an amplified sandwich immunoassay binding event due to specific interaction is easier recognizable when the assay involves the use of two particles of different sizes.

Hence, based on these selection criteria and the results of our simulations we developed an assay made from a large immobilized nanoparticle $\left(\mathrm{Au}^{80}\right)$ and a smaller one $\left(\mathrm{Au}^{40}\right)$ as 'developer'. The $\mathrm{Au}^{40}$ are chosen such that upon binding a large PP shift is provided and a lower background signal during the developer step is added.

For the immunoassay as considered here $\left(\alpha \mathrm{HSA}-\mathrm{Au}^{80}-\mathrm{HSA}-\alpha \mathrm{HSA}-\mathrm{Au}^{40}\right)$, the inter-particle distance (surface-to-surface), was estimated to be $25 \mathrm{~nm}$. According to our calculation results, this distance corresponds to a $\Delta \lambda_{\max } \sim 7 \mathrm{~nm}$. On the other hand, the magnitude of the expected wavelength shift is dependent on the direction of the axis of the particle pair relative to the polarization direction of the light as discussed in chapter 2 , section 5.2. Under these conditions, we expect at a single binding event in the sandwich immunoassay a wavelength shift varying from $\sim 3$ to $15 \mathrm{~nm}$.

\subsection{Bioconjugation of gold nanoparticles}

All chemicals and proteins were used without any further purification. A scheme of the bioconjugation protocol is depicted in Figure 5-2. All buffers were prepared using milliQ (MQ) water.

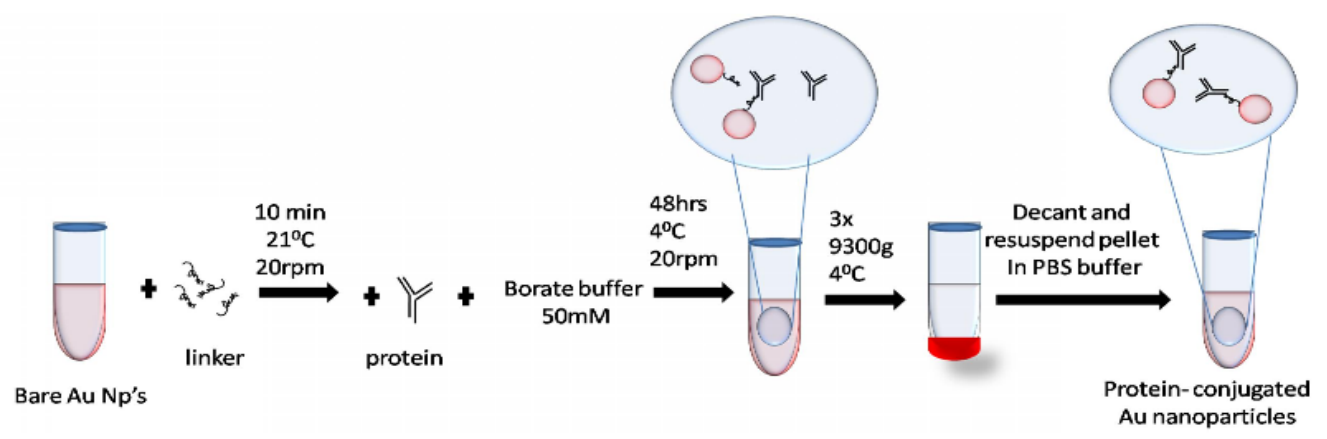

Figure 5-2 Schematic representation of the conjugation protocol of $\mathrm{Au}$ nanoparticles with protein molecules 
5. Performance of colorimetric darkfield microscopy in an amplified sandwich immunoassay

Bare gold nanoparticles, $\mathrm{Au}^{80}$ and $\mathrm{Au}^{40}\left(2^{*} 10^{-14}\right.$ and $2^{*} 10^{-13} \mathrm{M}$, respectively, $\left.\mathrm{BBI}\right)$, were mixed for 10 minutes $\left(20 \mathrm{rpm}, 21^{\circ} \mathrm{C}\right)$ with the protein linker 2,5-dioxopyrrolidin-1-yl 26-mercapto-3,6,9,12,15-pentaoxahexacosyl carbonate (Prochimia, TH013). The fresh solution was added to $5 \mu \mathrm{M}$ solution of protein (HSA (Sigma Aldrich, A4327), $\alpha$ HSA (Sigma Aldrich, A9731) or HRP (VI, Sigma Aldrich, P6782)) in $50 \mathrm{mM}$ borate buffer. The final molar ratios were 1:50, 1:500 and 1:5000 Au NP:protein for all the combinations. The solutions were left to incubate in a rotator for $48 \mathrm{hrs}$ in a cool environment $\left(4^{\circ} \mathrm{C}\right.$ at $20 \mathrm{rpm})$. The excess of the protein was removed by repeated centrifugation $(3 \mathrm{x}, 40 \mathrm{~nm}$ particles: 9300g, RT and $80 \mathrm{~nm}$ particles $2300 \mathrm{~g}$, RT) of the solutions. The supernatant was removed and the pellets were resuspended in $5 \mathrm{mM}$ borate buffer $(\mathrm{pH}$ ) $)$ in the first 2 steps and in phosphate buffered saline (PBS) in the last step. The conjugate solutions were stored at $4^{\circ} \mathrm{C}$ in PBS buffer.

The absorbance spectra of the conjugated nanoparticles were measured using a spectrophotometer. The presence of the proteins on the nanoparticles was verified by measuring changes in the position of the peak in the absorbance spectra of the conjugate solution (data not shown).

\subsection{Sensor preparation}

For the preparation of the sensor, standard microscope glass slides BK7 $(76 \mathrm{~mm}$ x $26 \mathrm{~mm}$ x $1 \mathrm{~mm}$, Menzel, Germany) were used as substrates. First, the slides were activated by immersing them for $15 \mathrm{~min}$ in a freshly prepared piranha solution $3: 1$ mixture of $\mathrm{H}_{2} \mathrm{SO}_{4}: \mathrm{H}_{2} \mathrm{O}_{2}$ ) at $95^{\circ} \mathrm{C}$. Subsequently, the slides were sonicated for 15 minutes in MQ water and used immediately for further surface modification. A 5\% solution of 3amino propyl triethoxy silane (APTES) in EtOH (p.a., Merck) was prepared and the slides were incubated for 15 minutes. The excess of APTES was removed by repeated rinsing with MQ water and sonicated for 5 minutes using fresh MQ water each time. Then, the silanisation of the slides was finalized by a $2 \mathrm{~h}$ baking step at $120^{\circ} \mathrm{C}$. The slides, which were not used immediately, could be stored for a week at $120^{\circ} \mathrm{C}$ or immersed in EtOH at $4^{\circ} \mathrm{C}$. 
5. Performance of colorimetric darkfield microscopy in an amplified sandwich immunoassay

Subsequently, the silanized surfaces were passivated in order to suppress nonspecific binding (of the target and developer) on the substrate. Substrates were then incubated at $4^{\circ} \mathrm{C}$ in a humidity chamber using a $2 \mathrm{mM}$ solution of mercapto undecanoic acid (MUA, 450561 Sigma Aldrich) in a 1:1 mixture of EtOH and water. After incubation the substrates were thoroughly washed with EtOH and water and dried in a flow of dry $\mathrm{N}_{2}$. Then, the slides were incubated for 15 minutes in a solution of $0.3 \mathrm{M}$ of $\mathrm{N}$ hydroxysuccinimide (NHS, 56480 Fluka) mixed with a $0.04 \mathrm{M}$ solution of $\mathrm{N}$-(3Dimethylaminopropyl)-N'-ethylcarbodiimide (EDC, 03449 Aldrich). Then they were briefly washed with water and PBS and immediately incubated with the passivation agent for $2 \mathrm{hrs}$ at $4^{\circ} \mathrm{C}$ in a humidity chamber. As a passivating agent we used bovine serum albumine (BSA), commonly used in literature [34]. The passivated surface was then thoroughly washed with PBS and a second activation step, with $0.3 \mathrm{M}$ of NHS $+0.04 \mathrm{M}$ EDC solution, was initiated. After 15 minutes of incubation of the slides in this mixture and washing with water and PBS, the activated amino groups of the BSA coated slides were exposed to the $\alpha \mathrm{HSA}-\mathrm{Au}^{80}$ conjugates $\left(10^{-13} \mathrm{M}\right)$ for 10 minutes. After immobilization slides were rinsed thoroughly with PBS.

\subsection{Optical detection of the immunoassay}

The resulting AuNP-conjugate functionalized flowcell was mounted on our DF setup, as explained in chapter 3, Section 2.2. Shortly, the visualization of the nanoparticles was achieved using an Olympus GX71 inverted microscope in reflection mode, with an MPLAN $20 \mathrm{x}$ dark field objective, in combination with a 75 Watt Xe lamp as a light source. The images were acquired using a colour camera (Carl Zeiss HRc, 14 bit). In order to reduce objective focus fluctuations, a continuous auto-focus feedback loop was used. 


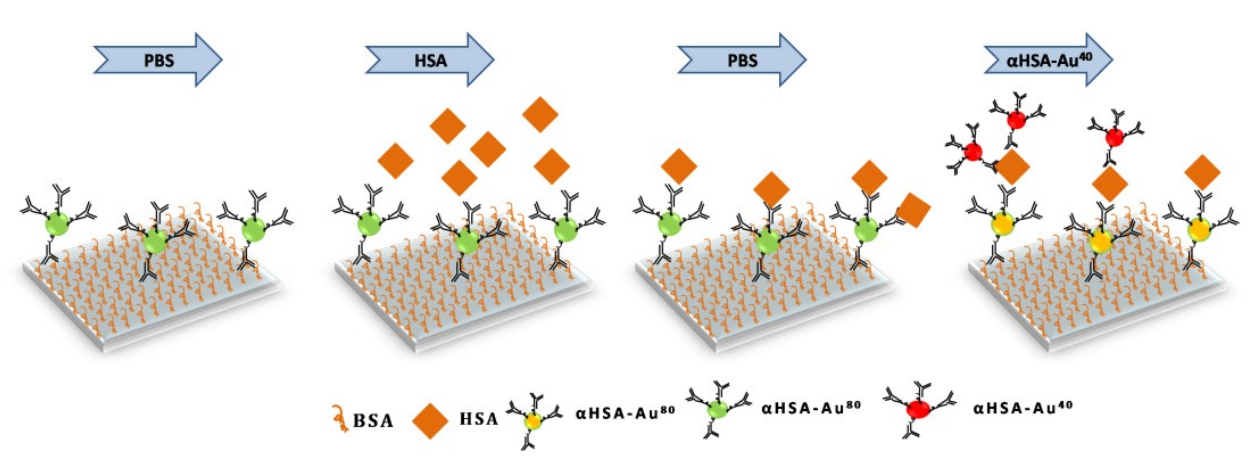

Figure 5-3 The flow assay-schematic representation. For details, see text.

The flow assays were conducted as follows (Figure 5-3):

1. 30 minutes washing with phosphate buffered saline $(\mathrm{PBS})($ flow rate $=14 \mu \mathrm{l} / \mathrm{min})$

2. Incubation with a solution containing a certain concentration of target molecules (HSA) for the sample and HRP $(300 \mathrm{nM})$ for the control, respectively) for 2 hours (no flow)

3. 10 minutes washing with PBS (flow rate $=14 \mu \mathrm{l} / \mathrm{min}$ )

4. Incubation with a solution of the developer the $\alpha \mathrm{HSA}_{-} \mathrm{Au}^{40}\left(10^{-13} \mathrm{M}\right)$ for $3 \mathrm{hrs}$ (no flow)

5. 20 minutes washing with PBS

The image recording was done as follows:

For the flow assay, the colour camera was utilised to record consecutive images (1 image/min) of all the individual nanoparticles present in the field of view. The acquisition time per image was set to 2.5 seconds. The images were captured using an in-house Labview acquisition program, which allows for the automatic recording of multiple consecutive images and aligns the consecutive images of one series compensating for possible lateral shifts during the course of a flow assay. 

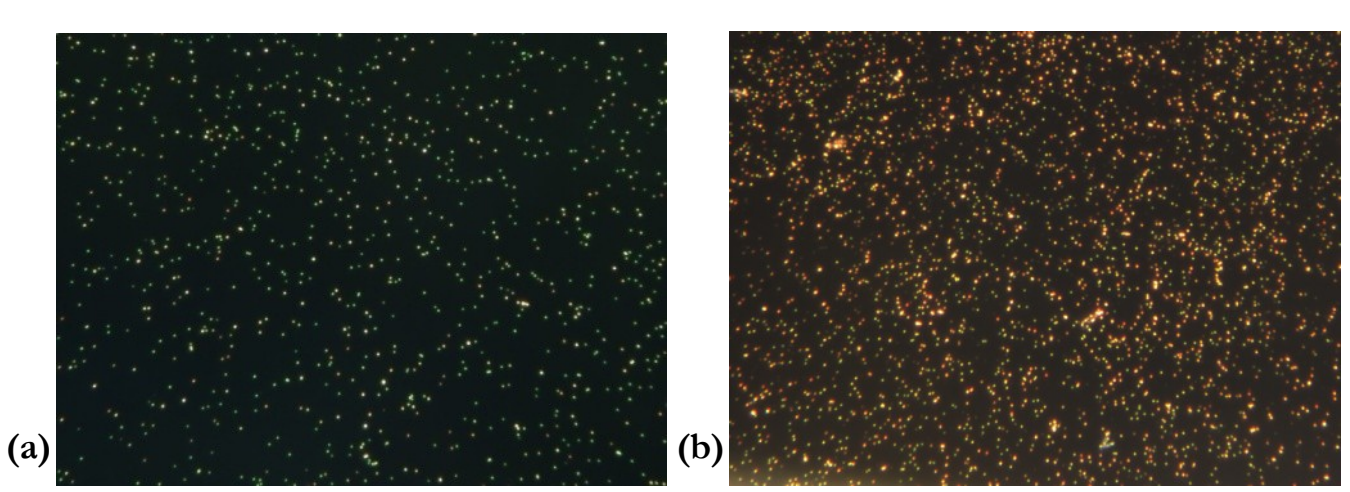

Figure 5-4 DF image of $\alpha \mathrm{HSA}-\mathrm{Au}^{80}$ immobilized on glass substrate (a) after the target molecules were incubated $\left(\alpha \mathrm{HSA}-\mathrm{Au}^{80}-\mathrm{HSA}\right)$ and $(\mathrm{b})$ at the end of the immuno - assay experiment ( $\alpha \mathrm{HSA}$ $\left.\mathrm{Au}^{80}-\mathrm{HSA}-\alpha \mathrm{HSA}-\mathrm{Au}^{40}\right)$. The FOV of the image is $270 \mathrm{x} 165 \mu \mathrm{m}^{2}$

Figure 5-4a shows the DF image of nanoparticles immobilized on glass after the target molecules were incubated and the excess was washed away while Figure 5-4b depicts the same slide at the end of the experiment, after the binding occurred. The image shown in Figure 5-4b contains both specific and non-specific binding of the functionalized $\mathrm{Au}^{40}$ nanoparticles. To determine the LOD two sets of images of the nanoparticles from a specific FOV were recorded: 10 images in the beginning of the experiment (when no changes in the system were added) and 10 images at the end of the experiment (after the excess of developer was washed away).

\subsection{SEM imaging}

To conduct SEM experiments the $\alpha \mathrm{HSA}-\mathrm{Au}^{80}$ particles were immobilized on an in-house manufactured gold-coated substrate.

Gold-coated glass slides (17 mm x $17 \mathrm{~mm} \times 1.1 \mathrm{~mm}$, PGO, D263 T) were used as substrates for the immobilization of AuNPs and their conjugates for SEM measurements. Substrates were cleaned by consecutive washing steps using isopropanol, acetone, ethanol and water and dried in a flow of dry $\mathrm{N}_{2}$. The substrates were then incubated at $4{ }^{\circ} \mathrm{C}$ in a humidity chamber using a $2 \mathrm{mM}$ solution of MUA in a 1:1 mixture of EtOH and MQ water. After incubation, the substrates were thoroughly washed with $\mathrm{EtOH}$ and MQ water and dried in a flow of dry $\mathrm{N}_{2}$. Immediately afterwards, the surface was covered with a 
5. Performance of colorimetric darkfield microscopy in an amplified sandwich immunoassay freshly prepared mixture (0.3 M NHS + 0.04 M EDC) in water and left to incubate for 15 min. The substrates were then washed with water and PBS, immediately incubated with $\alpha$ HSA-Au ${ }^{80}$ conjugates $\left(10^{-13} \mathrm{M}\right)$ for 10 minutes in a humidity chamber and washed with PBS. Subsequently, one half of the substrate was incubated in a solution of HSA $(1 \mu \mathrm{M})$ for 12 hours while the other half of the substrate was incubated in a solution of HRP (1 $\mu \mathrm{M})$ also for 12 hours, incubated at $4^{\circ} \mathrm{C}$ in a humidity chamber. After incubation, all substrates were immersed in a solution of the developer $\left(\alpha \mathrm{HSA}\right.$ - $\left.\mathrm{Au}^{40}\right)$, thoroughly washed with PBS and dried in a flow of dry $\mathrm{N}_{2}$ prior to SEM measurements. The imaging of the samples was done using a Philips XL30 scanning electron microscope.

\subsection{Data analysis}

An in-house Matlab program was used to analyze the images. The main output of this program is the colour parameter of all individual nanoparticles present in the FOV. The colour of the nanoparticle, which is directly dependent on the spectrum of the nanoparticle, is given by the $\mathrm{r} / \mathrm{g}$ ratio, defined as the ratio between the intensities of the red and the green channel of the camera. The spectral calibration of the $\mathrm{r} / \mathrm{g}$ parameter was carried out using a fiber spectrometer. This was done by measuring the spectra of individual nanoparticles in solutions with various glucose concentrations, and thus with various refractive indices. At each refractive index step, the spectra of multiple individual nanoparticles (all with the same spectral information) were acquired. According to the calibration curves, a change of 0.1 in the $\mathrm{r} / \mathrm{g}$ value corresponds to a peak shift of $5 \mathrm{~nm}$ [30].

The procedure for the analysis consists of several steps.

- The flow assay. First, all particle positions are determined from a control image.

We defined the image preceding any change in the system as control. Then a background subtraction is carried out for all images. A number of criteria (chapter 3 , Section 2.5) are set (area, roundness and $\mathrm{r} / \mathrm{g}$ of the nanoparticles) to select the particles of interest and for the selected particles $\mathrm{r} / \mathrm{g}$ was monitored for each of 
5. Performance of colorimetric darkfield microscopy in an amplified sandwich immunoassay the consecutive images. The output of the analysis program contains the position, intensities of the colour channels and the $\mathrm{r} / \mathrm{g}$ of all selected particles in time.

- For the LOD experiment, the "subtraction approach" was used. This procedure provides a quick estimate of the total number of particles that had at least one other particle binding to it. For this method, two images are needed: a control image, taken at the beginning of the experiment and one image at the end of the experiment. After all particles have been localized, a background correction of the two images is performed. The same criteria as in the main program are used to select the particles of interest and for those particles, the value of the $\mathrm{r} / \mathrm{g}$ from the final image is subtracted from the initial. Then limits are set $(0.1<\Delta \mathrm{r} / \mathrm{g}<$ 0.5) and only those particles are counted that experienced a change of the $\mathrm{r} / \mathrm{g}$ within those limits. These limits were set based on the combination between the calibration curves and simulations, in order to eliminate false positives (particles that show a change not originating from bindings).

\section{Results}

\subsection{Validation of the activity of the conjugated nanoparticles}

In order to verify the activity of the proteins on the nanoparticle conjugates a simple bulk assay was designed. In a low-bind Eppendorf tube, containing $100 \mu$ l solution of conjugate a $50 \mu \mathrm{l}$ solution of target molecules was added and left to incubate for 30 minutes. In the case of a positive response a change in color of the mixed solution is expected, which is a sign of aggregation of the conjugates due to their binding to the target molecules. The control, consisting of non- binding target molecules, should not result in a change of colour of the conjugate solution.

\begin{tabular}{l|l|l|l|l|l|l|l} 
agoat & $\begin{array}{l}\text { Goat } \\
\text { IgG-Ab }\end{array}$ & HRP & BSA & $\begin{array}{l}\text { BSA- } \\
\text { biotin }\end{array}$ & HSA & $\begin{array}{l}\alpha \mathbf{H S A}- \\
\mathbf{A u}^{80}\end{array}$ & $\begin{array}{l}\alpha \mathbf{H S A}- \\
\mathbf{A u}^{\mathbf{4 0}}\end{array}$ \\
\hline $1 \mu \mathrm{M}$ & $1 \mu \mathrm{M}$ & $1 \mu \mathrm{M}$ & $1 \mu \mathrm{M}$ & $1 \mu \mathrm{M}$ & $1 \mu \mathrm{M}$ & $10^{-14} \mathrm{M}$ & $10^{-13 \mathrm{M}}$
\end{tabular}

Table 5-1. The concentrations of the materials used in the bulk assay 
5. Performance of colorimetric darkfield microscopy in an amplified sandwich immunoassay

Here we tested the activity of the $\alpha \mathrm{HSA}-\mathrm{Au}^{80}$ and $\alpha \mathrm{HSA}-\mathrm{Au}^{40}$ conjugates using as control agoat, Goat IgG Ab, HRP, BSA and BSA-biotin and HSA as sample (Table 5-1). Two aspects were investigated: (1) the colour and (2) the position of the PP peak of the solutions.

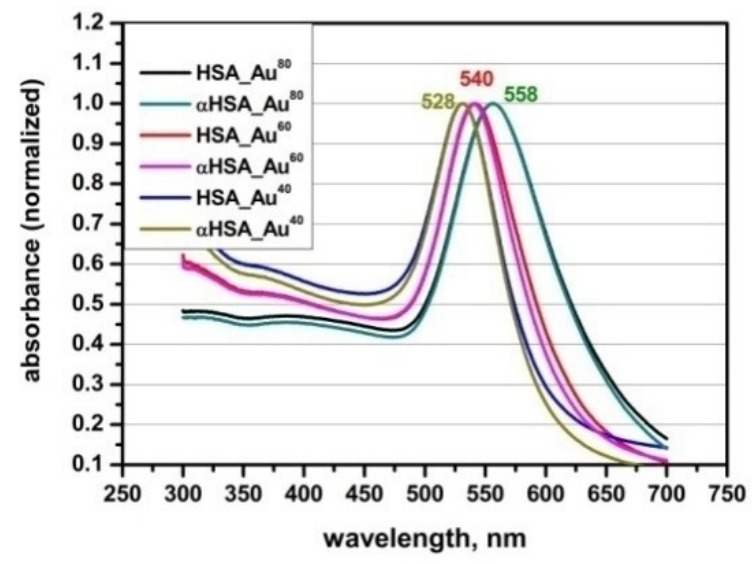

Figure 5-5 The absorption spectra of the conjugates (in bulk) used in the sandwich assay.

(a)

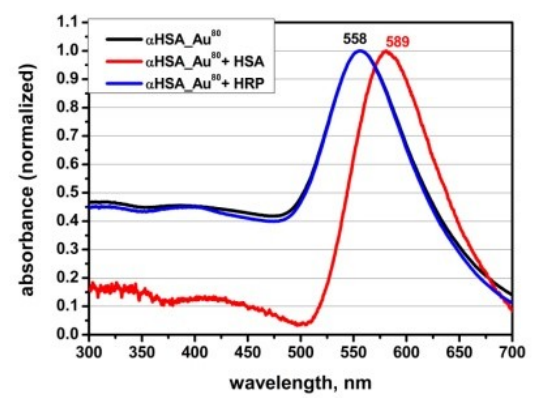

(b)

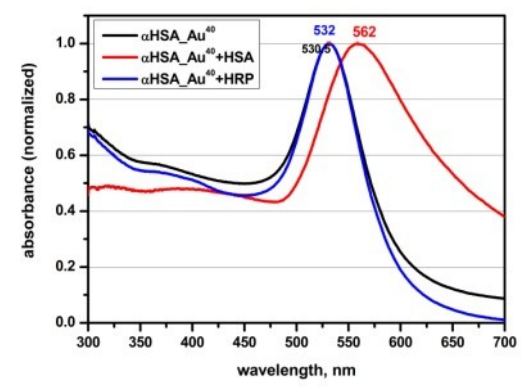

Figure 5-6 The normalized absorption spectra of the (a) $\alpha \mathrm{HSA}-\mathrm{Au}^{80}$ and (b) $\alpha \mathrm{HSA}-\mathrm{Au}^{40}$ conjugates. A wavelength shift is expected when the partner molecule is added in solution. The control experiments show no shifts when a non-interacting protein is added into the conjugates solution.

The results of the colour test provided evidence that aggregation occurs only when specific target molecules (HSA) are added in solution, while when control molecules are added the colour of the solution remained unaltered. To verify the stability of the 
5. Performance of colorimetric darkfield microscopy in an amplified sandwich immunoassay conjugates in the buffer used during the flow-assay, we changed the buffer in which the conjugates were stored and replaced it with PBS. The stability of these conjugates was monitored for 2 weeks. The colour of the conjugate's solutions in PBS remained unaltered. All results presented above establish that the aggregation due to specific binding occurred in the two examined solutions.

The second aspect in establishing the activity of the used conjugates was to monitor the spectral shifts of the above-mentioned mixture solutions. This was accomplished by measuring the position of the PP of the conjugates before and after the analyte molecules were added. Figure 5-5 depicts the absorption spectra of the conjugates used at the beginning of the experiment. Each set of conjugated particles, has its own specific position of the PP, given by the size of the particle and the nature of the receptor molecule conjugated to them.

The graphs presented in Figure 5-6a and Figure 5-6b show the outcome for $\alpha \mathrm{HSA}-\mathrm{Au}^{80}$ and $\alpha \mathrm{HSA}-\mathrm{Au}^{40}$ conjugates. A large wavelength shift can be observed when the specific molecules were added in solution and no shift is apparent when the control molecules were added.

In conclusion, these experiments give a good indication on the activity of the proteins present on the nanoparticles. An SPR immunoassay experiment was also performed on the same conjugates that confirm our bulk results (data not shown).

\subsection{DF results: detection of individual binding events in a sandwich assay}

Next, we present the DF results of the:

- (1) sample sandwich immunoassay: the binding of the anti-human serum albumin functionalized Au40 $\left(\alpha \mathrm{HSA}-\mathrm{Au}^{40}\right)$ to one of the binding sites of the HSA molecules $(1 \mu \mathrm{M})$ bound to the receptor molecules on the immobilized nanoparticle and 
5. Performance of colorimetric darkfield microscopy in an amplified sandwich immunoassay

- (2) the control experiment, that is, the lack of binding of the HRP molecules to the $\alpha \mathrm{HSA}-\mathrm{Au}^{80}$ leading to no binding of the $\alpha \mathrm{HSA}-\mathrm{Au}^{40}$ developer particles.

Figure $5-7 \mathrm{a}$ shows the time traces of 2 individual $\alpha \mathrm{HSA}-\mathrm{Au}^{80}$ particles from the sandwich immunoassay ( $\alpha \mathrm{HSA}-\mathrm{Au}^{80}-\mathrm{HSA}$ - $\alpha \mathrm{HSA}-\mathrm{Au}^{40}$ ) showing a step-like increase in the signal (i.e. in $\mathrm{r} / \mathrm{g}$ ) during the $\alpha \mathrm{HSA}$ - $\mathrm{Au}^{40}$ incubation period. These increases are independent from one another, as they appear at different time stamps at each nanoparticle. The magnitude of these signal changes is much larger than the noise levels and is stable after washing.
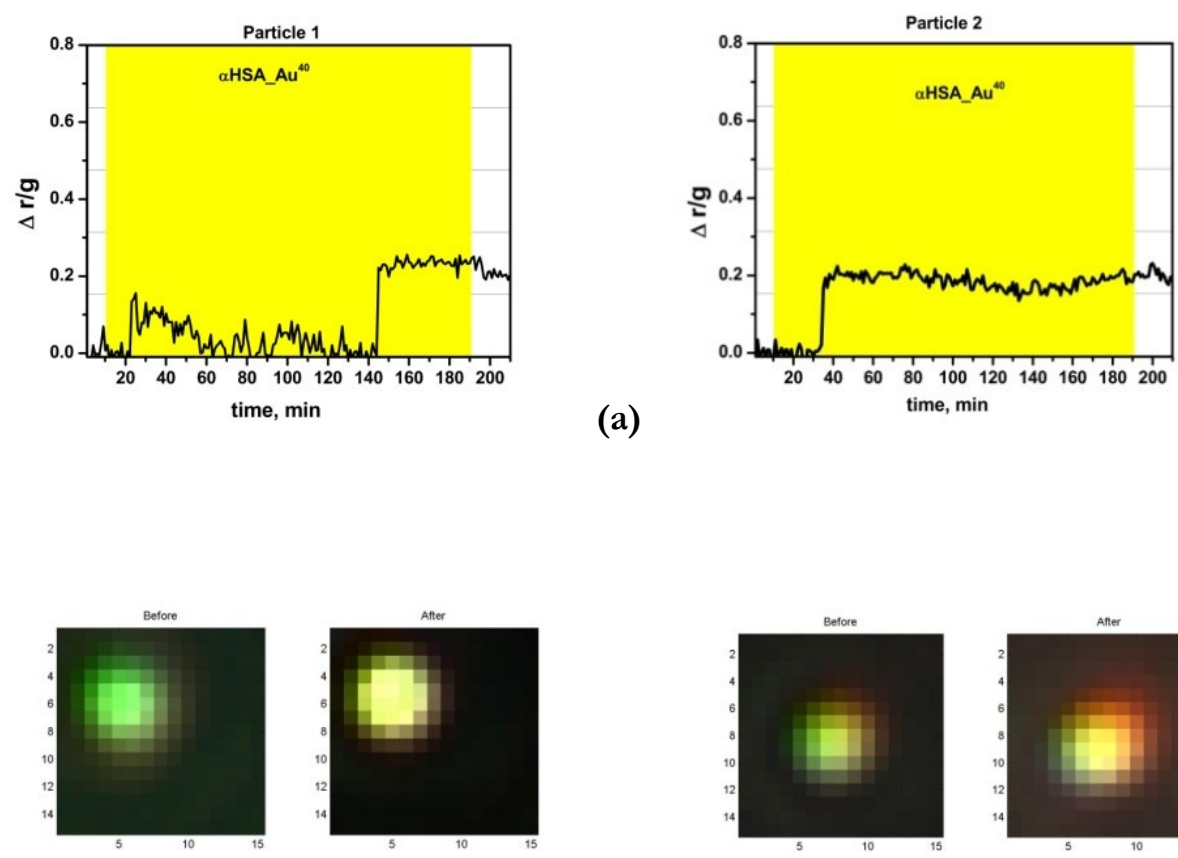

(a)

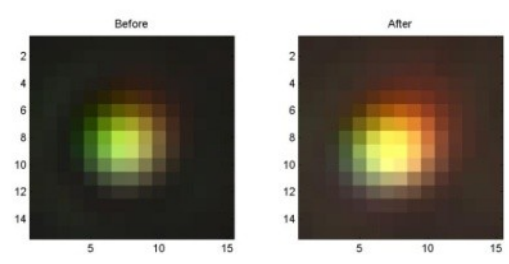

(b)

Figure 5-7 (a) The change in the signal in time for two individual $80 \mathrm{~nm}$ Au particles (b) DF image selection depicting the particle at the beginning and at the end of the experiment

During the washing steps (white regions), the noise levels are between $\Delta \mathrm{r} / \mathrm{g}$ $=0.015-0.025$ while during the incubation time (yellow region) the noise levels increase and a larger fluctuation of the signal can be observed. As discussed in chapter 4, Section 
5. Performance of colorimetric darkfield microscopy in an amplified sandwich immunoassay 3.1, we assume that this increase in the noise levels is the result of the free diffusion of the conjugates that are temporarily approaching the immobilized conjugates.

By correlating the obtained $\mathrm{r} / \mathrm{g}$ changes with the calibration data, we can estimate that these results correspond to an approximately $10 \mathrm{~nm}$ shift, which is in good correlation with the theoretical data. Figure $5-7 \mathrm{~b}$ depicts crops from the DF images of the same particles at the beginning and at the end of the experiment, indicating a change of the colour of the nanoparticle. The colour asymmetry observed in Figure $5-7 \mathrm{~b}$ is a consequence of a chromatic aberration of the microscope caused by a small misalignment between the objective used and the optical path of the microscope. This effect is more visible at the edges of the DF image.

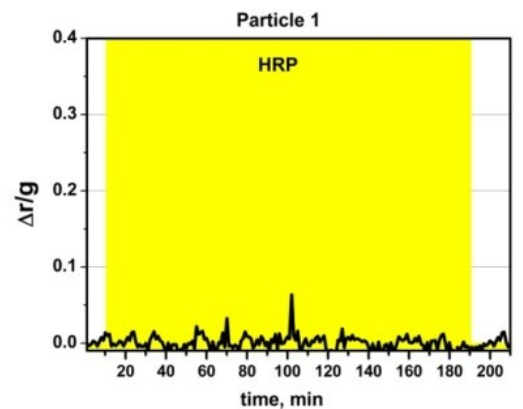

Particle 1

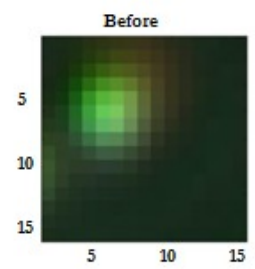

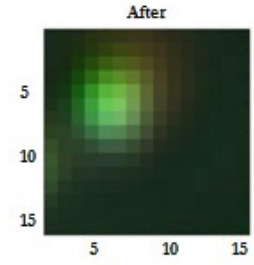

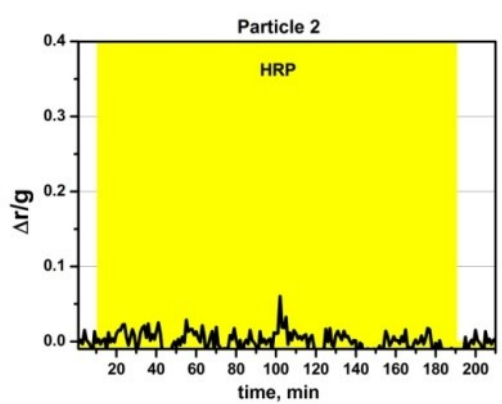

(a)

Particle 2
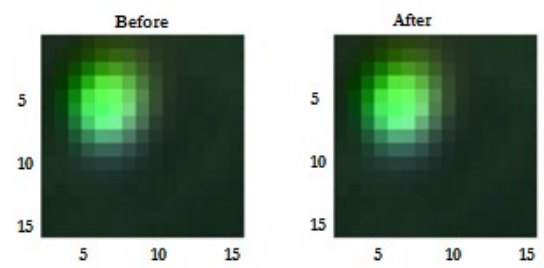

(b)

Figure 5-8 (a) The change of the signal in time of two individual nanoparticles in the control experiment. (b) The corresponding DF crops of the presented particles at the beginning and at the end of the experiment. 
5. Performance of colorimetric darkfield microscopy in an amplified sandwich immunoassay

As control experiment, we monitored the lack of binding of the developer particles $\left(\alpha \mathrm{HSA}-\mathrm{Au}^{40}\right)$ when HRP $(300 \mathrm{nM})$ was used as target molecule. The testing conditions were identical to those in the sample experiment. Figure 5-8a depicts two time traces of two individual particles. A number of similarities were observed between the control and sample signal traces: the noise level during washing was lower $(\Delta \mathrm{r} / \mathrm{g}=0.01$ 0.02 ) and increased to $\Delta \mathrm{r} / \mathrm{g}=0.025-0.05$ during the incubation of HRP-Au ${ }^{40}$. Figure 5-8b illustrates DF pictures of the same particle in the beginning and at the end of the experiments showing no colour change of the investigated particles.

\subsection{Statistics on the $\mathrm{r} / \mathrm{g}$ change- sample and control}

In order to get an impression on the binding process in the sample and control a statistical test was performed. First, the r/g change of all individual nanoparticles from the sample and control was determined at the end of the experiment. A histogram of the final $\mathrm{r} / \mathrm{g}$ change of the particles from sample and control experiments is illustrated in Figure 59, where the vertical scale denotes the fraction of nanoparticles that exhibit a certain $\Delta \mathrm{r} / \mathrm{g}$. The bin size was set to 0.05 . Due to the differences in the number of nanoparticles present in the FOV in the sample and control experiments, a normalization of the population was performed.

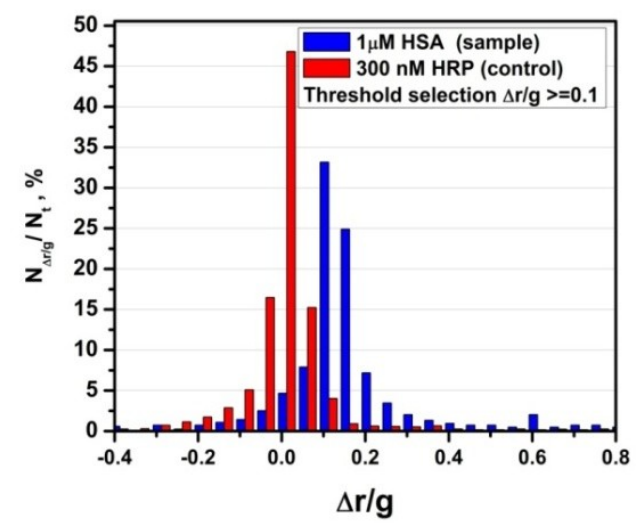

Figure 5-9 Comparison between the colour change distribution between the sample and the control. 
5. Performance of colorimetric darkfield microscopy in an amplified sandwich immunoassay

The population of the control experiment is clustered around $\Delta \mathrm{r} / \mathrm{g}=0$, representing no change in the spectral properties of the nanoparticle. In the experiment where specific target molecules were added, the most frequent $\Delta \mathrm{r} / \mathrm{g}$ is $0.1-0.2$, suggesting that binding events occurred. By eliminating all the particles that fall out of the threshold range $(0.1<\Delta \mathrm{r} / \mathrm{g}<0.5)$ we observe that, in the sample, $50 \%$ of the present particles $\left(\mathrm{N}^{\text {total }}=849\right)$ show a positive $\mathrm{r} / \mathrm{g}$ change above the threshold while in the control only $5 \%$ of the analyzed particles $\left(\mathrm{N}^{\text {total }}=928\right)$ showed an observable steplike increase.

\subsection{SEM results}

For the confirmation of the DF results, we performed a SEM experiment, results of which are shown in Figure 5-10. The $80 \mathrm{~nm}$ Au particles can be easily differentiated from the $40 \mathrm{~nm}$ particles, as they look bigger while the particle clusters are even easier to recognize. Clusters of $\mathrm{Au}^{80}-\mathrm{Au}^{40}$ can be distinguished in Figure 5-10a while no such aggregates are present in the control experiment (Figure 5-10b), representing a clear indication of specific binding process in the sample experiment.

In the case of the sample (Figure 5-10a) the number of $40 \mathrm{~nm}$ particles adsorbed to the surface is larger than in the case of the control (Figure 5-10b). Because in these SEM experiments the surface was not previously passivated, the non-specific adsorption of the target molecules (HSA or HRP) on the surface could not be prevented. Therefore, in the control the HRP molecules act as a passivating agent, blocking the binding of the $\alpha \mathrm{HSA}-\mathrm{Au}^{40}$ to the surface. In the sample however, the binding of the $\alpha \mathrm{HSA}-\mathrm{Au}^{40}$ to the surface is favoured for two reasons: (1) the APTES coated surface is known for its "sticky" nature and (2) the non-specifically adsorbed HSA molecules on the surface are extra receptor sites available for the $\alpha \mathrm{HSA}-\mathrm{Au}^{40}$ nanopaticles. However, this is not an impediment, for two reasons: (1) we can easily distinguish between the 3 population of particles present on the sensor's surface: $80 \mathrm{~nm}$ particles, $40 \mathrm{~nm}$ particles and $80-40 \mathrm{~nm}$ complexes and (2) we are interested in visualizing the $80-40 \mathrm{~nm}$ aggregates. 

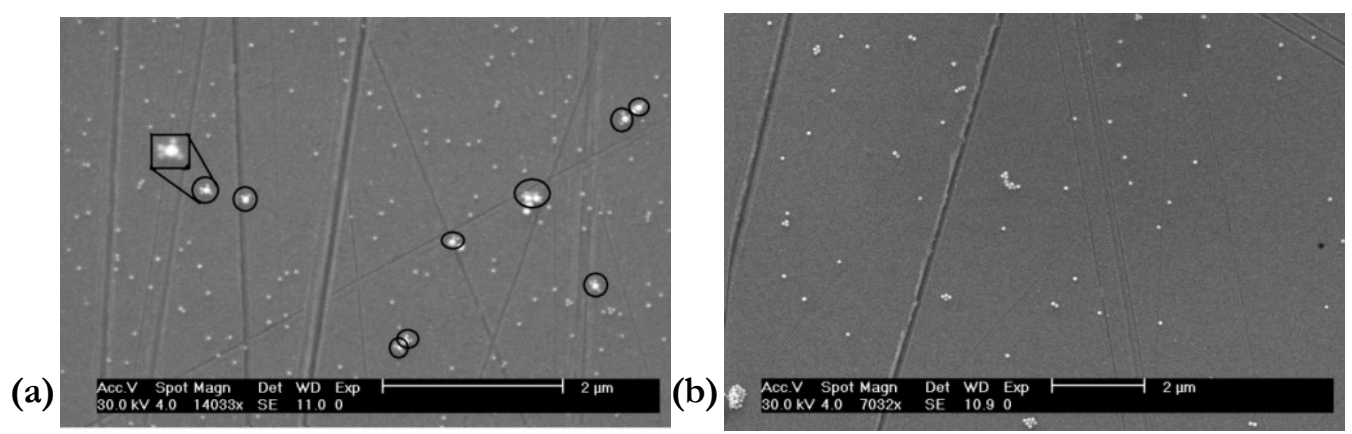

Figure 5-10 SEM images of the sample (a) and control immunoassay (b). The $80 \mathrm{~nm}$ particles with at least one $40 \mathrm{~nm}$ particle binding to them are tagged with black circles. The inset in the sample image shows a zoom of the $80 \mathrm{~nm}$ functionalized particle with a few bound $40 \mathrm{~nm}$ conjugated particles.

We determined the fraction of the $80 \mathrm{~nm}$ particles having $40 \mathrm{~nm}$ particles bound to them from the SEM images as $0.40\left(\mathrm{~N}^{\text {total }}=280\right)$ in the case of the sample and 0.01 $\left(\mathrm{N}^{\text {total }}=201\right)$ in the case of the (negative) control.

These results were directly compared with the DF data where we determined the fraction of the $80 \mathrm{~nm}$ particles having at least one $40 \mathrm{~nm}$ particles bound to them using a subtraction program (cf. chapter 3, Section 2.5). Here, the last image of the DF experiment was subtracted from the initial image and only the particles that showed a change were counted, as shown in Figure 5-11.

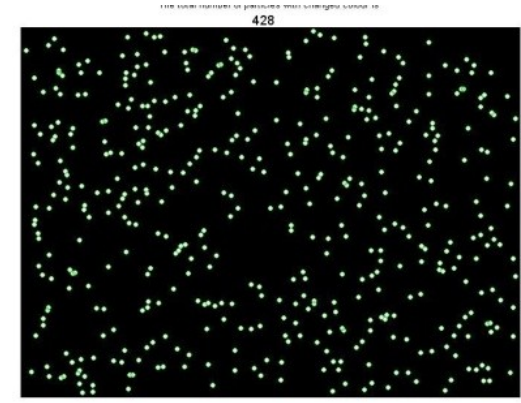

(a)

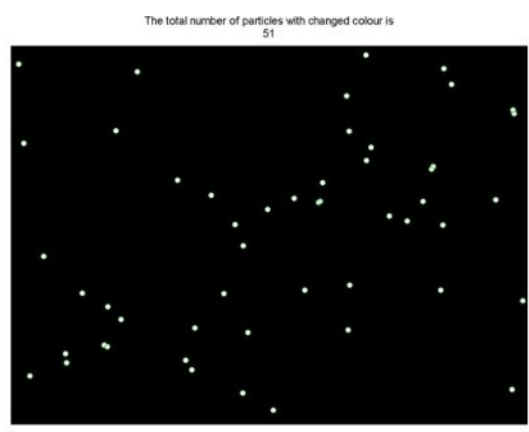

(b)

Figure 5-11 The images of the selected particles after using the selection algorithm in the (a) sample and (b) control experiment 
5. Performance of colorimetric darkfield microscopy in an amplified sandwich immunoassay

\begin{tabular}{l|cccc}
\hline & \multicolumn{2}{|c}{ DF } & \multicolumn{2}{c}{ SEM } \\
& Sample & control & Sample & control \\
\hline Bound & 428 & 51 & 112 & 2 \\
Unbound & 421 & 877 & 168 & 199 \\
Binding fraction (\%) & 50.4 & 5.5 & 40 & 0.99 \\
Total & 849 & 928 & 280 & 201
\end{tabular}

Table 5-3. Comparison between DF and SEM experiments presenting the statistics over the binding fraction of the sample and control experiments

Binding fractions (Table 5-3) obtained from SEM (0.4 for sample, respectively 0.01 for control) data are in good agreement with those obtained from darkfield experimental data ( 0.5 for sample, respectively 0.05 for control).

\subsection{LOD of the system}

Next, we evaluated the sensitivity of our detection method for the protein immunoassay application, by incubating different concentrations of target molecules over the immobilized $\mathrm{Au}^{80}$ conjugates.

Four flow-cells (as described in chapter 3, Section 3.3) were used totalizing 12 channels. Eight were incubated with different target concentrations ranging from $1 \mu \mathrm{M}$ to $1 \mathrm{nM}$, and three channels were incubated (with molecules, which should not bind to the functionalized particles, in various concentrations from $0.5 \mu \mathrm{M}$ to $1 \mathrm{nM}$ ). One channel was not used due to imperfections in the construction of the channel. For the reproducibility of the data, at each target concentration, three different sensing areas from that channel were measured.

Before the introduction of the target molecules in the channel, a DF image was acquired. After the incubation of the target molecules, the flowcells were washed and a second DF image was acquired. For data analysis, the subtraction algorithm was used as described before. 
5. Performance of colorimetric darkfield microscopy in an amplified sandwich immunoassay

The experiment was conducted as follows:

- The sensing surfaces were washed with PBS buffer for 30 minutes (flow rate=14 $\mu \mathrm{l} / \mathrm{min})$.

- The target was incubated for 2 hours (no flow).

- The excess was washed for 10 minutes with PBS buffer (flow rate $=14 \mu \mathrm{l} / \mathrm{min}$ )

- The developer $\left(\alpha \mathrm{HSA}-\mathrm{Au}^{40}\right)$ was incubated for 3 or 4 hours (no flow). The incubation of the developer was performed in a target-free environment to avoid the growth of developer aggregates in solution.

- To remove the non-bound developer particles the sensor was washed for 20 minutes.

The consequence of working in a target-free environment is that the target molecules that dissociates naturally from the receptor molecules cannot be replaced with new molecules from the solution. As a result, the $\alpha \mathrm{HSA}-\mathrm{Au}^{40}$ particles will have less and less binding sites available. The remaining fraction of bound target molecules after dissociation is dependent on the dissociation constant and the incubation time of the developer in a target-depleted medium [38]. By considering the dissociation rate of the HSA- $\alpha$ HSA complex $k_{\mathrm{d}}=3.92 * 10^{-5} \mathrm{~s}^{-1}[35]$ and the time spent in a target-depleted environment, the remaining fraction of the target molecules originally bound at the receptor molecules can be estimated $(0.3 \%$ for 3 hours incubation time, respectively $0.1 \%$ for 4 hours). The effects of this process on the performance of our detection method can be observed in Figure 5-12.

Here, we plotted the coverage as a function of concentration for two incubation times of the developer in a target-depleted solution: 3 hours (black squares) and 4 hours (red circles). A dramatic decrease of the response is observed when the incubation time of the developer is increased: for example, at $1 \mu \mathrm{M}$ introduced target concentration the coverage $\Gamma$ at 3 hours incubation was 0.45 , while at 4 hours incubation the coverage was a factor 3 lower. In addition, at higher incubation times of the developer the minimum detectable concentration (LOD) was worsened. The results presented here indicate that the dissociation process has important implications for the performance of our approach. 


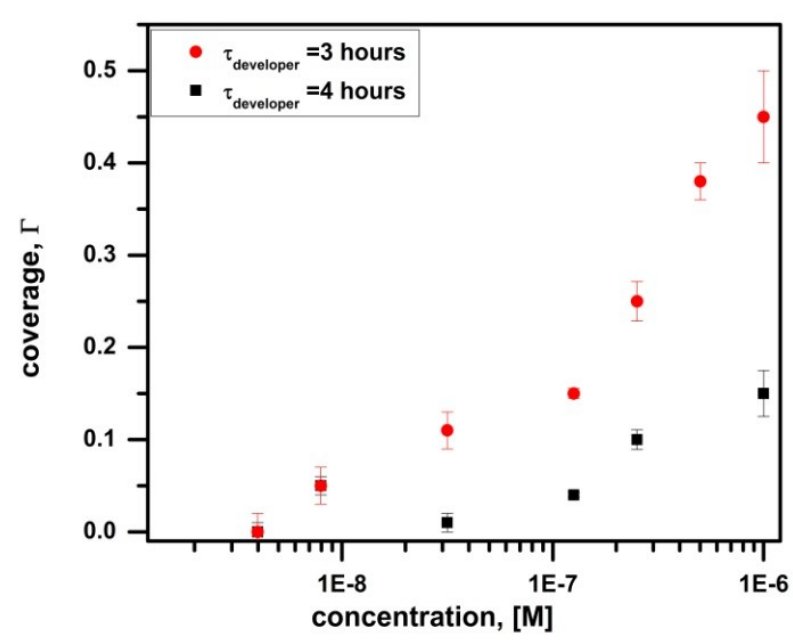

Figure 5-12 The immunoreaction response as a function of the HSA concentration with the developer incubation time $(\tau)$ as a parameter.

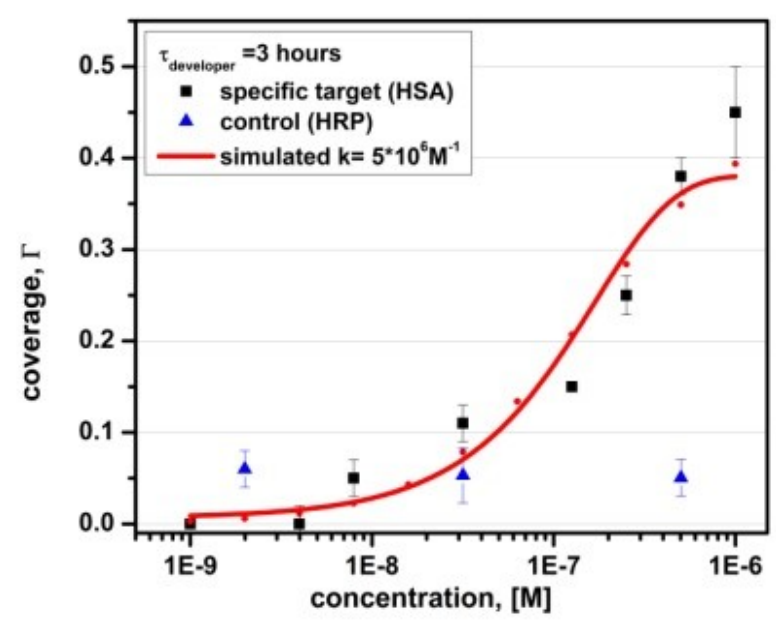

Figure 5-13 The concentration dependence of the response based upon the fraction of Au nanoparticles that changed colour after the incubation of the developer.

To determine the LOD of our method we have plotted the immunoresponse as a function of concentration, as depicted in Figure 5-13. The response is calculated by counting the fraction of $\mathrm{Au}$ nanoparticles that change their colour due to binding at 
5. Performance of colorimetric darkfield microscopy in an amplified sandwich immunoassay different concentrations. Here, the results of the concentration dependence of the response for specific binding (black squares) and control (blue triangles) were plotted. The response in the specific case varies from 0.45 to 0.01 while the control shows a low response $(0.05)$ over the whole concentration range.

For the fitting of the experimental data shown in Figure 5-13 (black squares) we performed a Monte Carlo (MC) simulation (Nparticles $=1000 ;$ Nprotein $/$ particle $=200$; $\mathrm{k}=5^{*} 10^{6} \mathrm{M}^{-1}$, described in chapter 2 , Section 5.2. In this fit, we took into account that due to dissociation only $0.03 \%$ of the original bound targets were available for binding to the developer conjugate. With this correction added, a reasonable agreement can be observed between the theoretical (red curve) and experimental data (black squares).

According to these experimental results, the LOD obtained here is around 30 $\mathrm{nM}$. According to the MC simulations, for this type of immunoassay, if no depletion were present, we would expect the LOD to be in the picomolar range.

\section{Conclusions}

In this chapter, we have shown that a simple DF setup and our detection methodology can be used to detect sandwich formation in a protein immunoassay. The method presented above can be used in two modes:

- The time monitoring of the binding process. This mode gives a good insight on the binding process indicating details such as the time a binding event occurred or the number of binding events per particle. This approach can be an interesting tool to study biochemical reactions or to obtain insight in the antibody binding behaviour.

- The difference method. Here, the response of the sensor is calculated by taking the difference between the initial and final state of the particles within the sensing area and counting all those particles that show a colour change between certain limits. In this approach, the details on the binding process per particle are lost. This mode is advantageous as it gives a rapid indication on the amount of particles that suffered a change in its state due to binding. However, in this approach, more caution in data interpretation is necessary, and the control experiment is almost mandatory. 
5. Performance of colorimetric darkfield microscopy in an amplified sandwich immunoassay

A comparison of the sensitivities of various detection strategies of biomolecules with our method is presented in Table 5-4.

\begin{tabular}{|c|c|c|c|}
\hline Method & Type & LOD & Refference \\
\hline ELISA & $\begin{array}{l}\text { Antigen } \\
\text { Antibody } \\
\text { Antibody-enzime }\end{array}$ & $0.1 \mathrm{nM}$ & $\begin{array}{l}\text { Dixit et al } \\
(2010)[36]\end{array}$ \\
\hline SPR & $\begin{array}{l}\text { Antigen } \\
\text { Antibody }\end{array}$ & $1 \mathrm{nM}$ & $\begin{array}{l}\text { Lee et al. } \\
(2006)[37]\end{array}$ \\
\hline $\begin{array}{l}\text { Colourimetric } \\
\text { method using Au } \\
\text { Np's }\end{array}$ & $\begin{array}{l}\text { AuNP-ODN receptor } \\
\text { DNA target } \\
\text { AuNP-ODN }\end{array}$ & $50 \mathrm{pM}$ & Storhoff et al. [12] \\
\hline $\begin{array}{l}\text { Un-amplified } \\
\text { assay } \\
\text { Nanoparticles }\end{array}$ & $\begin{array}{l}\text { Au Np-antibody } \\
\text { receptor } \\
\text { Antigen target }\end{array}$ & $10 \mathrm{nM}$ & Chapter 3 \\
\hline $\begin{array}{l}2 \text { Au NP's } \\
\text { proximity } \\
\text { Parallel detection }\end{array}$ & $\begin{array}{l}\mathrm{Au} \quad \mathrm{Np} \text {-antibody } \\
\text { receptor } \\
\text { Antigen target } \\
\mathrm{Au} \quad \mathrm{Np} \text {-antibody } \\
\text { receptor }\end{array}$ & $10 \mathrm{nM}$ & This study \\
\hline $\begin{array}{l}2 \text { Au NP's } \\
\text { proximity } \\
\text { Parallel detection }\end{array}$ & $\begin{array}{l}\mathrm{Au} \quad \mathrm{Np} \text {-antibody } \\
\text { receptor } \\
\text { Antigen target } \\
\mathrm{Au} \quad \mathrm{Np} \text {-antibody } \\
\text { receptor }\end{array}$ & $0.35 \mathrm{pM}$ & $\begin{array}{l}\text { Chen et al. (2011) } \\
\text { [20] }\end{array}$ \\
\hline $\begin{array}{l}2 \mathrm{Au} \text { Np's } \\
\text { proximity } \\
\text { Parallel detection }\end{array}$ & $\begin{array}{l}\text { AuNP-ODN receptor } \\
\text { DNA target } \\
\text { AuNP-ODN }\end{array}$ & $10 \mathrm{fM}$ & $\begin{array}{l}\text { Verdoold et al. } \\
\text { (2011) [38] }\end{array}$ \\
\hline
\end{tabular}

Table 5 - 4. The sensitivities of various detection approaches for biomolecules 
5. Performance of colorimetric darkfield microscopy in an amplified sandwich immunoassay

The experimental sensitivity of our sensing method, in the protein immunoassay is in the nanomolar range, and is somewhat disappointing. However, the low detection limit obtained in the detection of DNA molecules using this approach [38], and the finding that in our current experiments the occurrence of depletion plays such a decisive role makes us believe that the performance of this approach in the protein assay may be substantially improved.

Future experiments on this assay should be carried out with a focus on shortening the assay time, thereby avoiding depletion effects, and on the minimization of non-specific interactions. In addition, the number of receptor antibodies on an $\mathrm{Au}^{80} \mathrm{np}$ should be optimized, and the number of immobilized nanoparticles in the FOV should be maximized, e.g., by immobilizing them in an ordered array, using cleanroom techniques. 


\section{References}

[1] B. Perez-Lopez and A. Merkoci, "Nanomaterials based biosensors for food analysis applications," Trends in Food Science \& Technology, vol. In Press,.

[2] J.-in Hahm, "Functional Polymers in Protein Detection Platforms: Optical, Electrochemical, Electrical, Mass-Sensitive, and Magnetic Biosensors," Sensors, vol. 11, 2011, pp. $3327-3355$.

[3] J. Homola, "Present and future of surface plasmon resonance biosensors," Analytical and Bioanalytical Chemistry, vol. 377, pp. 528-539.

[4] M. Willander and S. Al-Hilli, “Analysis of Biomolecules Using Surface Plasmons,” Methods in Molecular Biology, vol. 544, 2009, pp. 201-229.

[5] Y. Liu and D.W. Wilson, "Quantitative Analysis of Small Molecule-Nucleic Acid Interactions with a Biosensor Surface and Surface Plasmon Resonance Detection," Methods in Molecular Biology, vol. 613, 2010, pp. 1-23.

[6] R. D'Agata, R. Corradini, C. Ferretti, L. Zanoli, M. Gatti, R. Marchelli, and G. Spoto, "Ultrasensitive detection of non-amplified genomic DNA by nanoparticle-enhanced surface plasmon resonance imaging," Biosensors and Bioelectronics, vol. 25, May. 2010, pp. 2095-2100.

[7] W. Lian, S.A. Litherland, H. Badrane, W. Tan, D. Wu, H.V. Baker, P.A. Gulig, D.V. Lim, and S. Jin, "Ultrasensitive detection of biomolecules with fluorescent dye-doped nanoparticles," Analytical Biochemistry, vol. 334, Nov. 2004, pp. 135-144.

[8] K.A. Fahnrich, M. Pravda, and G.G. Guilbault, "Recent applications of electrogenerated chemiluminescence in chemical analysis," Talanta, vol. 54, May. 2001, pp. 531-559.

[9] U. Resch-Genger, M. Grabolle, S. Cavaliere-Jaricot, R. Nitschke, and T. Nann, "Quantum dots versus organic dyes as fluorescent labels," Nat Meth, vol. 5, Sep. 2008, pp. 763-775.

[10] C.A. Mirkin, R.L. Letsinger, R.C. Mucic, and J.J. Storhoff, "A DNA-based method for rationally assembling nanoparticles into macroscopic materials," Nature, vol. 382, Aug. 1996, pp. 607-609. 
5. Performance of colorimetric darkfield microscopy in an amplified sandwich immunoassay [11] J. Yguerabide and E.E. Yguerabide, "Light-Scattering Submicroscopic Particles as Highly Fluorescent Analogs and Their Use as Tracer Labels in Clinical and Biological Applications: I. Theory," Analytical Biochemistry, vol. 262, Sep. 1998, pp. 137-156.

[12] J.J. Storhoff, A.D. Lucas, V. Garimella, Y.P. Bao, and U.R. Muller, "Homogeneous detection of unamplified genomic DNA sequences based on colorimetric scatter of gold nanoparticle probes," Nat Biotech, vol. 22, Jul. 2004, pp. 883-887.

[13] W. Fritzsche and T.A. Taton, "Metal nanoparticles as labels for heterogeneous, chipbased DNA detection," Nanotechnology, vol. 14, 2003, p. R63.

[14] A.J. Haes, W.P. Hall, L. Chang, W.L. Klein, and R.P. Van Duyne, "A Localized Surface Plasmon Resonance Biosensor: First Steps toward an Assay for Alzheimer's Disease," Nano Letters, vol. 4, May. 2004, pp. 1029-1034.

[15] K. Glynou, P.C. Ioannou, T.K. Christopoulos, and V. Syriopoulou, "OligonucleotideFunctionalized Gold Nanoparticles as Probes in a Dry-Reagent Strip Biosensor for DNA Analysis by Hybridization," Analytical Chemistry, vol. 75, Jul. 2003, pp. 4155-4160.

[16] A.D. McFarland and R.P. Van Duyne, "Single Silver Nanoparticles as Real-Time Optical Sensors with Zeptomole Sensitivity," Nano Letters, vol. 3, Jul. 2003, pp. 1057-1062.

[17] T. Sannomiya, C. Hafner, and J. Voros, "In situ Sensing of Single Binding Events by Localized Surface Plasmon Resonance,” Nano Letters, vol. 8, Sep. 2008, pp. 3450-3455.

[18] G. Raschke, S. Kowarik, T. Franzl, C. Sönnichsen, T.A. Klar, J. Feldmann, A. Nichtl, and K. Kürzinger, "Biomolecular Recognition Based on Single Gold Nanoparticle Light Scattering," Nano Letters, vol. 3, May. 2003, pp. 935-938.

[19] C.L. Baciu, J. Becker, A. Janshoff, and C. Sönnichsen, "Protein-Membrane Interaction Probed by Single Plasmonic Nanoparticles," Nano Letters, vol. 8, May. 2008, pp. 1724-1728.

[20] S. Chen, M. Svedendahl, R.P.V. Duyne, and M. Kall, "Plasmon-Enhanced Colorimetric ELISA with Single Molecule Sensitivity,” Nano Letters, vol. 11, Mar. 2011, pp. 1826-1830.

[21] F. Ungureanu, J. Halamek, R. Verdoold, and R.P.H. Kooyman, "The use of a colour camera for quantitative detection of protein binding nanoparticles," Proc. SPIE 7192, vol. 71920O, 2009. 
5. Performance of colorimetric darkfield microscopy in an amplified sandwich immunoassay [22] F. Ungureanu, D. Wasserberg, N. Yang, R. Verdoold, and R.P.H. Kooyman, "Immunosensing by colorimetric darkfield microscopy of individual gold nanoparticle-conjugates," Sensors and Actuators B: Chemical, vol. 150, Oct. 2010, pp. 529-536.

[23] P.J. Draine, B.T., \& Flatau, "Discrete dipole approximation for scattering calculations," J. Opt. Soc. Am. A, vol. 11, 1994, pp. 1491-1499.

[24] M.A. Yurkin, D. de Kanter, and A.G. Hoekstra, "Accuracy of the discrete dipole approximation for simulation of optical properties of gold nanoparticles," Journal of Nanophotonics, vol. 4, 2010.

[25] C. Ungureanu, R.G. Rayavarapu, S. Manohar, and T.G. van Leeuwven, "Discrete dipole approximation simulations of gold nanorod optical properties: Choice of input parameters and comparison with experiment," Journal of Applied Physics, vol. 105, 2009, pp. 102032 - 102032-7.

[26] P.B.J. and R.W. Christy, "Optical Constants of the Noble Metals," Physics Review Letters, vol. 6, 1972, pp. 4370-4379.

[27] A.M. Funston, C. Novo, T.J. Davis, and P. Mulvaney, "Plasmon Coupling of Gold Nanorods at Short Distances and in Different Geometries," Nano Letters, vol. 9, Mar. 2009, pp. 1651-1658.

[28] P.K. Jain, S. Eustis, and M.A. El-Sayed, "Plasmon Coupling in Nanorod Assemblies: Optical Absorption, Discrete Dipole Approximation Simulation, and Exciton-Coupling Model," The Journal of Physical Chemistry B, vol. 110, Aug. 2006, pp. 18243-18253.

[29] W. Rechberger, A. Hohenau, A. Leitner, J.R. Krenn, B. Lamprecht, and F.R. Aussenegg, “Optical properties of two interacting gold nanoparticles," Optics Communications, vol. 220, 2003, pp. 137-141.

[30] C. Sonnichsen, B.M. Reinhard, J. Liphardt, and A.P. Alivisatos, “A molecular ruler based on plasmon coupling of single gold and silver nanoparticles," Nat Biotech, vol. 23, Jun. 2005, pp. 741-745.

[31] K.B. Cederquist and C.D. Keating, "Curvature Effects in DNA:Au Nanoparticle Conjugates," ACS Nano, vol. 3, Feb. 2009, pp. 256-260. 
5. Performance of colorimetric darkfield microscopy in an amplified sandwich immunoassay [32] A.A. Vertegel, R.W. Siegel, and J.S. Dordick, "Silica Nanoparticle Size Influences the Structure and Enzymatic Activity of Adsorbed Lysozyme," Langmuir, vol. 20, May. 2004, pp. 68006807.

[33] R.A. Reynolds, C.A. Mirkin, and R.L. Letsinger, "Homogeneous, Nanoparticle-Based Quantitative Colorimetric Detection of Oligonucleotides," Journal of the American Chemical Society, vol. 122, Apr. 2000, pp. 3795-3796.

[34] E. Wedege and G. Svenneby, "Effects of the blocking agents bovine serum albumin and Tween 20 in different buffers on immunoblotting of brain proteins and marker proteins," Journal of Immunological Methods, vol. 88, 1986, pp. 233-237. C. Joo, E. Ozkumur, M.S. Unlu, and J.F. de Boer, "Spectral-domain optical coherence phase microscopy for label-free multiplexed protein microarray assay," Biosensors and Bioelectronics, vol. 25, Oct. 2009, pp. 275-281.

[36] C.K. Dixit, S.K. Vashist, F.T. O’Neill, B. O'Reilly, B.D. MacCraith, and R. O’Kennedy, "Development of a High Sensitivity Rapid Sandwich ELISA Procedure and Its Comparison with the Conventional Approach," Analytical Chemistry, vol. 82, Jul. 2010, pp. 7049-7052.

[37] H.J. Lee, D. Nedelkov, and R.M. Corn, "Surface Plasmon Resonance Imaging Measurements of Antibody Arrays for the Multiplexed Detection of Low Molecular Weight Protein Biomarkers," Analytical Chemistry, vol. 78, Aug. 2006, pp. 6504-6510.

[38] R. Verdoold, R. Gill, F. Ungureanu, and R.P.H. Kooyman, "Femtomolar DNA detection by parallel colorimetric darkfield microscopy of functionalized gold nanoparticles," Biosensors and Bioelectronics, 2011. 
5. Performance of colorimetric darkfield microscopy in an amplified sandwich immunoassay 


\section{Chapter 6}

\section{Conclusions}

Abstract::This chapter evaluates the results obtained in this study and presents further recommendations for future work. 
In this thesis, we have shown the proof of principle of optical detection of biomolecules using gold nanoparticles as sensing elements in a colorimetric darkfield setup. As stated in chapter 1 the ideal biosensor should be sensitive, selective, and small, require low sample volume, and be affordable.

The low cost technology used in this approach is a strong point promoting this method in biosensing applications. However, further work has to be done to increase the sensitivity of the method. The experimental LOD of this approach is disappointing when compared with established technologies such as SPR [1,2]. For example, in label-free immunoassays we obtained an LOD of only $40 \mathrm{nM}$ for HSA molecules, insufficient for practical applications (chapter 3). We believe that the main reason for this lack of sensitivity is the far from optimized surface chemistry. Apart from this aspect, a solution to improve the sensitivity would be to increase the number of available receptor sites in the FOV, as presented in chapter 2, section 5.2.

However, this strategy is not enough to boost the sensitivity. Therefore, an amplification step is required. Theoretically, in this case the sensitivity could be improved with several orders of magnitude (chapter 2, section 5.2). However, the experimental results obtained for HSA molecules (chapter 5, section 3.5) show an LOD in the range of tens of nM, which is insufficient for practical applications. In this case, the combination of high depletion rates and low number of available receptor sites led to poor LOD. Nevertheless, when the same amplification approach was used in DNA sensing applications, the obtained LOD was in the femtomolar range [3], which in our case strongly points to a non-optimum surface chemistry (for possible solutions please see below).

An important factor in measuring low concentrations is the ability to monitor in parallel the signal from multiple individual nanoparticles. In this thesis, we demonstrated the parallelization capabilities of our imaging spectroscopy approach by monitoring the signal of thousands of individual nanoparticles, which is unmatched by current methods like LCTF [4] or AOTF [5]. A high sensitivity to spectral shifts is required to detect binding events. Additionally, a high SNR provides a better accuracy in peak shift determination, which is very important because it provides information about the number 
of protein molecules bound to the particle. For the color camera approach, we have experimentally determined peak shifts as low as $1 \mathrm{~nm}$ (SNR of 4). This peak shift translates into detecting the binding of approximately 50 protein molecules (protein with $6 \mathrm{~nm}$ in diameter on a $60 \mathrm{~nm}$ spherical gold nanoparticle). In the amplified approach, each binding event usually generates a peak shift larger than $1 \mathrm{~nm}$ (chapters $4 \& 5$ ), which is easily detected by our setup.

In the search of the best detection approach, we compared the color camera with two monochrome cameras with custom filters in front (chapter 3, section 4). We expected that this latter approach would provide better spectral sensitivity to spectral changes and higher SNR. Nevertheless, the results of the comparison showed that the color camera is a better option. It is worth mentioning that in this study a dedicated color camera was used; at this moment, we do not know how the performance of a low-cost color camera would affect our results.

Another important component in our sensing approach is the analysis software. This software translates the changes in color of the particles recorded by the camera into a quantified value (e.g. number of proteins, binding events).

The results determined with this software correlate well with theoretical predictions. However, low SNR (induced by low light levels), focus drift and noise (e.g. impurities from the solution adsorbing on or near the nanoparticles, nanoparticles washed away, etc) may alter the outcome of analysis. The focus drift was controlled by using a feedback-motorized stage for the probe. The other perturbing factors were kept to a minimum by using clean buffers and sensor slides.

A correct interpretation of the sensor response relies upon the full understanding of the observed PP shifts under various situations like different polarization states. Theoretically, we showed in chapter 2, section 5.2 that aggregates under different polarization states display different localizations of the PP. However, in the DF approach the polarization states are limited to in-plane variation. Therefore, this aspect could be properly investigated using a total internal reflectance setup where both parallel and perpendicular polarization states can be selected. 
The outcome from chapters 4 and 5 , and in particular from chapter 5 , revealed that the following areas require further attention:

Conjugation of the gold nanoparticles. Ideally, the conjugated nanoparticles should fulfill the following requirements:

- Optimal surface density of antibodies to avoid steric hindrance;

- The right orientation of the antibodies such that the access to the active sites of the antibody is not hindered;

- Stability in time of the conjugated nanoparticles with a long shelf time with no major loss in activity of the antibodies.

The current strategy uses covalent binding of the antibody to the surface of the gold nanoparticles via a linker. The advantage of the method is that it offers a stable layer of antibodies on the nanoparticles. However, the result is a layer of antibodies that are randomly oriented. For example, the surface density of receptors can be increased by immobilizing only the Fab fragments of the antibodies containing the receptor sites, by targeting the thiol groups of the fragments [6]. These Fab fragments are usually obtained by enzymatic cleavage. Alternatively, one could consider using camelid antibodies or nanobodies [7], which are significantly smaller than standard antibodies.

Additionally, the shelf time of the conjugates is rather short $(\sim$ in the order of weeks). Therefore, these areas need to be further optimized.

- Assay time. Further work should be carried out on decreasing the total assay time. Essentially, the immunoreaction is a very fast process. The limiting factor is the diffusion coefficient of the conjugated nanoparticles, which can be estimated from the following relation:

$$
X^{2}=2 D t \text { where } D=\frac{R T}{6 \pi \rho r N}
$$

where $\mathrm{D}$ is the diffusion coefficient of a molecule/nanoparticle with radius $\mathrm{r}$ moving in a solution of viscosity $\varrho . \mathrm{R}$ is the gas constant, $\mathrm{N}$ the Avogadro number and $\mathrm{T}$ the absolute temperature. $\mathrm{X}$ estimates the distance that a 
nanoparticle will travel in the time t. Considering a $60 \mathrm{~nm}$ Au particle in water at room temperature, in 1 minute it travels only $19 \mu \mathrm{m}$. In order to improve the assay time, the flowcell must be optimized by creating a smaller sample chamber Additionally, lower assay times can be obtained if the developer nanoparticles are forced towards to the interface e.g. by using magnetic core gold particles controlled by an external magnetic field).

- Optimization of the number of nanoparticles in the FOV. Throughout this thesis, the nanoparticles were randomly immobilized on the surface of the sensor leading to inefficient use of the available surface area. A possible solution is to immobilize them in an ordered array, for example by using nanolithography [8]. For example, approximately $4 * 10^{4}$ nanoparticles $(80 \mathrm{~nm}$ diameter), at $1 \mu \mathrm{m}$ interparticle distance could be placed in the FOV of 230x170 $\mu \mathrm{m}^{2}$.

- Data Analysis. Further work is needed to find the best strategy to minimize the effects of various perturbing factors (e.g. noise, or contaminants). In particular, focus is required on the automatic discrimination between true and false events. Although a certain degree of discrimination is already achieved by the selection criteria and the set thresholds, a visual inspection of unusual events is still required.

- Wavelength multiplexing. In chapter 3, section 3.2.2, we experimentally determined that, using our current setup, the color camera can discriminate between two particles with $5 \mathrm{~nm}$ difference in radius. Therefore, a certain degree of multiplexing using different particle sizes can be achieved. A solution to increase the multiplexing capabilities is to use various shapes of nanoparticles.

As already mentioned the performance of the sensing platform discussed here does not yet meet that of established platforms, such as SPR. The results obtained in this thesis can be seen as proof of concept rather than as the ultimately achievable sensitivity of the method. As already mentioned, the LOD obtained in the DNA detection, using the 
experimental setup developed and described in this thesis, make us believe that the efficiency of this approach in the protein assay may be substantially improved.

We believe that the overall performance of the system presented in this thesis shows potential in the field of biosensing applications. The wavelength multiplexing and parallelization possibilities as well as the affordable technology (e.g. color camera), without the necessity for a dedicated instrument represent strong points supporting this method. 


\section{References:}

[1] C.-Y. Yang, E. Brooks, Y. Li, P. Denny, C.-M. Ho, F. Qi, W. Shi, L. Wolinsky, B. Wu, D.T.W. Wong, and C.D. Montemagno, "Detection of picomolar levels of interleukin-8 in human saliva by SPR," Lab on a Chip, vol. 5, 2005, pp. 1017-1023.

[2] F. Song, F. Zhou, J. Wang, N. Tao, J. Lin, R.L. Vellanoweth, Y. Morquecho, and J. Wheeler Laidman, "Detection of oligonucleotide hybridization at femtomolar level and sequence-specific gene analysis of the Arabidopsis thaliana leaf extract with an ultrasensitive surface plasmon resonance spectrometer," Nucleic Acids Research, vol. 30, 2002, p. e72.

[3] R. Verdoold, R. Gill, F. Ungureanu, and R.P.H. Kooyman, "Femtomolar DNA detection by parallel colorimetric darkfield microscopy of functionalized gold nanoparticles," Biosensors and Bioelectronics, 2011.

[4] C.L. Baciu, J. Becker, A. Janshoff, and C. Sönnichsen, "Protein-Membrane Interaction Probed by Single Plasmonic Nanoparticles," Nano Letters, vol. 8, May. 2008, pp. 1724-1728.

[5] N. Gupta, "Biosensors Technologies: Acousto-Optic Tunable Filter-Based Hyperspectral and Polarization Imagers for Fluorescence and Spectroscopic Imaging," Methods in Molecular Biology, vol. 503, 2009, pp. 239-258.

[6] B. Lu, J. Xie, C. Lu, C. Wu, and Y. Wei, “Oriented Immobilization of Fab' Fragments on Silica Surfaces," Analytical Chemistry, vol. 67, Jan. 1995, pp. 83-87.

[7] M.M. Harmsen and H.J. Haard, "Properties, production, and applications of camelid single-domain antibody fragments," APPLIED MICROBIOLOGY AND BIOTECHNOLOGY, vol. 77, 2007, pp. 13-22.

[8] J.C. Hulteen and R.P. van Duyne, "Nanosphere lithography: A materials general fabrication process for periodic particle array surfaces," Journal of Vacuum Science \& Technology A: Vacuum, Surfaces, and Films, vol. 13, 1995, pp. 1553-1558. 


\section{Samenvatting}

Dit proefschrift behandelt de ontwikkeling van een nieuwe optische analyse methode voor het detecteren van biomoleculen in lage concentraties en maakt gebruik van gouden nanodeeltjes die in een donkerveld microscoop apart zichtbaar gemaakt kunnen worden. Het aantonen van het proof-of-principle staat centraal, gevolgd door een bepaling van de detectiegrens voor het aantonen van eiwitten, op basis van een specifieke immuunreactie.

Hoofdstuk 1 is een samenvatting van verschillende bioanalyse technieken, waarbij de nadruk ligt op optische methodes.

In hoofdstuk 2 wordt beschreven hoe we gebruik maken van theoretische modellen, waarmee we de optimale eigenschappen van de goud nanodeeltjes kunnen bepalen voor gebruik als biosensor. Eerst hebben we de lokale oppervlakte plasmon resonantie (LSPR) bestudeerd voor goud nanodeeltjes van diverse afmetingen. Door gebruik te maken van numerieke methoden was het mogelijk om de optische eigenschappen van de nanodeeltjes te bepalen, evenals de optimale omstandigheden voor het gebruik als biosensor. Vervolgens ontwierpen we twee strategieën, in de eerste strategie werd de detectielimiet (LOD) bepaald voor een methode zonder label. In de tweede strategie werd wel gebruik gemaakt van signaal versterking door een label. De theoretische LOD voor een molekuul dat een affiniteit constante van $10^{7} \mathrm{M}^{-1}$ heeft met de receptor is geschat op $10 \mathrm{nM}$ zonder label en 10 pM met label. Daarnaast hebben we een nieuw concept uitgewerkt waarbij de responsie van meerdere individuele goud nanodeeltjes parallel wordt gedetecteerd. Deze methode maakt het mogelijk om concentraties in monsters te bepalen veel lager dan de affiniteit constante tussen receptor (antilichaam) en analyte (eiwit). Een uitwerking van deze concepten wordt beschreven in de hoofdstukken 3, 4 en 5 . 
In hoofdstuk 3 introduceren we detectie van eiwitten door de kleurverandering van individuele goud nanodeeltjes te detecteren. In deze methode is elk deeltje een uniek sensor element is. Voor de beeld analyse werd software ontworpen die de vertaling vormt tussen een kleurverandering van een individueel goud nanodeeltje en de interactie van eiwitten (bv. hoeveel eiwitten er gebonden zijn aan een enkel goud deeltje). In experimenten werd het verband gelegd tussen de adsorptie van eiwitten en de kleurverandering. De resultaten hiervan worden ondersteund door de theoretische simulaties. Als toevoeging hierop werd een experiment uitgevoerd voor het bepalen van de werkelijke LOD in een immuno-ractie experiment zonder versterking. Door gebruik te maken van parallelle detectie was het mogelijk om een LOD van $40 \mathrm{nM}$ te meten (geit $\operatorname{IgG})$, hetzelfde als theoretische bepaald in hoofdstuk 2. Helaas was het niet mogelijk om met deze methode de interactie van één enkel eiwit molecuul te meten.

In hoofdstuk 4 tonen we aan dat het meten van de interactie van één enkel eiwit molecuul wel mogelijk is door gebruik te maken van versterking met een goud nanodeeltje. Hiervoor werd ook gebruik gemaakt van parallelle detectie van kleurverandering met behulp van een standaard donker veld microscoop. De resultaten laten zien dat het mogelijk is multiplexing toe te passen om zo specifieke eiwit interacties te meten.

In hoofdstuk $\mathbf{5}$ hebben we gebruik gemaakt van een sandwich strategie. Hiermee konden we het detectie vermogen van de versterkte methode bepalen. Een target eiwit werd specifiek 'gevangen' tussen twee (immuun-)receptoren. Deze techniek wordt veel gebruikt in conventionele eiwit bepalingen zoals ELISA. De resultaten tonen aan dat optimalisatie van deze strategie nodig is. Tot op dit moment werden de gouden nanodeeltjes willekeurig op het oppervlak geplaatst met als gevolg dat er een variatie was in de hoeveelheid deeltjes per beeldvlak. Dit is een van de redenen voor de matige gevoeligheid die we hebben behaald.

In hoofdstuk 6 wordt de huidige detectie methode geëvalueerd en mogelijkheden worden gegeven voor het verbeteren van de methode voor gebruik en onderzoek in de nabije toekomst. 


\section{Acknowledgements}

Finally, the end of a chapter in my life is near. This would not have been possible without the help of all of you. I want to take the time to express my gratitude especially towards:

- my daily supervisor, Rob Kooyman, for giving me the chance to work in this very interesting project.Your support and guidance over all this period was invaluable. Additionally, because of his thoughtful comments and constructive critique made me better understand the difference between a good and a not so good scientist.

- my promoter, Vinod Subramaniam, for his support and guidance throughout all the process of making this thesis. Thank you Vinod for taking the time and energy to help me finishing my thesis.

- Our secretary, Sylvia Winters. She was and still is the lighthouse of all PhD students in the bureaucracy paperwork. Her cheerful attitude and kindness makes your day easier. That is why at Sylvia's office is always a cue.

- My colleagues, Remco\&Ilze, Robert, Dodo, Aufried, Wilma, Eldrich for everything: from all the help during the project's hard time to the chit-chats. Sometimes with you guys work was pure fun. Dodo, you should be a group leader as you have the power to inspire people! At least you made me do things (which normally I would not do) with great joy. Remco, I thank you for all the good times we had: working, discussing, working and playing. It was quite fun working with you and the way I know you, you already have countless friends where you are right now.

- Tom, Yanina, Christian and Srirang for all the nice and challenging discussions. I really enjoyed those times, we should have had more of those.

- My friend Jithin and Shashank. Guys, we (me and Constantin) appreciate all the nice time we had. Too bad that it was too little.

- My NL friends Cristina\&Bart, Corina\&Guido, Dana\&Ronny for all the support during this period. I loved our dinners and all the long discussions on so many topics. Thanks guys, for being there for me. I hope to make it up to you in the near future. 
Maria, Camelia\&Andrei. Dragilor, apai fara voi sincer viata mea ar fi foarte saraca. De cand ati intrat in viata mea m-ati facut sa ma simt ca o sora de-a voastra. Mai tembela dar totusi...nu as putea niciodata sa va multumesc suficient pentru tot ajutorul dat de-a lungul timpului. $\mathrm{Nu}$ exista cuvinte destule sa exprime recunostinta mea fatza de voi.

- Fetele mele de la strumfi si prichindei. De cand v-am gasit acum aproape 2 ani pur si simplu viata a devenit mai frumoasa. Va multumesc ca ati fost alaturi de mine in cele mai grele momente, ca am reusit sa impartim si ganduri frumoase si greutati, sfaturi si ajutor neconditionat. $\mathrm{Cu}$ voi viata mea s-a imbogatit cu 50 de prietene. Va multumesc pentru tot.

- Familiei mele. Mama si tata, va multumesc pentru cum m-ati crescut si as vrea sa puteti sa simtiti recunostinta mea pentru faptul ca m-ati facut OM. Cristi, fratiorul meu cel mare iti multumesc pentru tot. Copilaria noastra a fost frumoasa impreuna si suportul tau de nepretuit. Alex, puiule, iti multumesc pentru tot: toate amintirile frumoase si tot ce va urma. Irina, iti multumesc ca ai grija de zapaceala asta mic. Socrilor mei, Vassa si Gheorghe pentru ca m-au primit in casa lor si m-au facut fiica lor. Cuvintele frumoase si povetele ne-au ajutat pe parcursul acestui drum lung. Iancu si Dani va multumim ca, desi suntem departe, voi tot aveti timp sa va ganditi la noi.

- Cosmin, tu esti jumatatea mea. Tu esti cel caruia ii pot spune toate ofurile mele, toate bucuriile, cu tine impart si painea cea dulce si mierea cea amara. Impreuna am fost si la bine si la greu. Si nu m-ai lasat sa cad niciodata jos. Ci m-ai ridicat si ai mers tu in locul meu. Sper sa fiu vreodata demna sa iti rasplatesc sacrificiile pe care le-ai facut.

- Puilor mei Ana \& Gabriel. Voi sunteti lumina ochilor mei: de cand ati venit pe lume ati dat sens vietii mele facandu-ma mama. Va multumesc ca imi inseninati ziua cu rasetele voastre cristaline, cu prostioarele voastre zilnice si mai ales ca ma rasplatiti in fiecare zi cu o moneda de nepretuit: dragostea. Va multumesc puilor si sper sa am puterea si priceperea sa va pot face si pe voi OAMENI.

- Iti multumesc tie Doamne ca mi-ai dat puterea sa razbesc in zilele mele negre si ca ai fost alaturi de mine cand simteam ca lumea se darama. Iti multumesc ca mi-ai dat speranta si ca pot sa merg mai departe stiindu-TE langa mine. 


\section{List of publications}

\section{Journal articles (peer reviewed)}

R. Verdoold, Ron Gill, F. Ungureanu, R. Molenaar, R. Kooyman "Femtomolar DNA detection by parallel colorimetric darkfield microscopy of functionalised gold nanoparticles", Biosensors and Bioelectronics, accepted for publication

Constantin Ungureanu, Rene Kroes, Wilma Petersen, Tom A. M. Groothuis, Felicia Ungureanu, Hans Janssen, Fijs W. B. van Leeuwen, Rob P. H. Kooyman, Srirang Manohar and Ton G. van Leeuwen, "Light interactions with gold nanorods and cells: implications for photothermal nanotherapeutics", Nanoletters, 2011, 11 (5), pP $1887-1894$

F. Ungureanu, Dorothee Wasserberg, Ni Yang, Remco Verdoold, Rob Kooyman Immunosensing by colourimetric darkfield microscopy of individual gold nanoparticle- conjugates, 2010, Sensors and Actuators B: Chemical,150, 2, 529536

F. Ungureanu, L. Voicu, I. R. Andrei, The modification of phenyl-pyridine compounds under UV Irradiation: FTIR investigation, 2006, J. Optoel. and Adv. Mat., 8, 1, 315-318

\section{Proceedings articles}

Ungureanu, F., Halamek, J., Verdoold, R., Kooyman, R.P.H., "The use of a colour camera for quantitative detection of protein binding nanoparticles", Progress in Biomedical Optics and Imaging - Proceedings of SPIE 7192, art. no. 719200 .

R. Verdoold, F. Ungureanu, D. Wasserberg and R. P. H. Kooyman, "Gold nanoparticle assays: towards single molecule unamplified DNA detection", Advanced Environmental, Chemical, and Biological Sensing Technologies VI, Proc. of SPIE Vol. 731273120 N-1

R. A. Pascu, L. Voicu, F. Ungureanu, D. Gazdaru, J. Barbe, In vitro studies of the effects of Alkylphenylpyridinium compounds exposed to optical radiation, Communication at the $20^{\text {th }}$ International Congress on Laser Medicine Laser Florence 2005, 10-12 November 
Staicu A., L. Voicu , F. Ungureanu, M. L. Pascu, J. Barbe, R. A. Pascu, About Spectral Studies ofsome Alkylphenylpyridinium Compounds under UV Irradiation, Invited paper at the European COST Action B16 Symposium on Multidrug resistence reversal by inbibition of Transmembrane Transport, Belek-Antalya, Turkey, May 13 th-14th

C. Ungureanu, F. Corniencu, "Person identification using fractal analysis of retina images", Proceedings SPIE 5581, 2004, pp 721-727

\section{Conference contributions (oral presentations):}

R. Verdoold, F. Ungureanu, D. Wasserberg, R. Molenaar, J. Halamek, R.P.H. Kooyman, “Gold nanoparticle assays for single molecule DNA and protein detection", Micro and Nano conference 2008, Ede, the Netherlands, November 182008.

F. Ungureanu, J. Halamek, R. Verdoold, and R.P.H. Kooyman, "The use of a colour camera for quantitative detection of protein-binding nanoparticles," SPIE conference, Photonix 2009, San Fransisco (CA), United States of America, April 142009

R. Verdoold, F. Ungureanu, D. Wasserberg and R.P.H. Kooyman, Gold nanoparticle assays for single molecule detection of unamplified DNA, SPIE conference, Security, Defense and Sensing 2009, Orlando (FL), United States of America, April 142009.

R. Verdoold, F. Ungureanu, D. Wasserberg, R. Molenaar and R.P.H. Kooyman, "Gold nanoparticle assays: Towards single binding event detection", BioNano Europe conference, Münster, Germany, June 152010

\section{Conference contributions (Poster presentations)}

F Ungureanu, R Verdoold, R Molenaar, D Wasserberg, RPH Kooyman,” Gold nanoparticle assays for sensitive detection of biological molecules using colorimetric DF microscopy",MicroNanoNed Conference, Enschede, Netherlands, 16-17 November 2010

R. Verdoold, F. Ungureanu, D Wasserberg, R.P.H. Kooyman, "Gold nanoparticle assays: Towards single binding event detection”, Mesa+ day, Enschede, Netherlands, September 2010 
F Ungureanu, D Wasserberg, R Verdoold, RPH Kooyman," Colorimetric darkfield microspectroscopy of gold nanoparticles as sensing platforms", SPIE conference, Optics+ Photonics, San Diego, USA, 1-5 August 2010

R. Verdoold, F. Ungureanu, D Wasserberg, R.P.H. Kooyman, "Gold nanoparticle assays: Towards single binding event detection", NanoBio Europe Conference, Munster, Germany, June 2010

F Ungureanu, D Wasserberg, R Verdoold, RPH Kooyman," Colorimetric darkfield microspectroscopy of gold nanoparticles as sensing platforms", 1st biosensing conference (Elsevier), Bristol, United Kingdom, November 10 13

R. Verdoold, D. Wasserberg, F. Ungureanu, T. Brinkman, E. Tromp and Rob Kooyman, "Gold nanoparticle assays: towards sensitive detection of nonamplified $D N A^{\prime \prime} 1$ st biosensing conference (Elsevier), Bristol, United Kingdom, November $10-13$.

R. Verdoold, F. Ungureanu, D. Wasserberg, T. Brinkman, R. Molenaar and R.P.H. Kooyman "Functionalised Gold Nanoparticles - towards sensitive detection of biomolecules", Micro Nano conference, Enschede, the Netherlands, November 5 -6.

R. Verdoold, F. Ungureanu, D. Wasserberg, T. Brinkman, R. Molenaar and R.P.H. Kooyman "Functionalised Gold Nanoparticles - towards sensitive detection of biomolecules", Annual MESA+ meeting, Enschede, the Netherlands, September 21. 